\title{
Three essays in choice and social choice theory
}

Citation for published version (APA):

Protopapas, P. C. (2019). Three essays in choice and social choice theory. [Doctoral Thesis, Maastricht University]. ProefschriftMaken Maastricht. https://doi.org/10.26481/dis.20190118pp

Document status and date:

Published: 01/01/2019

DOI:

10.26481/dis.20190118pp

Document Version:

Publisher's PDF, also known as Version of record

\section{Please check the document version of this publication:}

- A submitted manuscript is the version of the article upon submission and before peer-review. There can be important differences between the submitted version and the official published version of record.

People interested in the research are advised to contact the author for the final version of the publication, or visit the DOI to the publisher's website.

- The final author version and the galley proof are versions of the publication after peer review.

- The final published version features the final layout of the paper including the volume, issue and page numbers.

Link to publication

\footnotetext{
General rights rights.

- You may freely distribute the URL identifying the publication in the public portal. please follow below link for the End User Agreement:

www.umlib.nl/taverne-license

Take down policy

If you believe that this document breaches copyright please contact us at:

repository@maastrichtuniversity.nl

providing details and we will investigate your claim.
}

Copyright and moral rights for the publications made accessible in the public portal are retained by the authors and/or other copyright owners and it is a condition of accessing publications that users recognise and abide by the legal requirements associated with these

- Users may download and print one copy of any publication from the public portal for the purpose of private study or research.

- You may not further distribute the material or use it for any profit-making activity or commercial gain

If the publication is distributed under the terms of Article $25 \mathrm{fa}$ of the Dutch Copyright Act, indicated by the "Taverne" license above, 


\section{Three Essays in Choice and Social Choice Theory}


(C) copyright Panos Protopapas, Maastricht 2019

Printing: ProefschriftMaken || www.proefschriftmaken.nl

ISBN 9789463802086

All rights reserved. No part of this publication may be reproduced, stored in a retrieval system or transmitted, in any form or by any means, electronic, mechanical, photocopying, recording or otherwise, without prior permission of the author or the copyright-owning journals for previous published chapters. 


\title{
Three Essays in Choice and Social Choice Theory
}

\section{DISSERTATION}

\author{
to obtain the degree of Doctor at the Maastricht University, \\ on the authority of the Rector Magnificus, \\ Prof.dr. Rianne M. Letschert \\ in accordance with the decision of the Board of Deans, \\ to be defended in public \\ on Friday 18 January 2019, at 10:00 hours
}

by

Panagiotis Constantine Protopapas 

Supervisor(s):

Prof.dr. B.E. Klaus, University of Lausanne, Switzerland Prof.dr H.J.M. Peters

\section{Assessment Committee:}

Prof.dr. A.J. Vermeulen (chair)

Dr. F. Klijn, Institute for Economic Analysis, Barcelona, Spain Prof.dr. J. Massó, Autonomous University of Barcelona, Spain Prof.dr. L.P. Santos Pinto, University of Lausanne, Switzerland 



\section{Acknowledgments}

First, I would like to thank my supervisor, Bettina Klaus; without her wisdom, support, coffee and biscuits, and patience throughout the years, this thesis would have never materialized. I would also like to thank her for the chance she gave me to present my work in numerous conferences and workshops across Europe and the USA.

Second, I would like to thank my co-supervisor in Maastricht, Hans Peters, for all his help and guidance he provided me with -especially towards the end of my $\mathrm{PhD}$, as well as for all the lovely lengthy discussions we had in his office.

Third, I am grateful to the thesis committee members, Flip Klijn, Jordi Massó, Luis Pedro Santos-Pinto, and Dries Vermeulen for their very constructive and valuable feedback which helped me improve the quality of my thesis.

Fourth, I want to thank my wife Electra for all her emotional support during the last five years. Without her I would never be able to thank my son Aris for being unaware of how huge a motivation he is for me (even if he uses this thesis as a coloring book in the near future).

Fifth, I would like to thank my parents, Costas and Maria, as well as my brother Sotiris, for their support throughout the years. The education, motivation, and attitude necessary for a $\mathrm{PhD}$ would not be in place without them.

Finally, I would like to thank Alex, Giannis, Jay, Kostas, Manolis, Manos, Michalis, Mitsos, Petros, and Vaggelis for their friendship and support throughout the years. 



\section{Contents}

Introduction

1 Set and revealed preference axioms for multi-valued choice 1

1.1 Introduction . . . . . . . . . . . . . . . . . . . . . . . . 1

1.1.1 Background .................... . . 1

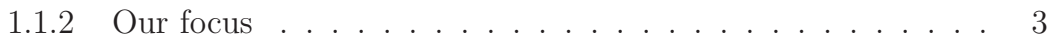

1.2 Model and properties . . . . . . . . . . . . . . . . 4

1.2.1 Model and basic definitions . . . . . . . . . . . . 4

1.2.2 Weak axiom of revealed preference . . . . . . . . . . 5

1.2.3 Weak independence of irrelevant alternatives . . . . . . . . 7

1.2.4 Independence of irrelevant alternatives . . . . . . . . . . 10

1.3 Weak and strong sets . . . . . . . . . . . . . . . . . . . 13

1.3 .1 Weak sets . . . . . . . . . . . . . . . . 13

1.3 .2 Strong sets . . . . . . . . . . . . . . . . . . . . 14

1.4 Partitions induced by WIIA and IIA . . . . . . . . . . . . . . . 15

1.4.1 Weak sets and WIIA . . . . . . . . . . . 15

1.4 .2 Strong sets and IIA . . . . . . . . . . . . . . . . . . 17

1.5 Summary . . . . . . . . . . . . . . . . . . . 19

2 Solidarity for public goods under single-peaked preferences: $\begin{array}{ll}\text { Characterizing target set correspondences } & 21\end{array}$

2.1 Introduction . . . . . . . . . . . . . . . . . . . . . . . . . 21

2.2 The model . . . . . . . . . . . . . . . . . . . . . . . 26

2.3 Choice correspondences . . . . . . . . . . . . . . . . . . . . . . 32

2.4 Properties of choice correspondences . . . . . . . . . . . . . . . 34

2.5 Characterizing target set correspondences . . . . . . . . . . . . . . . 41

2.A Proofs of Proposition 2.1 and Corollary 2.2 . . . . . . . . . . . . . 44

2.B Proof of Lemma 2.2 . . . . . . . . . . . . . . . . . . . . . . 47

2.C Proof of Proposition $2.5 \ldots \ldots \ldots \ldots$. . . . . . . . . . . . 51 
2.D Proof of Theorem 2.1 . . . . . . . . . . . . . . 55

3 On strategy-proofness and single-peakedness: median-voting over $\begin{array}{ll}\text { intervals } & 67\end{array}$

3.1 Introduction . . . . . . . . . . . . . . . . . . 67

3.2 The model . . . . . . . . . . . . . . . . . . . . . 70

3.3 Choice functions and correspondences . . . . . . . . . . . . . . 73

3.3.1 Generalized median rules and correspondences . . . . . . . . 73

3.3.2 Median rules and correspondences . . . . . . . . . . . . . 77

3.4 Properties of correspondences . . . . . . . . . . . . . . . 79

3.5 Results . . . . . . . . . . . . . . . . . . . . 81

3.5.1 Interrelations between properties . . . . . . . . . . . . 81

3.5.2 Results in the single-peaked domain $\mathcal{R}$. . . . . . . . . . . 82

3.5.3 Results in the single-peaked and symmetric domain $\mathcal{S}$. . . . 86

3.5.4 Independence of properties . . . . . . . . . . . . . . . . 87

3.5.5 Discussion . . . . . . . . . . . . . . . . . . . . . . . 88

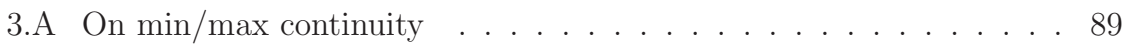

3.B Proofs of Propositions 3.2 and $3.3 \ldots \ldots$. . . . . . . . . . 90

3.C Proofs of Propositions 3.4, 3.5, and 3.6 . . . . . . . . . . . 95

3.D Proof of Theorem 3.1 (equivalence of statements (ii) and (iii)) . . . . . 103

$\begin{array}{lr}\text { Bibliography } & 109\end{array}$ 


\section{Introduction}

Many situations in life require a decision to be made in order to resolve some issue that has arisen. Such situations are essentially cases where the decision must be chosen from a set of possible alternatives. For example, having to choose on a rainy day whether to take an umbrella, an anorak, or perhaps neither; or having to choose what to order at a restaurant, just the main course or the three course meal? How about a drink as well? Of course, the choice outcome in any of these issues depends on the preferences of the individual that this choice concerns. Some people do not mind getting a little wet if it rains, so perhaps they might opt to take neither an umbrella nor an anorak with them on a rainy day. Some other -very weird- people never have dessert, so for them choosing a three course meal is out of the question.

However, not all decision choices are made by just one person; some issues concern a group of people. In this case, a suitable "mechanism" must be used that will take into account the preferences of all members in the group before reaching a decision. For example, many countries use plurality voting when electing a new government. This mechanism chooses the party that received the highest number of votes, or in other words, the most preferred party according to the preferences of the plurality. As a second example, consider a book club where members of the club take turns every week in suggesting a book for everyone in the club to read. This kind of mechanism, where the decision is made by just one member of the group, but the decision maker alternates every week, is called serial dictatorship.

In this thesis, both cases where the decision is taken either by a single person or by a group of people are considered. In the first chapter, we study cases where the issue concerns a single person, henceforth, agent. Specifically, we are interested in whether the agent's preferences, according to the choices he makes, should be considered "rationalizable". For a brief (and not completely accurate) explanation of what is meant by the term rationalizability of preferences, consider the following example. An agent whose preferences are rationalizable has to decide what to order from a restaurant's menu. The available choices are hamburger, salad, beer, 
and coffee. The agent decides to order salad and beer; therefore, we can deduce that he prefers this combination over every other available combination on the menu (e.g., hamburger and beer or salad and coffee). Nevertheless, just before placing his order, the waiter informs him of the daily special, steak, which is not listed in the menu. Perhaps now the agent will change his order to steak and beer or steak and coffee; after all, neither combination was previously available, and therefore, such a change would be rational. However, changing his order to hamburger and beer would not be rational; the combination of hamburger and beer was also available initially, when the agent chose to order salad and beer, and we deduced that according to his rationalizable preferences, he prefers salad and beer over hamburger and beer.

In the second and third chapters, we study cases where the issue concerns a group of people, henceforth, agents, and a decision mechanism must be implemented in order to consider the preferences of all agents in the group before reaching a decision. Here, we are interested to find out how this mechanism might function when certain requirements, henceforth, properties, must be met. For example, perhaps it would be useful that all agents declare their true preferences instead of trying to manipulate (by lying) the decision chosen by the mechanism. Due to this, we could require that the mechanism does not "reward" liars by making sure that an agent would always get the same result or a more favorable one when telling the truth. Another property of the mechanism could be that all agents in the group are treated equally, irrespective of gender, race, social standing, or religious beliefs. Furthermore, in an environment with often-changing circumstances, a mechanism that would mitigate any positive or negative changes "fairly" among the group of agents would perhaps be welcome; therefore, the following property would perhaps be in order. Following a positive (negative) change in the circumstances, all agents get better off (worse off) compared to their initial situation. However, it is necessary to bear in mind that not all combinations of properties are always "compatible". One of the first to point out this incompatibility was Kenneth J. Arrow with his famous impossibility theorem that states the following. In every voting situation with three or more candidates, there exists no electoral system that can be efficient, ${ }^{1}$ independent over irrelevant alternatives

\footnotetext{
${ }^{1}$ In a typical voting situation, efficiency essentially requires that the elected candidate was voted for by at least one voter.
} 
(IIA) ${ }^{2}$ and be non-dictatorial ${ }^{3}$ as well (Arrow, 1950).

We proceed by providing a more in-depth summary of each chapter.

\section{Chapter 1: Set and revealed preference axioms for multi-valued choice}

The first chapter was written in collaboration with Hans Peters. It is chronologically the last chapter of this thesis, but is presented first due to its more general nature of the topic in choice theory, relative to the other two chapters. The main part of the work in it took place during the six months I spent in Maastricht, spread over the last three years.

In this chapter, we consider situations where an issue has arisen with a number of alternative ways to deal with it, and some individual needs to choose one, or more, of these alternatives in order to deal with this situation. In the sequel, we refer to all the possible ways to deal with the issue by the term choice set and to the individual by the term choice correspondence; that is, given a choice set of alternatives, a choice correspondence will choose a subset of this choice set, the chosen set.

Specifically, we are interested in choice correspondences satisfying two properties. First, the property of independence of irrelevant alternatives (IIA), as well as a weaker version of it, and second, the weak axiom of revealed preference for sets (WARP) $)^{4}$

We show the existence of connections between these properties and two particular collections of choice sets, called weak and strong sets, which in turn partially or completely determine the choice correspondences satisfying the above independence properties. In other words, if we were to refer to the restaurant example on page iii, we find that by knowing what an agent will order at some restaurants, specifically the ones whose menus are included in weak and strong sets, we are able to determine what this agent will order at every restaurant, assuming the way he makes his choices is according to the two aforementioned conditions.

\footnotetext{
${ }^{2}$ Loosely speaking, IIA requires that the removal of non-winning candidates from an election should not affect the result. For example, if in an election between candidates $a, b$, and $c, a$ is the winner, then IIA requires that in an election between just candidates $a$ and $b, a$ to be the winner as well, since the removal of non-winning candidate $c$ is irrelevant.

${ }^{3}$ If in every voting situation a specific voter can dictate the result, then we say that this voter is a dictator. An electoral system is non-dictatorial if it does not allow the existence of dictators.

${ }^{4}$ Consider again the restaurant example on page iii. Since salad and beer are chosen initially, the agent reveals his preference for salad and beer over any other available combination in the menu (e.g., salad and coffee). WARP requires that at some other choice set, where salad an beer are available, the agent will not select salad and coffee, and thus reveal that he prefers salad and coffee over salad and beer.
} 


\section{Chapter 2: Solidarity for public goods under single-peaked preferences: Characterizing target set correspondences}

The second chapter was written in collaboration with Bettina Klaus and is chronologically the first chapter of this thesis. It has been presented in conferences at Istanbul's Bilgi University, Budapest's Corvinus University, Tbilisi State University, Maastricht University, the University of Zurich, Lund University, the University of Southern Denmark, and the University of Lausanne. Moreover, a poster of it has been presented at Grenoble Alps University.

Here, we consider the problem that a city planner might face when having to choose a group of locations where an identical public good will be provided to the citizens of the city in question. For example, choosing the locations of public parking positions, or in other words, choosing the location of a public parking zone. In addition, the city planner's choice needs to take into account the preferences of the citizens, who are the ones to use this parking zone. Loosely speaking, said preferences are single-peaked. ${ }^{5}$

Similarly to the first chapter, the city planner making the choice will be referred to as a choice correspondence and we will be interested that this choice correspondence satisfies certain properties. However, in contrast with the previous chapter, these properties are now related with the citizens' preferences and moreover, two of them require a notion of solidarity among the citizens. Namely, the three properties are efficiency, ${ }^{6}$ population-monotonicity, ${ }^{7}$ and (a version of) replacement-dominance, ${ }^{8}$ with the two latter ones requiring the aforementioned notion of solidarity.

We show that if efficiency and either population-monotonicity or replacementdominance are to be satisfied by a choice correspondence, then this choice correspondence belongs to a particular class of correspondences, that of target set correspondences. Loosely speaking, this result implies the following. If the city planner's choice has to satisfy these properties, then he should first choose alone

\footnotetext{
${ }^{5}$ Single peakedness of preferences implies the following. Each citizen has a single most favorite location (his peak) when parking his car, for example, right outside his home; moreover, if this location is taken, then he would prefer to park as closely as possible to his most favorite location.

${ }^{6}$ When a parking zone is chosen, efficiency guarantees that no other parking zone could have been chosen instead and would have made some citizens better off, and no citizen worse off.

${ }^{7}$ If following the addition of some new citizens, a parking zone change is in order, then all citizens, excluding the newcomers, must be affected in the same way; either nobody gets worse off, or nobody gets better off.

${ }^{8}$ If following a citizen's change of preferences (e.g., after moving to a new home), a parking zone change is in order, then all other citizens must be affected in the same way; either nobody gets worse off, or nobody gets better off.
} 
where the parkinoneg zone should be, then ask the citizens about their preferences, and finally, only change his initial choice if all citizens unanimously agree with this change.

\section{Chapter 3: On strategy-proofness and single-peakedness: median- voting over intervals}

The third chapter was single-authored and was chronologically the second chapter of this thesis; it was written under the close supervision and guidance of Bettina Klaus. It has been presented in conferences at the University of Lausanne, the University of Zurich, the University of York, Paris Dauphine University, and the University of Innsbruck.

The problem considered here is in some ways similar to the one considered in the previous chapter. Specifically, the problem is the following. A city planner has to choose a set of (tax) policies which will affect all citizens/voters, with the possible choices spanning from extreme left-wing choice to extreme right-wing choices. For example, the decision to increase the tax rate of high earners and decrease that of low earners would be a left-wing policy, while on the other hand, abolishing health-related taxes all together and having citizens pay for their health insurance through the private sector would be a right-wing policy. All voters have their own preferences about policy choices, which they announce to the city planner by voting. These preferences are again "based on" single-peaked preferences; for example, if a voter's most favorite policy (peak) is a left-wing one, then he also prefers policies closer to his peak over policies further away. Therefore, he would also prefer central policies over right-wing policies.

Similarly to the previous chapter, we will refer to the city planner using the term choice correspondence and will be interested that this choice correspondence satisfies certain properties, all of which related with the citizens' announced preferences, i.e., the citizens' votes. To be more precise, the main three properties we are interested in are strategy-proofness, ${ }^{9}$ peaks-onliness, ${ }^{10}$ and anonymity. ${ }^{11}$

Our two main results are as follows. First, if strategy-proofness and peaksonliness are to be satisfied by a choice correspondence, then this choice correspondence belongs to a particular class of correspondences, that of generalized median correspondences. Second, if anonymity is also to be satisfied, then this choice cor-

\footnotetext{
${ }^{9}$ When a voter lies and does not announce his true preferences, the result cannot improve in his favor. In other words, a voter cannot strategize in order to improve the result in his favor.

${ }^{10}$ All voters are only allowed to declare, by voting, their most favorite policy. They cannot also declare their second most favorite, third most favorite, etc.

${ }^{11}$ All voters have equal rights in the decision, in other words, all voters get one vote each.
} 
respondence belongs to a sub-class of generalized median correspondences, called the class of median correspondences. Loosely speaking, the second result implies the following. ${ }^{12}$ If during an election, the city planner's choice has to satisfy these properties, then he should first stuff the ballot box with a number of votes of his choice, then ask the citizens to cast their votes, and finally after combining all stuffed and cast votes, choose the policy favored by the median vote (in a sense, the central vote).

At first, this seems as an unwanted situation giving the voters no say in the outcome; however, this is not entirely true. Although a high number of stuffed votes implies that indeed, voters cannot influence the outcome, a low number of stuffed votes achieves a different effect. Specifically, depending on how "low" this number is, the voters might have complete control on the outcome, in the case of zero stuffed votes, or have some degree of control on the outcome, with this degree depending on the number of stuffed votes. ${ }^{13}$

\footnotetext{
${ }^{12}$ The first result, which does not include anonymity, is a little more general and due to this, difficult to explain concisely in an informal way.

${ }^{13}$ For an example where voters only have some degree of control over the outcome, consider the following. In many countries, a supermajority of the parliament is required to agree with proposed constitutional amendments, in order for these to take place. In such cases, the stuffed votes could be though of as favoring the status-quo, i.e., no change in the constitution.
} 


\title{
Chapter 1 \\ Set and revealed preference axioms for multi-valued choice
}

\begin{abstract}
We consider choice correspondences for arbitrary sets of alternatives, and focus on the condition of independence of irrelevant alternatives and on a weaker version of it, as well as on the weak axiom of revealed preference for sets. We establish connections between these conditions and their relations with collections of choice sets, called weak and strong sets, that partially or completely determine the choice correspondences satisfying the above independence properties.
\end{abstract}

\section{$1.1 \quad$ Introduction}

\subsubsection{Background}

This chapter contributes to a question with a long history. For single-valued choice functions the condition of Independence of Irrelevant Alternatives (IIA) requires that if the alternative chosen from a choice set is still available in a subset, then it should be chosen in that subset. This condition already occurs in Nash (1950) as condition no. 7 in Nash's axiomatic bargaining model. For collections of choice sets that are closed under a nonempty intersection - as is the case in the present paper - and (single-valued) choice functions IIA is equivalent to the Weak Axiom of Revealed Preference; the latter requiring the revealed preference relation to admit no cycles of length two. The question is how to extend the IIA condition to (multi-valued) choice correspondences.

The probably most obvious extension was also proposed by Nash in an informal note in 1950 - see Shubik (1982, p. 420): if $F$ is the choice correspondence choosing a subset of every choice set, $X$ is a choice set, and $F(X)$ has a nonempty intersection with $Y$, a subset of $X$, then $F(Y)$ should be equal to this intersection. 
This condition also appears as Postulate 6 in Chernoff (1954) and Condition C4 in Arrow (1959). The most common and obvious interpretation is that the set of alternatives chosen by a choice correspondence should be viewed as the set of best alternatives (in some sense or another) among the available ones: that is, each of these alternatives is also best in any subset of available alternatives to which it belongs.

A second possible extension would say that in such a situation, $F(Y)$ should at least contain the intersection of $F(X)$ and $Y$. In terms of the interpretation above, it could be that additional best alternatives become available in the smaller set. For instance, the first preferred choice of wine from a restaurant's menu is no longer available, making the second preferred choice a best alternative (additional to the still available best menu choices). This condition was first proposed as Postulate 4 in Chernoff (1954), and has consequently been referred to as the Chernoff property (e.g., Moulin, 1985, 1988). It appears as Property $\alpha$ in Sen (1971).

In a similar vein, a third extension is to require that the intersection of $F(X)$ and $Y$ should contain $F(Y)$, hence $F(Y)$ is a subset of $F(X)$. In other words, $F$ still chooses among the best elements, but not necessarily all available ones. Think of choosing a committee within a society: for a subset of the society one may need to choose a strictly smaller committee, even if more members of the original committee are still available. Or, in terms of the restaurant's menu choice, the lunch menu may be a subset of the dinner menu, but also lunch itself may be lighter than dinner: one may want to consume wine of just one brand instead of several, even if more brands are still available. This extension appears as condition W2 in Schwartz (1976).

In this chapter the focus is on the first and third extensions, to be called IIA and Weak IIA (WIIA). A still weaker version of the latter condition is the following (e.g., Fishburn, 1973) if $F(X)$ is contained in $Y$, then $F(Y)$ should be contained in $F(X)$. This condition, studied in Aizerman and Malishevski (1981), is referred to as the Aizerman condition; it is implied by Condition W3 in Schwartz (1976).

Following a tradition initiated for consumer theory by Samuelson (1938) and Houthakker (1950), and continued for general choice problems by - among others - Arrow (1959) and Richter (1966), most of the literature focuses on rationalizability: when does a choice correspondence always pick the set composed by those alternatives that are maximal for some binary relation on the set of alternatives? For instance, Arrow (1959) shows that a choice correspondence is rationalizable by a complete and transitive binary relation if and only if it satisfies IIA. Sen (1971) shows that a choice correspondence is rationalizable by a binary relation if 
and only if it satisfies the Chernoff condition and a condition proposed as Property $\gamma^{1}$ but later also referred to as the Expansion condition (e.g., Moulin, 1985). Adding to this the condition of Aizerman results in the choice correspondence being rationalizable by an ordering which is complete and has a transitive strict part (Schwartz, 1976; Moulin, 1988). Finally, Aizerman and Malishevski (1981) show that a choice correspondence satisfies both the Chernoff and the Aizerman condition if and only if it is pseudo-rationalizable by a collection of single-valued, complete, and transitive orderings; that is, if in each choice set, the choice correspondence picks the maximal elements of all the orderings in this collection.

\subsubsection{Our focus}

As mentioned, we focus on IIA as initiated by Nash (Shubik, 1982) and considered by Arrow (1959), as well as on its weaker version, WIIA, appearing as condition W2 in Schwartz (1976). We assume no structure on the set of alternatives - it can be any finite or infinite set, and we study IIA and WIIA choice correspondences with respect to two closely related questions.

The first question is that of rationalizability. This concerns the existence of a binary relation on the collection of choice sets, thus, sets of alternatives rather than only single alternatives, which rationalizes a given choice correspondence. The usual approach in the literature is to consider revealed preference relations on alternatives instead of sets, with the exception of Brand and Harrenstein (2011). Specifically, they consider set-versions of Chernoff's and Expansion conditions ${ }^{2}$ and obtain a characterization (their Theorem 2) of 'set-rationalizable' choice correspondences. Their set-rationalizability condition is what we call WARP (Weak Axiom of Revealed Preference). We characterize WARP by a different condition and show that this condition is indeed equivalent to the condition of Brand and Harrenstein (2011) - see Lemmas 1.1 and 1.2. We further show that WARP is implied by WIIA if the choice correspondence $F$ is a projection, i.e., $F \circ F=F-$ the latter is implied by WARP, as also observed in Brandt and Harrenstein (2011). In Theorem 1.1 we characterize IIA by WARP combined with another axiom on the revealed preference relation ('Preference Axiom', PA); this is in contrast to the single-valued choice case, where IIA and WARP are equivalent. Finally, we show that IIA implies that the revealed preference relation on choice sets is transitive

\footnotetext{
${ }^{1}$ Property $\gamma$ : for all choice sets $X$ and $Y$, the intersection of $F(X)$ and $F(Y)$ is contained in $F(X \cup Y)$.

${ }^{2}$ Note that Brand and Harrenstein (2011) use the nomenclature of Sen (1971), i.e., $\alpha$ - and $\gamma$-properties instead of Chernoff's and Expansion conditions respectively.
} 
and therefore, in view of WARP, acyclic.

The second question is that of identifying collections of choice sets that, as much as possible, determine the choice correspondence satisfying WIIA or IIA. Given a choice correspondence $F$, a choice set $S$ is called a 'weak set' if, for every choice set $X$, it contains $F(X)$ whenever $F(X)$ has a nonempty intersection with it, and is equal to $F(X)$ whenever $S \subseteq X$. Our main result here is that if $F$ satisfies WIIA and is a projection, then these weak sets partition the sets of alternatives and the restriction of the revealed preference relation to the weak sets is complete and acyclic (Theorem 1.3). A choice set $S$ is called a 'strong set' if $F(X)$ is equal to the intersection of $X$ and $S$ whenever $F(X)$ has a nonempty intersection with $S$. Here, we show that if $F$ satisfies IIA, then these strong sets partition the sets of alternatives and the restriction of the revealed preference relation to the strong sets is complete and acyclic (Theorem 1.4). In this case, the strong sets determine a unique IIA choice correspondence.

In Section 1.2, we introduce the model and the three main conditions on a choice correspondence that we consider (WARP, WIIA, and IIA), establishing relations or lack thereof between them. Section 1.3 introduces the collections of weak sets and strong sets, and establishes some properties of these collections. In Section 1.4 we derive Theorems 1.3 and 1.4 mentioned above. Section 1.5 concludes with a summary of the results of the paper.

\section{$1.2 \quad$ Model and properties}

\subsubsection{Model and basic definitions}

Let $A$ be a finite or infinite set of alternatives and let $\mathcal{A}$ denote the class of its nonempty subsets, i.e., $\mathcal{A}=2^{A} \backslash\{\emptyset\}$. A choice correspondence is a map $F: \mathcal{A} \rightarrow \mathcal{A}$ such that $F(X) \subseteq X$ for every $X \in \mathcal{A}$. A choice correspondence $F$ induces an irreflexive binary relation $R_{F} \subseteq \mathcal{A} \times \mathcal{A}$ by

$$
(X, Y) \in R_{F} \Leftrightarrow \text { there exist } Z \in \mathcal{A} \text { with } X=F(Z) \text { and } Y \subseteq Z
$$

for all distinct $X, Y \in \mathcal{A}$. In this case we say that $X$ is revealed preferred to $Y$ by $F$ and call $R_{F}$ the revealed preference relation of $F$.

Later on we also use the following definitions and notations. A binary relation $R$ on a set $\Omega$ is transitive if $\left(\omega^{1}, \omega^{2}\right),\left(\omega^{2}, \omega^{3}\right) \in R$ implies $\left(\omega^{1}, \omega^{3}\right) \in R$ for all distinct $\omega^{1}, \omega^{2}, \omega^{3} \in \Omega$. The binary relation $R$ has a cycle of length $n$, where 
$n \in \mathbb{N} \backslash\{1\}$, if there are $\omega^{1}, \ldots, \omega^{n} \in \Omega$ such that $\left(\omega^{i}, \omega^{i+1}\right) \in R$ for all $i \in$ $\{1, \ldots, n-1\}$ and $\left(\omega^{n}, \omega^{1}\right) \in R ; R$ is acyclic if it has no cycles of any length.

For a choice correspondence $F$, we use the notation $F^{n}(X)$ as shorthand for $F \circ(F \circ(\ldots(F(X))))$, that is, the $n$-fold composition of $F$ with itself.

In the sequel, we denote a generic choice correspondence by $F$ and consequently, its revealed preference relation by $R_{F}$.

\subsubsection{Weak axiom of revealed preference}

The following definition is the standard notion of revealed preference adapted to our model.

Weak axiom of revealed preference (WARP). For all $X, Y \in \mathcal{A}$, if $(X, Y) \in$ $R_{F}$, then $(Y, X) \notin R_{F}$.

In conformity with the literature, in the revealed preference relation, WARP excludes cycles of length two but not longer cycles (among others, Rose, 1958; Peters and Wakker, 1994; Bossert and Peters, 2009). For completeness, we provide the following example, which contains a cycle of length three, but cycles of arbitrary length can be easily constructed in similar examples.

Example 1.1. Let $A=\{a, b, c\}$ and define $F$ by

$$
F(X)= \begin{cases}\{a, b, c\} & \text { if } X=\{a, b, c\} \\ \{a\} & \text { if } X \in\{\{a, b\},\{a\}\} \\ \{b\} & \text { if } X \in\{\{b, c\},\{b\}\} \\ \{c\} & \text { if } X \in\{\{a, c\},\{c\}\} .\end{cases}
$$

Since $F(a, b, c)=\{a, b, c\}$, it follows that for all $X \subsetneq\{a, b, c\},(\{a, b, c\}, X) \in$ $R_{F}$. Moreover, $F(a, b)=F(a)=\{a\}$ implies that $(\{a\},\{a, b\}),(\{a\},\{b\}) \in$ $R_{F}, F(b, c)=F(b)=\{b\}$ implies that $(\{b\},\{b, c\}),(\{b\},\{c\}) \in R_{F}$, and finally $F(a, c)=F(c)=\{c\}$ implies that $(\{c\},\{a, c\}),(\{c\},\{a\}) \in R_{F}$. Therefore, $F$ satisfies WARP but $R_{F}$ contains a cycle of length three, i.e., $(\{a\},\{b\}),(\{b\},\{c\}),(\{c\},\{a\}) \in R_{F}$.

The following lemma characterizes WARP.

Lemma 1.1. F satisfies $W A R P$ if and only if for all $X, Y \in \mathcal{A}$ such that $Y \subseteq X$, $F(X)=F(Y \cup F(X))$. 
Proof. Let $F$ satisfy WARP and let $X, Y \in \mathcal{A}$ with $Y \subseteq X$. Suppose that $F(X) \neq F(Y \cup F(X))$. Since $F(Y \cup F(X)) \subseteq X$, the definition of $R_{F}$ implies $(F(X), F(Y \cup F(X))) \in R_{F}$; and since $F(X) \subseteq Y \cup F(X)$, we similarly obtain $(F(Y \cup F(X)), F(X)) \in R_{F}$. This violates WARP, and therefore, $F(X)=F(Y \cup F(X))$.

Next, let for all $X \in \mathcal{A}$ and all $Y \subseteq X, F(X)=F(Y \cup F(X))$. Let distinct $V, W \in \mathcal{A}$ such that $(V, W),(W, V) \in R_{F}$. We derive a contradiction as follows. Since $(V, W) \in R_{F}$, there exists $Z \in \mathcal{A}$ such that $F(Z)=V$ and $W \subseteq Z$. Similarly, since $(W, V) \in R_{F}$, there exists $Z^{\prime} \in \mathcal{A}$, such that $F\left(Z^{\prime}\right)=W$ and $V \subseteq Z^{\prime}$. Therefore, $W \subseteq Z$ and $V \subseteq Z^{\prime}$ imply $F(Z)=F(W \cup F(Z))=F(W \cup V)=$ $F\left(F\left(Z^{\prime}\right) \cup V\right)=F\left(Z^{\prime}\right)$; thus $V=W$, a contradiction.

An immediate consequence of Lemma 1.1 is that if $F$ satisfies WARP then it is a projection, a fact also established in Brand and Harrenstein (2011).

Corollary 1.1. Let $F$ satisfy WARP. Then, for all $X \in \mathcal{A}, F^{2}(X)=F(X)$.

Proof. By Lemma 1.1, for all pairs $X, Y \in \mathcal{A}$ such that $Y \subseteq X, F(X)=F(Y \cup$ $F(X))$. Choosing $Y=F(X)$ implies $F^{2}(X)=F(X)$.

Notice that the reverse is not true, as illustrated in the following example.

Example 1.2. Let $A=[0,1]$ and define $F$ as follows.

$$
F(X)= \begin{cases}\{1\} & \text { if } X=[0,1] \\ X & \text { otherwise }\end{cases}
$$

Clearly $F^{2}(X)=F(X)$. Next, consider sets $[0,1]$ and $\left[\frac{1}{2}, 1\right]$. Since $\left[\frac{1}{2}, 1\right] \subseteq$ $[0,1]$ and $F([0,1])=\{1\},\left(\{1\},\left[\frac{1}{2}, 1\right]\right) \in R_{F}$; in addition, since $\{1\} \subseteq\left[\frac{1}{2}, 1\right]$ and $F\left(\left[\frac{1}{2}, 1\right]\right)=\left[\frac{1}{2}, 1\right],\left(\left[\frac{1}{2}, 1\right],\{1\}\right) \in R_{F}$. Therefore, $F$ violates WARP. Note that a similar example with a finite $A$ can easily be constructed.

Brand and Harrenstein (2011) use the expression 'set-rationalizablity' instead of WARP and show that this is equivalent to a set-valued version of Chernoff's condition, which we now define.

Condition $\hat{\boldsymbol{\alpha}}$. For all $X, Y \in \mathcal{A}$, if $F(X \cup Y) \subseteq X \cap Y$, then $F(X \cup Y)=F(X)$.

It follows that condition $\hat{\alpha}$ is equivalent to the condition in Lemma 1.1. This is not hard to show directly, witnessing the following lemma, which together with Lemma 1.1 provides an alternative proof of Theorem 2 in Brand and Harrenstein (2011). 
Lemma 1.2. F satisfies condition $\hat{\alpha}$ if and only if for all $X, Y \in \mathcal{A}$ such that $Y \subseteq X, F(X)=F(Y \cup F(X))$.

Proof. Let $F$ satisfy condition $\hat{\alpha}$ and $X, Y \in \mathcal{A}$ with $Y \subseteq X$. Let $X^{\prime}=X$ and $Y^{\prime}=Y \cup F(X)$. Then $F\left(X^{\prime} \cup Y^{\prime}\right)=F(X) \subseteq X^{\prime} \cap Y^{\prime}$ and condition $\hat{\alpha}$ imply $F\left(X^{\prime} \cup Y^{\prime}\right)=F\left(Y^{\prime}\right)$. Therefore, $F(X)=F(Y \cup F(X))$.

Next, let $X, Y \in \mathcal{A}$ with $F(X \cup Y) \subseteq X \cap Y$. In addition, let $X^{\prime}=X \cup Y$ and $Y^{\prime}=X \backslash F(X \cup Y)$. Then, assuming that for all $X, Y \in \mathcal{A}$ such that $Y \subseteq X$, $F(X)=F(Y \cup F(X))$, implies that $F(X \cup Y)=F\left(X^{\prime}\right)=F\left(Y^{\prime} \cup F\left(X^{\prime}\right)\right)=$ $F((X \backslash F(X \cup Y)) \cup F(X \cup Y))=F(X)$, where the last equality follows since $F(X \cup Y) \subseteq X \cap Y \subseteq X$. Therefore, condition $\hat{\alpha}$ is satisfied.

\subsubsection{Weak independence of irrelevant alternatives}

The next property requires, for each set $X$ and for every subset of $X$ that has a nonempty intersection with $F(X)$, only alternatives from $F(X)$ to be chosen.

Weak independence of irrelevant alternatives (WIIA). For all $X, Y \in \mathcal{A}$ such that $Y \subseteq X$, if $F(X) \cap Y \neq \emptyset$, then $F(Y) \subseteq F(X)$.

Example 1.3 below shows that WIIA does not imply WARP. The reverse does not hold either, as shown in Example 1.4 that follows.

Example 1.3. Let $A=\mathbb{N}$ and define $F$ by

$$
F(X)= \begin{cases}X & \text { if }|X|=1 \text { or } X \text { is infinite } \\ X \backslash\{\max (X)\} & \text { if } 1<|X|<\infty\end{cases}
$$

Let $Y \subseteq X$ such that $F(X) \cap Y \neq \emptyset$. If $X$ is infinite, then trivially $F(Y) \subseteq$ $X=F(X)$. If $X$ is finite and $|Y|=1$, then $F(Y)=Y \cap F(X)=Y$. Otherwise, $F(Y) \subseteq Y \cap F(X)$. Hence, $F$ satisfies WIIA. However, let $X=\{1,2,3\}$ and $Y=\{1,2\}$. Then $F(X)=\{1,2\}$ implies $(\{1,2\},\{1\}) \in R_{F}$, while $F(Y)=\{1\}$ implies $(\{1\},\{1,2\}) \in R_{F}$. Hence, $F$ does not satisfy WARP. Note that these statements also hold for finite $A=\{1,2, \ldots, n\}$ with $n \geq 3$.

Example 1.4. Let $A=[0,1]$ and define $F$ by

$$
F(X)= \begin{cases}X \backslash\{0\} & \text { if } X \subseteq[0,1] \text { with } X \cap\left(\frac{1}{2}, 1\right] \neq \emptyset \\ X & \text { otherwise. }\end{cases}
$$


Then $F([0,1])=(0,1]$ whereas $F\left(\left[0, \frac{1}{2}\right]\right)=\left[0, \frac{1}{2}\right]$; hence, $F$ does not satisfy WIIA. By using Lemma 1.1 it is straightforward that $F$ satisfies WARP. The example can be easily adapted to a finite $A$.

Thus, there is no direct logical relation between WIIA and WARP. However, if $F$ satisfies WIIA then the restriction of $R_{F}$ to singletons has no cycles of length two. This is not surprising since WIIA reduces to the classical IIA for single-valued choice correspondences. For completeness, we provide the simple proof. Let $r_{F}$ denote this restriction, i.e., $(\{x\},\{y\}) \in r_{F}$ for distinct $x, y \in A$ if there is $Z \subseteq A$ such that $F(Z)=\{x\}$ and $y \in Z$.

Lemma 1.3. Let F satisfy WIIA. Then $r_{F}$ has no cycles of length two.

Proof. Let distinct $\{x\},\{y\} \in \mathcal{A}$ and assume that $(\{x\},\{y\}) \in r_{F}$ and $(\{y\},\{x\}) \in$ $r_{F}$ to derive a contradiction. Since $(\{x\},\{y\}) \in r_{F}$, there exists $X \in \mathcal{A}$ such that $F(X)=\{x\}$ and $y \in X$. Similarly, there exists $Y \in \mathcal{A}$ such that $x \in Y$ and $F(Y)=\{y\}$. Since $\{x, y\} \subseteq X$ and $F(X) \cap\{x, y\} \neq \emptyset$, WIIA implies $F(x, y) \subseteq F(X) \cap\{x, y\}=\{x\}$; hence, $F(x, y)=\{x\}$. Similarly, one obtains $F(x, y)=\{y\}$. Since $x \neq y$ this is a contradiction.

If we add the condition that $F$ be a projection, i.e. $F^{2}=F$, then WIIA implies WARP.

Lemma 1.4. Let $F$ satisfy WIIA and $F^{2}=F$. Then, $F$ satisfies WARP.

Proof. Let $X, Y, Z, Z^{\prime} \in \mathcal{A}$ such that $X, Y \subseteq Z, X, Y \subseteq Z^{\prime}, F(Z)=X$, and $F\left(Z^{\prime}\right)=Y$. By WIIA we have both $F(X \cup Y) \subseteq F\left(Z^{\prime}\right)=Y$ and $F(X \cup Y) \subseteq$ $F(Z)=X$, so that $F(X \cup Y) \subseteq X \cap Y$. This implies $X \cap Y=F(Z) \cap F\left(Z^{\prime}\right) \neq$ $\emptyset$. Hence, by Lemma 1.11, $F(Z) \cup F\left(Z^{\prime}\right)=F\left(F(Z) \cup F\left(Z^{\prime}\right)\right)$, and therefore $X \cup Y=F(X \cup Y) \subseteq X \cap Y$, which implies $X=Y$. We conclude that $F$ satisfies WARP.

The converse of Lemma 1.4 does not hold. If $F$ satisfies WARP then by Corollary 1.1 it is a projection, but Example 1.4 shows that WIIA does not have to hold.

The following result shows that if $F$ satisfies WIIA, then so does every $n$-fold composition of $F$ with itself. It will be useful later on.

Lemma 1.5. Let $F$ satisfy WIIA and let $n \in \mathbb{N}$ with $n \geq 2$. Then $F^{n}$ satisfies WIIA. 
Proof. The proof is based on induction: $F^{1}=F$ satisfies WIIA, and assume that $F^{k}$ satisfies WIIA for every $k=2, \ldots, n-1$. Let $X, Y \in \mathcal{A}$ with $Y \subseteq X$ and $F^{n}(X) \cap Y \neq \emptyset$. We have to show that $F^{n}(Y) \subseteq F^{n}(X)$.

Note that for every $k \in\{1, \ldots, n-1\}, F^{k}(X) \cap Y \neq \emptyset$ and the induction assumption imply that $F^{k}(Y) \subseteq F^{k}(X)$ and thus, that

$$
F^{\ell}(X) \cap F^{m}(Y) \neq \emptyset \text { for all } \ell, m \in\{1, \ldots, n-1\} .
$$

We now first prove that

$$
F^{n}(X) \cap F^{k}(Y) \neq \emptyset \text { for every } k=0, \ldots, n-1
$$

where $F^{0}(Y)=Y$. The proof of (1.2) is by induction. By assumption, $F^{n}(X) \cap$ $F^{0}(Y)=F^{n}(X) \cap Y \neq \emptyset$. Let $1 \leq \ell \leq n-1$ and assume that $F^{n}(X) \cap F^{k}(Y) \neq \emptyset$ for every $k=1, \ldots, \ell-1$. We show that $F^{n}(X) \cap F^{\ell}(Y) \neq \emptyset$. First, since $\emptyset \neq F^{n-1}(X) \cap F^{\ell-1}(Y) \subseteq F^{n-1}(X)$ by $(1.1)$, and $F^{n}(X) \cap\left(F^{n-1}(X) \cap F^{\ell-1}(Y)\right)=$ $F^{n}(X) \cap F^{\ell-1}(Y) \neq \emptyset$ by the induction assumption for this part, WIIA of $F$ implies

$$
F\left(F^{n-1}(X) \cap F^{\ell-1}(Y)\right) \subseteq F^{n}(X)
$$

Second, since $\emptyset \neq F^{n-1}(X) \cap F^{\ell-1}(Y) \subseteq F^{\ell-1}(Y)$ by $(1.1)$, and $F^{\ell}(Y) \cap\left(F^{n-1}(X) \cap\right.$ $\left.F^{\ell-1}(Y)\right)=F^{\ell}(Y) \cap F^{n-1}(X) \neq \emptyset$ by (1.1), WIIA of $F$ implies

$$
F\left(F^{n-1}(X) \cap F^{\ell-1}(Y)\right) \subseteq F^{\ell}(Y)
$$

By (1.3) and (1.4), $F^{n}(X) \cap F^{\ell}(Y) \neq \emptyset$, which completes the proof of (1.2).

Now, since $F^{n-1}(X) \cap Y \neq \emptyset$, the assumed WIIA of $F^{n-1}$ implies $F^{n-1}(Y) \subseteq$ $F^{n-1}(X)$. Since by $(1.2)$ we have $F^{n}(X) \cap F^{n-1}(Y) \neq \emptyset$, WIIA of $F$ implies $F^{n}(Y) \subseteq F^{n}(X)$. This completes the proof of the lemma.

If $A$ is finite, then there exists $n \in \mathbb{N}$ such that $F^{\ell}=F^{n}$ for all $\ell \geq n$. In this case, Lemma 1.5 implies that if $F$ satisfies WIIA, then so does $F^{n}$. If $A$ is infinite, such an $n$ does not necessarily exist. However, we may define $F^{\infty}$ by $F^{\infty}(X)=\cap_{n \in \mathbb{N}} F^{n}(X)$ for every $X \in \mathcal{A}$, assuming that this set is nonempty for every $X \in \mathcal{A}$. The following example shows that this condition is not necessarily satisfied if $F$ satisfies WIIA. 
Example 1.5. Let $A=[0,1]$ and for every $X \in \mathcal{A}$, let $x$ be the maximal number in $\mathbb{N} \cup\{0\}$ such that $X \subseteq\left[0, \frac{1}{2^{x}}\right]$. We define $F$ by

$$
F(X)= \begin{cases}X \backslash\left(\frac{1}{2^{x+1}}, \frac{1}{2^{x}}\right] & \text { if } X \backslash\left(\frac{1}{2^{x+1}}, \frac{1}{2^{x}}\right] \neq \emptyset \\ X & \text { otherwise. }\end{cases}
$$

It is easy to check that $F$ satisfies WIIA. However, $\cap_{n \in \mathbb{N}} F^{n}(A \backslash\{0\})=$ $\cap_{n \in \mathbb{N}}\left(0, \frac{1}{2^{n}}\right]=\emptyset$.

Remark 1.1. If $F$ satisfies WIIA and $F^{\infty}$ is well defined, then it follows from Lemma 1.5 that $F^{\infty}$ satisfies WIIA.

\subsubsection{Independence of irrelevant alternatives}

The next property was first proposed by Nash (cf. Shubik, 1982), and also appears in Chernoff (1954) and Arrow (1959).

Independence of irrelevant alternatives (IIA). For all $X, Y \in \mathcal{A}$ such that $Y \subseteq X$, if $F(X) \cap Y \neq \emptyset$, then $F(Y)=F(X) \cap Y$.

For single-valued choice, IIA is equivalent to WARP as long as the domain of choice sets is closed under intersection, more precisely, if every nonempty intersection is in the domain. In the present context, this is no longer true: IIA implies WARP, as shown by the lemma below, but not the other way around, as shown by the example that follows.

Lemma 1.6. Let F satisfy IIA. Then F satisfies WARP.

Proof. Let $X, Y \in \mathcal{A}$ such that $(X, Y) \in R_{F}$. Then there is $Z \in \mathcal{A}$ such that $F(Z)=X$ and $Y \subseteq Z$. We have to show that $(Y, X) \notin R_{F}$. This is true if $F(V)=Y$ for no $V \in \mathcal{A}$ with $X \subseteq V$. Now suppose $F(V)=Y$ for some $V \in \mathcal{A}$ with $X \subseteq V$. If $X \subseteq V$ then by IIA applied to $Z \cap V$ we have both $F(Z \cap V)=X$ and $F(Z \cap V)=Y$, an impossibility since $X \neq Y$. Therefore, $X \nsubseteq V$, which completes the proof.

Example 1.6. Let $A=\{a, b, c\}$ and define $F$ by

$$
F(X)= \begin{cases}\{a\} & \text { if } X \in\{\{a, b\},\{a, c\}\} \\ \{b\} & \text { if } X=\{b, c\} \\ X & \text { otherwise. }\end{cases}
$$


It can be easily checked that $F$ satisfies WARP. However, since $\{a, b\} \subseteq\{a, b, c\}$ and $\{a, b\} \cap F(\{a, b, c\}) \neq \emptyset$, it follows that $F(\{a, b\})=\{a\}$ violates IIA. By partitioning a set in three nonempty subsets, the example can be easily adapted to an infinite $A$.

In order to obtain a characterization of IIA we introduce the following condition on the revealed preference relation of a choice correspondence: if $X$ is revealed preferred to $Y$, then every subset of $X$ that includes all alternatives in $X \cap Y$, is also revealed preferred to $Y$.

Preference Axiom (PA) For all distinct $X, Y, Z \in \mathcal{A}$ such that $X \cap Y \subseteq$ $Z \subseteq X$, if $(X, Y) \in R_{F}$, then $(Z, Y) \in R_{F}$.

This axiom can be interpreted as expressing that what really makes $X$ (revealed) preferred to $Y$ is the intersection of $X$ and $Y$. Indeed, the axiom implies that if $X$ is preferred to $Y$ then $X \cap Y$, if nonempty, is preferred to $Y$.

We have the following characterization of IIA.

Theorem 1.1. F satisfies IIA if and only if it satisfies WARP and PA.

Proof. (only if part) Let $F$ satisfy IIA. Then $F$ satisfies WARP by Lemma 1.6. We show that $F$ satisfies PA. Let distinct $X, Y, Z \in \mathcal{A}$ such that $X \cap Y \subseteq Z \subseteq X$ and $(X, Y) \in R_{F}$. By IIA of $F$ and $(X, Y) \in R_{F}, F(X \cup Y)=X$. Since $Z \cup Y \subseteq X \cup Y$ and $F(X \cup Y) \cap(Z \cup Y)=X \cap(Z \cup Y)=(X \cap Z) \cup(X \cap Y)=Z \neq \emptyset$, IIA of $F$ implies $F(Z \cup Y)=Z$. Therefore, $(Z, Y) \in R_{F}$.

(if part) Let $F$ satisfy WARP and PA. We show that $F$ satisfies IIA. Let $X, Y \in \mathcal{A}$ with $Y \subseteq X$ and $F(X) \cap Y \neq \emptyset$. Suppose that $F(Y) \neq F(X) \cap Y$. Since $F(Y) \subseteq Y \subseteq X$, it follows that $(F(X), F(Y)) \in R_{F}$. In addition, $(F(X) \cap F(Y)) \subseteq$ $(F(X) \cap Y) \subseteq F(X)$, so that by PA, $(F(X) \cap Y, F(Y)) \in R_{F}$. But also, $F(Y) \subseteq Y$ and $F(X) \cap Y \subseteq Y$ imply $(F(Y), F(X) \cap Y) \in R_{F}$. This violates WARP; therefore $F(Y)=F(X) \cap Y$. Thus, $F$ satisfies IIA.

In Example 1.6, $F$ satisfies WARP but not IIA. Hence it follows from Theorem 1.1 that $F$ does not satisfy PA either. This can also be easily established directly. E.g., let $X=\{a, b, c\}, Y=\{b\}$, and $Z=\{a, b\}$, then $(X, Y) \in R_{F}$ but $(Z, Y) \notin$ $R_{F}$.

The next example shows that PA does not imply IIA or WARP. 
Example 1.7. Let $A=\{a, b, c, d\}$ and define $F$ by

$$
F(X)= \begin{cases}\{a, b\} & \text { if } X=\{a, b, c, d\} \\ \{a\} & \text { if } X \subsetneq\{a, b, c, d\} \text { and } a \in X \\ \{b\} & \text { if } X \subseteq\{b, c, d\} \text { and } b \in X \\ \{c\} & \text { if } X \subseteq\{c, d\} \text { and } c \in X \\ \{d\} & \text { if } X=\{d\} .\end{cases}
$$

It is straightforward to show that $F$ satisfies PA. Since $\{a, b\} \subseteq\{a, b, c, d\},\{a, b\} \cap$ $F(\{a, b, c, d\}) \neq \emptyset$, and $F(\{a, b\})=\{a\}$, it follows that $F$ does not satisfy IIA and by Theorem 1.1, it also does not satisfy WARP.

In Section 1.2.3 we have already seen that WIIA and WARP are logically independent. The same is true for WIIA and PA since in Example 1.3, F satisfies WIIA but not PA. The following example shows that PA does not imply WIIA.

Example 1.8. Let $A=\{a, b, c\}$ and define $F$ by

$$
F(X)= \begin{cases}\{a\} & \text { if } X=A \\ X & \text { otherwise }\end{cases}
$$

In this case, $F$ does not satisfy WIIA since $F(\{a, b\})=\{a, b\} \nsubseteq\{a\}=F(A) \cap$ $\{a, b\}$. To show that $F$ satisfies PA, let $X, Y, Z$ as in the statement of PA and $V \in \mathcal{A}$ such that $F(V)=X$ and $Y \subseteq V$. If $|X|=1$ then either $V=X$ and then $Z=X=Y$, a contradiction since $Z \neq Y$; or $V=A$, which implies $X=\{a\}$ and therefore $Z=\{a\}=X$, so that $(Z, Y) \in R_{F}$. If $|X|=2$, then $V=X$ and $Y \subseteq X$ with $|Y|=1$; this implies $Z=X$ and thus $(Z, Y) \in R_{F}$.

Theorem 1.2. Let $F$ satisfy IIA. Then $R_{F}$ is transitive and acyclic.

Proof. By Theorem 1.1, $F$ satisfies WARP. It is sufficient to prove that $R_{F}$ is transitive, since with WARP this implies acyclicity. Let distinct $X_{1}, X_{2}, X_{3} \in \mathcal{A}$ with $\left(X_{1}, X_{2}\right),\left(X_{2}, X_{3}\right) \in R_{F}$. We prove that $\left(X_{1}, X_{3}\right) \in R_{F}$. Let $Z=X_{1} \cup X_{2} \cup$ $X_{3}$. We consider two cases for $F(Z)$.

If $F(Z) \cap X_{3}=\emptyset$ then $F(Z) \subseteq X_{1} \cup X_{2}$. If $F(Z) \neq X_{1}$ then $\left(F(Z), X_{1}\right) \in R_{F}$. On the other hand, since $\left(X_{1}, X_{2}\right) \in R_{F}$ there is $Z_{1} \in \mathcal{A}$ such that $F\left(Z_{1}\right)=X_{1}$ whereas $X_{2} \subseteq Z_{1}$; in particular, this implies $\left(X_{1}, F(Z)\right) \in R_{F}$, so that WARP is violated. Hence, in this case, $F(Z)=X_{1}$ and therefore $\left(X_{1}, X_{3}\right) \in R_{F}$. 
If $F(Z) \cap X_{3} \neq \emptyset$ then $F\left(X_{2} \cup X_{3}\right)=F(Z) \cap\left(X_{2} \cup X_{3}\right)$ by IIA, hence $F\left(X_{2} \cup\right.$ $\left.X_{3}\right) \cap X_{3} \neq \emptyset$. This implies $\left(F\left(X_{2} \cup X_{3}\right), X_{2}\right) \in R_{F}$. On the other hand, by a similar argument as in the first case, $\left(X_{2}, X_{3}\right) \in R_{F}$ implies $\left(X_{2}, F\left(X_{2} \cup X_{3}\right)\right) \in R_{F}$, violating WARP. Hence, in this case, $\left(X_{1}, X_{3}\right) \in R_{F}$, which concludes the proof of the theorem.

For (single-valued) choice functions on a domain that is closed under nonempty intersection, II $A$ and $W A R P$ are equivalent, but do not necessarily imply acyclicity of the revealed preference relation (e.g., Gale, 1960; Peters and Wakker, 1994). ${ }^{3}$ In our case, IIA is stronger than WARP (cf. Theorem 1.1), and implies transitivity and acyclicity of the revealed preference relation.

The converse of Theorem 1.2 does not hold: the revealed preference relation $R_{F}$ of the choice correspondence $F$ in Example 1.7 is transitive and acyclic, but $F$ does not satisfy IIA.

\subsection{Weak and strong sets}

In this section we introduce two collections of choice sets in relation to a given choice correspondence. We will show, in this section and the next one, that these collections are relevant for choice correspondences satisfying WIIA and IIA, respectively.

\subsubsection{Weak sets}

A set $S \in \mathcal{A}$ is a weak set if the following holds. For all sets where some alternatives of $S$ are chosen, only alternatives of $S$ are chosen; and in addition, if all alternatives of $S$ are available, then all alternatives of $S$ are chosen.

Weak sets. $S \in \mathcal{A}$ is a weak set at $F$ if for all $X \in \mathcal{A}$ for which $F(X) \cap S \neq \emptyset$, we have:

(i) $F(X) \subseteq S$

(ii) if $S \subseteq X$, then $F(X)=S$.

The set of weak sets at $F$ is denoted by $\mathcal{S}_{F}$. By $R_{\mathcal{S}_{F}}=\left\{(X, Y) \in R_{F} \mid X, Y \in \mathcal{S}_{F}\right\}$ we denote the restriction of $R_{F}$ to $\mathcal{S}_{F}$.

We show below that the elements of $\mathcal{S}_{F}$ are pairwise disjoint and then, in Lemma 1.8 which follows, that $R_{\mathcal{S}_{F}}$ behaves well if $F$ satisfies WIIA.

\footnotetext{
${ }^{3}$ That is, they do not necessarily imply that $F$ satisfies the so-called Strong Axiom of Revealed Preference.
} 
Lemma 1.7. Let distinct $S, T \in \mathcal{S}_{F}$. Then, $S \cap T=\emptyset$.

Proof. Let $Z=S \cup T$. Without loss of generality assume that $F(Z) \cap S \neq \emptyset$. Then $S \in \mathcal{S}_{F}$ and $S \subseteq Z$ imply $F(Z)=S$. If $S \cap T \neq \emptyset$, then $F(Z) \cap T \neq \emptyset$; hence $T \in \mathcal{S}_{F}$ and $T \subseteq Z$ imply $F(Z)=T=S$. This contradicts $S \neq T$. Consequently, $S \cap T=\emptyset$.

Lemma 1.8. Let F satisfy WIIA. Then $R_{\mathcal{S}_{F}}$ is complete and acyclic.

Proof. Let $S, T \in \mathcal{S}_{F}$ with $S \neq T$. By the definition of $\mathcal{S}_{F}, F(S \cup T) \in\{S, T\}$; hence, $R_{\mathcal{S}_{F}}$ is complete. Without loss of generality assume that $F(S \cup T)=S$. We show that $(T, S) \notin R_{\mathcal{S}_{F}}$, which implies that $R_{\mathcal{S}_{F}}$ has no cycles of length 2 . To show this, let $Z \in \mathcal{A}$ with $S \cup T \subseteq Z$. If $(S \cup T) \cap F(Z) \neq \emptyset$, then by WIIA, $S=F(S \cup T) \subseteq(S \cup T) \cap F(Z)$. Since $S \in \mathcal{S}_{F}$, this implies that $F(Z)=S$. Since $Z$ was arbitrary, we have $(T, S) \notin R_{\mathcal{S}_{F}}$.

In order to show that $R_{\mathcal{S}_{F}}$ has also no cycles of length larger than 2 , let $n \geq 2$ and $S^{0}, \ldots, S^{n} \in \mathcal{S}_{F}$ with $\left(S^{i}, S^{i+1}\right) \in R_{\mathcal{S}_{F}}$ for each $i=0, \ldots, n-1$. Since $R_{\mathcal{S}_{F}}$ has no cycles of length 2 , it is sufficient to show that $\left(S^{0}, S^{n}\right) \in R_{\mathcal{S}_{F}}$. Since $S^{i} \in \mathcal{S}_{F}$ for every $i=0, \ldots, n$, we have $F\left(\cup_{i=0}^{n} S^{i}\right)=S^{j}$ for some $j \in\{0, \ldots, n\}$. If $j \neq 0$, then $\left(S^{j-1}, S^{j}\right),\left(S^{j}, S^{j-1}\right) \in R_{\mathcal{S}_{F}}$, so that we have a cycle of length 2. Hence, $j=0$, which implies in particular $\left(S^{0}, S^{n}\right) \in R_{\mathcal{S}_{F}}$.

\subsubsection{Strong sets}

A set $S \in \mathcal{A}$ is a strong set (of alternatives) if the following holds. For all sets where some alternatives of $S$ are chosen, all the available alternatives of $S$ are chosen, and only these.

Strong sets. $S \in \mathcal{A}$ is a strong set at $F$ if for all $X \in \mathcal{A}$ for which $F(X) \cap S \neq \emptyset$, we have $F(X)=S \cap X$. The set of strong sets induced by $F$ is denoted by $\tilde{\mathcal{S}}_{F}$. By $R_{\tilde{\mathcal{S}}_{F}}=\left\{(X, Y) \in R_{F} \mid X, Y \in \tilde{\mathcal{S}}_{F}\right\}$ we denote the restriction of $R_{F}$ to $\tilde{\mathcal{S}}_{F}$.

Since, clearly, $\tilde{\mathcal{S}}_{F} \subseteq \mathcal{S}_{F}$, Lemmas 1.7 and 1.8 also hold for the set of strong sets. For easy reference we formulate the following lemma.

Lemma 1.9. The elements of $\tilde{\mathcal{S}}_{F}$ are pairwise disjoint, and $R_{\tilde{\mathcal{S}}_{F}}$ is complete and acyclic. 


\subsection{Partitions induced by WIIA and IIA}

In this section we discuss partitions of the set of alternatives related to WIIA and IIA of a choice correspondence. These are the sets of weak and strong sets introduced in the preceding section.

\subsubsection{Weak sets and WIIA}

Before proceeding with our main result, we first present to lemmas that are used in its proof. Specifically, given a choice correspondence $F$, Lemma 1.10 shows some consequences for sets of choice sets (i.e., some $\mathcal{Z} \subseteq \mathcal{A}$ ) with common chosen alternatives in all these choice sets, if WIIA or IIA are satisfied by $F$. Then, Lemma 1.11 strengthens this result in the case where $F^{2}=F$.

Lemma 1.10. Let $F$ be a choice correspondence and $\emptyset \neq \mathcal{Z} \subseteq \mathcal{A}$ such that $\cap_{Z \in \mathcal{Z}} F(Z) \neq \emptyset$. Then the following statements hold:

(i) If $F$ satisfies WIIA then $\cup_{Z \in \mathcal{Z}} F(Z) \subseteq F\left(\cup_{Z \in \mathcal{Z}} Z\right)$.

(ii) If $F$ satisfies IIA then $\cup_{Z \in \mathcal{Z}} F(Z)=F\left(\cup_{Z \in \mathcal{Z}} Z\right)$.

Proof. (i) Let $x \in \cap_{Z \in \mathcal{Z}} F(Z)$. Since $F\left(\cup_{Z \in \mathcal{Z}} Z\right) \subseteq \cup_{Z \in \mathcal{Z}} Z$, there is $Z^{\prime} \in \mathcal{Z}$ such that $Z^{\prime} \cap F\left(\cup_{Z \in \mathcal{Z}} Z\right) \neq \emptyset$, so that $F\left(Z^{\prime}\right) \subseteq F\left(\cup_{Z \in \mathcal{Z}} Z\right)$ by WIIA. Hence, $x \in F\left(\cup_{Z \in \mathcal{Z}} Z\right)$, so that $Z^{\prime} \cap F\left(\cup_{Z \in \mathcal{Z}} Z\right) \neq \emptyset$ for all $Z^{\prime} \in \mathcal{Z}$, and hence $F\left(Z^{\prime}\right) \subseteq$ $F\left(\cup_{Z \in \mathcal{Z}} Z\right)$ for all $Z^{\prime} \in \mathcal{Z}$ by WIIA. This proves part (i).

(ii) Let $F$ satisfy IIA. Using similar arguments as in part (i) now implies that $F\left(Z^{\prime}\right)=F\left(\cup_{Z \in \mathcal{Z}} Z\right) \cap Z^{\prime}$ for all $Z^{\prime} \in \mathcal{Z}$ by IIA. This proves part (ii).

Lemma 1.11. Let $F$ be a choice correspondence satisfying WIIA and $F^{2}=F$. Let $\emptyset \neq \mathcal{Z} \subseteq \mathcal{A}$ such that $\cap_{Z \in \mathcal{Z}} F(Z) \neq \emptyset$. Then $\cup_{Z \in \mathcal{Z}} F(Z)=F\left(\cup_{Z \in \mathcal{Z}} F(Z)\right)$.

Proof. Let $x \in \cap_{Z \in \mathcal{Z}} F(Z)$. We first show that $x \in F\left(\cup_{Z \in \mathcal{Z}} F(Z)\right)$. Since $F\left(\cup_{Z \in \mathcal{Z}} F(Z)\right) \subseteq \cup_{Z \in \mathcal{Z}} F(Z)$, there is a $Z^{\prime} \in \mathcal{Z}$ such that $F\left(Z^{\prime}\right) \cap F\left(\cup_{Z \in \mathcal{Z}} F(Z)\right) \neq$ $\emptyset$. Then WIIA and $F^{2}=F$ imply $x \in F\left(Z^{\prime}\right)=F^{2}\left(Z^{\prime}\right) \subseteq F\left(\cup_{Z \in \mathcal{Z}} F(Z)\right)$.

Since, thus, $x \in F\left(\cup_{Z \in \mathcal{Z}} F(Z)\right)$ it follows that $F\left(Z^{\prime}\right) \cap F\left(\cup_{Z \in \mathcal{Z}} F(Z)\right) \neq \emptyset$ for all $Z^{\prime} \in \mathcal{Z}$ and hence by WIIA and $F^{2}=F$ that $F\left(Z^{\prime}\right)=F^{2}\left(Z^{\prime}\right) \subseteq F\left(\cup_{Z \in \mathcal{Z}} F(Z)\right)$ for all $Z^{\prime} \in \mathcal{Z}$. Hence, $\cup_{Z \in \mathcal{Z}} F(Z) \subseteq F\left(\cup_{Z \in \mathcal{Z}} F(Z)\right)$.

Our main result follows.

Theorem 1.3. Let $F$ satisfy WIIA and let $F=F^{2}$. Then $\mathcal{S}_{F}$ is a partition of $A$ and $R_{\mathcal{S}_{F}}$ is complete and acyclic. 
Proof. Let $x \in A$. In view of Lemmas 1.7 and 1.8 we only still have to prove that there is an $S \in \mathcal{S}_{F}$ such that $x \in S$. Define $\mathcal{Z}=\{Z \in \mathcal{A} \mid x \in F(Z)\}$ and $S=\cup_{Z \in \mathcal{Z}} F(Z)$. It is sufficient to prove that $S \in \mathcal{S}_{F}$. To this end, let $X \in \mathcal{A}$ such that $F(X) \cap S \neq \emptyset$. This implies, in particular, that there is $Z^{\prime} \in \mathcal{Z}$ such that $F(X) \cap F\left(Z^{\prime}\right) \neq \emptyset$. By Lemma 1.10(i) we obtain $F(X) \cup F\left(Z^{\prime}\right) \subseteq F\left(X \cup Z^{\prime}\right)$, which implies $x \in F\left(X \cup Z^{\prime}\right)$ and therefore $X \cup Z^{\prime} \in \mathcal{Z}$. Hence $F(X) \subseteq F\left(X \cup Z^{\prime}\right) \subseteq S$. Finally, assume additionally that $S \subseteq X$. We show that $F(X)=S$, which then completes the proof. By Lemma 1.11, $S=\cup_{Z \in \mathcal{Z}} F(Z)=F\left(\cup_{Z \in \mathcal{Z}} F(Z)\right)=F(S)$. Since $S \subseteq X$ and $F(X) \cap S \neq \emptyset$, WIIA implies $F(S) \subseteq F(X)$ and therefore $S \subseteq F(X)$. Together with $F(X) \subseteq S$, this implies $F(X)=S$.

Theorem 1.3 thus states that a WIIA choice correspondence that is, moreover, a projection, induces a partition of the set of alternatives such that the alternatives assigned to every choice set lie in exactly one element of this partition. Moreover, if the choice set contains that partition element, then that element is assigned completely.

However, the converse of this result does not hold. Example 1.10 exhibits a projection $F$, where $\mathcal{S}_{F}$ is a partition of $A$ and $R_{\mathcal{S}_{F}}$ is complete and acyclic, that violates WIIA.

Two particular applications that follows from Theorem 1.3 are collected in the following corollary.

Corollary 1.2. Let F satisfy WIIA.

(i) If $A$ is finite and $m=|A|-1$, then $\mathcal{S}_{F^{m}}$ is a partition of $A$ and $R_{\mathcal{S}_{F}}$ is complete and acyclic.

(ii) If $F^{\infty}$ is well-defined and $F^{\infty}=F^{\infty} \circ F^{\infty}$, then $\mathcal{S}_{F}$ is a partition of $A$ and $R_{\mathcal{S}_{F} \infty}$ is complete and acyclic.

Proof. Statement (i) follows from Lemma 1.5 and Theorem 1.3. Statement (ii) follows from Remark 1.1 and Theorem 1.3.

The condition in Corollary 1.2(ii) that $F^{\infty}$ is a projection is not redundant as is illustrated by the following example.

Example 1.9. Let $A=\{-1,0\} \cup\left\{\frac{1}{2^{n}} \mid n \in \mathbb{N}\right\}$. Define $F$ by

$$
F(X)= \begin{cases}X \backslash\{\max \{x: x \in X\}\} & \text { if }|X|>1 \\ X & \text { otherwise. }\end{cases}
$$


Then $F^{\infty}$ is well-defined, and both $F$ and $F^{\infty}$ satisfy WIIA. Since $F^{\infty}(A)=$ $\{-1,0\}$ and $F^{\infty}(\{-1,0\})=\{-1\}$, we have $F^{\infty} \circ F^{\infty}(A)=\{-1\} \neq F^{\infty}(A)$. Hence, $F^{\infty} \circ F^{\infty} \neq F^{\infty}$.

The following lemma concludes our study of WIIA in this chapter.

\section{Lemma 1.12.}

(i) For finite $A$, let $F$ be a projection and satisfy WIIA, $S_{0}=\emptyset$, and $S_{i}=$ $F\left(A \backslash \cup_{k=0}^{i-1} S_{k}\right)$. Then, $\mathcal{S}_{F}=\left\{S_{1}, \ldots, S_{\ell}\right\}$ and $\left(S_{j}, S_{i}\right) \in R_{\mathcal{S}_{F}}$ whenever $j<i$.

(ii) For general $A$, let $\mathcal{T}$ be a partition of $A$ completely and acyclically ordered by $R$, and suppose moreover that for every $X \in \mathcal{A}$, the collection $\{S \in \mathcal{T} \mid S \cap X \neq$ $\emptyset\}$ has a maximal element $S_{X}$ according to $R$. Then, there exists a (not necessarily unique) projection $F$ satisfying WIIA, where $\mathcal{S}_{F}=\mathcal{T}$ and $R_{\mathcal{S}_{F}}=R$.

Proof. We prove statement (i) as follows: we first propose a partition $\mathcal{T}$ of $A$, then we show that $\mathcal{S}_{F}=\mathcal{T}$, and finally we show that $R_{\mathcal{S}_{F}}$ is complete and acyclic. The proof of statement (ii) is much simpler.

(i) Since $A$ is finite and for all $X \in \mathcal{A}, F(X) \subseteq X$ and $F(X) \neq \emptyset$, there exists a (finite) integer $\ell$, where $S_{\ell}=F\left(A \backslash \cup_{k=0}^{\ell-1} S_{k}\right)=A \backslash \cup_{k=0}^{\ell-1} S_{k}$. Let $\mathcal{T}=\left\{S_{1}, \ldots, S_{\ell}\right\}$; it follows that $\mathcal{T}$ partitions $A$.

Next, since $\mathcal{T}$ partitions $A$, for all $X \in \mathcal{A}$, there exists $\hat{k} \in\{1, \ldots, \ell\}$ such that $X \subseteq A \backslash \cup_{k=0}^{\hat{k}-1} S_{k}$ and $X \cap S_{\hat{k}} \neq \emptyset$; hence, WIIA implies $F(X) \subseteq S_{\hat{k}}$. Moreover, notice that $F$ being a projection implies $F\left(S_{\hat{k}}\right)=S_{\hat{k}}$; hence, if in addition $S_{\hat{k}} \subseteq X$, then it follows by WIIA that $F(X)=S_{\hat{k}}$. Therefore, $\mathcal{S}_{F}=\mathcal{T}$.

Finally, since $j<i \leq \ell$ implies that $S_{j}=F\left(A \backslash \cup_{k=0}^{j-1} S_{k}\right)$ and $S_{i} \subseteq A \backslash \cup_{k=0}^{i-1} S_{k}$, it follows that $\left(S_{j}, S_{i}\right) \in R_{F}$ which completes the proof.

(ii) Define $F$ such that for all $X \in \mathcal{A}, F(X)=X \cap S_{X}$. It is easy to verify that $F$ is a projection and satisfies WIIA (in fact it satisfies IIA). Moreover, it is clear that $\mathcal{S}_{F}=\mathcal{T}$ and $R_{\mathcal{S}_{F}}=R$.

\subsubsection{Strong sets and IIA}

The analogue of Theorem 1.3 is the following.

Theorem 1.4. Let F satisfy IIA. Then $\tilde{\mathcal{S}}_{F}$ is a partition of $A$ and $R_{\tilde{\mathcal{S}}_{F}}$ is complete and acyclic.

Proof. Let $x \in A$. In view of Lemma 1.9 we only still have to prove that there is an $S \in \tilde{\mathcal{S}}_{F}$ such that $x \in S$. Define $\mathcal{Z}=\{Z \in \mathcal{A} \mid x \in F(Z)\}$ and $S=F\left(\cup_{Z \in \mathcal{Z}} Z\right)$. By Lemma 1.10(ii) we have $S=\cup_{Z \in \mathcal{Z}} F(Z) \ni x$, so that it is sufficient to prove 
that $S \in \tilde{\mathcal{S}}_{F}$. To this end, let $X \in \mathcal{A}$ such that $F(X) \cap S \neq \emptyset$, then it is sufficient to prove that $F(X)=S \cap X$.

Since $F(X) \cap S \neq \emptyset$, Lemma 1.10(ii) implies $F(X) \cup S=F(X) \cup F\left(\cup_{Z \in \mathcal{Z}} Z\right)=$ $F\left(X \cup\left(\cup_{Z \in \mathcal{Z}} Z\right)\right)$. In particular, this implies that $F\left(X \cup\left(\cup_{Z \in \mathcal{Z}} Z\right)\right) \cap X \neq \emptyset$ so that by IIA we obtain $F(X)=F\left(X \cup\left(\cup_{Z \in Z} Z\right)\right) \cap X$. Thus, it follows that $F(X)=(F(X) \cup S) \cap X=F(X) \cup(S \cap X)$. Hence, $F(X) \supseteq S \cap X$. Therefore, it is sufficient to prove that $F(X) \subseteq S \cap X$.

By Lemma 1.10(ii), $F(X) \cap S \neq \emptyset$ implies $F(X) \cap\left(\cup_{Z \in \mathcal{Z}} F(Z)\right) \neq \emptyset$. Hence, for some $Z^{\prime} \in \mathcal{Z}, F(X) \cap F\left(Z^{\prime}\right) \neq \emptyset$. Thus, by Lemma 1.10(ii), $F\left(X \cup Z^{\prime}\right)=$ $F(X) \cup F\left(Z^{\prime}\right)$. It follows that $x \in F\left(X \cup Z^{\prime}\right)$ and thus, $X \cup Z^{\prime} \in \mathcal{Z}$. In addition, since $S=\cup_{Z \in \mathcal{Z}} F(Z), F\left(X \cup Z^{\prime}\right) \subseteq S$, and hence, $F(X) \cup F\left(Z^{\prime}\right) \subseteq S$. Therefore, $F(X) \subseteq S$ and trivially, $F(X) \subseteq S \cap X$.

Remark 1.2. For finite $A$, if $F$ satisfies IIA, then the set of strong sets $\tilde{\mathcal{S}}_{F}$ can be computed analogously as in Lemma 1.12(i). Conversely, for general $A$, let $\mathcal{T}$ be a partition of $A$ completely and acyclically ordered by $R$. Suppose moreover that for every $X \in \mathcal{A}$, the collection $\{S \in \mathcal{T} \mid S \cap X \neq \emptyset\}$ has a maximal element $S_{X}$ according to $R$. Then, it is easy to verify that $F(X)=X \cap S_{X}$ defines an IIA choice correspondence with $\tilde{\mathcal{S}}_{F}=\mathcal{T}$ as the set of strong sets with ordering $R_{\tilde{\mathcal{S}}_{F}}=R$. The uniqueness of $F$ can be shown easily by contradiction: assuming that for some $Y \in \mathcal{A}, F(Y) \neq Y \cap S_{Y}$, either implies that $F$ violates IIA, or that $F\left(S_{Y}\right) \neq S_{Y}$ and thus that $S_{Y} \notin \tilde{\mathcal{S}}_{F}$.

The logical converses of Theorems 1.3 and 1.4 do not hold. The following example describes a projection $F$ of which the sets of weak and strong sets coincide, are a partition of $A$, and are completely and acyclically ordered by $R_{F}$, but which does not satisfy WIIA.

Example 1.10. Let $F(A) \subsetneq A$ and for all $X \in \mathcal{A}$ define $F$ by

$$
F(X)= \begin{cases}F(A) & \text { if } F(A) \subseteq X \\ X & \text { if } X \subseteq F(A) \\ X \backslash F(A) & \text { otherwise. }\end{cases}
$$

It is straightforward to verify that $F$ is a projection and $\mathcal{S}_{F}=\tilde{\mathcal{S}}_{F}=\{F(A), A \backslash$ $F(A)\}$, which is a partition of $A$. Also, $R_{\mathcal{S}_{F}}=\{(F(A), A \backslash F(A))\}$ (in fact, it is not difficult to show that $F$ satisfies WARP). Let $X, Y \in \mathcal{A}$ such that $F(A) \subseteq X$, $Y \subseteq X, Y \nsubseteq F(A), F(A) \nsubseteq Y$, and $Y \cap F(A) \neq \emptyset$. Then $F(X)=F(A)$ but $F(Y)=Y \backslash F(A) \nsubseteq Y \cap F(A)=Y \cap F(X)$. Hence, $F$ does not satisfy WIIA. 


\subsection{Summary}

In this chapter we have established connections between the conditions of WARP, IIA, and WIIA for choice correspondences and their relations with the collections of weak and strong sets. The main results are summarized in Table 1.1 below.

\begin{tabular}{|c|c|c|}
\hline WARP & $\stackrel{\text { Corollary }}{\Longrightarrow} 1.1$ & $F^{2}=F$ \\
\hline \multirow[t]{2}{*}{ WIIA \& $\mathbf{F}^{2}=\mathbf{F}$ v } & $\stackrel{\text { Lemma }}{\Longrightarrow} 1.4$ & WARP \\
\hline & Theorem 1.3 & $\mathcal{S}_{F}$ is a partition, $R_{\mathcal{S}_{F}}$ is complete and acyclic \\
\hline \multirow[t]{2}{*}{ IIA } & Theorem 1.1 & WARP \& PA \\
\hline & Theorem 1.2 & $R_{F}$ is transitive and acyclic \\
\hline
\end{tabular}

Table 1.1: Summary of main results 
20 CHAPTER 1: PREFERENCE AXIOMS FOR MULTI-VALUED CHOICE 


\title{
Chapter 2
}

\section{Solidarity for public goods under single- peaked preferences: Characterizing tar- get set correspondences}

\begin{abstract}
We consider the problem of choosing a set of locations of a public good on the real line $\mathbb{R}$. Similarly to Klaus and Storcken (2002), we ordinally extend the agents' preferences over compact subsets of $\mathbb{R}$, and extend the results of Ching and Thomson (1996), Vohra (1999), and Klaus (2001) to choice correspondences. Specifically, we show that efficiency and either population-monotonicity or one-sided replacement-dominance characterize the class of target set correspondences on the domains of single-peaked preferences and symmetric single-peaked preferences.
\end{abstract}

\subsection{Introduction}

We study the social choice problem where a non-empty and compact set (of points) is chosen on the real line $\mathbb{R}$. We consider this (chosen) set to represent a public good such that each point in the set represents an option for the public good together with its location. We assume that agents have single-peaked preferences, that is, an agent's welfare is strictly increasing up to a certain point, his "peak", and is strictly decreasing beyond this point. Given a non-empty and compact set (of points) that represents the public good's options and their locations, an agent -although in good knowledge of all options and their respective locations- is unable to compute his chance of obtaining the public good at a particular location, e.g., in the case of parking spaces along a street, an agent knows that he will (eventually) find a parking spot somewhere along the street but he does not know where this will be. According to the literature, we should say that the agent needs to make a decision under ignorance (Peterson, 2009, p. 40). We therefore assume that 
agents, when comparing sets, only consider their best (most favorite) point(s) and their worst (least favorite) point(s) in each set. Finally, we assume that the set has adequate capacity to accommodate all agents, that is, all agents have access to the public good but possibly at different locations.

More specifically, we look into the situation where the social planner wishes to make a choice by providing the public good in a way that is efficient, according to the agents' preferences, and that satisfies some notion of solidarity between agents towards changes in circumstances. Loosely speaking, solidarity requires that all agents not responsible for the change should be affected in the same direction. The changes in circumstances we study in this chapter are changes in the agents' population, by considering the property of population-monotonicity, and changes in some agents' preferences, by considering the property of replacementdominance. Population-monotonicity, introduced in the context of bargaining (Thomson, 1983b,a), applies to a model with a variable population of agents and requires that if additional agents join a population, then the agents who were initially present should all be made at least as well off, as they were initially, or they should all be made at most as well off. Replacement-dominance, introduced in the context of quasi-linear binary public decision (Moulin, 1987), applies to a model with a fixed population of agents and requires that if the preferences of an agent change, then the other agents whose preferences remained unchanged should all be made at least as well off, as they were initially, or they should all be made at most as well off.

Further to the parking zone example, already briefly mentioned and further explained in Section 3.2, another example of the described situation could be the following. A social planner drafts an "if-needed" list of candidate locations to build a public hospital according to the agents' preferences. She does so in an effort to narrow down future construction scenarios while at the same time respecting (in an efficient sense) the agents' preferences and adhering to some notion of solidarity, as described above. Then, if at some future time the need to build a hospital materializes, each location in this list is scrutinized and one of them is chosen for the hospital to be built at, with this final verdict assumed unpredictable at the time when the list is drafted.

Many more social choice problems can be phrased as problems of providing a public good by choosing the location of it on the real line $\mathbb{R}$ or an interval of it, or more generally, on a tree network, ${ }^{1}$ when agents have single-peaked preferences. In these types of problems, it is very natural for changes in the population (e.g.,

\footnotetext{
${ }^{1} \mathrm{~A}$ tree network is a connected graph that contains no cycles.
} 
through a change in the birth or migration rate) or changes in the agents' preferences (e.g., through the influence of public media or social networks) to arise. Hence, the properties of population-monotonicity and replacement-dominance have been studied, together or individually, in a variety of contexts. For the special case where the tree network is a closed interval, the problem coincides with the problem of providing a public good by choosing its level when agents have singlepeaked preferences (Moulin, 1980). Apart from the provision of public parking or the provision of a hospital by choosing an "if-needed" list of locations, further examples of providing a public good in one or more locations include the provision of (one or more) schools, parks, or libraries on a tree network that represents an infrastructure, e.g., the network of roads in a neighborhood.

For choice functions that assign a public good on an interval, or on a tree network, the solidarity properties population-monotonicity and replacementdominance, have been considered. Specifically, for the location problem on an interval (on a tree network), it was shown that efficiency and populationmonotonicity characterize the class of "target point functions" on the domain of single-peaked preferences (Thomson, 1993; Ching and Thomson, 1996). ${ }^{2}$ and for constant sets of agents efficiency and replacement-dominance characterize the class of "target point functions" on the domains of single-peaked preferences and symmetric single-peaked preferences (Vohra, 1999). Moreover, it turns out that efficiency and population-monotonicity imply replacement-dominance and also, that the former characterization also holds on the domain of symmetric singlepeaked preferences and on tree networks (Klaus, 2001). In addition, both aforementioned characterizations hold under much looser assumptions on the set of locations (alternatives) and the domain of preferences (Gordon, 2007a). ${ }^{3}$ Finally, if the set of admissible preferences is constrained on attribute-based preference domains, ${ }^{4}$ efficiency and either one of the two solidarity properties are only compatible on discrete trees, where equivalent characterizations are obtained (Gordon, 2015).

For the location problem on an interval, if the property of replacement-

\footnotetext{
${ }^{2}$ Each target point function is determined by its target point: if the target point is efficient, it is chosen; if it is not efficient, the closest efficient point is chosen. Such functions are sometimes called status quo rules or status quo solutions.

${ }^{3}$ The critical assumptions are: (i) the set of alternatives is fixed, (ii) the agents' preferences are defined over all alternatives, and (iii) the domain of preferences is common to all agents.

${ }^{4}$ Given a finite set of alternatives $A$, the non-empty and finite family of subsets $\mathcal{H} \subseteq 2^{A}$ is an attribute space if [for each attribute $H \in \mathcal{H}, H \neq \emptyset$ and the complement $H^{C} \in \mathcal{H}$ ] and [for each pair $x, y \in A$ with $x \neq y$, there exists $H \in \mathcal{H}$ such that $x \in H$ and $y \notin H]$.
} 
dominance is weakened to $\epsilon$-replacement-dominance ${ }^{5}$ the characterization of target point functions still holds for the domain of single-peaked preferences (Harless, 2015a). However, for the location problem on a circle when a constant set of agents exists, no choice function satisfies efficiency and either replacement-dominance or population-monotonicity on the domain of symmetric single-peaked preferences (Gordon, 2007b).

Regarding choice correspondences, the case of providing a public good at exactly two locations, when one or both of the aforementioned solidarity properties are being considered, has been studied under different settings. On the domain of single-peaked preferences and if the agents compare pairs of locations using the max-extension, ${ }^{6}$ the following holds. For an interval in $\mathbb{R}$ and a constant set of agents, the class of choice functions satisfying efficiency and replacementdominance are the "left-peaks choice function" and the "right-peaks choice function"7 (Miyagawa, 2001). However, if this model is extended to trees, then no choice function satisfies efficiency and replacement-dominance on the symmetric single-peaked domain (Umezawa, 2012).

For the problem of providing a public good at exactly two locations on an interval, on the domain of single-peaked preferences and if agents compare pairs of locations using the leximin-extension, ${ }^{8}$ the following two results have been obtained that consider population-monotonicity or replacement-dominance. First, for a constant set of agents the class of choice functions satisfying efficiency, anonymity, and population-monotonicity is the class of "single-plateaued preference choice functions"9 (Ehlers, 2003); and second, in the same setting, the class of choice functions satisfying efficiency and replacement-dominance is the class of "single-peaked preference choice functions" ${ }^{10}$ (Ehlers, 2002).

\footnotetext{
${ }^{5}$ Agents' solidarity is only required if the change in an agent's preferences are below a certain threshold.

${ }^{6}$ Under the max-extension, an agent prefers set $X$ to set $Y$ if and only if he prefers his best point(s) in set $X$ to his best point(s) in set $Y$.

${ }^{7}$ The left (right) peaks choice function chooses the two unique left-most (right-most) peaks.

${ }^{8}$ Under the leximin-extension, in the case of sets containing exactly two points, an agent prefers set $X$ to set $Y$ if and only if he either [prefers his best point(s) in set $X$ to his best point(s) in set $Y$ ] or [he is indifferent between his best point in set $X$ and his best point in set $Y$ and prefers his second best point in set $X$ to his second best point in set $Y$ ].

${ }^{9}$ Each single-plateaued preference choice function is determined by fixed single-plateaued preferences $R$ and plateau $[\underline{r}, \bar{r}]$ : if all the agents' peaks lie outside of $[\underline{r}, \bar{r}]$, then loosely speaking, the best of the agents' peaks and its indifferent point are chosen (according to $R$ ); otherwise, the two locations in the convex hull of the agents' peaks lying closest to $\underline{r}$ and $\bar{r}$ respectively are chosen.

${ }^{10}$ Each single-peaked preference choice function is essentially a single-plateaued preference choice function determined by a fixed single-plateaued preference relation $R$ with the plateau being a point, i.e., $\underline{r}=\bar{r}$.
} 
In the setting of preference aggregation problems, where agents strictly rank a finite set of alternatives and a (not necessarily strict) social ranking over the alternatives must be chosen, the aforementioned solidarity properties have also been studied. It is shown that on the domain of strict rankings, efficiency and population-monotonicity characterize the class of "strict status-quo functions" 11 (Bossert and Sprumont, 2014). Moreover, in this result, population-monotonicity can be substituted with adjacent replacement-dominance. ${ }^{12}$ Furthermore, if the domain is enlarged to also include weak rankings, efficiency and either populationmonotonicity or adjacent replacement-dominance characterize the class of "statusquo functions" ${ }^{13}$ (Harless, 2016).

Finally, in the binary social choice model (i.e., when there are exactly two alternatives to choose from) and if agents can be indifferent between the two alternatives, a choice function satisfies replacement-dominance or populationmonotonicity if and only if it is a "generalized mixed-consensus rule" 14 (Harless, 2015b).

All the above mentioned work analyzes solidarity properties where at each preference profile, either at most two alternatives are chosen or a ranking over the alternatives is chosen. In this chapter we study a class of problems where more than two alternatives might be chosen, which are viewed as locations to provide a public good. This has been considered in a median voter context where the standard choice function setup is extended to choice correspondences since for an even number of agents or voters, a set of median voter locations exists, hence choosing the median implies choosing a set of median points (Klaus and Storcken, 2002). To capture the full spirit of this median voter result, Klaus and Storcken (2002) considered choice correspondences. Our motivation for extending

\footnotetext{
${ }^{11}$ Each strict status-quo function is determined by a strict ranking $R$ over the alternatives and reaches a unique efficient strict ranking as follows: beginning from $R$ it reverses the order of an adjacently ranked pair of alternatives if all agents prefer the reverse to the initial ranking of the pair.

${ }^{12}$ Adjacent replacement-dominance is weaker than replacement-dominance: solidarity is only required when an agent reverses a single pair of adjacently ordered alternatives.

${ }^{13}$ Each status-quo function is determined by a ranking $\bar{R}$ over the alternatives and reaches a unique efficient ranking as follows: beginning from $\bar{R}$ it reverses the order of an adjacently ranked pair of single alternatives if all agents prefer the reverse to the initial ranking of the pair. Moreover, it "creates" order in an indifference class (of alternatives) if all agents prefer the alternative moved up in the order to the one (or more) alternatives moved down. Reversals in the order between a single alternative and an indifference class or between two indifference classes occur in a similar way.

${ }^{14}$ Each generalized mixed-consensus rule chooses for each profile either alternative $a$ or alternative $b$. The only further requirement concerns cases where at least one agent prefers $a$ over $b$ and at least one agent prefers $b$ over $a$; specifically, either $a$ is selected in all such cases or $b$ is selected in all such cases.
} 
choice from one or two locations to a set of locations is that we study situations in which the public good is usually provided through "larger" sets of options, e.g., the assignment of neighborhood parking spots along a street.

On the domain of single-peaked preferences as well as the smaller domain of symmetric single-peaked preferences, we show that the class of choice correspondences satisfying efficiency and either one-sided replacement-dominance ${ }^{15}$ or population-monotonicity, is the class of target set correspondences (Theorems 2.1 and 2.2). Each target set correspondence is determined by a target set $[a, b]$ : if this set is efficient, it is chosen; if it is not efficient, then its largest efficient subset is chosen, if such a subset exists; otherwise, the closest efficient point to the target set is chosen. We also show that efficiency and replacement-dominance characterize the sub-class of target set correspondences where $a=b$, i.e., we obtain the class of target point functions (Corollary 2.3). Hence, we obtain corresponding results with the literature (Thomson, 1993; Ching and Thomson, 1996; Vohra, 1999).

Our results are parallel to the case where the public good is provided via a lottery over locations on an interval, and probabilistic target choice functions are characterized on the basis of efficiency and either one-sided replacementdominance or population-monotonicity (Ehlers and Klaus, 2001).

The chapter proceeds as follows. Section 3.2 explains the model and states some preliminary results. Section 2.3 contains the definition of target set correspondences. Section 2.4 contains the solidarity properties and further preliminary results. Section 2.5 presents characterizations of target set correspondences.

\subsection{The model}

Denote the set of natural numbers by $\mathbb{N}$. There is a grand population of "potential" agents, indexed by $\mathbb{P} \subseteq \mathbb{N}$, where $\mathbb{P}$ contains at least 3 agents. We denote the class of non-empty and finite subsets of $\mathbb{P}$ by $\mathcal{P}$. A set of agents $N \in \mathcal{P}$ is called a population.

Each agent $i \in \mathbb{P}$ is equipped with preferences $R_{i}$, defined on the real line $\mathbb{R}$, that are complete, transitive, and reflexive. As usual, $x R_{i} y$ is interpreted as " $x$ is at least as desirable as $y$ ", $x P_{i} y$ as " $x$ is preferred to $y$ ", and $x I_{i} y$ as " $x$ is indifferent to $y$ ". Moreover, for preferences $R_{i}$ there exists a number $p\left(R_{i}\right) \in \mathbb{R}$, called the peak (level) of agent $i$, with the following property: for each

\footnotetext{
${ }^{15}$ One-sided replacement-dominance is weaker than replacement-dominance: solidarity is not required when the preferences of the agent with the unique smallest peak are changed such that he becomes the agent with the unique largest peak, and vice-versa.
} 
pair $x, y \in \mathbb{R}$ such that either $y<x \leq p\left(R_{i}\right)$, or $y>x \geq p\left(R_{i}\right)$, we have $x P_{i} y$. We call such preferences single-peaked. We denote the domain of all single-peaked preferences on $\mathbb{R}$ by $\mathcal{R}$. Preferences $R_{i}$ are symmetric if for each pair $x, y \in \mathbb{R}$, $\left|x-p\left(R_{i}\right)\right|=\left|y-p\left(R_{i}\right)\right|$ implies $x I_{i} y$. We denote the domain of all symmetric single-peaked preferences on $\mathbb{R}$ by $\mathcal{S}$.

For each population $N \in \mathcal{P}$, we denote the set of (preference) profiles $R=$ $\left(R_{i}\right)_{i \in N}$ where for each $i \in N, R_{i} \in \mathcal{R}$, by $\mathcal{R}^{N}$. Similarly, we denote the set of profiles $R=\left(R_{i}\right)_{i \in N}$, where for each $i \in N, R_{i} \in \mathcal{S}$ by $\mathcal{S}^{N}$. For each pair of populations $N, M \in \mathcal{P}$, with $N \subseteq M$, we denote the restriction $\left(R_{i}\right)_{i \in N} \in \mathcal{R}^{N}$ of profile $R \in \mathcal{R}^{M}$ to population $N$ by $R_{N}$. Given profile $R \in \mathcal{R}^{N}$, for each pair $i, j \in N$ we also use the notation $R_{-i}$ instead of $R_{N \backslash\{i\}}$ and $R_{-i, j}$ instead of $R_{N \backslash\{i, j\}}$.

In the sequel, all notation and definitions refer to single-peaked preferences but also apply to symmetric single-peaked preferences.

Given $N \in \mathcal{P}$ and $R \in \mathcal{R}^{N}$, we denote the (set of) peaks in $R$ as $p(R)=$ $\left\{p\left(R_{i}\right)\right\}_{i \in N}$. Let the smallest peak in $R$ be $\underline{p}(R) \equiv \min \left\{p\left(R_{i}\right)\right\}_{i \in N}$ and the largest peak in $R$ be $\bar{p}(R) \equiv \max \left\{p\left(R_{i}\right)\right\}_{i \in N}$. Let the convex hull of the peaks in $R$ be $\operatorname{Conv}(p(R)) \equiv[\underline{p}(R), \bar{p}(R)]$.

Denote the class of non-empty and compact subsets of $\mathbb{R}$ by $\mathcal{C} .{ }^{16}$ Given a set $X \in \mathcal{C}$, let the minimum (point) of $X$ be $X \equiv \min X$ and the maximum (point) of $X$ be $\bar{X} \equiv \max X$. Given a set $X \in \mathcal{C}$ and preferences $R_{i} \in \mathcal{R}$, let the set of most preferred point(s) or best point(s) of agent $i$ in set $X$ be $b_{X}\left(R_{i}\right) \equiv\{x \in$ $X$ : for each $\left.y \in X, x R_{i} y\right\}$. Similarly, let the set of least preferred point(s) or worst point(s) of agent $i$ in set $X$ be $w_{X}\left(R_{i}\right) \equiv\left\{x \in X\right.$ : for each $\left.y \in X, y R_{i} x\right\}$. Note that by single-peakedness the set $b_{X}\left(R_{i}\right)$ might contain two elements (when agent $i$ 's peak is not included in set $X)$; in this case, agent $i$ is indifferent between these two elements. Similarly, $w_{X}\left(R_{i}\right) \subseteq\{\underline{X}, \bar{X}\}$ and in the case where $w_{X}\left(R_{i}\right)=$ $\{\underline{X}, \bar{X}\}$ and $X \neq \bar{X}$, agent $i$ is again indifferent between these two elements. Hence, with some abuse of notation, we treat sets $b_{X}\left(R_{i}\right)$ and $w_{X}\left(R_{i}\right)$ as if they are points and for each $x \in X$, we write $b_{X}\left(R_{i}\right) R_{i} x R_{i} w_{X}\left(R_{i}\right)$.

We will consider choice correspondences that assign outcomes in $\mathcal{C}$ with the interpretation that any agent "knows the set of possible outcomes..., but has no information about the probabilities of those outcomes or about their likelihood ranking" (Bossert et al., 2000, p. 295). For a survey of criteria and methods for ranking subsets of a set of outcomes under complete uncertainty we refer to

\footnotetext{
${ }^{16}$ As discussed in Remark 2.6, the requirement for sets in $\mathcal{C}$ to be compact is without loss of generality.
} 
Barberà et al. (2004, Section 3).

Before describing the extension of preferences over sets that we use, we first introduce the properties of simple-monotonicity and independence that characterize a small class of preference extensions over sets, albeit for a slightly different model than ours (Bossert et al., 2000, Theorem 1). We first illustrate via two examples why these properties are reasonable to assume in our model. Then, we present the characterization result and finally, we discuss its consequences for our model.

Note that below, we denote preferences defined over $\mathcal{C}$ by $R_{i}^{\mathcal{C}}$ (if $x P_{i} y$, then $\left.\{x\} P_{i}^{\mathcal{C}}\{y\}\right)$.

Simple-monotonicity. Let $x, y \in \mathbb{R}$. If $x P_{i} y$, then $\{x\} P_{i}^{\mathcal{C}}\{x, y\} P_{i}^{\mathcal{C}}\{y\}$.

Independence. Let $X, Y \in \mathcal{C}$ and $z \in \mathbb{R}$ such that $z \notin X \cup Y$. If $X P_{i}^{\mathcal{C}} Y$, then $[X \cup\{z\}] R_{i}^{\mathcal{C}}[Y \cup\{z\}]$.

Both examples that follow pertain to a linear city whose residents own one car each and have single-peaked preferences over where to park.

Example 2.1 (Simple-monotonicity). All public parking is located in two (parking) garages at $x, y \in \mathbb{R}$, with $x \neq y$, that we simply refer to as zone $x$ and $y$. Neither garage's capacity can accommodate all residents but the joint capacity is sufficient. Initially, a one-zone scheme is in place and all residents are assigned to either zone $x$ or zone $y$ : residents assigned to zone $x$ (zone $y$ ) are only allowed to park at garage $x(y)$, which has the capacity to accommodate them. Later, a two-zone scheme is adopted: each resident can use either one of the two garages. Consider a resident $i$ of zone $x$ who prefers $x$ to $y$. Under the one-zone scheme he always parks at $x$, while under the two-zone scheme he sometimes parks at $y$ (whenever $x$ is full). We expect resident $i$ to be worse off under the two-zone scheme, that is, if $x P_{i} y$, then $\{x\} P_{i}^{\mathcal{C}}\{x, y\} P_{i}^{\mathcal{C}}\{y\}$ and simple-monotonicity holds.

Example 2.2 (Independence). Two single-zone street parking schemes, $X \subset \mathbb{R}$ and $Y \subset \mathbb{R}$, are being considered for adoption. Before a final decision is made, and following a small development project on some previously unused land, an extra single parking garage $z \in \mathbb{R}$ becomes available. Now assume that instead of schemes $X$ and $Y$, two new schemes are being considered for adoption, $X \cup\{z\}$ and $Y \cup\{z\}$. Suppose resident $i$ initially prefers $X$ to $Y$. Since space $z$ was unavailable under $X$ and $Y$ and is now available under both $X \cup\{z\}$ and $Y \cup\{z\}$, 
we expect $i$ to find $X \cup\{z\}$ at least as desirable as $Y \cup\{z\}$. That is, if $z \notin X \cup Y$, and $X P_{i}^{\mathcal{C}} Y$, then $[X \cup\{z\}] R_{i}^{\mathcal{C}}[Y \cup\{z\}]$ and independence holds.

The next result shows that if the two aforementioned properties are required, an agent with linear preferences over outcomes ${ }^{17}$ only cares about his best and worst points in each finite set. ${ }^{18}$

Bossert et al. (2000, Theorem 1). If simple-monotonicity and independence are satisfied, then for agent $i$ with linear preferences $R_{i}^{L}$, and each finite set $X \in \mathcal{C}$, $X I_{i}^{\mathcal{C}}\left\{b_{X}\left(R_{i}^{L}\right), w_{X}\left(R_{i}^{L}\right)\right\}$.

In light of this result, two "standard" extensions that could be considered for our model are the $\min$ - $\max ^{19}$ and the $\max -\min ^{20}$ preference extensions, both of which fit our parking example since they are "consistent with the notion of limited rationality which is familiar in the theories of organization and bounded rationality (e.g., March (1988); March and Simon (1958)), and which suggests that, given a complex decision problem, the agent often seeks to simplify the problem by focusing on only a few salient features of the complex situation" (Bossert et al., 2000, pp. 300-301). However, given the problem at hand, we prefer to "not choose sides" by adopting either the "pessimistic" min-max extension or the "optimistic" maxmin extension. Instead, we opt for the best-worst extension of preferences that declares a preference for a set $X$ over a set $Y$ if and only if this preference coincides with the preference of both the min-max extension and the max-min extension. Note however, as we discuss later, that this preference extension is incomplete. Finally, it is straightforward to show that the best-worst extension satisfies, simplemonotonicity and independence, not only when based on linear linear preferences over outcomes but also in our setting of single-peaked preferences over outcomes and sets of alternatives that are not always finite. In the sequel, and with a small abuse of notation, we use the same symbols to denote preferences over points and preferences over sets.

Specifically, under the best-worst extension of preferences over sets, when comparing two sets, an agent only considers his best and his worst point(s) in each

\footnotetext{
${ }^{17} \mathrm{~A}$ linear preference $R^{L}$ is a complete, transitive, reflexive, and antisymmetric (i.e., for each $x, y \in \mathbb{R}, x I^{L} y$ implies $x=y$ ) binary relation. Single-peaked preferences are not antisymmetric.

${ }^{18} \mathrm{~A}$ similar result using a stronger version of independence is shown in Barberà et al. (1984).

${ }^{19} \mathrm{An}$ agent prefers set $X$ to set $Y$ if and only if either [he prefers his worst point(s) in set $X$ to his worst point(s) in set $Y$ ] or [he is indifferent between his worst point(s) in both sets and prefers his best point(s) in set $X$ to his best point(s) in set $Y$ ].

${ }^{20} \mathrm{An}$ agent prefers set $X$ to set $Y$ if and only if either [he prefers his best point(s) in set $X$ to his best point(s) in set $Y$ ] or [he is indifferent between his best point(s) in both sets and prefers his worst point(s) in set $X$ to his worst point(s) in set $Y$ ].
} 
of them. Given two sets $X, Y \in \mathcal{C}$, an agent prefers $X$ to $Y$ if he prefers his best point(s) in $X$ to his best point(s) in $Y$ and his worst point(s) in $X$ to his worst point(s) in $Y$. The following definition also covers three more cases arising if an agent is indifferent between his best or worst point(s) in two sets.

Best-worst extension of preferences to sets. For each agent $i \in \mathbb{P}$ with preferences $R_{i} \in \mathcal{R}$ and each pair of sets $X, Y \in \mathcal{C}$, we have

$$
\begin{aligned}
& X R_{i} Y \text { if and only if }\left\{\begin{array}{c}
b_{X}\left(R_{i}\right) R_{i} b_{Y}\left(R_{i}\right) \\
\text { and } \\
w_{X}\left(R_{i}\right) R_{i} w_{Y}\left(R_{i}\right)
\end{array}\right. \\
& \text { and } \\
& X P_{i} Y \text { if and only if } X R_{i} Y \text { and }\left\{\begin{array}{c}
b_{X}\left(R_{i}\right) P_{i} b_{Y}\left(R_{i}\right) \\
\text { or } \\
w_{X}\left(R_{i}\right) P_{i} w_{Y}\left(R_{i}\right) .
\end{array}\right.
\end{aligned}
$$

This extension of preferences is transitive, i.e., for each triple $X, Y, Z \in \mathcal{C}$, if $X R_{i} Y$ and $Y R_{i} Z$, then $X R_{i} Z$. However, it is not complete: there exist sets $X, Y \in \mathcal{C}$ such that neither $X R_{i} Y$ nor $Y R_{i} X$. To be more precise, we now make the following definition.

Comparability. Sets $X, Y \in \mathcal{C}$ are comparable by agent $i \in \mathbb{P}$ with preferences $R_{i} \in \mathcal{R}$ if and only if $\left[b_{X}\left(R_{i}\right) P_{i} b_{Y}\left(R_{i}\right)\right.$ implies $\left.w_{X}\left(R_{i}\right) R_{i} w_{Y}\left(R_{i}\right)\right]$ and $\left[w_{X}\left(R_{i}\right) P_{i}\right.$ $w_{Y}\left(R_{i}\right)$ implies $\left.b_{X}\left(R_{i}\right) R_{i} b_{Y}\left(R_{i}\right)\right]$.

Regarding the best-worst extension of preferences over sets, we now define Pareto-efficiency, Pareto-dominance, and Pareto-equivalence, henceforth, efficiency, dominance, and equivalence respectively.

Efficiency (of sets). Let $N \in \mathcal{P}$ and $R \in \mathcal{R}^{N}$. Set $X \in \mathcal{C}$ is efficient if and only if there is no set $Y \in \mathcal{C}$ such that for each $i \in N, Y R_{i} X$, and for at least one $j \in N, Y P_{j} X$. We denote the class containing all efficient sets for $R \in \mathcal{R}^{N}$ by $\mathrm{PE}(R)$.

Dominance and equivalence. Let $N \in \mathcal{P}$ and $R \in \mathcal{R}^{N}$. Let pair $X, Y \in \mathcal{C}$ such that for each $i \in N, Y R_{i} X$. If for at least one $j \in N, Y P_{j} X$, then $Y$ dominates $X$, otherwise $Y$ and $X$ are equivalent.

We now proceed to characterize efficient sets. 
Proposition 2.1 (Efficient sets). For each $N \in \mathcal{P}$ and each $R \in \mathcal{R}^{N}$, a set $X \in \mathcal{C}$ is efficient if and only if the following two conditions hold.

(i) $X$ is a subset of the convex hull of the agents' peaks. That is,

$$
X \subseteq \operatorname{Conv}(p(R))
$$

(ii) All of the agents' peaks that lie in the convex hull of $X$ are included in $X$. That is,

$$
\operatorname{Conv}(X) \cap p(R) \subseteq X
$$

We prove Proposition 2.1 in Appendix 2.A and illustrate it in Figure 2.1.

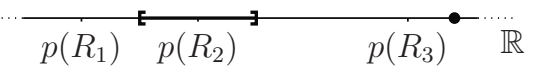

(a)

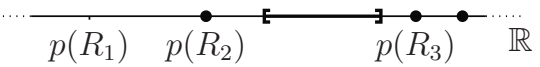

(c)

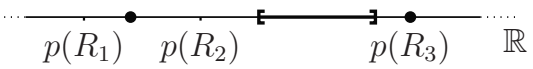

(b)

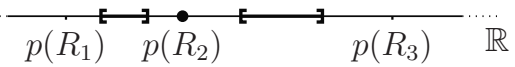

(d)

Figure 2.1: Let $N=\{1,2,3\}$ with $R \in \mathcal{R}^{N}$ and $p(R)=\left\{p\left(R_{1}\right), p\left(R_{2}\right), p\left(R_{3}\right)\right\}$. Sets under consideration are shown in bold. The set in (a) satisfies neither (i) nor (ii). The set in (b) satisfies (i) but not (ii). The set in (c) does not satisfy (i) but it satisfies (ii). The set in (d) satisfies both (i) and (ii), hence it is efficient.

When considering convex sets, the characterization in Proposition 2.1 simplifies.

Remark 2.1 (Efficient convex sets). For each $N \in \mathcal{P}$, each $R \in \mathcal{R}^{N}$, and each convex set $X=\operatorname{Conv}(X) \in \mathcal{C}, X \in \mathrm{PE}(R)$ if and only if $X \subseteq \operatorname{Conv}(p(R))$.

Further consequences of Proposition 2.1 are Corollaries 2.1 and 2.2. Essentially, Corollary 2.1 states that given a population $M$ with profile $R$, if $X \in \mathcal{C}$ is efficient, then it is also efficient for each population $N \subsetneq M$ such that the convex hull of population $N$ 's peaks at profile $R_{N}$, and that of population $M$ 's peaks at profile $R$, are the same.

Corollary 2.1. Let $M \in \mathcal{P}, R \in \mathcal{R}^{M}$, and $X \in \operatorname{PE}(R)$. Then, for each $N \in \mathcal{P}$ such that $N \subsetneq M$ and $\operatorname{Conv}\left(p\left(R_{N}\right)\right)=\operatorname{Conv}(p(R)), X \in \operatorname{PE}\left(R_{N}\right)$. 
Proof. Let $N, M \in \mathcal{P}$ be such that $N \subsetneq M, R \in \mathcal{R}^{M}$, and $X \in \mathrm{PE}(R)$. By Proposition 2.1 (i), $X \subseteq \operatorname{Conv}(p(R))$. Since, $\operatorname{Conv}(p(R))=\operatorname{Conv}\left(p\left(R_{N}\right)\right), X \subseteq$ $\operatorname{Conv}\left(p\left(R_{N}\right)\right)$. By Proposition 2.1 (ii), $\operatorname{Conv}(X) \cap p(R) \subseteq X$. Since, $p\left(R_{N}\right) \subsetneq p(R)$, $\operatorname{Conv}(X) \cap p\left(R_{N}\right) \subseteq X$. By Proposition 2.1, $X \in \mathrm{PE}\left(R_{N}\right)$.

Corollary 2.2 provides some consequences for efficient and equivalent sets.

Corollary 2.2. Let $N \in \mathcal{P}, R \in \mathcal{R}^{N}$, and $X \in \operatorname{PE}(R)$. Then, $\operatorname{Conv}(X)$ is equivalent to $X$. Moreover, if $Y$ is equivalent to $X$, then $\operatorname{Conv}(Y)=\operatorname{Conv}(X)$.

We prove Corollary 2.2 in Appendix 2.A. Moreover, to simplify notation, in the sequel we always represent any efficient set by its convex hull.

\subsection{Choice correspondences}

A choice correspondence $F$ assigns to each $N \in \mathcal{P}$ and each $R \in \mathcal{R}^{N}$ a set $F(R) \in$ $\mathcal{C}$, i.e., $F: \bigcup_{N \in \mathcal{P}} \mathcal{R}^{N} \rightarrow \mathcal{C}$. We denote the family of choice correspondences $F$ by $\mathcal{F}$.

In the sequel, when the properties of replacement-dominance and one-sided replacement-dominance (defined in Section 2.4) are considered, the population of agents does not change. For this reason, we introduce fixed-population choice correspondences, henceforth fp-choice correspondences.

Given $N \in \mathcal{P}$, an $f p$-choice correspondence $F$ for $N$ assigns to each $R \in \mathcal{R}^{N}$ a set $F(R) \in \mathcal{C}$, i.e., $F: \mathcal{R}^{N} \rightarrow \mathcal{C}$. Let $\mathcal{F}^{N}$ denote the family of fp-choice correspondences for $N$. A choice correspondence is a collection of fp-choice correspondences indexed by $N \in \mathcal{P}$.

Remark 2.2 (Choice functions). Given population $N \in \mathcal{P}$, if an fp-choice correspondence for $N$ assigns to each $R \in \mathcal{R}^{N}$ a set consisting of a single point, it is essentially an fp-choice function. Similarly, if a choice correspondence assigns to each $N \in \mathcal{P}$ and each $R \in \mathcal{R}^{N}$ a set consisting of a single point, it is essentially a choice function.

We now proceed to our efficiency notion for fp-choice correspondences and choice correspondences.

Efficiency (of choice correspondences).

(a) Let $N \in \mathcal{P}$ and $F \in \mathcal{F}^{N}$ be an fp-choice correspondence. For each $R \in$ $\mathcal{R}^{N}, F(R) \in \operatorname{PE}(R)$. 
(b) Let choice correspondence $F \in \mathcal{F}$. For each $N \in \mathcal{P}$ and each $R \in \mathcal{R}^{N}$, $F(R) \in \mathrm{PE}(R)$.

The following classes of "target (choice) correspondences" and "fp-target (choice) correspondences" play an important role in the sequel.

Any fp-target point correspondence is determined by its fixed population and its target point. Similarly, any target point correspondence is determined by its target point. In both cases: if the target point is efficient, then it is chosen. If the target point is not efficient, then the (unique) closest efficient point to it is chosen.

Target point correspondences. Let $a \in \mathbb{R} \cup\{-\infty, \infty\}$. We define:

(a) for population $N \in \mathcal{P}$, the fp-target point correspondence with target $a, f^{a} \in$ $\mathcal{F}^{N}$, such that for each $R \in \mathcal{R}^{N}$,

$$
f^{a}(R)= \begin{cases}\{\underline{p}(R)\} & \text { if } a<\underline{p}(R) \\ \{\bar{p}(R)\} & \text { if } a>\bar{p}(R) \\ \{a\} & \text { otherwise. }\end{cases}
$$

A (fp-)target point correspondence $f^{a}$ is essentially a ( fp-)target point function. $^{21}$

Any fp-target set correspondence is determined by its population and its nonempty, closed, and convex target set. Similarly, any target set correspondence is determined by its non-empty, closed, and convex target set. In both cases: if the target set is efficient, it is chosen. If the target set is not efficient, the (unique) maximal efficient subset of the target set is chosen, if one exists; otherwise, the (unique) closest efficient point to the target set is chosen.

Target set correspondences. Let $[a, b] \subseteq \mathbb{R} \cup\{-\infty, \infty\}$. We define:

(a) for population $N \in \mathcal{P}$, the $f p$ target set correspondence with target $[a, b], F^{a, b} \in \mathcal{F}^{N}$, such that for each $R \in \mathcal{R}^{N}$,

(b) the target set correspondence with target set $[a, b], F^{a, b} \in \mathcal{F}$, such that for each $N \in \mathcal{P}$ and each $R \in \mathcal{R}^{N}$,

$$
F^{a, b}(R)= \begin{cases}\{\underline{p}(R)\} & \text { if } b<\underline{p}(R) \\ \{\bar{p}(R)\} & \text { if } a>\bar{p}(R) \\ {[a, b] \cap \operatorname{Conv}(p(R))} & \text { otherwise. }\end{cases}
$$

\footnotetext{
${ }^{21}$ The difference is that a (fp-)target point correspondence $f^{a}$ only assigns singleton sets while the corresponding (fp-)target point function assigns the points in these sets.
} 
Each target set correspondence is a set of fp-target set correspondences, one for each $N \in \mathcal{P}$, where the target set is constant and independent of the population. Also, each (fp-)target set correspondence with a target set $[a, b] \subseteq \mathbb{R} \cup\{-\infty, \infty\}$ such that $a=b$, is a (fp-)target point correspondence.

By Proposition 2.1, it follows that each (fp-)target set correspondence satisfies efficiency.

We illustrate the concept of an fp-target set correspondence in Figure 2.2. Since each target set correspondence is a collection of fp-target set correspondences indexed by $N \in \mathcal{P}$, a similar example for target set correspondences can be easily obtained if in Figure 2.2 we allow for the population to change.

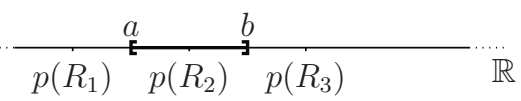

(a)

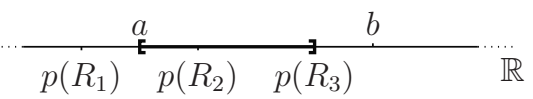

(b)

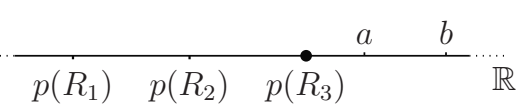

(c)

Figure 2.2: Let $N=\{1,2,3\}$ with $R \in \mathcal{R}^{N}$ and $p(R)=\left\{p\left(R_{1}\right), p\left(R_{2}\right), p\left(R_{3}\right)\right\}$. Let $F^{a, b} \in \mathcal{F}^{N}$. The chosen sets in each case are shown in bold. The target set in (a) is efficient and is chosen. The target set in (b) is not efficient but the maximal efficient subset exists and it is chosen. The target set in (c) is not efficient and no maximal efficient subset exists; hence the closest efficient point is chosen.

\section{Remark 2.3 (Properties of fp-choice correspondences extend to choice} correspondences). In Section 2.4, we introduce properties of fp-choice correspondences. Since a choice correspondence is a collection of fp-choice correspondences, these properties easily extend to choice correspondences.

\subsection{Properties of choice correspondences}

In the sequel, all properties and results refer to single-peaked preferences but also apply to symmetric single-peaked preferences.

We consider two solidarity properties of choice correspondences. The first solidarity property, expresses the solidarity among agents against changes in the population (Thomson, 1983b,a): if agents are added to the population, the agents 
initially present should all be made at least as well off or they should all be made at most as well off by this change.

Population-monotonicity. Let $F \in \mathcal{F}$ be a choice correspondence. For each pair $N, M \in \mathcal{P}$ such that $N \subseteq M$ and each $R \in \mathcal{R}^{M}$ the following holds:

for each $i \in N, F\left(R_{N}\right) R_{i} F(R)$ or for each $i \in N, F(R) R_{i} F\left(R_{N}\right)$.

Population-monotonicity implies that the chosen sets, before and after the change in population, are comparable, and in the same way in terms of their welfare, by all agents present before and after this change.

The next lemma states that if a choice correspondence satisfies efficiency and population-monotonicity, then if agents are added to the population, all agents who were initially present are at most as well off.

Lemma 2.1 (Efficiency and population-monotonicity). Let choice correspondence $F \in \mathcal{F}$ satisfy efficiency and population-monotonicity. Then, for each pair $N, M \in \mathcal{P}$ such that $N \subseteq M$, each $R \in \mathcal{R}^{M}$, and each $i \in N, F\left(R_{N}\right) R_{i} F(R)$. In particular, if $\operatorname{Conv}\left(p\left(R_{N}\right)\right)=\operatorname{Conv}(p(R))$, then $F\left(R_{N}\right)=F(R)$.

Proof. Let choice correspondence $F \in \mathcal{F}$ satisfy efficiency and populationmonotonicity. Let $N, M \in \mathcal{P}$ be such that $N \subseteq M$. Let $R \in \mathcal{R}^{M}$.

By efficiency, $F(R) \in \mathrm{PE}(R)$ and $F\left(R_{N}\right) \in \mathrm{PE}\left(R_{N}\right)$. By populationmonotonicity, for each $i \in N, F(R) R_{i} F\left(R_{N}\right)$ or for each $i \in N, F\left(R_{N}\right) R_{i} F(R)$. If for each $i \in N, F(R) R_{i} F\left(R_{N}\right)$ and since $F\left(R_{N}\right) \in \operatorname{PE}\left(R_{N}\right)$, then for each $i \in N, F\left(R_{N}\right) I_{i} F(R)$. Therefore, for each $i \in N, F\left(R_{N}\right) R_{i} F(R)$.

In particular, if $\operatorname{Conv}\left(p\left(R_{N}\right)\right)=\operatorname{Conv}(p(R))$, then by $F(R) \in \mathrm{PE}(R)$ and Corollary 2.1, $F(R) \in \mathrm{PE}\left(R_{N}\right)$. Since for each $i \in N, F\left(R_{N}\right) R_{i} F(R)$, and moreover $\left[F(R) \in \mathrm{PE}\left(R_{N}\right)\right.$ and $\left.F\left(R_{N}\right) \in \mathrm{PE}\left(R_{N}\right)\right]$, then for each $i \in N, F\left(R_{N}\right) I_{i} F(R)$. By Corollary 2.2, $\operatorname{Conv}\left(F\left(R_{N}\right)\right)=\operatorname{Conv}(F(R))$, and since we always represent any efficient set by its convex hull, $F\left(R_{N}\right)=F(R)$.

Proposition $2.2\left(\boldsymbol{F}^{a, b}\right.$ is population-monotonic). Each target set correspondence satisfies population-monotonicity.

Proof. Let $F^{a, b} \in \mathcal{F}$ be a target set correspondence. Let $N \in \mathcal{P}$ be such that $|N| \geq 2$ and $R \in \mathcal{R}^{N}$. We prove population-monotonicity of $F^{a, b}$ by showing that if $j \in N$ leaves all remaining agents end up at least as well off, i.e., for each $i \in N \backslash\{j\}, F^{a, b}\left(R_{-j}\right) R_{i} F^{a, b}(R)$. 
Case 1. $\operatorname{Conv}\left(p\left(R_{-j}\right)\right)=\operatorname{Conv}(p(R))$. Then, the chosen set remains the same, $F^{a, b}\left(R_{-j}\right)=F^{a, b}(R)$.

Case 2. $\operatorname{Conv}\left(p\left(R_{-j}\right)\right) \neq \operatorname{Conv}(p(R))$. Then, $j$ has either the unique smallest peak at $R$ or the unique largest peak at $R$. By symmetry of arguments, assume that $j$ has the unique smallest peak at $R, p\left(R_{j}\right)=\underline{p}(R)$. Then, $\underline{p}(R)<\underline{p}\left(R_{-j}\right)$. There are 3 possibilities.

(i) $a, b<\underline{p}\left(R_{-j}\right)$. Then, $F^{a, b}\left(R_{-j}\right)=\underline{p}\left(R_{-j}\right)$. Furthermore, if $b \leq p\left(R_{j}\right)$, then $F^{a, b}(R)=p\left(R_{j}\right)$; if $a \leq p\left(R_{j}\right)$ and $b>p\left(R_{j}\right)$, then $F^{a, b}(R)=\left[p\left(R_{j}\right), b\right]$; and if $a>p\left(R_{j}\right)$, then $F^{a, b}(R)=[a, b]$. Hence, for each $i \in N \backslash\{j\}, b_{F^{a, b}\left(R_{-j}\right)}\left(R_{i}\right)=$ $w_{F^{a, b}\left(R_{-j}\right)}\left(R_{i}\right)=\underline{p}\left(R_{-j}\right), b_{F^{a, b}(R)}\left(R_{i}\right) \in\left\{p\left(R_{j}\right), b\right\}$, and $w_{F^{a, b}(R)}\left(R_{i}\right) \in\left\{p\left(R_{j}\right), a\right\}$. Thus, for each $i \in N \backslash\{j\}, b_{F^{a, b}(R)}\left(R_{i}\right)<b_{F^{a, b}\left(R_{-j}\right)}\left(R_{i}\right) \leq p\left(R_{i}\right)$ and $w_{F^{a, b}(R)}\left(R_{i}\right)<$ $w_{F^{a, b}\left(R_{-j}\right)}\left(R_{i}\right) \leq p\left(R_{i}\right)$. By single-peakedness, for each $i \in N \backslash\{j\}$, the best and worst points are improved. Hence, $F^{a, b}\left(R_{-j}\right) P_{i} F^{a, b}(R)$.

(ii) $a<\underline{p}\left(R_{-j}\right)$ and $b \geq \underline{p}\left(R_{-j}\right)$. Then, $\underline{F}^{a, b}(R)<\underline{F}^{a, b}\left(R_{-j}\right)=\underline{p}\left(R_{-j}\right)$ and $\bar{F}^{a, b}(R)=\bar{F}^{a, b}\left(R_{-j}\right)$. Thus, for each $i \in N \backslash\{j\}, \underline{F}^{a, b}(R)<\underline{F}^{a, b}\left(R_{-j}\right) \leq p\left(R_{i}\right)$. If $\bar{F}^{a, b}\left(R_{-j}\right)<p\left(R_{i}\right)$, then $b_{F^{a, b}(R)}\left(R_{i}\right)=b_{F^{a, b}\left(R_{-j}\right)}\left(R_{i}\right)<p\left(R_{i}\right)$ and $w_{F^{a, b}(R)}\left(R_{i}\right)<$ $w_{F^{a, b}\left(R_{-j}\right)}\left(R_{i}\right)<p\left(R_{i}\right)$. Hence, by single-peakedness, $i$ 's best point is at least as desirable and his worst point is improved. If $\bar{F}^{a, b}\left(R_{-j}\right) \geq p\left(R_{i}\right)$, then $b_{F^{a, b}(R)}\left(R_{i}\right)=$ $b_{F^{a, b}\left(R_{-j}\right)}\left(R_{i}\right)=p\left(R_{i}\right)$ and $w_{F^{a, b}\left(R_{-j}\right)}\left(R_{i}\right) \in F^{a, b}\left(R_{-j}\right) \subseteq F^{a, b}(R)$. Thus, $i$ 's best and worst points are at least as desirable. Hence, for each $i \in N \backslash\{j\}, F^{a, b}\left(R_{-j}\right) R_{i}$ $F^{a, b}(R)$.

(iii) $a, b \geq \underline{p}\left(R_{-j}\right)$. Then, the chosen set remains the same, $F^{a, b}\left(R_{-j}\right)=$ $F^{a, b}(R)$.

The second solidarity property we consider expresses the solidarity among agents against changes in preferences (Moulin, 1987): if the preferences of an agent change, then the other agents should all be made at least as well off or they should all be made at most as well off. We formulate this requirement for fpchoice correspondences but as discussed in Remark 2.3, it easily extends to choice correspondences.

Replacement-dominance. Let $N \in \mathcal{P}$ and $F \in \mathcal{F}^{N}$ be an fp-choice correspondence. For each $j \in N$, and each pair $R, \bar{R} \in \mathcal{R}^{N}$ such that $R_{-j}=\bar{R}_{-j}$ the following holds:

for each $i \in N \backslash\{j\}, F(R) R_{i} F(\bar{R})$ or for each $i \in N \backslash\{j\}, F(\bar{R}) R_{i} F(R)$.

Replacement-dominance implies that the chosen sets, before and after the 
change in preferences of some agent, are comparable by all other agents.

Note that for a population of one or two agents, replacement-dominance imposes no restriction on fp-choice correspondences. Hence, for each fixed population with one or two agents, each fp-target set correspondence satisfies replacementdominance. However, if the fixed population contains at least three agents, then the target set must equal a point.

Proposition $2.3\left(\boldsymbol{F}^{[a, b]}\right.$ is replacement-dominant $\left.\Leftrightarrow \boldsymbol{a}=\boldsymbol{b}\right)$. If a population consists of at least 3 agents, then an associated fp-target set correspondence satisfies replacement-dominance if and only if it is an fp-target point correspondence.

Proof. Let $N \in \mathcal{P}$ be such that $|N| \geq 3$ and $F^{a, b} \in \mathcal{F}^{N}$ be an fp-target set correspondence.

First, if $a=b$, we prove replacement-dominance of $f^{a}\left(F^{a, b}, a=b\right)$ by showing that for each pair $R, \bar{R} \in \mathcal{R}^{N}$ such that $\bar{R} \in \mathcal{R}^{N}$ and $R_{-j}=\bar{R}_{-j}$, [for each $i \in N \backslash\{j\}, f^{a}(R) R_{i} f^{a}(\bar{R})$ ] or [for each $i \in N \backslash\{j\}, f^{a}(\bar{R}) R_{i} f^{a}(R)$ ].

Case 1. $\operatorname{Conv}(p(\bar{R}))=\operatorname{Conv}(p(R))$. Then, the set (point) chosen remains the same, $f^{a}(\bar{R})=f^{a}(R)$.

Case 2.1. $\operatorname{Conv}(p(\bar{R})) \subsetneq \operatorname{Conv}(p(R))$. Then, $j$ has either the unique smallest peak at $R$ or the unique largest peak at $R$. By symmetry of arguments, assume that $p\left(R_{j}\right)=\underline{p}(R)$. Then, $\underline{p}(R)<\underline{p}(\bar{R}) \leq \bar{p}(R)=\bar{p}(\bar{R})$. There are 2 possibilities.

(i) $a<\underline{p}(\bar{R})$. Then, $f^{a}(\bar{R})=\{\underline{p}(\bar{R})\}$. Furthermore, if $a \leq \underline{p}(R)$, then $f^{a}(R)=\{\underline{p}(R)\}$ and if $a>\underline{p}(R)$, then $f^{a}(R)=\{a\}$. Hence, for each $i \in N \backslash\{j\}$, $f^{a}(R)<f^{a}(\bar{R}) \leq p\left(\bar{R}_{i}\right)$. Hence, by single-peakedness, for each $i \in N \backslash\{j\}$, $f^{a}(\bar{R}) P_{i} f^{a}(R)$.

(ii) $a \geq \underline{p}(\bar{R})$. Then, the set (point) chosen remains the same, $f^{a}(\bar{R})=f^{a}(R)$.

Case 2.2. $\operatorname{Conv}(p(\bar{R})) \supsetneq \operatorname{Conv}(p(R))$. Then, by Case 2.1 (with the roles of $R$ and $\bar{R}$ reversed), for each $i \in N \backslash\{j\}, f^{a}(R) R_{i} f^{a}(\bar{R})$.

Case 3. $\operatorname{Conv}(p(\bar{R})) \nsubseteq \operatorname{Conv}(p(R))$ and $\operatorname{Conv}(p(\bar{R})) \nsupseteq \operatorname{Conv}(p(R))$. Then, $j$ has either [the unique smallest peak at $R$ and the unique largest peak at $\bar{R}$ ] or [the unique largest peak at $R$ and the unique smallest peak at $\bar{R}]$. By symmetry of arguments, assume that $p\left(R_{j}\right)=\underline{p}(R)$ and $p\left(\bar{R}_{j}\right)=\bar{p}(\bar{R})$. Then, $\underline{p}(R)<\underline{p}(\bar{R}) \leq$ $\bar{p}(R)<\bar{p}(\bar{R})$. There are 3 possibilities.

(i) $a<\underline{p}(\bar{R})$. Then, as shown in Case 2.1, for each $i \in N \backslash\{j\}, f^{a}(\bar{R}) P_{i} f^{a}(R)$.

(ii) $\underline{p}(\bar{R}) \leq a \leq \bar{p}(R)$. Then, the set (point) chosen remains the same, $f^{a}(\bar{R})=f^{a}(R)$

(iii) $a>\bar{p}(R)$. Then, $f^{a}(R)=\{\bar{p}(R)\}$. Furthermore, if $a \geq \bar{p}(\bar{R})$, then $f^{a}(\bar{R})=\{\bar{p}(\bar{R})\}$ and if $a<\bar{p}(\bar{R})$, then $f^{a}(\bar{R})=\{a\}$. Hence, for each $i \in N \backslash\{j\}$, 
$p\left(\bar{R}_{i}\right) \leq f^{a}(R)<f^{a}(\bar{R})$. Hence, by single-peakedness, for each $i \in N \backslash\{j\}$, $f^{a}(R) P_{i} f^{a}(\bar{R})$.

Second, we prove that if $a<b$, then $F^{a, b}$ does not satisfy replacementdominance. Without loss of generality, assume that $1,2,3 \in N$.

If $a=-\infty$, let $\bar{a} \in \mathbb{R}$ be such that $\bar{a}<b$, otherwise, let $\bar{a}=a$. If $b=\infty$, then let $\bar{b} \in \mathbb{R}$ be such that $\bar{b}>\bar{a}$, otherwise, let $\bar{b}=b$. Hence, $[\bar{a}, \bar{b}] \subseteq[a, b]$. We divide the interval $[\bar{a}, \bar{b}]$ into three equal parts and use the four points $a_{1}=\bar{a}$, $a_{2}=\left(\bar{a}+\frac{1}{3}(\bar{b}-\bar{a})\right), a_{3}=\left(\bar{a}+\frac{2}{3}(\bar{b}-\bar{a})\right)$, and $a_{4}=\bar{b}$ to construct (symmetric) profiles $R, \bar{R} \in \mathcal{S}^{N}$ such that $p\left(R_{1}\right)=a_{1}, p\left(R_{2}\right)=p\left(\bar{R}_{2}\right)=a_{2}, p\left(R_{3}\right)=p\left(\bar{R}_{3}\right)=a_{3}$, $p\left(\bar{R}_{1}\right)=a_{4}$, and for each $i \in N \backslash\{1,2,3\}, p\left(R_{i}\right)=p\left(\bar{R}_{i}\right)=a_{2}$. Note that $R_{-1}=\bar{R}_{-1}$.

By the definition of $F^{a, b}$, we have $F^{a, b}(R)=\left[a_{1}, a_{3}\right]$ and $F^{a, b}(\bar{R})=\left[a_{2}, a_{4}\right]$. Under both $R$ and $\bar{R}$, the best points of agents 2 and 3 remain the same, $b_{F^{a, b}(R)}\left(R_{2}\right)=b_{F^{a, b}(\bar{R})}\left(R_{2}\right)=p\left(R_{2}\right)$ and $b_{F^{a, b}(R)}\left(R_{3}\right)=b_{F^{a, b}(\bar{R})}\left(R_{3}\right)=p\left(R_{3}\right)$. However, the worst points of agent 2 and 3 change as follows. For agent 2, $w_{F^{a, b}(R)}\left(R_{2}\right)=\left\{a_{1}, a_{3}\right\}$ and $w_{F^{a, b}(\bar{R})}\left(R_{1}\right)=\left\{a_{4}\right\}$. Since $p\left(R_{2}\right)=a_{2}<a_{3}<a_{4}$, single-peakedness implies $F^{a, b}(R) P_{2} F^{a, b}(\bar{R})$. For agent $3, w_{F^{a, b}(R)}\left(R_{3}\right)=\left\{a_{1}\right\}$ and $w_{F^{a, b}(\bar{R})}\left(R_{3}\right)=\left\{a_{2}, a_{4}\right\}$. Since $a_{1}<a_{2}<a_{3}=p\left(R_{3}\right)$, single-peakedness implies $F^{a, b}(\bar{R}) P_{3} F^{a, b}(R)$. This contradicts replacement-dominance.

We next introduce a property weaker than replacement-dominance in the sense that it does not require solidarity when the preferences of the agent with the unique smallest peak are changed such that he becomes the agent with the unique largest peak, or vice-versa; in other words, following a change in preferences of some agent, solidarity is required only if one side of the convex hull of the agents' peaks has remained the same. We formulate this requirement for fp-choice correspondences but as discussed in Remark 2.3, it easily extends to choice correspondences.

One-sided replacement-dominance. Let $N \in \mathcal{P}$ and $F \in \mathcal{F}^{N}$ be an fp-choice correspondence. For each $j \in N$ and each pair $R, \bar{R} \in \mathcal{R}^{N}$ such that $R_{-j}=\bar{R}_{-j}$ and $\operatorname{Conv}(p(R)) \subseteq \operatorname{Conv}(p(\bar{R}))$ or $\operatorname{Conv}(p(R)) \supseteq \operatorname{Conv}(p(\bar{R}))$ the following holds:

for each $i \in N \backslash\{j\}, F(R) R_{i} F(\bar{R})$ or for each $i \in N \backslash\{j\}, F(\bar{R}) R_{i} F(R)$.

One-sided replacement-dominance implies that the chosen sets, before and after the change in preferences of some agent, are comparable, and in the same way in terms of their welfare, by all other agents. Moreover, replacement-dominance implies one-sided replacement-dominance. 
The next lemma states that given a population of at least three agents and an associated fp-choice correspondence satisfying efficiency and one-sided replacement-dominance, if the preferences of an agent change in such a way that the new set of peaks is a subset of the initial one, all other agents end up at least as well off.

Lemma 2.2 (Efficiency and one-sided replacement-dominance). Let $N \in$ $\mathcal{P}$ be such that $|N| \geq 3$ and fp-choice correspondence $F \in \mathcal{F}^{N}$ satisfy efficiency and one-sided replacement-dominance. Then, for each $j \in N$, each pair $R, \bar{R} \in \mathcal{R}^{N}$ such that $\left[R_{-j}=\bar{R}_{-j}\right.$ and $\left.\operatorname{Conv}(p(\bar{R})) \subseteq \operatorname{Conv}(p(R))\right]$, and each $i \in N \backslash\{j\}$, $F(\bar{R}) R_{i} F(R)$. In particular, if $\operatorname{Conv}(p(\bar{R}))=\operatorname{Conv}(p(R))$, then $F(\bar{R})=F(R)$.

We prove Lemma 2.2 in Appendix 2.B. Moreover, recall that for a population $N \in \mathcal{P}$ with one or two agents (one-sided) replacement-dominance imposes no restriction on an associated fp-choice correspondence. The following example illustrates why Lemma 2.2 does not hold for a population of two agents and an associated fp-choice correspondence.

Example 2.3. Let $N \in \mathcal{P}$ be such that $N=\{1,2\}$ and $F \in \mathcal{F}^{N}$ be an fp-choice correspondence such that

$$
F(R)= \begin{cases}p\left(R_{2}\right) & \text { if } p\left(R_{2}\right)=1 \\ p\left(R_{1}\right) & \text { otherwise }\end{cases}
$$

Hence, $F$ satisfies efficiency, and since $|N|=2$, it trivially satisfies (onesided) replacement-dominance. Let $R, \bar{R} \in \mathcal{R}^{N}$ be such that $p\left(R_{1}\right)=p\left(\bar{R}_{1}\right)=0$, $p\left(R_{2}\right)=2$, and $p\left(\bar{R}_{2}\right)=1$. Hence, $\operatorname{Conv}(p(\bar{R})) \subsetneq \operatorname{Conv}(p(R))$. It follows, that $F(R)=0$ and $F(\bar{R})=1$. Hence, agent 1's peak $p\left(R_{1}\right)=F(R)<F(\bar{R})$. By single-peakedness, $F(R) P_{1} F(\bar{R})$.

Proposition 2.4 ( $\boldsymbol{F}^{a, b}$ is one-sided replacement-dominant). Each fp-target set correspondence satisfies one-sided replacement-dominance.

Proof. Let $N \in \mathcal{P}$ and $F^{a, b} \in \mathcal{F}^{N}$ be an fp-target set correspondence. Since for $|N| \leq 2$, (one-sided) replacement-dominance imposes no restriction on fp-choice correspondence $F^{a, b}$, fix $|N| \geq 3$.

We prove that $F^{a, b}$ satisfies one-sided replacement-dominance, i.e., we show that for each $R, \bar{R} \in \mathcal{R}^{N}$ such that $R_{-j}=\bar{R}_{-j}$ and $\operatorname{Conv}(p(R)) \subseteq \operatorname{Conv}(p(\bar{R}))$ or $\operatorname{Conv}(p(\bar{R})) \subseteq \operatorname{Conv}(p(R))$, the following holds. For each $i \in N \backslash\{j\}, F^{a, b}(R) R_{i}$ $F^{a, b}(\bar{R})$ or for each $i \in N \backslash\{j\}, F^{a, b}(\bar{R}) R_{i} F^{a, b}(R)$. 
Case 1. $\operatorname{Conv}(p(\bar{R}))=\operatorname{Conv}(p(R))$. Then, the chosen set remains the same, $F^{a, b}(\bar{R})=F^{a, b}(R)$.

Case 2.1. $\operatorname{Conv}(p(\bar{R})) \subsetneq \operatorname{Conv}(p(R))$. Then, $j$ has either the unique smallest peak at $R$ or the unique largest peak at $R$. By symmetry of arguments, assume that $j$ has the unique smallest peak at $R, p\left(R_{j}\right)=\underline{p}(R)$. Then, $\underline{p}(R)<\underline{p}(\bar{R}) \leq$ $\bar{p}(R)=\bar{p}(\bar{R})$. There are 3 possibilities.

(i) $a, b<\underline{p}(\bar{R})$. Then $F^{a, b}(\bar{R})=\underline{p}(\bar{R})$. Furthermore, if $a, b \leq \underline{p}(R)$, then $F^{a, b}(R)=\underline{p}(R)$; if $a \leq \underline{p}(R)$ and $b>\underline{p}(R)$, then $F^{a, b}(R)=[\underline{p}(R), b]$; and if $a, b>\underline{p}(R)$, then $F^{a, b}(R)=[a, b]$. Hence, for each $i \in N \backslash\{j\}, b_{F^{a, b}(\bar{R})}\left(R_{i}\right)=$ $w_{F^{a, b}(\bar{R})}\left(R_{i}\right)=\{\underline{p}(\bar{R})\}, b_{F^{a, b}(R)}\left(R_{i}\right) \in\{\underline{p}(R), b\}$, and $w_{F^{a, b}(R)}\left(R_{i}\right) \in\{\underline{p}(R), a\}$. Thus, for each $i \in N \backslash\{j\}, b_{F^{a, b}(R)}\left(R_{i}\right)<b_{F^{a, b}(\bar{R})}\left(R_{i}\right) \leq p\left(R_{i}\right)$ and $w_{F^{a, b}(R)}\left(R_{i}\right)<$ $w_{F^{a, b}(\bar{R})}\left(R_{i}\right) \leq p\left(R_{i}\right)$. By single-peakedness, for each $i \in N \backslash\{j\}$, best and worst points improve. Hence, $F^{a, b}(\bar{R}) P_{i} F^{a, b}(R)$.

(ii) $a<p(\bar{R})$ and $b \geq p(\bar{R})$. Then, for the minima $\underline{F}^{a, b}(R)$ and $\underline{F}^{a, b}(\bar{R})$ we have $\underline{F}^{a, b}(R)<\underline{F}^{a, b}(\bar{R})=p(\bar{R})$ and for the maxima $\bar{F}(R)$ and $\bar{F}(\bar{R})$ we have $\bar{F}(R)=\bar{F}(\bar{R})$. Thus, for each $i \in N \backslash\{j\}$, minimum $\underline{F}^{a, b}(R)<\underline{F}^{a, b}(\bar{R}) \leq$ $p\left(R_{i}\right)$. If maximum $\bar{F}^{a, b}(\bar{R})<p\left(R_{i}\right)$, then $b_{F^{a, b}(R)}\left(R_{i}\right)=b_{F^{a, b}(\bar{R})}\left(R_{i}\right)<p\left(R_{i}\right)$ and $w_{F^{a, b}(R)}\left(R_{i}\right)<w_{F^{a, b}(\bar{R})}\left(R_{i}\right) \leq p\left(R_{i}\right)$. Hence, by single-peakedness, $i$ 's best point is at least as desirable and his worst point improves. If maximum $\bar{F}^{a, b}(\bar{R}) \geq p\left(R_{i}\right)$, then $b_{F^{a, b}(R)}\left(R_{i}\right)=b_{F^{a, b}(\bar{R})}\left(R_{i}\right)=p\left(R_{i}\right)$ and $w_{F^{a, b}(\bar{R})}\left(R_{i}\right) \in F^{a, b}(\bar{R}) \subseteq F^{a, b}(R)$. Thus, $i$ 's best and worst points are at least as desirable. It follows, that for each $i \in N \backslash\{j\}, F^{a, b}(\bar{R}) R_{i} F^{a, b}(R)$.

(iii) $a, b \geq p(\bar{R})$. Then, the set chosen remains the same, $F^{a, b}(\bar{R})=F^{a, b}(R)$.

Case 2.2. $\operatorname{Conv}(p(\bar{R})) \supsetneq \operatorname{Conv}(p(R))$. Then, by Case 2.1 (with the roles of $R$ and $\bar{R}$ reversed), for each $i \in N \backslash\{j\}, F^{a, b}(R) R_{i} F^{a, b}(\bar{R})$.

The next proposition states an important relation between the two solidarity properties we study.

Proposition 2.5 (Efficiency and population-monotonicity $\Rightarrow$ one-sided replacement-dominance). Each choice correspondence satisfying efficiency and population-monotonicity also satisfies one-sided replacement-dominance.

We prove Proposition 2.5 in Appendix 2.C.

Finally, although the property of strategy-proofness ${ }^{22}$ is not within the scope of this chapter, the following remark should be made.

\footnotetext{
${ }^{22}$ An agent cannot affect the chosen set in his favor by misreporting his preferences (a formal definition in a slightly different context can be found on page 79).
} 
Remark 2.4 (Strategy-proofness). Each (fp-)target set correspondence is (essentially) a median correspondence (defined on page 78). Specifically, an (fp)target set correspondence with a target set $\left[a^{N}, b^{N}\right]$, at population $N$, is equivalent with a median correspondence $F^{\alpha, \beta}$, where $\alpha, \beta \in A^{|N|+1}$ such that $\alpha=\left\{-\infty, a^{N}\right.$, $\left.\ldots, a^{N}, \infty\right\}$ and $\beta=\left\{-\infty, b^{N}, \ldots, b^{N}, \infty\right\}$. Therefore, it follows from Theorems 3.4 and 3.6 that each (fp-)target set correspondence satisfies strategyproofness in domains $\mathcal{R}$ and $\mathcal{S}$ respectively.

\subsection{Characterizing target set correspondences}

In the sequel, all results presented refer to single-peaked preferences but also apply to symmetric single-peaked preferences.

Our first theorem states that the properties of efficiency and one-sided replacement-dominance characterize fp-target set correspondences.

Theorem 2.1 ( $F$ is efficient and one-sided replacement-dominant $\Leftrightarrow$ $\left.\boldsymbol{F}=\boldsymbol{F}^{a, b}\right)$. If a fixed population consists of at least 3 agents, then an associated fp-choice correspondence satisfies efficiency and one-sided replacement-dominance if and only if it is an fp-target set correspondence.

We prove Theorem 2.1 in Appendix 2.D. In addition, Corollary 2.3 that follows, strengthens a result for choice functions by Thomson (1993).

Corollary 2.3 ( $F$ is efficient and replacement-dominant $\Leftrightarrow \boldsymbol{F}=\boldsymbol{f}^{a}$ ). If a fixed population consists of at least 3 agents, then an associated fp-choice correspondence satisfies efficiency and replacement-dominance if and only if it is an fp-target point correspondence.

Proof. If part. By Propositions 2.1 and 2.3, all fp-target point correspondences satisfy efficiency and replacement-dominance.

Only if part. Let $N \in \mathcal{P}$ be such that $|N| \geq 3$ and let the fp-choice correspondence $F \in \mathcal{F}^{N}$ satisfy efficiency and replacement-dominance. Then, $F$ satisfies one-sided replacement-dominance and by Theorem 2.1 it is an fp-target set correspondence $F^{a, b} \in \mathcal{F}^{N}$. By Proposition 2.3, $F^{a, b}$ satisfies replacement-dominance if and only if it is an fp-target point correspondence $f^{a} \in \mathcal{F}^{N}$.

We have formulated Theorem 2.1 and Corollary 2.3 for fp-choice correspondences where the fixed population contains at least 3 agents. If instead we consider choice correspondences, then efficiency and one-sided replacement-dominance 
(replacement-dominance) imply that for each population with at least 3 agents, a different target set or target point can be chosen, while for each population with at most 2 agents, the choice correspondence can equal any efficient fp-choice correspondence.

Our second theorem states that the properties of efficiency and populationmonotonicity characterize target set correspondences.

Theorem $2.2\left(\boldsymbol{F}\right.$ is efficient and population-monotonic $\left.\Leftrightarrow \boldsymbol{F}=\boldsymbol{F}^{a, b}\right) . A$ choice correspondence satisfies efficiency and population-monotonicity if and only if it is a target set correspondence.

Proof. If part. By Propositions 2.1 and 2.2, all target set correspondences satisfy efficiency and population-monotonicity.

Only if part. Let choice correspondence $F \in \mathcal{F}$ satisfy efficiency and population-monotonicity. By Proposition 2.5, F satisfies one-sided replacementdominance. Let $M \in \mathcal{P}$ be such that $|M| \geq 3$. By Theorem 2.1, for each $R \in \mathcal{R}^{M}$, $F=F^{a_{M}, b_{M}} \in \mathcal{F}^{M}$. Define points $a:=a_{M}$ and $b:=b_{M}$.

We show that for each $N \in \mathcal{P}$ and each $\bar{R} \in \mathcal{R}^{N}, F(\bar{R})=F^{a, b}(\bar{R})$. We do so by showing that for each $N \in \mathcal{P}$, each $\bar{R} \in \mathcal{R}^{N}$, and each $R \in \mathcal{R}^{M}$, if $\operatorname{Conv}(p(\bar{R}))=\operatorname{Conv}(p(R))$, then $F(\bar{R})=F^{a, b}(R)=F^{a, b}(\bar{R})$ (the latter equality follows by the definition of $\left.F^{a, b}\right)$.

Let $R \in \mathcal{R}^{M}$ and $\bar{R} \in \mathcal{R}^{N}$. Recall that $F(R)=F^{a, b}(R)$. Begin from $R \in \mathcal{R}^{M}$ and construct $R^{1} \in \mathcal{R}^{M \cup N}$ by adding the population $N \backslash M$ with profile $\bar{R}_{N \backslash M}$, i.e., $R^{1}=\left(R, \bar{R}_{N \backslash M}\right)$. Since $\operatorname{Conv}\left(R^{1}\right)=\operatorname{Conv}(p(R))$, by population-monotonicity and Lemma 2.1, $F\left(R^{1}\right)=F(R)$. Next, change the preferences of each $i \in N$ to $\bar{R}_{i}$ and denote the new profile $R^{2}=\left(R_{M \backslash N}^{1}, \bar{R}\right) \in \mathcal{R}^{M \cup N}$. Since $\operatorname{Conv}\left(R^{2}\right)=\operatorname{Conv}\left(R^{1}\right)$, by population-monotonicity and Lemma 2.1, $F\left(R^{2}\right)=F\left(R^{1}\right)$. Finally, remove the population $M \backslash N$ and notice that the new profile $R_{N}^{2}=\bar{R} \in \mathcal{R}^{N}$. Since $\operatorname{Conv}(p(\bar{R}))=\operatorname{Conv}\left(R^{2}\right)$, by population-monotonicity and Lemma 2.1, $F(\bar{R})=$ $F\left(R^{2}\right)$. Hence, $F(\bar{R})=F^{a, b}(R)=F^{a, b}(\bar{R})$.

All the properties we consider are independent.

Remark 2.5 (Independence of properties). Note that the properties in all our characterization results are independent. A constant choice correspondence that always chooses a fixed set satisfies (one-sided) replacement-dominance and population-monotonicity but violates efficiency. A choice correspondence that always chooses the peak of the agent with the lowest index satisfies efficiency, but it violates one-sided replacement-dominance and population-monotonicity. 
Finally, we comment on the validity of our results for some natural model variations.

Remark 2.6 (Chosen sets are not necessarily compact). Although we only study compact subsets of $\mathbb{R}$, the compactness requirement is without loss of generality for the following reasons. First, the agents' peaks being real numbers and Proposition 2.1 (i) imply that unbounded sets are not efficient. Hence, by Theorems 2.1 and 2.2, the two classes of correspondences we characterize satisfy efficiency and therefore only select bounded sets. Second, concerning open (and bounded) sets, after assuming that each agent is indifferent between a set and its closure, ${ }^{23}$ all our results hold and the target sets of target set correspondences and fp-target set correspondences can be open. Notice that in this case, the second requirement for the efficiency of a set, that is, Proposition 2.1 (ii), must change slightly to $\operatorname{Conv}(\operatorname{closure}(X)) \cap p(R) \subseteq \operatorname{closure}(X)$; moreover, to accommodate for the possible openness of sets, throughout the text and for each set $X$, references to $\operatorname{Conv}(X)$ must be substituted with $\operatorname{Conv}(\operatorname{closure}(X))$.

Remark 2.7 (Monotonic preferences). Allowing for agents to have monotonic preferences, i.e., have minus infinity or plus infinity as peaks, poses the following problem. If all agents have minus infinity or all agents have plus infinity as their peak, then by Proposition 2.1, no efficient set exists in $\mathcal{C}$. Moreover, if unbounded sets of $\mathbb{R}$ are considered, then in this case the only efficient sets are $\{-\infty\}$ (when all agents have minus infinity as their peak) and $\{+\infty\}$ (when all agents have plus infinity as their peak). However, a policy interpretation for these two sets, as well as other unbounded sets, is not clear and we therefore do not add monotonic preferences to our model.

Remark 2.8 (Closed interval alternative set). All our results hold if the preferences of the agents are defined on some closed interval $[a, b] \subsetneq \mathbb{R}$. In this case and since efficiency is required, by Proposition 2.1 (i), the class of sets considered equals the class of non-empty subsets of $[a, b]$ and closedness is not required (see Remark 2.6). Moreover, agents can have monotonic preferences, i.e., have $a$ or $b$ as peaks, since the policy interpretation of "locating the public good at $a$ " or "locating the public good at b" is straightforward, in contrast to our original model (see Remark 2.7). Finally, it should be mentioned that this restriction on the set of alternatives facilitates our main proof (Theorem 2.1) as follows. Since a profile with $a$ as the minimum peak and $b$ as the maximum peak can be chosen

\footnotetext{
${ }^{23}$ Given $X \subsetneq \mathbb{R}$, the closure of $X$, closure $(X)$, is defined as the union of $X$ with all its limit / boundary points.
} 
(in contrast to our original model, where a profile with $-\infty$ as the minimum peak and $+\infty$ as the maximum peak is not available), the proof essentially follows from Lemma 2.11.

Throughout the Appendices we use the domain of single-peaked preferences $\mathcal{R}$, with the exception of Lemma 2.9 (Appendix 2.D), where we use the domain of symmetric single-peaked preferences $\mathcal{S}$. All results proven for $\mathcal{R}$ also hold on $\mathcal{S}$; however, for Lemma 2.9, the proof for $\mathcal{S}$ requires a different approach (and additional "proof steps") that also holds on $\mathcal{R}$.

\section{A Proofs of Proposition 2.1 and Corollary 2.2}

The following terms describe a set obtained by a truncation of a given set $X \in \mathcal{C}$ on one side at a specific point $x$, which is added to the new set to ensure that this new set is closed.

Left truncaddition (of a set at a point). Let point $x \in \mathbb{R}$ and set $X \in \mathcal{C}$. Then, set $Y \in \mathcal{C}$ is a left truncaddition of $X$ at $x$ if $Y=[X \cap(x, \infty)] \cup\{x\}$.

Right truncaddition (of a set at a point). Let point $x \in \mathbb{R}$ and set $X \in \mathcal{C}$. Then, set $Y \in \mathcal{C}$ is a right truncaddition of $X$ at $x$ if $Y=[X \cap(-\infty, x)] \cup\{x\}$.

Before proceeding with the proof of Proposition 2.1 we present two lemmas. First, we describe some cases where a truncaddition of a set at a point makes an agent weakly better off.

Lemma 2.3 (Truncadditions). Let agent $i \in \mathbb{P}$ with preferences $R_{i} \in \mathcal{R}$ and set $X \in \mathcal{C}$.

(i) Let minimum $\underline{X}<p\left(R_{i}\right)$, point $\underline{x} \in \mathbb{R}$ such that $\underline{X}<\underline{x} \leq p\left(R_{i}\right)$, and set $Y=[X \cap(\underline{x}, \infty)] \cup\{\underline{x}\}$ be a left truncaddition of set $X$ at point $\underline{x}$. Then, $Y R_{i} X$. Moreover, if the unique worst point $w_{X}\left(R_{i}\right)=X$, then $Y P_{i} X$.

(ii) Let maximum $\bar{X}>p\left(R_{i}\right)$, point $\bar{x} \in \mathbb{R}$ be such that $\bar{X}>\bar{x} \geq p\left(R_{i}\right)$, and set $Y=[X \cap(-\infty, \bar{x})] \cup\{\bar{x}\}$ be a right truncaddition of set $X$ at point $\bar{x}$. Then, $Y R_{i} X$. Moreover, if the unique worst point $w_{X}\left(R_{i}\right)=\bar{X}$, then $Y P_{i} X$.

(iii) Let minimum $X<p\left(R_{i}\right)$, maximum $\bar{X}>p\left(R_{i}\right)$, and points $\underline{x}, \bar{x} \in \mathbb{R}$ be such that $\underline{X}<\underline{x} \leq p\left(R_{i}\right) \leq \bar{x}<\bar{X}$, set $Y=[X \cap(\underline{x}, \infty)] \cup\{\underline{x}\}$ be a left truncaddition of set $X$ at point $\underline{x}$, and set $Z=[Y \cap(-\infty, \bar{x})] \cup\{\bar{x}\}$ be a right truncaddition of set $Y$ at point $\bar{x}$. Then, $Z P_{i} X$.

Proof. Let agent $i \in \mathbb{P}$ with preferences $R_{i} \in \mathcal{R}$ and set $X \in \mathcal{C}$.

(i) Let minimum $\underline{X}<p\left(R_{i}\right)$, point $\underline{x} \in \mathbb{R}$ such that $\underline{X}<\underline{x} \leq p\left(R_{i}\right)$, truncaddition $Y=[X \cap(\underline{x}, \infty)] \cup\{\underline{x}\}$, and $Z$ be the set of truncated points, $Z=X \backslash Y$. 
By single-peakedness, for each $z \in Z$, agent $i$ prefers $\underline{x}$ to $z, \underline{x} P_{i} z$. Hence, his best and worst points in $Y$ are at least as desirable as his (respective) best and worst points in $X$. It follows, that $Y R_{i} X$. If additionally his worst point $w_{X}\left(R_{i}\right)=\underline{X} \notin Y$ is unique, then $\bar{X} P_{i} w_{X}\left(R_{i}\right)$ and $\underline{x} P_{i} w_{X}\left(R_{i}\right)$. Since by singlepeakedness, $w_{Y}\left(R_{i}\right) \subseteq\{\underline{x}, \bar{X}\}$, it follows that $Y P_{i} X$.

(ii) Symmetric proof to (i).

(iii) Let minimum $\underline{X}<p\left(R_{i}\right)$, maximum $\bar{X}>p\left(R_{i}\right)$, points $\underline{x}, \bar{x} \in \mathbb{R}$ be such that $X<\underline{x} \leq p\left(R_{i}\right) \leq \bar{x}<\bar{X}$, truncaddition $Y=[X \cap(\underline{x}, \infty)] \cup\{\underline{x}\}$, and truncaddition $Z=[Y \cap(-\infty, \bar{x})] \cup\{\bar{x}\}$. By part (i), $Y R_{i} X$. By part (ii), $Z R_{i} Y$. Hence, by transitivity, $Z R_{i} X$. Moreover, by single-peakedness, his worst point(s) $w_{X}\left(R_{i}\right) \subseteq\{\underline{X}, \bar{X}\}$ and $w_{Z}\left(R_{i}\right) \subseteq\{\underline{x}, \bar{x}\}$. Since by single-peakedness $\underline{x} P_{i} w_{X}\left(R_{i}\right)$ and $\bar{x} P_{i} w_{X}\left(R_{i}\right)$, his worst point(s) improves. It follows that $Z P_{i} X$.

Second, adding a closed interval to a set, without changing its convex hull, makes an agent indifferent, unless his best point improves, in which case he is better off. Furthermore, removing an open interval from a set, without changing its convex hull, makes an agent indifferent, unless his best point worsens, in which case he is worse off.

Lemma 2.4. Let agent $i \in \mathbb{P}$ with preferences $R_{i} \in \mathcal{R}$ and set $X \in \mathcal{C}$.

(i) Let closed interval $[x, y] \subseteq \operatorname{Conv}(X)$ and set $Y=X \cup[x, y]$. Then, $Y I_{i} X$ unless agent $i$ 's best point(s) improves, i.e., $b_{Y}\left(R_{i}\right) P_{i} b_{X}\left(R_{i}\right)$, in which case, $Y P_{i} X$.

(ii) Let open interval $(x, y) \subsetneq \operatorname{Conv}(X)$ and set $Y=X \backslash(x, y)$. Then, $X I_{i} Y$ unless agent $i$ 's best point(s) worsens, i.e., $b_{X}\left(R_{i}\right) P_{i} b_{Y}\left(R_{i}\right)$, in which case, $X P_{i} Y$.

Proof. Let agent $i \in \mathbb{P}$ with preferences $R_{i} \in \mathcal{R}$ and set $Y \in \mathcal{C}$.

(i) Let $[x, y] \subseteq \operatorname{Conv}(X)$ and $Y=X \cup[x, y]$. By single-peakedness, agent $i$ 's worst point(s) does not change, $w_{X}\left(R_{i}\right)=w_{Y}\left(R_{i}\right) \subseteq\{\underline{X}, \bar{X}\}$. If for his best point(s) we have $b_{X}\left(R_{i}\right) I_{i} b_{Y}\left(R_{i}\right)$, then $b_{X}\left(R_{i}\right) \subseteq b_{Y}\left(R_{i}\right)$ and $Y I_{i} X$. Otherwise, $b_{X}\left(R_{i}\right) \nsubseteq b_{Y}\left(R_{i}\right)$, his best point(s) improves, $b_{Y}\left(R_{i}\right) P_{i} b_{X}\left(R_{i}\right)$, and $Y P_{i} X$.

(ii) Let $(x, y) \subsetneq \operatorname{Conv}(X)$ and $Y=X \backslash(x, y)$. By single-peakedness, agent $i$ 's worst point(s) does not change, $w_{X}\left(R_{i}\right)=w_{Y}\left(R_{i}\right) \subseteq\{\underline{X}, \bar{X}\}$. If for his best point(s) we have $b_{X}\left(R_{i}\right) I_{i} b_{Y}\left(R_{i}\right)$, then $b_{X}\left(R_{i}\right) \supseteq b_{Y}\left(R_{i}\right)$ and $Y I_{i} X$. Otherwise, $b_{X}\left(R_{i}\right) \nsupseteq b_{Y}\left(R_{i}\right)$, his best point(s) worsens, $b_{X}\left(R_{i}\right) P_{i} b_{Y}\left(R_{i}\right)$, and $X P_{i} Y$.

Proof of Proposition 2.1. Let population $N \in \mathcal{P}$, profile $R \in \mathcal{R}^{N}$, and set $X \in \mathcal{C}$. Without loss of generality, assume that $N=\{1, \ldots, n\}$ and $\underline{p}(R)=$ $p\left(R_{1}\right) \leq \ldots \leq p\left(R_{n}\right)=\bar{p}(R)$. The proof follows in three steps. 
Step 1. We show that if set $X \in \mathrm{PE}(R)$ then condition (i) holds, that is, $X \subseteq \operatorname{Conv}(p(R))$.

Let set $X \in \mathrm{PE}(R)$. Assume by contradiction that $X \nsubseteq \operatorname{Conv}(p(R))$. Then, minimum $\underline{X}<p\left(R_{1}\right)$ or maximum $\bar{X}>p\left(R_{n}\right)$. By symmetry of arguments, assume that $X<p\left(R_{1}\right)$.

Case 1. Let maximum $\bar{X}>p\left(R_{n}\right)$. Then, for each $i \in N$, minimum $X<$ $p\left(R_{1}\right) \leq p\left(R_{i}\right) \leq p\left(R_{n}\right)<\bar{X}$. Let $Y=\left[X \cap\left(p\left(R_{1}\right), \infty\right)\right] \cup\left\{p\left(R_{1}\right)\right\}$ be a left truncaddition of $X$ at $p\left(R_{1}\right)$, and $Z=\left[Y \cap\left(-\infty, p\left(R_{n}\right)\right)\right] \cup\left\{p\left(R_{n}\right)\right\}$ be a right truncaddition of $Y$ at $p\left(R_{n}\right)$. Therefore, by Lemma 2.3 (iii), for each $i \in N, Z P_{i} X$. Hence, $X \notin \mathrm{PE}(R)$; a contradiction.

Case 2. Let maximum $\bar{X} \leq p\left(R_{n}\right)$. Then, for each $i \in N$, minimum $X<$ $p\left(R_{1}\right) \leq p\left(R_{i}\right)$. Let $Y=\left[X \cap\left(p\left(R_{1}\right), \infty\right)\right] \cup\left\{p\left(R_{1}\right)\right\}$ be a left truncaddition of $X$ at $p\left(R_{1}\right)$. By Lemma 2.3 (i), for each $i \in N, Y R_{i} X$. Furthermore, agent $n$ 's worst point $w_{X}\left(R_{n}\right)=X$ is unique. Therefore, by Lemma 2.3 (i), $Y P_{n} X$. Hence, $X \notin \mathrm{PE}(R)$; a contradiction.

Step 2. We show that if set $X \in \mathrm{PE}(R)$ then condition (ii) holds, that is, $(\operatorname{Conv}(X) \cap p(R)) \subseteq X$.

Let set $X \in \mathrm{PE}(R)$. By Step 1, $X \subseteq \operatorname{Conv}(p(R))$. Assume by contradiction that $(\operatorname{Conv}(X) \cap p(R)) \nsubseteq X$. Then, there exists agent $j \in N$ such that $p\left(R_{j}\right) \in$ $\operatorname{Conv}(X)$ and $p\left(R_{j}\right) \notin X$.

Let set $Y=X \cup\left\{p\left(R_{j}\right)\right\}$. By Lemma 2.4 (i), for each $i \in N, Y R_{i} X$. Furthermore, agent $j$ 's best point $b_{Y}\left(R_{j}\right)=p\left(R_{j}\right) P_{j} b_{X}\left(R_{j}\right)$. Therefore, by Lemma 2.4 (i), $Y P_{j} X$. Hence, $X \notin \mathrm{PE}(R)$; a contradiction.

Step 3. We show that if conditions (i) and (ii) hold for set $X \in \mathcal{C}$, then $X \in \mathrm{PE}(R)$.

Let set $X \in \mathcal{C}$ be such that $X \subseteq \operatorname{Conv}(p(R))$ and $(\operatorname{Conv}(X) \cap p(R)) \subseteq X$. Assume by contradiction that $X \notin \mathrm{PE}(R)$. Hence, there exists a set $Y \subseteq \mathbb{R}$ that dominates set $X$, i.e., for each agent $i \in N, Y R_{i} X$, and for at least one agent $j \in N, Y P_{j} X$.

Case 1. Let agent $j$ 's peak $p\left(R_{j}\right) \in \operatorname{Conv}(X)$. By condition (ii), $p\left(R_{j}\right) \in X$. Agent $j$ 's best point $b_{X}\left(R_{j}\right)=p\left(R_{j}\right) \in X$ cannot be improved. By singlepeakedness, agent $j$ 's worst point(s) $w_{X}\left(R_{j}\right) \subseteq\{\underline{X}, \bar{X}\}$; if his worst point(s) $w_{Y}\left(R_{j}\right) P_{j} w_{X}\left(R_{j}\right)$, by single-peakedness, minimum $X<\underline{Y}$ or maximum $\bar{X}>\bar{Y}$. By symmetry of arguments, assume minimum $X<\underline{Y}$. Consider agent 1 ; by condition (i), his peak $p\left(R_{1}\right) \leq \underline{X}<\underline{Y}$. By single-peakedness, his best point $b_{X}\left(R_{1}\right) P_{1} b_{Y}\left(R_{1}\right)$. It follows that for agent 1 set $Y$ is not at least as desirable as set $X$. Hence, set $Y$ does not dominate set $X$; a contradiction. 
Case 2. Let agent $j$ 's peak $p\left(R_{j}\right) \notin \operatorname{Conv}(X)$. Then, either $p\left(R_{j}\right)<X$ or $p\left(R_{j}\right)>\bar{X}$. By symmetry of arguments, assume that $p\left(R_{j}\right)>\bar{X}$. By singlepeakedness, agent $j$ 's best point $b_{X}\left(R_{j}\right)=\bar{X}$ and agent $j$ 's worst point $w_{X}\left(R_{j}\right)=$ $X$. If his best point(s) $b_{Y}\left(R_{j}\right) P_{j} b_{X}\left(R_{j}\right)$, by single-peakedness, maximum $\bar{X}<\bar{Y}$. If his worst point(s) $w_{Y}\left(R_{j}\right) P_{j} w_{X}\left(R_{j}\right)$, by single-peakedness, minimum $X<\underline{Y}$. Consider now agent 1. By condition (i), his peak $p\left(R_{1}\right) \leq X \leq \bar{X}$. By singlepeakedness, his best and worst point(s) are $b_{X}\left(R_{1}\right)=X$ and $w_{X}\left(R_{1}\right)=\bar{X}$. If minimum $X<\underline{Y}$, by single-peakedness, $b_{X}\left(R_{1}\right) P_{1} b_{Y}\left(R_{1}\right)$. If maximum $\bar{X}<\bar{Y}$, by single-peakedness, $w_{X}\left(R_{1}\right) P_{1} w_{Y}\left(R_{1}\right)$. It follows that for agent 1 set $Y$ is not at least as desirable as set $X$. Hence, set $Y$ does not dominate set $X$; a contradiction.

Proof of Corollary 2.2. Let population $N \in \mathcal{P}$, profile $R \in \mathcal{R}^{N}$, and set $X \in$ $\mathrm{PE}(R)$.

First, we show that $\operatorname{Conv}(X)$ and $X$ are equivalent sets. By single-peakedness, for each agent $i \in N$ such that $p\left(R_{i}\right) \in \operatorname{Conv}(X)$, the best point $b_{\operatorname{Conv}(X)}\left(R_{i}\right)=$ $p\left(R_{i}\right)$ and by Proposition 2.1 (ii), $(\operatorname{Conv}(X) \cap p(R)) \subseteq X$. Hence, the best point $b_{\operatorname{Conv}(X)}\left(R_{i}\right)=b_{X}\left(R_{i}\right)$. By single-peakedness, for each agent $i \in N$ such that $p\left(R_{i}\right) \notin \operatorname{Conv}(X)$, the best point $b_{\operatorname{Conv}(X)}\left(R_{i}\right) \in\{\underline{X}, \bar{X}\}$. Since $\{\underline{X}, \bar{X}\} \subseteq X$, the best point $b_{\operatorname{Conv}(X)}\left(R_{i}\right)=b_{X}\left(R_{i}\right)$. Moreover, since $\operatorname{Conv}(X)$ is a closed interval and (trivially) $\operatorname{Conv}(X)=X \cup \operatorname{Conv}(X)$, by Lemma 2.4 (i), for each agent $i \in N$, $\operatorname{Conv}(X) I_{i} X$.

Second, we show that if $X$ and $Y$ are equivalent sets, then $\operatorname{Conv}(X)=$ $\operatorname{Conv}(Y)$. Let $Y \in \mathcal{C}$ be an equivalent set to $X \in \operatorname{PE}(R)$. Let agent $1 \in N$ have the smallest peak at profile $R, p\left(R_{1}\right)=\underline{p}(R)$. By Proposition 2.1 (i), $X, Y \subseteq \operatorname{Conv}(p(R))$, hence, $p\left(R_{1}\right) \leq X \leq \bar{X}$ and $p\left(R_{1}\right) \leq \underline{Y} \leq \bar{Y}$. By singlepeakedness, for agent 1 , [best points are $b_{X}\left(R_{1}\right)=\underline{X}$ and $b_{Y}\left(R_{1}\right)=\underline{Y}$ ] and [worst points are $w_{X}\left(R_{1}\right)=\bar{X}$ and $\left.w_{Y}\left(R_{1}\right)=\bar{Y}\right]$. Since $X I_{1} Y, b_{X}\left(R_{1}\right)=b_{Y}\left(R_{1}\right)$ and $w_{X}\left(R_{1}\right)=w_{Y}\left(R_{1}\right)$. Therefore, $\operatorname{Conv}(X)=\operatorname{Conv}(Y)$.

\section{B Proof of Lemma 2.2}

Before proceeding with the proof of Lemma 2.2, we first prove an implication of efficiency and (one-sided) replacement-dominance.

An fp-choice correspondence satisfies extreme-peaks-onliness if the chosen set only depends on the convex hull of the peaks of the profile. We formulate extreme- 
peaks-onliness for fp-choice correspondences but as discussed in Remark 2.3, it easily extends to choice correspondences.

Extreme-peaks-onliness. Let fixed population $N \in \mathcal{P}$ and fp-choice correspondence $F \in \mathcal{F}^{N}$. For each pair of profiles $R, \bar{R} \in \mathcal{R}^{N}$, if $\operatorname{Conv}(p(R))=\operatorname{Conv}(p(\bar{R}))$, then $F(R)=F(\bar{R})$.

Notice that extreme-peaks-onliness not only implies the properties of anonymity ${ }^{24}$ and peaks-onliness ${ }^{25}$ but since it only depends on the extreme agents' peaks, it is a much stronger property.

Lemma 2.5 (Efficiency and one-sided replacement-dominance $\Rightarrow$ extreme-peaks-onliness). If a fixed population consists of at least 3 agents, then each associated fp-choice correspondence satisfying efficiency and one-sided replacement-dominance also satisfies extreme-peaks-onliness.

Proof. Let fixed population $N \in \mathcal{P}$ be such that $|N| \geq 3$ and fp-choice correspondence $F \in \mathcal{F}^{N}$ satisfy efficiency and one-sided replacement-dominance. Let the pair of profiles $R, \bar{R} \in \mathcal{R}^{N}$ be such that $\operatorname{Conv}(p(R))=\operatorname{Conv}(p(\bar{R}))$. Without loss of generality, assume that $N=\{1,2, \ldots, n\}$ and $\underline{p}(R)=p\left(R_{1}\right) \leq p\left(R_{2}\right) \leq$ $\ldots \leq p\left(R_{n}\right)=\bar{p}(R)$. In the following, we refer to agents who have neither the unique smallest peak nor the unique largest peak as middle agents.

We prove that $F(R)=F(\bar{R})$ in three steps.

Step 1. We show that if the preferences of one agent change and the convex hull of the peaks does not change, the chosen set does not change.

Case 1.1. The preferences of a middle agent at profile $R$ change such that the convex hull of the peaks does not change. Let agent $k \in N$ be a middle agent at profile $R$ and let profile $\bar{R} \in \mathcal{R}^{N}$ be such that $\bar{R}_{-k}=R_{-k}$, and $\operatorname{Conv}(p(\bar{R}))=$ $\operatorname{Conv}(p(R))$. Notice that agent $k$ is also a middle agent at profile $\bar{R}^{26}$

By efficiency, $F(\bar{R}) \in P E(\bar{R})$ and $F(R) \in P E(R)$. Since agent $k$ is a middle agent at both profiles $R$ and $\bar{R}, \operatorname{Conv}(p(\bar{R}))=\operatorname{Conv}(p(R))=\operatorname{Conv}\left(p\left(R_{-k}\right)\right)$, and by Corollary 2.1, $F(\bar{R}), F(R) \in \mathrm{PE}\left(R_{-k}\right)$. Since $\bar{R}_{-k}=R_{-k}$, by one-sided replacement-dominance, for each agent $i \in N \backslash\{k\}, F(\bar{R}) R_{i} F(R)$ or for each agent $i \in N \backslash\{k\}, F(R) R_{i} F(\bar{R})$. By efficiency of both sets $F(R)$ and $F(\bar{R})$ at profile $R_{-k}$, for each agent $i \in N \backslash\{k\}, F(R) I_{i} F(\bar{R})$. By Corollary 2.2,

\footnotetext{
${ }^{24}$ Anonymity: the identities of the agents do not affect the chosen set.

${ }^{25}$ Peaks-Onliness: only the peaks of the agents affect the chosen set.

${ }^{26}$ Note that if agent 1 (agent $n$ ) does not have the unique smallest (largest) peak, then he is a middle agent.
} 
$\operatorname{Conv}(F(\bar{R}))=\operatorname{Conv}(F(R))$ and since we always represent any efficient set by its convex hull, $F(\bar{R})=F(R)$.

Case 1.2. Either the preferences of the agent with the unique smallest peak at profiles $R$ and $\bar{R}$ change (agent 1 ), or the preferences of the agent with the unique largest peak at profiles $R$ and $\bar{R}$ change (agent $n$ ), such that the convex hull of the peaks does not change. By symmetry of arguments, assume that profile $\bar{R}$ is such that $\bar{R}_{-1}=R_{-1}$ and $\operatorname{Conv}(p(\bar{R}))=\operatorname{Conv}(p(R))$. Hence, $p\left(\bar{R}_{1}\right)=p\left(R_{1}\right)<$ $p\left(R_{2}\right) \leq \ldots \leq p\left(R_{n}\right)$.

Begin from profile $R$ and construct profile $R^{1}$ by changing middle agent 2 's preferences to $R_{2}^{1}=R_{1}$, i.e., $R^{1}=\left(R_{-2}, R_{2}^{1}\right)$ where $\operatorname{Conv}\left(R^{1}\right)=\operatorname{Conv}(p(R))$. By Case $1.1, F\left(R^{1}\right)=F(R)$. Next, change middle agent 1's preferences to $R_{1}^{2}=\bar{R}_{1}$ such that the new profile is $R^{2}=\left(R_{-1}^{1}, R_{1}^{2}\right)$ where $\operatorname{Conv}\left(R^{2}\right)=\operatorname{Conv}\left(R^{1}\right)$. By Case 1.1, $F\left(R^{2}\right)=F\left(R^{1}\right)$. Finally, change middle agent 2's preferences back to $R_{2}$ and notice that the new profile $\left(R_{-2}^{2}, R_{2}\right)=\bar{R}$ where $\operatorname{Conv}(p(\bar{R}))=\operatorname{Conv}\left(R^{2}\right)$. By Case 1.1, $F(\bar{R})=F\left(R^{2}\right)$. Therefore, $F(\bar{R})=F(R)$.

Step 2. We show that if two agents swap preferences, then the chosen set does not change.

Case 2.1. At least one of the swapping agents is a middle agent at profile $R$. Assume profile $\bar{R}$ is obtained from profile $R$ by agents $j, k \in N$ swapping preferences, i.e., $\bar{R}_{-j, k}=R_{-j, k}, \bar{R}_{j}=R_{k}$, and $\bar{R}_{k}=R_{j}$. Let agent $k \in N$ be a middle agent at profile $R$. Begin from profile $R$ and construct profile $R^{1}$ by changing agent $k$ 's preferences to $R_{k}^{1}=R_{j}$, i.e., $R^{1}=\left(R_{-k}, R_{k}^{1}\right)$ where $\operatorname{Conv}\left(R^{1}\right)=$ $\operatorname{Conv}(p(R))$. By Case 1.1, $F\left(R^{1}\right)=F(R)$. Finally, change agent $j$ 's preferences to $R_{j}^{2}=R_{k}$ and notice that the new profile $\left(R_{-j}^{1}, R_{j}^{2}\right)=\bar{R}$ where $\operatorname{Conv}(p(\bar{R}))=$ $\operatorname{Conv}\left(R^{1}\right)$. By Case 1.1, $F(\bar{R})=F\left(R^{1}\right)$. Therefore, $F(\bar{R})=F(R)$.

Case 2.2. None of the swapping agents is a middle agent at profile $R$. Hence, $p\left(R_{1}\right)<p\left(R_{2}\right) \leq \ldots<p\left(R_{n}\right)$. Note that in this case, $\bar{R} \in \mathcal{R}^{N}$ is such that $\bar{R}_{-1, n}=R_{-1, n}, \bar{R}_{1}=R_{n}$, and $\bar{R}_{n}=R_{1}$. Begin from profile $R$ and construct profile $R^{1}$ by swapping middle agent 2 's preferences with agent 1 's preferences, denoting the new profile by $R^{1}$. By Case 2.1, $F\left(R^{1}\right)=F(R)$. Next, swap middle agent 1 's preferences with agent $n$ 's preferences, denoting the new profile by $R^{2}$. By Case 2.1, $F\left(R^{2}\right)=F\left(R^{1}\right)$. Finally, swap middle agent $n$ 's preferences with agent 2 's preferences and notice that the new profile is $\bar{R}$. By Case 2.1, $F(\bar{R})=F\left(R^{2}\right)$. Therefore, $F(\bar{R})=F(R)$.

Step 3. We show how each profile $\bar{R}$, where $\operatorname{Conv}(p(\bar{R}))=\operatorname{Conv}(p(R))$, can be constructed from profile $R$ by sequentially repeating the first two steps of the proof. Let profile $\bar{R}$ be such that $\bar{R}=\left(\bar{R}_{\overline{1}}, \ldots, \bar{R}_{\bar{n}}\right)$ and, without loss of generality, 
assume $\underline{p}(\bar{R})=p\left(\bar{R}_{\overline{1}}\right) \leq \ldots \leq p\left(\bar{R}_{\bar{n}}\right)=\bar{p}(\bar{R})$. Notice that set $\{\overline{1}, \ldots, \bar{n}\}$ is a permutation of set $N=\{1, \ldots, n\}$.

Begin from profile $R$ and construct profile $R^{1}$ by sequentially replacing each agent' preferences $R_{i}$ with $\bar{R}_{\bar{i}}$, i.e., for each $i \in N, R_{i}^{1}=\bar{R}_{\bar{i}}$. Note that the stepwise change of agents' preferences never changes the convex hull of peaks and that $\operatorname{Conv}\left(R^{1}\right)=\operatorname{Conv}(p(R))$. By Step 1, $F\left(R^{1}\right)=F(R)$. Finally, permute the agents' preferences such that each agent $\bar{i}$ obtains the preferences of agent $i$, i.e., the new profile $R^{2}$ is such that for each $i \in N, R_{\bar{i}}^{2}=R_{i}^{1}$. Hence, for each $i \in N$, $R_{\bar{i}}^{2}=\bar{R}_{\bar{i}}$ and $R^{2}=\bar{R}$. Since all permutations can be obtained via sequential pairwise swaps, by Step 2, $F(\bar{R})=F(R)$.

We use Lemma 2.5 in the proof of Lemma 2.2.

Proof of Lemma 2.2. Let fixed population $N \in \mathcal{P}$ be such that $|N| \geq 3$ and fp-choice correspondence $F \in \mathcal{F}^{N}$ satisfy efficiency and one-sided replacementdominance. By Lemma 2.5, $F$ satisfies extreme-peaks-onliness. Let agent $j \in N$ and the pair of profiles $R, \bar{R} \in \mathcal{R}^{N}$ be such that $R_{-j}=\bar{R}_{-j}$.

We show that if $\operatorname{Conv}(p(\bar{R})) \subseteq \operatorname{Conv}(p(R))$, then all remaining agents end up at least as well off, i.e., for each $i \in N \backslash\{j\}, F(\bar{R}) R_{i} F(R)$. Without loss of generality, assume that $N=\{1,2, \ldots, n\}$ and $\underline{p}(R)=p\left(R_{1}\right) \leq p\left(R_{2}\right) \leq \ldots \leq p\left(R_{n}\right)=\bar{p}(R)$. In the following, we refer to agents who have neither the unique smallest peak nor the unique largest peak as middle agents.

Case 1. Let $\operatorname{Conv}(p(\bar{R}))=\operatorname{Conv}(p(R))$. By extreme-peaks-onliness, $F(\bar{R})=$ $F(R)$.

Case 2. Let $\operatorname{Conv}(p(\bar{R})) \subsetneq \operatorname{Conv}(p(R))$. Hence, at profile $R$, either agent $j=1$ has the unique smallest peak or agent $j=n$ has the unique largest peak. By symmetry of arguments, assume that $j=1$ has the unique smallest peak and profile $\bar{R}$ is such that $\bar{R}_{-1}=R_{-1}$.

Case 2.1. Agent 1 is a middle agent at profile $\bar{R}$. Then, $\operatorname{Conv}(p(\bar{R}))=$ $\operatorname{Conv}\left(p\left(R_{-1}\right)\right)$. By efficiency, $F(\bar{R}) \in P E(\bar{R})$ and $F(R) \in P E(R)$. By Corollary 2.1, $F(\bar{R}) \in P E\left(R_{-1}\right)$.

Assume that $F(R) \subseteq \operatorname{Conv}\left(p\left(R_{-1}\right)\right)$. Since $F(R) \in P E(R)$, by Proposition 2.1 (ii), $\operatorname{Conv}(F(R)) \cap p(R) \subseteq F(R)$. Hence, $\operatorname{Conv}(F(R)) \cap p\left(R_{-1}\right) \subseteq F(R)$ and by Proposition 2.1, $F(R) \in \operatorname{PE}\left(R_{-1}\right)$. Since $\bar{R}_{-1}=R_{-1}$ and $\operatorname{Conv}(p(\bar{R})) \subsetneq$ $\operatorname{Conv}(p(R))$, by one-sided replacement-dominance, for each agent $i \in N \backslash\{1\}$, $F(\bar{R}) R_{i} F(R)$ or for each agent $i \in N \backslash\{1\}, F(R) R_{i} F(\bar{R})$. By efficiency of both sets $F(R)$ and $F(\bar{R})$ at profile $R_{-1}$, for each agent $i \in N \backslash\{1\}, F(R) I_{i} F(\bar{R})$. 
By Corollary 2.2, $\operatorname{Conv}(F(\bar{R}))=\operatorname{Conv}(F(R))$, and since we always represent any efficient set by its convex hull, $F(\bar{R})=F(R)$.

Assume that $F(R) \nsubseteq \operatorname{Conv}\left(p\left(R_{-1}\right)\right)$. Then, minimum $\underline{F}(R)<\underline{p}\left(R_{-1}\right) \leq$ $\underline{F}(\bar{R}) \leq p\left(R_{n}\right)$. Hence, agent $n$ 's worst points are $w_{F(R)}\left(R_{n}\right)=\{\underline{F}(R)\}$ and $w_{F(\bar{R})}\left(R_{n}\right)=\{\underline{F}(\bar{R})\}$. By single-peakedness, $w_{F(\bar{R})}\left(R_{n}\right) P_{n} w_{F(R)}\left(R_{n}\right)$. By onesided replacement-dominance, agent $n$ is better off, $F(\bar{R}) P_{n} F(R)$. Hence, by one-sided replacement-dominance, for each agent $i \in N \backslash\{1\}, F(\bar{R}) R_{i} F(R)$.

Case 2.2. Recall that $\operatorname{Conv}(p(\bar{R})) \subsetneq \operatorname{Conv}(p(R))$ and that agent 1 has the unique smallest peak at profile $R$. In addition, let agent 1 also have the unique smallest peak at profile $\bar{R}$. Then, $\operatorname{Conv}\left(p\left(R_{-1}\right)\right) \subsetneq \operatorname{Conv}(p(\bar{R})) \subsetneq \operatorname{Conv}(p(R))$. Hence, $p\left(R_{1}\right)<p\left(\bar{R}_{1}\right)<p\left(R_{2}\right) \leq \ldots \leq p\left(R_{n}\right)$.

Begin from profile $R$ and construct profile $R^{1}$ by changing middle agent 2's preferences to $R_{2}^{1}=\bar{R}_{1}$, i.e., $R^{1}=\left(R_{-2}, R_{2}^{1}\right)$. Since $\operatorname{Conv}\left(R^{1}\right)=\operatorname{Conv}(p(R))$, by extreme-peaks-onliness, $F\left(R^{1}\right)=F(R)$. Next, change agent 1 's preferences to $R_{1}^{2}=\bar{R}_{1}$ such that the new profile is $R^{2}=\left(R_{-1}^{1}, R_{1}^{2}\right)$. Since agent 1 has the unique smallest peak at profile $R^{1}$ and is a middle agent at profile $R^{2}$, by Case 2.1, for each agent $i \in N \backslash\{1,2\}, F\left(R^{2}\right) R_{i} F\left(R^{1}\right)$. Finally, change middle agent 2 's preferences back to $R_{2}$ and notice that the new profile $\left(R_{-2}^{2}, R_{2}\right)=\bar{R}$. Since $\operatorname{Conv}(p(\bar{R}))=\operatorname{Conv}\left(R^{2}\right)$, by extreme-peaks-onliness, $F(\bar{R})=F\left(R^{2}\right)$. Therefore, for each agent $i \in N \backslash\{1,2\}, F(\bar{R}) R_{i} F(R)$. In particular, $F(\bar{R}) R_{n} F(R)$. Since agent $n$ has the largest peak, efficiency and single-peakedness imply $\underline{F}(R) \leq \underline{F}(\bar{R})$ and $\bar{F}(R) \leq \bar{F}(\bar{R})$. Hence, either $F(\bar{R})=F(R)$ or $F(\bar{R}) P_{n} F(R)$. Then, since $\operatorname{Conv}(p(\bar{R})) \subsetneq \operatorname{Conv}(p(R))$ and $\bar{R}_{-1}=R_{-1}$, by one-sided replacement-dominance, for each agent $i \in N \backslash\{1\}$ (including agent 2 now), $F(\bar{R}) R_{i} F(R)$.

\section{C Proof of Proposition 2.5}

Before proceeding with the proof of Proposition 2.5, we first prove an implication of efficiency and population-monotonicity.

Lemma 2.6. Let choice correspondence $F \in \mathcal{F}$ satisfy efficiency and populationmonotonicity. Then, for each population $N \in \mathcal{P}$ such that $|N| \geq 3$ and each profile $R \in \mathcal{R}^{N}$, the following hold.

(i) Without loss of generality, let agents $1,2 \in N$ where $p\left(R_{1}\right)=\underline{p}(R)$ and $p\left(R_{2}\right)=\underline{p}\left(R_{-1}\right)$. If maximum $\bar{F}(R) \in \operatorname{Conv}\left(p\left(R_{-1}\right)\right)$ and maximum $\bar{F}(R) \in$ $w_{F(R)}\left(R_{2}\right)$, then maxima $\bar{F}(R)=\bar{F}\left(R_{-1}\right)$. Moreover, if $F(R) \subseteq \operatorname{Conv}\left(p\left(R_{-1}\right)\right)$, then $F(R)=F\left(R_{-1}\right)$. 
(ii) Without loss of generality, let agents $n-1, n \in N$ where $p\left(R_{n}\right)=\bar{p}(R)$ and $p\left(R_{n-1}\right)=\bar{p}\left(R_{-n}\right)$. If minimum $\underline{F}(R) \in \operatorname{Conv}\left(p\left(R_{-n}\right)\right)$ and minimum $\underline{F}(R) \in$ $w_{F(R)}\left(R_{n-1}\right)$, then minima $\underline{F}(R)=\underline{F}\left(R_{-n}\right)$. Moreover, if $F(R) \subseteq \operatorname{Conv}\left(p\left(R_{-n}\right)\right)$, then $F(R)=F\left(R_{-n}\right)$.

Proof. Let choice correspondence $F \in \mathcal{F}$ satisfy efficiency and populationmonotonicity. Let population $N \in \mathcal{P}$ be such that $|N| \geq 3$ and profile $R \in \mathcal{R}^{N}$.

(i) Let agents $1,2 \in N$ be such that $p\left(R_{1}\right)=\underline{p}(R)$ and $p\left(R_{2}\right)=\underline{p}\left(R_{-1}\right)$. Let maximum $\bar{F}(R) \in \operatorname{Conv}\left(p\left(R_{-1}\right)\right)$ and maximum $\bar{F}(R) \in w_{F(R)}\left(R_{2}\right)$. Hence, $p\left(R_{2}\right) \leq \bar{F}(R)$. By population-monotonicity and Lemma 2.1, for each agent $i \in$ $N \backslash\{1\}, F\left(R_{-1}\right) R_{i} F(R)$. Let agent $n \in N \backslash\{1,2\}$ have the largest peak at profile $R$, i.e., $p\left(R_{n}\right)=\bar{p}(R)=\bar{p}\left(R_{-1}\right)$. Since agent $n$ has the largest peak at profiles $R$ and $R_{-1}, F\left(R_{-1}\right) R_{n} F(R)$ and efficiency imply $\underline{F}(R) \leq \underline{F}\left(R_{-1}\right) \leq p\left(R_{n}\right)$ and $\bar{F}(R) \leq \bar{F}\left(R_{-1}\right) \leq p\left(R_{n}\right)$. Since agent 2 has the smallest peak at profile $R_{-1}$, $p\left(R_{2}\right) \leq \bar{F}(R)$, and $\bar{F}(R) \in w_{F(R)}\left(R_{2}\right), F\left(R_{-1}\right) R_{1} F(R)$ and efficiency imply $p\left(R_{2}\right) \leq \bar{F}\left(R_{-1}\right) \leq \bar{F}(R)$. Therefore, maxima $\bar{F}(R)=\bar{F}\left(R_{-1}\right)$.

Moreover, let $F(R) \subseteq \operatorname{Conv}\left(p\left(R_{-1}\right)\right)$. Hence, $p\left(R_{2}\right) \leq \underline{F}(R)$. Since agent 2 has the smallest peak at profile $R_{-1}$ and $p\left(R_{2}\right) \leq \underline{F}(R), F\left(R_{-1}\right) R_{1} F(R)$ and efficiency imply $p\left(R_{2}\right) \leq \underline{F}\left(R_{-1}\right) \leq \underline{F}(R)$. Therefore, minima $\underline{F}(R)=\underline{F}\left(R_{-1}\right)$ and thus, $F(R)=F\left(R_{-n}\right)$.

(ii) Symmetric proof to (i).

Proof of Proposition 2.5. Let choice correspondence $F \in \mathcal{F}$ satisfy efficiency and population-monotonicity. Recall that for each population $N \in \mathcal{P}$, each choice correspondence $F \in \mathcal{F}$ specifies an fp-choice correspondence $F \in \mathcal{F}^{N}$. Since for each $N \in \mathcal{P}$ such that $|N| \leq 2$, (one-sided) replacement-dominance imposes no restriction on fp-choice correspondence $F \in \mathcal{F}^{N}$, let $N \in \mathcal{P}$ be such that $|N| \geq 3$.

We show that for each profile $R \in \mathcal{R}^{N}$, if the preferences of an agent $j \in N$ change, such that $R_{-j}=\bar{R}_{-j}$ and $\operatorname{Conv}(p(\bar{R})) \subseteq \operatorname{Conv}(p(R))$, then the other agents whose preferences remained unchanged all end up at least as well off, as they were initially, i.e., for each $i \in N \backslash\{j\}, F(R) R_{i} F(\bar{R}) .{ }^{27}$ Without loss of generality, assume that $N=\{1,2, \ldots, n\}$ and $\underline{p}(R)=p\left(R_{1}\right) \leq p\left(R_{2}\right) \leq \ldots \leq p\left(R_{n}\right)=\bar{p}(R)$. In the following, we refer to agents who have neither the unique smallest peak nor the unique largest peak as middle agents.

Case 1. Let $\operatorname{Conv}(p(\bar{R}))=\operatorname{Conv}(p(R))$.

Case 1.1. Let agent $j$ be a middle agent at both profiles $R$ and $\bar{R}$. Then,

\footnotetext{
${ }^{27}$ Notice that the roles of profiles $R$ and $\bar{R}$ can be reversed, hence the case where $\operatorname{Conv}(p(R)) \subseteq$ $\operatorname{Conv}(p(\bar{R}))$ is also covered.
} 
$\operatorname{Conv}(p(\bar{R}))=\operatorname{Conv}(p(R))=\operatorname{Conv}\left(p\left(R_{-j}\right)\right)$. Remove agent $j$ from profile $R$ to obtain profile $R_{-j}$. Since $\operatorname{Conv}\left(p\left(R_{-j}\right)\right)=\operatorname{Conv}(p(R))$, by population-monotonicity and Lemma 2.1, $F\left(R_{-j}\right)=F(R)$. Next, add agent $j$ with preferences $\bar{R}_{j}$ to obtain profile $\bar{R}$. Since $\operatorname{Conv}(p(\bar{R}))=\operatorname{Conv}\left(p\left(R_{-j}\right)\right)$, by population-monotonicity and Lemma 2.1, $F(\bar{R})=F\left(R_{-j}\right)$. Therefore, $F(\bar{R})=F(R)$.

Case 1.2. Let agent $j$ have the unique smallest (largest) peak at both profiles $R$ and $\bar{R}$. Hence, either agent $j=1$ has the unique smallest peak at both profiles $R$ and $\bar{R}$ or agent $j=n$ has the unique largest peak at both profiles $R$ and $\bar{R}$. By symmetry of arguments, assume that $j=1$ and profile $\bar{R}$ is such that $\bar{R}_{-1}=R_{-1}$. Hence, $p\left(R_{1}\right)=p\left(\bar{R}_{1}\right)<p\left(R_{2}\right) \leq \ldots \leq p\left(R_{n}\right)$.

Begin from profile $R$ and construct profile $R^{1}$ by changing agent 2's preferences to $R_{2}^{1}=R_{1}$, i.e., $R^{1}=\left(R_{-2}, R_{2}^{1}\right)$. Since $\operatorname{Conv}\left(R^{1}\right)=\operatorname{Conv}(p(R))$ and agent 2 is a middle agent at both profiles $R^{1}$ and $R$, by Case 1.1, F( $\left.R^{1}\right)=F(R)$. Next, change agent 1 's preferences to $R_{1}^{2}=\bar{R}_{1}$ such that the new profile is $R^{2}=\left(R_{-1}^{1}, R_{1}^{2}\right)$. Since $\operatorname{Conv}\left(R^{2}\right)=\operatorname{Conv}\left(R^{1}\right)$ and agent 1 is a middle agent at both profiles $R^{2}$ and $R^{1}$, by Case 1.1, $F\left(R^{2}\right)=F\left(R^{1}\right)$. Finally, change agent 2's preferences back to $R_{2}$ and notice that the new profile $\left(R_{-2}^{2}, R_{2}\right)=\bar{R}$. Since $\operatorname{Conv}(p(\bar{R}))=\operatorname{Conv}\left(R^{2}\right)$ and agent 2 is a middle agent at both profiles $\bar{R}$ and $R^{2}$, by Case $1.1, F(\bar{R})=F\left(R^{2}\right)$. Therefore, $F(\bar{R})=F(R)$.

Case 2. Let $\operatorname{Conv}(p(\bar{R})) \subsetneq \operatorname{Conv}(p(R))$. Hence, either agent $j=1$ has the unique smallest peak at profile $R$ or agent $j=n$ has the unique largest peak at profile $R$. By symmetry of arguments, assume that $j=1$ and profile $\bar{R}$ is such that $\bar{R}_{-1}=R_{-1}$.

Case 2.1. Let agent 1 be a middle agent at profile $\bar{R}$. Then, $\operatorname{Conv}(p(\bar{R}))=$ $\operatorname{Conv}\left(p\left(R_{-1}\right)\right)$. Begin from profile $R$ and remove agent 1 from profile $R$ to obtain profile $R_{-1}$. By population-monotonicity and Lemma 2.1, for each agent $i \in$ $N \backslash\{1\}, F\left(R_{-1}\right) R_{i} F(R)$. Next, add agent 1 with preferences $\bar{R}_{1}$ to obtain profile $\bar{R}$. Since $\operatorname{Conv}(p(\bar{R}))=\operatorname{Conv}\left(p\left(R_{-1}\right)\right)$, by population-monotonicity and Lemma 2.1, $F(\bar{R})=F\left(R_{-1}\right)$. Therefore, for each agent $i \in N \backslash\{1\}, F(\bar{R}) R_{i} F(R)$.

Case 2.2. Recall that $\operatorname{Conv}(p(\bar{R})) \subsetneq \operatorname{Conv}(p(R))$ and let agent 1 have the unique smallest peak at profile $R$. In addition, let agent 1 also have the unique smallest peak at profile $\bar{R}$. Then, $\operatorname{Conv}\left(p\left(R_{-1}\right)\right) \subsetneq \operatorname{Conv}(p(\bar{R})) \subsetneq \operatorname{Conv}(p(R))$. Hence, $p\left(R_{1}\right)<p\left(\bar{R}_{1}\right)<p\left(R_{2}\right) \leq \ldots \leq p\left(R_{n}\right)$. The proof of this case proceeds in two parts.

First, we show that for each agent $i \in N \backslash\{1,2\}, F(\bar{R}) R_{i} F(R)$ and $F(\bar{R}) \bar{R}_{1} F(R)$. Begin from profile $R$ and construct profile $R^{1}$ by changing agent 2 's preferences to $R_{2}^{1}=\bar{R}_{1}$, i.e., $R^{1}=\left(R_{-2}, R_{2}^{1}\right)$. Since $\operatorname{Conv}\left(R^{1}\right)=\operatorname{Conv}(p(R))$ and 
agent 2 is a middle agent at both profiles $R^{1}$ and $R$, by Case $1.1, F\left(R^{1}\right)=F(R)$. Next, change agent 1's preferences to $R_{1}^{2}=\bar{R}_{1}$ such that the new profile is $R^{2}=$ $\left(R_{-1}^{1}, R_{1}^{2}\right)$. Since agent 1 is a middle agent at profile $R^{2}$, by Case 2.1 , for each agent $i \in N \backslash\{1\}, F\left(R^{2}\right) R_{i}^{1} F\left(R^{1}\right)$. Hence, for each agent $i \in N \backslash\{1,2\}, F\left(R^{2}\right) R_{i} F\left(R^{1}\right)$ and $F\left(R^{2}\right) \bar{R}_{1} F\left(R^{1}\right)$. Finally, change agent 2's preferences back to $R_{2}$ and notice that the new profile $\left(R_{-2}^{2}, R_{2}\right)=\bar{R}$. Since $\operatorname{Conv}(p(\bar{R}))=\operatorname{Conv}\left(R^{2}\right)$ and agent 2 is a middle agent at both profiles $\bar{R}$ and $R^{2}$, by Case $1.1, F(\bar{R})=F\left(R^{2}\right)$. Therefore, for each agent $i \in N \backslash\{1,2\}, F(\bar{R}) R_{i} F(R)$ and $F(\bar{R}) \bar{R}_{1} F(R)$.

Second, we prove that $F(\bar{R}) R_{2} F(R)$. Since agent $n$ has the largest peak at both profiles $R$ and $\bar{R}, F(\bar{R}) R_{n} F(R)$ and efficiency imply $\underline{F}(R) \leq \underline{F}(\bar{R}) \leq p\left(R_{n}\right)$ and $\bar{F}(R) \leq \bar{F}(\bar{R}) \leq p\left(R_{n}\right)$. Hence, either $F(\bar{R})=F(R)$ or $F(\bar{R}) P_{n} F(R)$. If $F(\bar{R})=F(R)$, then $F(\bar{R}) R_{2} F(R)$. If $F(\bar{R}) P_{n} F(R)$, then (a) $\underline{F}(R)<\underline{F}(\bar{R}) \leq$ $p\left(R_{n}\right)$ or $(\mathrm{b}) \bar{F}(R)<\bar{F}(\bar{R}) \leq p\left(R_{n}\right)$.

If $\underline{F}(R) \geq p\left(R_{2}\right)$, then $F(\bar{R}) \subseteq \operatorname{Conv}\left(p\left(R_{-1}\right)\right)$ and by Lemma 2.6 (i), $F(\bar{R})=F\left(R_{-1}\right)$. Next, consider the change from profile $R$ to $R_{-1}$. By populationmonotonicity and Lemma 2.1, for each agent $i \in N \backslash\{1\}, F\left(R_{-1}\right) R_{i} F(R)$. Therefore, for each agent $i \in N \backslash\{1\}$ (including agent 2 now), $F(\bar{R}) R_{i} F(R)$.

The remaining case is that $\underline{F}(R)<p\left(R_{2}\right)$. Since agent 1 has the smallest peak at profile $\bar{R}$, efficiency implies $p\left(\bar{R}_{1}\right) \leq \underline{F}(\bar{R}) \leq \bar{F}(\bar{R})$. If (a) $\underline{F}(R)<\underline{F}(\bar{R})$, then $F(\bar{R}) \bar{R}_{1} F(R)$ implies $\underline{F}(R)<p\left(\bar{R}_{1}\right)$ and if (b) $\bar{F}(R)<\bar{F}(\bar{R})$, then $F(\bar{R}) \bar{R}_{1} F(R)$ implies $\bar{F}(R)<p\left(\bar{R}_{1}\right)$ and thus, $\underline{F}(R)<p\left(\bar{R}_{1}\right)$.

Hence, there are two cases $(2.2 . \alpha) \underline{F}(R)<p\left(\bar{R}_{1}\right) \leq \underline{F}(\bar{R})<p\left(R_{2}\right)$ and $\bar{F}(R)=$ $\bar{F}(\bar{R})$ and $(2.2 . \beta) \underline{F}(R) \leq \bar{F}(R)<p\left(\bar{R}_{1}\right) \leq \underline{F}(\bar{R})<p\left(R_{2}\right)$.

Case 2.2. $\alpha$. If $\bar{F}(R)=\bar{F}(\bar{R}) \leq p\left(R_{2}\right)$, then $b_{F(R)}\left(R_{2}\right)=\bar{F}(R)=\bar{F}(\bar{R})=$ $b_{F(\bar{R})}\left(R_{2}\right) \leq p\left(R_{2}\right)$ and $w_{F(R)}\left(R_{2}\right)=\underline{F}(R)<\underline{F}(\bar{R})=w_{F(\bar{R})}\left(R_{2}\right)<p\left(R_{2}\right)$. By single-peakedness, $F(\bar{R}) P_{2} F(R)$.

If $\bar{F}(R)=\bar{F}(\bar{R})>p\left(R_{2}\right)$, then $b_{F(R)}\left(R_{2}\right)=b_{F(\bar{R})}\left(R_{2}\right)=p\left(R_{2}\right), w_{F(R)}\left(R_{2}\right) \in$ $\{\underline{F}(R), \bar{F}(R)\}$, and $w_{F(\bar{R})}\left(R_{2}\right) \in\{\underline{F}(\bar{R}), \bar{F}(\bar{R})\}$. Then, $\underline{F}(R)<\underline{F}(\bar{R})<p\left(R_{2}\right)<$ $\bar{F}(R)=\bar{F}(\bar{R})$ and single-peakedness imply $F(\bar{R}) R_{2} F(R)$.

Case 2.2. $\beta$. Notice that $b_{F(R)}\left(R_{2}\right)=\{\bar{F}(R)\}$ and $w_{F(R)}\left(R_{2}\right)=\{\underline{F}(R)\}$.

If $\bar{F}(\bar{R}) \leq p\left(R_{2}\right)$, then $\bar{F}(\bar{R}) \in b_{F(\bar{R})}\left(R_{2}\right)$ and $\underline{F}(\bar{R}) \in w_{F(\bar{R})}\left(R_{2}\right)$. Since then $\underline{F}(R) \leq \bar{F}(R)<\underline{F}(\bar{R}) \leq \bar{F}(\bar{R}) \leq p\left(R_{2}\right)$, by single-peakedness, $F(\bar{R}) P_{2} F(R)$.

If $\bar{F}(\bar{R})>p\left(R_{2}\right)$, then $b_{F(\bar{R})}\left(R_{2}\right)=\left\{p\left(R_{2}\right)\right\}$ and $w_{F(\bar{R})}\left(R_{2}\right) \subseteq\{\underline{F}(\bar{R}), \bar{F}(\bar{R})\}$. Hence, $b_{F(\bar{R})}\left(R_{2}\right) P_{2} b_{F(R)}\left(R_{2}\right)$. Since $\underline{F}(R)<\underline{F}(\bar{R})<p\left(R_{2}\right)$, by single-peakedness, $\underline{F}(\bar{R}) P_{2} \underline{F}(R)=w_{F(R)}\left(R_{2}\right)$.

If $\underline{F}(\bar{R}) \in w_{F(\bar{R})}\left(R_{2}\right)$, then $w_{F(\bar{R})}\left(R_{2}\right) P_{2} w_{F(R)}\left(R_{2}\right)$ and $F(\bar{R}) P_{2} F(R)$.

Finally, if $\underline{F}(\bar{R}) \notin w_{F(\bar{R})}\left(R_{2}\right)$, then $w_{F(\bar{R})}\left(R_{2}\right)=\{\bar{F}(\bar{R})\}$. Note that $\bar{F}(\bar{R}) \in$ 
$\operatorname{Conv}\left(p\left(R_{-1}\right)\right)$. By Lemma $2.6(\mathrm{i}), \bar{F}(\bar{R})=\bar{F}\left(R_{-1}\right)$. Consider the change from profile $R$ to $R_{-1}$. By population-monotonicity and Lemma 2.1, for each agent $i \in N \backslash\{1\}, F\left(R_{-1}\right) R_{i} F(R)$. In particular, $F\left(R_{-1}\right) R_{2} F(R)$ and $w_{F\left(R_{-1}\right)}\left(R_{2}\right) R_{2}$ $w_{F(R)}\left(R_{2}\right)$. Since agent 2 has the smallest peak at profile $R_{-1}$, efficiency and single-peakedness imply that $\bar{F}\left(R_{-1}\right) \in w_{F\left(R_{-1}\right)}\left(R_{2}\right)$. Hence, $\bar{F}(\bar{R}) \in w_{F\left(R_{-1}\right)}\left(R_{2}\right)$ and $w_{F(\bar{R})}\left(R_{2}\right)=\bar{F}(\bar{R}) R_{2} w_{F(R)}\left(R_{2}\right)$. Therefore, $F(\bar{R}) R_{2} F(R)$.

\section{D Proof of Theorem 2.1}

Before proceeding with the proof of Theorem 2.1, we first prove some implications of efficiency and (one-sided) replacement-dominance. The first implication is peakmonotonicity, introduced by Ching (1994). The definition follows.

An fp-choice correspondence satisfies peak-monotonicity if whenever an agent's preferences change such that his peak moves to the left (right), the chosen set moves to the left (right). We formulate peak-monotonicity for fp-choice correspondences but as discussed in Remark 2.3, it easily extends to choice correspondences.

Peak-monotonicity. Let fixed population $N \in \mathcal{P}$ and fp-choice correspondence $F \in \mathcal{F}^{N}$. For each agent $j \in N$ and each pair of profiles $R, \bar{R} \in \mathcal{R}^{N}$ such that $R_{-j}=\bar{R}_{-j}$,

$$
\text { if } p\left(\bar{R}_{j}\right) \leq p\left(R_{j}\right) \text {, then }\left\{\begin{array}{c}
\text { minimum } \underline{F}(\bar{R}) \leq \underline{F}(R) \\
\text { and } \\
\text { maximum } \bar{F}(\bar{R}) \leq \bar{F}(R) \text {. }
\end{array}\right.
$$

Lemma 2.7 (Efficiency and one-sided replacement-dominance $\Rightarrow$ peakmonotonicity). If a fixed population consists of at least 3 agents, then an associated fp-choice correspondence that satisfies efficiency and one-sided replacementdominance also satisfies peak-monotonicity.

Proof. Let fixed population $N \in \mathcal{P}$ such that $|N| \geq 3$ and fp-choice correspondence $F \in \mathcal{F}^{N}$ satisfy efficiency and one-sided replacement-dominance. Let agent $j \in N$ and the pair of profiles $R, \bar{R} \in \mathcal{R}^{N}$ be such that $R_{-j}=\bar{R}_{-j}$ and $p\left(\bar{R}_{j}\right) \leq p\left(R_{j}\right)$. By efficiency, $F(R) \in \mathrm{PE}(R)$ and $F(\bar{R}) \in \mathrm{PE}(\bar{R})$. In the following, we refer to agents who have neither the unique smallest peak nor the unique largest peak as middle agents.

Case 1. Let agent $j$ be a middle agent or have the smallest peak at profile $R$. Hence, $\underline{p}(\bar{R}) \leq \underline{p}(R) \leq \bar{p}(\bar{R})=\bar{p}(R)$ and $\operatorname{Conv}(p(R)) \subseteq \operatorname{Conv}(p(\bar{R}))$. By 
one-sided replacement-dominance and Lemma 2.2, for each agent $i \in N \backslash\{j\}$, $F(R) R_{i} F(\bar{R})$. Finally, let agent $n \in N \backslash\{j\}$ have the largest peak at profile $R$, i.e., $p\left(R_{n}\right)=\bar{p}(R)=\bar{p}(\bar{R})$. By $F(R) R_{n} F(\bar{R})$ and efficiency, $\underline{F}(\bar{R}) \leq \underline{F}(R) \leq p\left(R_{n}\right)$ and $\bar{F}(\bar{R}) \leq \bar{F}(R) \leq p\left(R_{n}\right)$.

Case 2. Let agent $j$ have the unique largest peak at profile $R$.

Case 2.1. Let agent $j$ have the unique largest peak at profile $R$ and be a middle agent at profile $\bar{R}$. Hence, $\underline{p}(\bar{R})=\underline{p}(R) \leq \bar{p}(\bar{R})<\bar{p}(R)$. By the symmetric argument of Case 1 (with agent $n$ being a middle agent at profile $\bar{R}$ instead of agent 1 being a middle agent at profile $R$, and with agent $n$ 's peak moving to the right instead of agent 1's peak moving to the left), $\underline{F}(\bar{R}) \leq \underline{F}(R)$ and $\bar{F}(\bar{R}) \leq \bar{F}(R)$.

Case 2.2. Let agent $j$ have the unique largest peak at profile $R$ and the unique smallest peak at profile $\bar{R}$. Hence, $\underline{p}(\bar{R})<\underline{p}(R) \leq \bar{p}(\bar{R})<\bar{p}(R)$. Begin from profile $R$ and construct profile $R^{1}$ by changing agent $j$ 's preferences to $R_{j}^{1}$ such that his peak $p\left(R_{j}^{1}\right)=\underline{p}(R)$, i.e., $R^{1}=\left(R_{-j}, R_{j}^{1}\right)$. Since agent $j$ has the unique largest peak at profile $R$ and is a middle agent at profile $R^{1}$, by Case $2.1, \underline{F}\left(R^{1}\right) \leq \underline{F}(R)$ and $\bar{F}\left(R^{1}\right) \leq \bar{F}(R)$. Finally, change agent $j$ 's preferences to $\bar{R}_{j}$ and notice that the new profile $\left(R_{-j}^{1}, \bar{R}_{j}\right)=\bar{R}$. Since agent $j$ is a middle agent at profile $R^{1}$, by Case $1, \underline{F}(\bar{R}) \leq \underline{F}\left(R^{1}\right) \leq \underline{F}(R)$ and $\bar{F}(\bar{R}) \leq \bar{F}\left(R^{1}\right) \leq \bar{F}(R)$.

The second implication of efficiency and (one-sided) replacement-dominance is uncompromisingness, introduced by Border and Jordan (1983). The definition follows.

Loosely speaking, an fp-choice correspondence satisfies uncompromisingness if whenever an agent's preferences change such that his peaks, before and after this change, both lie on the same side of the minimum (maximum) point chosen, the minimum (maximum) point chosen does not change. We formulate uncompromisingness - and later set-uncompromisingness - for fp-choice correspondences but as discussed in Remark 2.3, they easily extend to choice correspondences.

Uncompromisingness. Let fixed population $N \in \mathcal{P}$ and fp-choice correspondence $F \in \mathcal{F}^{N}$. For each agent $j \in N$ and each pair of profiles $R, \bar{R} \in \mathcal{R}^{N}$ such that $R_{-j}=\bar{R}_{-j}$,

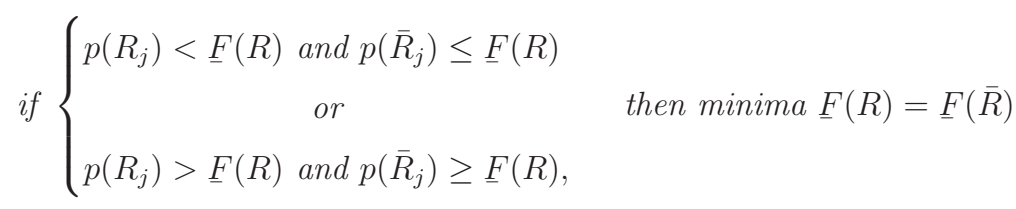

and 


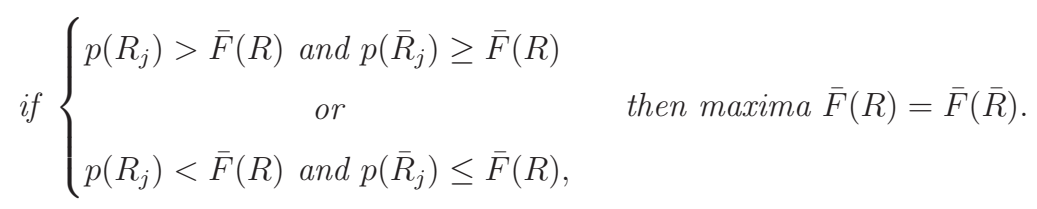

Uncompromisingness immediately implies the following notion of setuncompromisingness.

Set-uncompromisingness. Let fixed population $N \in \mathcal{P}$ and fp-choice correspondence $F \in \mathcal{F}^{N}$. For each agent $j \in N$ and each pair of profiles $R, \bar{R} \in \mathcal{R}^{N}$ such that $R_{-j}=\bar{R}_{-j}$,

$$
\text { if }\left\{\begin{array}{l}
p\left(R_{j}\right)<\underline{F}(R) \text { and } p\left(\bar{R}_{j}\right) \leq \underline{F}(R) \\
\text { or } \\
p\left(R_{j}\right)>\bar{F}(R) \text { and } p\left(\bar{R}_{j}\right) \geq \bar{F}(R),
\end{array} \text { then } F(R)=F(\bar{R}) .\right.
$$

Lemma 2.8 (Uncompromisingness $\Rightarrow$ set-uncompromisingness). Each fp-choice correspondence satisfying uncompromisingness also satisfies setuncompromisingness.

Proof. Follows trivially by the definitions of uncompomisingness and setuncompromisingness.

Before stating in Lemma 2.10 some conditions under which an fp-choice correspondence satisfies uncompromisingness, we first state a result for the domain of symmetric single-peaked preferences $\mathcal{S}$ (Lemma 2.9). This is the only result where we have to change the proof technique when dealing with domain $\mathcal{S}{ }^{28}$ Specifically, we prove Lemma 2.9 using a so-called "leapfrogging" argument. During each leapfrog we right (left) extend the convex hull of the peaks by some distance and if this distance is not enough we repeat this argument as many (finite) times as necessary. Notice that Lemma 2.9 also holds on the domain of single-peaked preferences $\mathcal{R}$.

Lemma 2.9. Let fixed population $N \in \mathcal{P}$ be such that $|N| \geq 3$ and fp-choice correspondence $F \in \mathcal{F}^{N}$ satisfy efficiency and one-sided replacement-dominance. For each agent $j \in N$ and each pair of profiles $R, \bar{R} \in \mathcal{R}^{N}$ such that $R_{-j}=\bar{R}_{-j}$ and $\operatorname{Conv}(p(R)) \subsetneq \operatorname{Conv}(p(\bar{R}))$,

\footnotetext{
${ }^{28}$ Recall that all steps in all other proofs are for domain $\mathcal{R}$ but they automatically apply to domain $\mathcal{S}$.
} 
(i) if minimum $\underline{F}(R)<\bar{p}(R)<p\left(\bar{R}_{j}\right)$, then minima $\underline{F}(\bar{R})=\underline{F}(R)$. Moreover, if also maximum $\bar{F}(R)<\bar{p}(R)$, then $F(\bar{R})=F(R)$,

(ii) if maximum $\bar{F}(R)>\underline{p}(R)>p\left(\bar{R}_{j}\right)$, then maxima $\bar{F}(\bar{R})=\bar{F}(R)$. Moreover, if also minimum $\underline{F}(R)>p(R)$, then $F(\bar{R})=F(R)$.

Proof. Let fixed population $N \in \mathcal{P}$ be such that $|N| \geq 3$ and fp-choice correspondence $F \in \mathcal{F}^{N}$ satisfy efficiency and one-sided replacement-dominance. By Lemmas 2.5 (Appendix 2.B) and 2.7, F satisfies extreme-peaks-onliness and peakmonotonicity.

Let agent $j \in N$ and the pair of profiles $R, \bar{R} \in \mathcal{R}^{N}$ be such that $R_{-j}=\bar{R}_{-j}$ and $\operatorname{Conv}(p(R)) \subsetneq \operatorname{Conv}(p(\bar{R}))$. By efficiency, $F(R) \in \mathrm{PE}(R)$. By extreme-peaksonliness, it is without loss of generality to assume that both profiles $R$ and $\bar{R}$ are symmetric, i.e., $R, \bar{R} \in \mathcal{S}^{N} .{ }^{29}$ In the following, we refer to agents who have neither the unique smallest peak nor the unique largest peak as middle agents. Moreover, we only prove (i) since the proof of (ii) is symmetric.

Let minimum $\underline{F}(R)<\bar{p}(R)<p\left(\bar{R}_{j}\right)$. Since $\bar{p}(R)<p\left(\bar{R}_{j}\right)$ and $F(R) \in \mathrm{PE}(R)$, by Proposition 2.1 (i), $\underline{p}(R) \leq \underline{F}(R) \leq \bar{F}(R) \leq \bar{p}(R)<p\left(\bar{R}_{j}\right)$. Since also $\operatorname{Conv}(p(R)) \subsetneq \operatorname{Conv}(p(\bar{R}))$, agent $j$ either [is a middle agent at profile $R$ and has the unique largest peak at profile $\bar{R}$ ] or [has the unique largest peak at both profiles $R$ and $\bar{R}$ ].

Case 1. Let agent $j$ be a middle agent at profile $R$ and have the unique largest peak at profile $\bar{R}$. Let agent $n \in N \backslash\{j\}$ have the largest peak at profile $R$, i.e., $p\left(R_{n}\right)=\bar{p}(R)$. Hence, minimum $\underline{F}(R)<\bar{p}(R)$ and efficiency imply $\underline{F}(R)<p\left(R_{n}\right)$ and $\bar{F}(R) \leq p\left(R_{n}\right)$. By single-peakedness, $b_{F(R)}\left(R_{n}\right)=\bar{F}(R)$ and $w_{F(R)}\left(R_{n}\right)=$ $\underline{F}(R)$.

Let the distance between minimum $\underline{F}(R)$ and peak $p\left(R_{n}\right)$ be $\delta_{0}=\mid \underline{F}(R)-$ $p\left(R_{n}\right) \mid$. Let point $x_{1} \in \mathbb{R}$ be on the right side of peak $p\left(R_{n}\right)$, i.e., $x_{1}>p\left(R_{n}\right)=$ $\bar{p}(R)$, such that the distance between minimum $\underline{F}(R)$ and point $x_{1}$ is $\delta_{1}=\mid \underline{F}(R)-$ $x_{1} \mid=\frac{3}{2} \delta_{0}$. Hence, distance $\left|p\left(R_{n}\right)-x_{1}\right|=\left|\underline{F}(R)-x_{1}\right|-\left|\underline{F}(R)-p\left(R_{n}\right)\right|=\frac{1}{2} \delta_{0}=$ $\frac{1}{2}\left|\underline{F}(R)-p\left(R_{n}\right)\right|$ and point $x_{1}$ is closer to peak $p\left(R_{n}\right)$ than minimum $\underline{F}(R)$ is.

Step 1. Begin from profile $R$ and construct profile $R^{1}$ by changing agent $j$ 's preferences to $R_{j}^{1} \in \mathcal{S}$ such that his peak

$$
p\left(R_{j}^{1}\right)= \begin{cases}p\left(\bar{R}_{j}\right) & \text { if } p\left(\bar{R}_{j}\right) \leq x_{1} \\ x_{1} & \text { otherwise }\end{cases}
$$

\footnotetext{
${ }^{29}$ For each agent $i \in N$, we can replace preferences $R_{i}, \bar{R}_{i} \in \mathcal{R}$ by preferences $R_{i}^{\prime}, \bar{R}_{i}^{\prime} \in \mathcal{S}$ such that $p\left(R_{i}\right)=p\left(R_{i}^{\prime}\right)$ and $p\left(\bar{R}_{i}\right)=p\left(\bar{R}_{i}^{\prime}\right)$. Then, by extreme-peaks-onliness, $F(R)=F\left(R^{\prime}\right)$ and $F(\bar{R})=F\left(\bar{R}^{\prime}\right)$.
} 
i.e., $R^{1}=\left(R_{-j}, R_{j}^{1}\right)$. Hence, $R_{-j}^{1}=R_{-j}$. By efficiency and Proposition 2.1 (i), $\underline{p}(R)=\underline{p}\left(R^{1}\right) \leq \underline{F}\left(R^{1}\right) \leq \bar{F}\left(R^{1}\right) \leq \bar{p}\left(R^{1}\right)=p\left(\bar{R}_{j}\right)$. Since $p\left(R_{j}^{1}\right)>p\left(R_{j}\right)$, by peak-monotonicity, minimum $\underline{F}\left(R^{1}\right) \geq \underline{F}(R)$ and maximum $\bar{F}\left(R^{1}\right) \geq \bar{F}(R)$. Hence, $\underline{F}\left(R^{1}\right) \in\left[\underline{F}(R), p\left(\bar{R}_{j}\right)\right]$ and $\bar{F}\left(R^{1}\right) \in\left[\bar{F}(R), p\left(\bar{R}_{j}\right)\right]$. Since $\operatorname{Conv}(p(R)) \subsetneq$ $\operatorname{Conv}\left(R^{1}\right)$, by one-sided replacement-dominance and Lemma 2.2, agent $n$ ends up at most as well off, $F(R) R_{n} F\left(R^{1}\right)$. Hence, $b_{F(R)}\left(R_{n}\right) R_{n} b_{F\left(R^{1}\right)}\left(R_{n}\right)$ and $w_{F(R)}\left(R_{n}\right) R_{n} w_{F\left(R^{1}\right)}\left(R_{n}\right)$.

If $\underline{F}\left(R^{1}\right) \in\left[p\left(R_{n}\right), p\left(\bar{R}_{j}\right)\right]$, then $w_{F\left(R^{1}\right)}\left(R_{n}\right)=\bar{F}\left(R^{1}\right) \in\left[p\left(R_{n}\right), p\left(\bar{R}_{j}\right)\right]$. The distance of agent $n$ 's worst point $\bar{F}\left(R^{1}\right)$ to peak $p\left(R_{n}\right)$ is $\left|p\left(R_{n}\right)-\bar{F}\left(R^{1}\right)\right| \leq$ $\left|p\left(R_{n}\right)-p\left(R_{j}^{1}\right)\right| \leq\left|p\left(R_{n}\right)-x_{1}\right|=\frac{1}{2} \delta_{0}=\frac{1}{2}\left|\underline{F}(R)-p\left(R_{n}\right)\right|$, which is smaller than the distance of minimum $\underline{F}(R)$ to peak $p\left(R_{n}\right)$. By symmetric single-peakedness, agent $n$ prefers $w_{F\left(R^{1}\right)}\left(R_{n}\right)=\bar{F}\left(R^{1}\right)$ to $w_{F(R)}\left(R_{n}\right)=\underline{F}(R)$; a contradiction. Hence, $\underline{F}\left(R^{1}\right) \in\left[\underline{F}(R), p\left(R_{n}\right)\right)$ and $w_{F\left(R^{1}\right)}\left(R_{n}\right)=\underline{F}\left(R^{1}\right)$. Since $\underline{F}\left(R^{1}\right)<p\left(R_{n}\right)$, for agent $n$ to find $w_{F(R)}\left(R_{n}\right)=\underline{F}(R)$ at least as desirable as $w_{F\left(R^{1}\right)}\left(R_{n}\right)=\underline{F}\left(R^{1}\right)$, then minimum $\underline{F}(R) \geq \underline{F}\left(R^{1}\right)$. Hence, minima $\underline{F}\left(R^{1}\right)=\underline{F}(R)$.

Moreover, let maximum $\bar{F}(R)<\bar{p}(R)=p\left(R_{n}\right)$. Then, $b_{F(R)}\left(R_{n}\right)=\bar{F}(R)$. Recall that $\bar{F}\left(R^{1}\right) \in\left[\bar{F}(R), p\left(\bar{R}_{j}\right)\right]$. If $\bar{F}\left(R^{1}\right) \in\left[p\left(R_{n}\right), p\left(\bar{R}_{j}\right)\right]$, then agent $n$ prefers $b_{F\left(R^{1}\right)}\left(R_{n}\right)=p\left(R_{n}\right)$ to $b_{F(R)}\left(R_{n}\right)=\bar{F}(R)$; a contradiction. Hence, $\bar{F}\left(R^{1}\right) \in$ $\left[\bar{F}(R), p\left(R_{n}\right)\right)$ and $b_{F\left(R^{1}\right)}\left(R_{n}\right)=\bar{F}\left(R^{1}\right)$. Since $\bar{F}\left(R^{1}\right)<p\left(R_{n}\right)$, for agent $n$ to find $b_{F(R)}\left(R_{n}\right)=\bar{F}(R)$ at least as desirable as $b_{F\left(R^{1}\right)}\left(R_{n}\right)=\bar{F}\left(R^{1}\right)$, then maximum $\bar{F}(R) \geq \bar{F}\left(R^{1}\right)$. Hence, maxima $\bar{F}\left(R^{1}\right)=\bar{F}(R)$ and $F\left(R^{1}\right)=F(R)$.

If $p\left(R_{j}^{1}\right)=p\left(\bar{R}_{j}\right)$, then $\operatorname{Conv}\left(R^{1}\right)=\operatorname{Conv}(p(\bar{R}))$ and by extreme-peaksonliness, $F\left(R^{1}\right)=F(\bar{R})$ and we are done. If $p\left(R_{j}^{1}\right) \neq p\left(\bar{R}_{j}\right)$, then note that agent $n$ is now a middle agent and agent $j$ has the unique largest peak at profile $R^{1}$. We now explain the term "leapfrogging" in order to explain the proof technique: in Step 1, the peak of agent $j$ moves to the right of agent $n$ 's peak by figuratively leapfrogging over agent $n$. In Step 2, the roles of agents $j$ and $n$ reverse, and agent $n$ leapfrogs over agent $j$ to the right, etc.

Let point $x_{2} \in \mathbb{R}$ be on the right side of peak $p\left(R_{j}^{1}\right)$, i.e., $x_{2}>p\left(R_{j}^{1}\right)=\bar{p}\left(R^{1}\right)$, such that the distance between minimum $\underline{F}(R)$ and point $x_{2}$ is $\delta_{2}=\left|\underline{F}(R)-x_{2}\right|=$ $\frac{3}{2} \delta_{1}$. Hence, distance $\left|p\left(R_{j}^{1}\right)-x_{2}\right|=\left|\underline{F}(R)-x_{2}\right|-\left|\underline{F}(R)-p\left(R_{j}^{1}\right)\right|=\frac{1}{2} \delta_{1}=$ $\frac{1}{2}\left|\underline{F}(R)-p\left(R_{j}^{1}\right)\right|$ and point $x_{2}$ is closer to peak $p\left(R_{j}^{1}\right)$ than minimum $\underline{F}(R)$ is.

Step 2. Begin from profile $R^{1}$ and construct profile $R^{2}$ by changing agent $n$ 's preferences to $R_{n}^{2} \in \mathcal{S}$ such that his peak

$$
p\left(R_{n}^{2}\right)= \begin{cases}p\left(\bar{R}_{j}\right) & \text { if } p\left(\bar{R}_{j}\right) \leq x_{2} \\ x_{2} & \text { otherwise }\end{cases}
$$


i.e., $R^{2}=\left(R_{-n}^{1}, R_{n}^{2}\right)$. Hence, $R_{-n}^{2}=R_{-n}^{1}$. By the arguments described in the previous step (with profiles $R$ and $R^{1}$ replaced by profiles $R^{1}$ and $R^{2}$ and with agent $n$ in the role of agent $j$ ), minima $\underline{F}\left(R^{2}\right)=\underline{F}\left(R^{1}\right)=\underline{F}(R)$.

Moreover, let maximum $\bar{F}(R)<\bar{p}(R)$. Then, maximum $\bar{F}(R)=\bar{F}\left(R^{1}\right)<$ $\bar{p}\left(R^{1}\right)=p\left(R_{j}^{1}\right)$ and by the arguments described in the previous step (with profiles $R$ and $R^{1}$ replaced by profiles $R^{1}$ and $R^{2}$ and with agent $n$ in the role of agent $j$ ), $F\left(R^{2}\right)=F\left(R^{1}\right)=F(R)$.

If $p\left(R_{n}^{2}\right)=p\left(\bar{R}_{j}\right)$, then $\operatorname{Conv}\left(R^{2}\right)=\operatorname{Conv}(p(\bar{R}))$ and by extreme-peaksonliness, $F\left(R^{2}\right)=F(\bar{R})$ and we are done. If $p\left(R_{j}^{2}\right) \neq p\left(\bar{R}_{j}\right)$. Then, according to the reasoning described below, repeat the leapfrogging steps described above $\nu \in \mathbb{N}^{+}$amount of times.

Recall that $\delta_{1}=\frac{3}{2} \delta_{0}$ and $\delta_{2}=\frac{3}{2} \delta_{1}$. Hence, $\delta_{\nu}=\frac{3}{2} \delta_{\nu-1}=\left(\frac{3}{2}\right)^{\nu} \delta_{0}$ and since $\delta_{0} \neq 0$, in the limit, $\lim _{\nu \rightarrow \infty} \delta_{\nu}=\infty$. Thus, for each profile $\bar{R} \in \mathcal{R}^{N}$ such that $\bar{R}_{-j}=R_{-j}$ and $p\left(\bar{R}_{j}\right)>p\left(R_{j}\right)$, there exists a finite $\nu \in \mathbb{N}^{+}$such that the distance $\delta_{\nu}>\left|\underline{F}(R)-p\left(\bar{R}_{j}\right)\right|$. Therefore, for each profile $\bar{R} \in \mathcal{R}^{N}$ such that $\bar{R}_{-j}=R_{-j}$ and $p\left(\bar{R}_{j}\right)>p\left(R_{j}\right)$, there exists a profile $R^{\nu}$ such that $\operatorname{Conv}\left(R^{\nu}\right)=\operatorname{Conv}(p(\bar{R}))$ and the following holds. If minimum $\underline{F}(R)<\bar{p}(R)=p\left(R_{n}\right)<p\left(\bar{R}_{j}\right)$, then minima $\underline{F}\left(R^{\nu}\right)=\underline{F}(R)$ and moreover, if also maximum $\bar{F}(R)<\bar{p}(R)$, then $F\left(R^{\nu}\right)=F(R)$. Since $\operatorname{Conv}\left(R^{\nu}\right)=\operatorname{Conv}(p(\bar{R}))$, by extreme-peaks-onliness, $F\left(R^{\nu}\right)=F(\bar{R})$ and we are done.

Case 2. Let agent $j=n$ have the unique largest peak at profiles $R$ and $\bar{R}$. Let agent $k \in N \backslash\{j\}$ be a middle agent at profile $R$ and construct profile $R^{1}$ by changing his preferences to $R_{k}^{1}$ such that his peak $p\left(R_{k}^{1}\right)=\bar{p}(R)$, i.e., $R^{1}=$ $\left(R_{-k}, R_{k}^{1}\right)$. Since $\operatorname{Conv}\left(R^{1}\right)=\operatorname{Conv}(p(R))$, by extreme-peaks-onliness, $F\left(R^{1}\right)=$ $F(R)$. Therefore, since minimum $\underline{F}(R)<\bar{p}(R)=\bar{p}\left(R^{1}\right)=p\left(R_{k}^{1}\right)<p\left(\bar{R}_{j}\right)$, by Case 1, minima $\underline{F}(\bar{R})=\underline{F}\left(R^{1}\right)=\underline{F}(R)$ and moreover, if also maximum $\bar{F}(R)<$ $\bar{p}(R)=\bar{p}\left(R^{1}\right)$, by Case $1, F(\bar{R})=F\left(R^{1}\right)=F(R)$.

Lemma 2.10 (Efficiency and one-sided replacement-dominance $\Rightarrow$ uncompromisingness). If a fixed population consists of at least 3 agents, then an associated fp-choice correspondence that satisfies efficiency and one-sided replacement-dominance also satisfies uncompromisingness.

Proof. Let fixed population $N \in \mathcal{P}$ be such that $|N| \geq 3$ and fp-choice correspondence $F \in \mathcal{F}^{N}$ satisfy efficiency and one-sided replacement-dominance. By Lemmas 2.5 (Appendix 2.B) and 2.7, $F$ satisfies extreme-peaks-onliness and peakmonotonicity. Let agent $j \in N$ and the pair of profiles $R, \bar{R} \in \mathcal{R}^{N}$ be such that $R_{-j}=\bar{R}_{-j}$. In the following, we refer to agents who have neither the unique 
smallest peak nor the unique largest peak as middle agents.

(i) We show that if $\left[p\left(R_{j}\right)<\underline{F}(R)\right.$ and $\left.p\left(\bar{R}_{j}\right) \leq \underline{F}(R)\right]$ or $\left[p\left(R_{j}\right)>\underline{F}(R)\right.$ and $p\left(\bar{R}_{j}\right) \geq \underline{F}(R)$ ], then minima $\underline{F}(R)=\underline{F}(\bar{R})$. By efficiency, $F(R) \in \operatorname{PE}(R)$. Hence by Proposition 2.1 (i), $F(R) \subseteq \operatorname{Conv}(p(R))$. Notice that $\operatorname{Conv}(p(\bar{R})) \subseteq$ $\operatorname{Conv}(p(R))$ or $\operatorname{Conv}(p(\bar{R})) \supseteq \operatorname{Conv}(p(R))$.

Case 1. Let $p\left(R_{j}\right)<\underline{F}(R)$ and $p\left(\bar{R}_{j}\right) \leq \underline{F}(R)$. Hence, since $F(R) \subseteq$ $\operatorname{Conv}(p(R)), p\left(R_{j}\right) \neq \bar{p}(R)$.

Case 1.1. Let $\operatorname{Conv}(p(\bar{R}))=\operatorname{Conv}(p(R))$. By extreme-peaks-onliness, $F(R)=$ $F(\bar{R})$.

Case 1.2. Let $\operatorname{Conv}(p(\bar{R})) \subsetneq \operatorname{Conv}(p(R))$. Hence, agent $j$ has the unique smallest peak at profile $R$ and minimum $\underline{F}(R) \geq p\left(\bar{R}_{j}\right) \geq p(\bar{R})>p\left(R_{j}\right)$. Begin from profile $R$ and construct profile $\bar{R}$ by changing agent $j$ 's preferences to $\bar{R}_{j}$, i.e., $\bar{R}=\left(R_{-j}, \bar{R}_{j}\right)$. Since $p\left(\bar{R}_{j}\right)>p\left(R_{j}\right)$ and $\bar{R}_{-j}=R_{-j}$, by peak-monotonicity, minimum $\underline{F}(\bar{R}) \geq \underline{F}(R)$. If minimum $\underline{F}(\bar{R})>\underline{F}(R) \geq p\left(\bar{R}_{j}\right)$, then $F(\bar{R}) \neq F(R)$ and minimum $\underline{F}(\bar{R})>\underline{p}(\bar{R})>p\left(R_{j}\right)$. Since $\bar{R}_{-j}=R_{-j}$, by Lemma 2.9 (ii) (with the roles of $R$ and $\bar{R}$ reversed), $F(\bar{R})=F(R) \neq F(\bar{R})$, a contradiction. Therefore, minima $\underline{F}(\bar{R})=\underline{F}(R)$.

Case 1.3. Let $\operatorname{Conv}(p(\bar{R})) \supsetneq \operatorname{Conv}(p(R))$. Hence, agent $j$ has the unique smallest peak at profile $\bar{R}$ and minimum $\underline{F}(R)>p\left(R_{j}\right) \geq p(R)>p\left(\bar{R}_{j}\right)$. By Lemma 2.9 (ii), $F(\bar{R})=F(R)$.

Case 2. Let $p\left(R_{j}\right)>\underline{F}(R)$ and $p\left(\bar{R}_{j}\right) \geq \underline{F}(R)$. Hence, since $F(R) \subseteq$ $\operatorname{Conv}(p(R)), p\left(R_{j}\right) \neq \underline{p}(R)$.

Case 2.1. Let $\operatorname{Conv}(p(\bar{R}))=\operatorname{Conv}(p(R))$. By extreme-peaks-onliness, $F(R)=$ $F(\bar{R})$.

Case 2.2. Let $\operatorname{Conv}(p(\bar{R})) \subsetneq \operatorname{Conv}(p(R))$. Hence, agent $j$ has the unique largest peak at profile $R$ and minimum $\underline{F}(R) \leq p\left(\bar{R}_{j}\right) \leq \bar{p}(\bar{R})<p\left(R_{j}\right)$. Begin from profile $R$ and construct profile $\bar{R}$ by changing agent $j$ 's preferences to $\bar{R}_{j}$, i.e., $\bar{R}=\left(R_{-j}, \bar{R}_{j}\right)$. Since $p\left(\bar{R}_{j}\right)<p\left(R_{j}\right)$ and $\bar{R}_{-j}=R_{-j}$, by peak-monotonicity, minimum $\underline{F}(\bar{R}) \leq \underline{F}(R)$. If minimum $\underline{F}(\bar{R})<\underline{F}(R) \leq p\left(\bar{R}_{j}\right)$, then minimum $\underline{F}(\bar{R})<\bar{p}(\bar{R})<p\left(R_{j}\right)$. Since $\bar{R}_{-j}=R_{-j}$, by Lemma 2.9 (i) (with the roles of $R$ and $\bar{R}$ reversed), minimum $\underline{F}(\bar{R})=\underline{F}(R) \neq \underline{F}(\bar{R})$, a contradiction. Therefore, minima $\underline{F}(\bar{R})=\underline{F}(R)$.

Case 2.3. Let $\operatorname{Conv}(p(\bar{R})) \supsetneq \operatorname{Conv}(p(R))$. Hence, agent $j$ has the unique largest peak at profile $\bar{R}$ and minimum $\underline{F}(R)<p\left(R_{j}\right) \leq \bar{p}(R)<p\left(\bar{R}_{j}\right)$. By Lemma 2.9 (i), minima $\underline{F}(\bar{R})=\underline{F}(R)$.

(ii) The proof that if $\left[p\left(R_{j}\right)>\bar{F}(R)\right.$ and $\left.p\left(\bar{R}_{j}\right) \geq \bar{F}(R)\right]$ or $\left[p\left(R_{j}\right)<\bar{F}(R)\right.$ and $\left.p\left(\bar{R}_{j}\right) \leq \bar{F}(R)\right]$, then maxima $\bar{F}(R)=\bar{F}(\bar{R})$ is symmetric to the proof of 
(i).

The next result is crucial in the proof of Theorem 2.1.

Lemma 2.11. Let fixed population $N \in \mathcal{P}$ be such that $|N| \geq 3$ and fp-choice correspondence $F \in \mathcal{F}^{N}$ satisfy efficiency and one-sided replacement-dominance. Let fp-target set correspondence $F^{a, b} \in \mathcal{F}^{N}$. For each pair of profiles $R, \bar{R} \in \mathcal{R}^{N}$ such that $\operatorname{Conv}(p(\bar{R})) \subseteq \operatorname{Conv}(p(R))$, if $F(R)=F^{a, b}(R)$, then $F(\bar{R})=F^{a, b}(\bar{R})$.

Proof. Let fixed population $N \in \mathcal{P}$ be such that $|N| \geq 3$ and fp-choice correspondence $F \in \mathcal{F}^{N}$ satisfy efficiency and one-sided replacement-dominance. Let fp-target set correspondence $F^{a, b} \in \mathcal{F}^{N}$. By Propositions 2.1 and 2.4, $F^{a, b}$ satisfies efficiency and one-sided replacement-dominance. By Lemma 2.5 (Appendix 2.B), Lemma 2.10, and Lemma 2.8, $F$ and $F^{a, b}$ satisfy extreme-peaks-onliness, uncompromisingness, and set-uncompromisingness.

Let the pair of profiles $R, \bar{R} \in \mathcal{R}^{N}$ be such that $F(R)=F^{a, b}(R)$ and $\operatorname{Conv}(p(\bar{R})) \subseteq \operatorname{Conv}(p(R))$. Without loss of generality, assume that $N=$ $\{1, \ldots, n\}$ and $\underline{p}(R)=p\left(R_{1}\right) \leq \cdots \leq p\left(R_{n}\right)=\bar{p}(R)$. We show that $F(\bar{R})=$ $F^{a, b}(\bar{R})$.

Case 1. Let $\operatorname{Conv}(p(\bar{R}))=\operatorname{Conv}(p(R))$. By extreme-peaks-onliness and the definition of $F^{a, b}, F(\bar{R})=F(R)=F^{a, b}(R)=F^{a, b}(\bar{R})$.

Case 2. Let $\operatorname{Conv}(p(\bar{R})) \subsetneq \operatorname{Conv}(p(R))$ be such that $\underline{p}(\bar{R})>\underline{p}(R)$ and $\bar{p}(\bar{R})=$ $\bar{p}(R)$. By extreme-peaks-onliness, it is without loss of generality to assume that at both profiles $R$ and $\bar{R}$, agent 1 has the smallest peak and all other agents have the largest peak, i.e., $R=\left(R_{1}, R_{n}, \ldots, R_{n}\right)$ such that $p\left(R_{1}\right) \leq p\left(R_{n}\right)$ and $\bar{R}=\left(\bar{R}_{1}, R_{n}, \ldots, R_{n}\right)$ such that $p\left(\bar{R}_{1}\right) \leq p\left(R_{n}\right)$. Hence, $R_{-1}=\bar{R}_{-1}$ and $\underline{p}(R)<$ $\underline{p}(\bar{R}) \leq \bar{p}(\bar{R})=\bar{p}(R)$. By efficiency and Proposition 2.1 (i), $p\left(R_{1}\right)=\underline{p}(R) \leq$ $\underline{F}(R) \leq \bar{F}(R) \leq \bar{p}(R)$ and $p\left(\bar{R}_{1}\right)=\underline{p}(\bar{R}) \leq \underline{F}(\bar{R}) \leq \bar{F}(\bar{R}) \leq \bar{p}(\bar{R})$.

Case 2.1. Recall that $\operatorname{Conv}(p(\bar{R})) \subsetneq \operatorname{Conv}(p(R))$ is such that $[\underline{p}(\bar{R})>\underline{p}(R)$ and $\bar{p}(\bar{R})=\bar{p}(R)]$ and in addition, let $p\left(\bar{R}_{1}\right)=\underline{p}(\bar{R}) \leq \underline{F}(R)$. Then, $p\left(R_{1}\right)=$ $\underline{p}(R)<\underline{F}(R)$. By set-uncompromisingness, $F(\bar{R})=F(R)=F^{a, b}(R)$ and by the definition of $F^{a, b}$, point $a \geq p(\bar{R})$. If point $a \leq \bar{p}(R)=\bar{p}(\bar{R})$, then $F^{a, b}(R)=$ $[a, b] \cap \operatorname{Conv}(p(R))=[a, b] \cap \operatorname{Conv}(p(\bar{R}))=F^{a, b}(\bar{R})$. If point $a>\bar{p}(R)=\bar{p}(\bar{R})$, then, $F^{a, b}(R)=\{\bar{p}(R)\}=F^{a, b}(\bar{R})$. Therefore, $F(\bar{R})=F^{a, b}(\bar{R})$.

Case 2.2. Recall that $\operatorname{Conv}(p(\bar{R})) \subsetneq \operatorname{Conv}(p(R))$ is such that $[p(\bar{R})>p(R)$ and $\bar{p}(\bar{R})=\bar{p}(R)]$ and in addition, let $p\left(\bar{R}_{1}\right)=\underline{p}(\bar{R})>\underline{F}(R)$ and $p\left(\bar{R}_{1}\right)=\underline{p}(R) \leq$ $\bar{F}(R)$. Then, $\underline{F}(R) \neq \bar{F}(R)$ and $p\left(R_{1}\right)<\bar{F}(R)$. By uncompromisingness, maxima $\bar{F}(\bar{R})=\bar{F}(R)$. Recall that by efficiency and Proposition 2.1 (i), minimum $\underline{F}(\bar{R}) \geq$ $\underline{p}(\bar{R})=p\left(\bar{R}_{1}\right)$. Next, assuming that minimum $\underline{F}(\bar{R})>\underline{p}(\bar{R})=p\left(\bar{R}_{1}\right)>\underline{F}(R)$ 
results in a contradiction as follows: since $p\left(\bar{R}_{1}\right)<\underline{F}(\bar{R})$ and $p\left(R_{1}\right)<\underline{F}(\bar{R})$, by uncompromisingness, minimum $\underline{F}(R)=\underline{F}(\bar{R}) \neq \underline{F}(R)$, a contradiction. Hence, minimum $\underline{F}(\bar{R})=\underline{p}(\bar{R})$ and thus, $F(\bar{R})=[\underline{p}(\bar{R}), \bar{F}(R)]$. Since $\operatorname{Conv}(p(\bar{R})) \subsetneq$ $\operatorname{Conv}(p(R))$ and $F(R)=[a, b] \cap \operatorname{Conv}(p(R)), F(\bar{R})=F(R) \cap \operatorname{Conv}(p(\bar{R}))=[a, b] \cap$ $\operatorname{Conv}(p(\bar{R}))$. Therefore, by the definition of $F^{a, b}, F(\bar{R})=[a, b] \cap \operatorname{Conv}(p(\bar{R}))=$ $F^{a, b}(\bar{R})$.

Case 2.3. Recall that $\operatorname{Conv}(p(\bar{R})) \subsetneq \operatorname{Conv}(p(R))$ is such that $[\underline{p}(\bar{R})>\underline{p}(R)$ and $\bar{p}(\bar{R})=\bar{p}(R)]$ and in addition, let $p\left(\bar{R}_{1}\right)=\underline{p}(\bar{R})>\bar{F}(R) \geq \underline{F}(R)$. By the definition of $F^{a, b}$, points $a, b<p(\bar{R})$. Next, assuming that maximum $\bar{F}(\bar{R})>$ $p(\bar{R})=p\left(\bar{R}_{1}\right)>\bar{F}(R)$ results in a contradiction as follows: since $p\left(\bar{R}_{1}\right)<\bar{F}(\bar{R})$ and $p\left(R_{1}\right)<\bar{F}(\bar{R})$, by uncompromisingness, maximum $\bar{F}(R)=\bar{F}(\bar{R}) \neq \bar{F}(R)$, a contradiction. Hence, maximum $\bar{F}(\bar{R})=\underline{p}(\bar{R})$ and thus $F(\bar{R})=\{\underline{p}(\bar{R})\}$. Since point $b<\underline{p}(\bar{R})$, by the definition of $F^{a, b}, F(\bar{R})=\{\underline{p}(\bar{R})\}=F^{a, b}(\bar{R})$.

Case 3. Let $\operatorname{Conv}(p(\bar{R})) \subsetneq \operatorname{Conv}(p(R))$ be such that $\underline{p}(\bar{R})=\underline{p}(R)$ and $\bar{p}(\bar{R})<$ $\bar{p}(R)$. By a symmetric proof to Case $2, F(\bar{R})=F^{a, b}(\bar{R})$.

Case 4. Let $\operatorname{Conv}(p(\bar{R})) \subsetneq \operatorname{Conv}(p(R))$ be such that $p(\bar{R})>p(R)$ and $\bar{p}(\bar{R})<$ $\bar{p}(R)$. Let profile $R^{1} \in \mathcal{R}^{N}$ be such that $\underline{p}\left(R^{1}\right)=\underline{p}(\bar{R})>\underline{p}(R)$ and $\bar{p}\left(R^{1}\right)=\bar{p}(R)$. By Case $2, F\left(R^{1}\right)=F^{a, b}\left(R^{1}\right)$. Next, since $\underline{p}(\bar{R})=\underline{p}\left(R^{1}\right)$ and $\bar{p}(\bar{R})<\bar{p}\left(R^{1}\right)$, by Case $3, F(\bar{R})=F^{a, b}(\bar{R})$.

Proof of Theorem 2.1. If part. By Propositions 2.1 and 2.4, each fp-target set correspondence satisfies efficiency and one-sided replacement-dominance.

Only if part. Let fixed population $N \in \mathcal{P}$ be such that $|N| \geq 3$ and fp-choice correspondence $F \in \mathcal{F}^{N}$ satisfy efficiency and one-sided replacement-dominance. By Lemma 2.5 (Appendix 2.B), Lemma 2.10, and Lemma 2.8, F satisfies extremepeaks-onliness, uncompromisingness, and set-uncompromisingness.

For each pair of points $\alpha, \beta \in \mathbb{R}$ such that $\alpha \leq \beta$, define a profile $R^{\alpha, \beta} \in \mathcal{R}^{N}$ to be such that $p\left(R^{\alpha, \beta}\right)=\alpha$ and $\bar{p}\left(R^{\alpha, \beta}\right)=\beta$. Without loss of generality, assume that $N=\{1, \ldots, n\}$ and $\alpha=p\left(R_{1}^{\alpha, \beta}\right) \leq \ldots \leq p\left(R_{n}^{\alpha, \beta}\right)=\beta$. By efficiency and Proposition 2.1 (i), $\alpha \leq \underline{F}\left(R^{\alpha, \beta}\right) \leq \bar{F}\left(R^{\alpha, \beta}\right) \leq \beta$.

We prove that there exists an fp-target set correspondence $F^{a, b} \in \mathcal{F}^{N}$ such that for each profile $R \in \mathcal{R}^{N}, F(R)=F^{a, b}(R)$.

There are four cases. Loosely speaking, in all but the last case the proof proceeds as follows. Given a profile $R^{\alpha, \beta} \in \mathcal{R}^{N}$ and for each profile $R \in \mathcal{R}^{N}$ we select a profile such that the convex hull of its peaks is a superset of both $\operatorname{Conv}\left(R^{\alpha, \beta}\right)$ and $\operatorname{Conv}(p(R))$ and then, we apply Lemma 2.11 to show that $F(R)=$ $F^{a, b}(R)$. 
Case 1. There exist $\alpha, \beta \in \mathbb{R}$ such that for $R^{\alpha, \beta} \in \mathcal{R}^{N}, \alpha<\underline{F}\left(R^{\alpha, \beta}\right) \leq$ $\bar{F}\left(R^{\alpha, \beta}\right)<\beta$. Define points $a:=\underline{F}\left(R^{\alpha, \beta}\right)$ and $b:=\bar{F}\left(R^{\alpha, \beta}\right)$. Since $F\left(R^{\alpha, \beta}\right)=$ $[a, b]=[a, b] \cap \operatorname{Conv}\left(R^{\alpha, \beta}\right)$, by the definition of $F^{a, b}, F\left(R^{\alpha, \beta}\right)=F^{a, b}\left(R^{\alpha, \beta}\right)$. Let $R \in \mathcal{R}^{N}$. Begin from profile $R^{\alpha, \beta}$ and construct profile $R^{1}$ by changing agent 1 's preferences to $R_{1}^{1}$ such that his peak

$$
p\left(R_{1}^{1}\right)= \begin{cases}p\left(R_{1}^{\alpha, \beta}\right) & \text { if } p\left(R_{1}^{\alpha, \beta}\right) \leq \underline{p}(R) \\ \underline{p}(R) & \text { otherwise, }\end{cases}
$$

i.e., $R^{1}=\left(R_{-1}^{\alpha, \beta}, R_{1}^{1}\right)$. Since $p\left(R_{1}^{\alpha, \beta}\right)<\underline{F}\left(R^{\alpha, \beta}\right)$ and $p\left(R_{1}^{1}\right)<\underline{F}\left(R^{\alpha, \beta}\right)$, by setuncompromisingness, $F\left(R^{1}\right)=F\left(R^{\alpha, \beta}\right)=[a, b]$. Then, change agent $n$ 's preferences to $R_{n}^{2}$ such that his peak

$$
p\left(R_{n}^{2}\right)= \begin{cases}p\left(R_{n}^{1}\right) & \text { if } p\left(R_{n}^{1}\right) \geq \bar{p}(R) \\ \bar{p}(R) & \text { otherwise }\end{cases}
$$

i.e., $R^{2}=\left(R_{-n}^{1}, R_{n}^{2}\right)$. Since $p\left(R_{n}^{1}\right)>\bar{F}\left(R^{1}\right)$ and $p\left(R_{n}^{2}\right)>\bar{F}\left(R^{1}\right)$, by setuncompromisingness, $F\left(R^{2}\right)=F\left(R^{1}\right)=[a, b]$. Since $F\left(R^{2}\right)=[a, b]=[a, b] \cap$ $\operatorname{Conv}\left(R^{2}\right)$, by the definition of $F^{a, b}, F\left(R^{2}\right)=F^{a, b}\left(R^{2}\right)$. Since, $F\left(R^{2}\right)=F^{a, b}\left(R^{2}\right)$ and $\operatorname{Conv}(p(R)) \subseteq \operatorname{Conv}\left(R^{2}\right)$, by Lemma 2.11, $F(R)=F^{a, b}(R)$.

Case 2. There exist $\alpha, \beta \in \mathbb{R}$ such that for $R^{\alpha, \beta} \in \mathcal{R}^{N}, \alpha=\underline{F}\left(R^{\alpha, \beta}\right) \leq$ $\bar{F}\left(R^{\alpha, \beta}\right)<\beta$, and for each $\bar{\alpha} \leq \alpha$ and its associated $R^{\bar{\alpha}, \beta} \in \mathcal{R}^{N}, \bar{\alpha}=\underline{F}\left(R^{\bar{\alpha}, \beta}\right) \leq$ $\bar{F}\left(R^{\bar{\alpha}, \beta}\right)<\beta$.

Case 2.1. There exist $\alpha, \beta \in \mathbb{R}$ as specified in Case 2 and in addition, $\alpha=$ $\underline{F}\left(R^{\alpha, \beta}\right)<\bar{F}\left(R^{\alpha, \beta}\right)<\beta$. Define points $a:=-\infty$ and $b:=\bar{F}\left(R^{\alpha, \beta}\right)$. Since $F\left(R^{\alpha, \beta}\right)=\left[\underline{p}\left(R^{\alpha, \beta}\right), b\right]=[a, b] \cap \operatorname{Conv}\left(R^{\alpha, \beta}\right)$, by the definition of $F^{a, b}, F\left(R^{\alpha, \beta}\right)=$ $F^{a, b}\left(R^{\alpha, \beta}\right)$. Let $R \in \mathcal{R}^{N}$. Begin from profile $R^{\alpha, \beta}$ and construct profile $R^{1}$ by changing agent 1 's preferences to $R_{1}^{1}$ such that his peak

$$
p\left(R_{1}^{1}\right)= \begin{cases}p\left(R_{1}^{\alpha, \beta}\right) & \text { if } p\left(R_{1}^{\alpha, \beta}\right) \leq \underline{p}(R) \\ \underline{p}(R) & \text { otherwise, }\end{cases}
$$

i.e., $R^{1}=\left(R_{-1}^{\alpha, \beta}, R_{1}^{1}\right)$. Since $\underline{p}\left(R^{1}\right) \leq \alpha$ and $\bar{p}\left(R^{1}\right)=\beta$, as specified in Case 2 and by extreme-peaks-onliness, $\underline{p}\left(R^{1}\right)=\underline{F}\left(R^{1}\right)$. Since $p\left(R_{1}^{\alpha, \beta}\right)<\bar{F}\left(R^{\alpha, \beta}\right)$ and $p\left(R_{1}^{1}\right)<\bar{F}\left(R^{\alpha, \beta}\right)$, by uncompromisingness, maxima $\bar{F}\left(R^{1}\right)=\bar{F}\left(R^{\alpha, \beta}\right)=b$. Hence, 
$F\left(R^{1}\right)=\left[\underline{p}\left(R^{1}\right), b\right]$. Then, change agent $n$ 's preferences to $R_{n}^{2}$ such that his peak

$$
p\left(R_{n}^{2}\right)= \begin{cases}p\left(R_{n}^{1}\right) & \text { if } p\left(R_{n}^{1}\right) \geq \bar{p}(R) \\ \bar{p}(R) & \text { otherwise }\end{cases}
$$

i.e., $R^{2}=\left(R_{-n}^{1}, R_{n}^{2}\right)$. Since $p\left(R_{n}^{1}\right)>\bar{F}\left(R^{1}\right)$ and $p\left(R_{n}^{2}\right)>\bar{F}\left(R^{1}\right)$, by setuncompromisingness, $F\left(R^{2}\right)=F\left(R^{1}\right)=\left[\underline{p}\left(R^{2}\right), b\right]$. Since $F\left(R^{2}\right)=\left[\underline{p}\left(R^{2}\right), b\right]=$ $[a, b] \cap \operatorname{Conv}\left(R^{2}\right)$, by the definition of $F^{a, b}, F\left(R^{2}\right)=F^{a, b}\left(R^{2}\right)$. Since $F\left(R^{2}\right)=$ $F^{a, b}\left(R^{2}\right)$ and $\operatorname{Conv}(p(R)) \subseteq \operatorname{Conv}\left(R^{2}\right)$, by Lemma 2.11, $F(R)=F^{a, b}(R)$.

Case 2.2. There exist $\alpha, \beta \in \mathbb{R}$, as specified in Case 2 and in addition, $\alpha=$ $\underline{F}\left(R^{\alpha, \beta}\right)=\bar{F}\left(R^{\alpha, \beta}\right)<\beta$, and for each $\bar{\alpha} \leq \alpha$ and its associated $R^{\bar{\alpha}, \beta} \in \mathcal{R}^{N}$, $\bar{\alpha}=\underline{F}\left(R^{\bar{\alpha}, \beta}\right)=\bar{F}\left(R^{\bar{\alpha}, \beta}\right)<\beta$. Define points $a, b:=-\infty$. Since $b<\underline{p}\left(R^{\alpha, \beta}\right)$ and $F\left(R^{\alpha, \beta}\right)=\left\{\underline{p}\left(R^{\alpha, \beta}\right)\right\}$, by the definition of $F^{a, b}, F\left(R^{\alpha, \beta}\right)=F^{a, b}\left(R^{\alpha, \beta}\right)$. Let $R \in \mathcal{R}^{N}$. Begin from profile $R^{\alpha, \beta}$ and construct profile $R^{1}$ by changing agent 1 's preferences to $R_{1}^{1}$ such that his peak

$$
p\left(R_{1}^{1}\right)= \begin{cases}p\left(R_{1}^{\alpha, \beta}\right) & \text { if } p\left(R_{1}^{\alpha, \beta}\right) \leq \underline{p}(R) \\ \underline{p}(R) & \text { otherwise, }\end{cases}
$$

i.e., $R^{1}=\left(R_{-1}^{\alpha, \beta}, R_{1}^{1}\right)$. Since $\underline{p}\left(R^{1}\right) \leq \alpha$ and $\bar{p}\left(R^{1}\right)=\beta$, as specified in this case and by extreme-peaks-onliness, $F\left(R^{1}\right)=\left\{\underline{p}\left(R^{1}\right)\right\}$. Then, change agent $n$ 's preferences to $R_{n}^{2}$ such that his peak

$$
p\left(R_{n}^{2}\right)= \begin{cases}p\left(R_{n}^{1}\right) & \text { if } p\left(R_{n}^{1}\right) \geq \bar{p}(R) \\ \bar{p}(R) & \text { otherwise }\end{cases}
$$

i.e., $R^{2}=\left(R_{-n}^{1}, R_{n}^{2}\right)$. Since $p\left(R_{n}^{1}\right)>\bar{F}\left(R^{1}\right)$ and $p\left(R_{n}^{2}\right)>\bar{F}\left(R^{1}\right)$, by setuncompromisingness, $F\left(R^{2}\right)=F\left(R^{1}\right)=\left\{\underline{p}\left(R^{2}\right)\right\}$. Since $b<\underline{p}\left(R^{2}\right)$, by the definition of $F^{a, b}, F\left(R^{2}\right)=F^{a, b}\left(R^{2}\right)$. Since $F\left(R^{2}\right)=F^{a, b}\left(R^{2}\right)$ and $\operatorname{Conv}(p(R)) \subseteq$ $\operatorname{Conv}\left(R^{2}\right)$, by Lemma $2.11, F(R)=F^{a, b}(R)$.

Case 3. There exist $\alpha, \beta \in \mathbb{R}$ such that for $R^{\alpha, \beta} \in \mathcal{R}^{N}, \alpha<\underline{F}\left(R^{\alpha, \beta}\right) \leq$ $\bar{F}\left(R^{\alpha, \beta}\right)=\beta$, and for each $\bar{\beta} \geq \beta$ and its associated $R^{\alpha, \bar{\beta}} \in \mathcal{R}^{N}, \alpha<\underline{F}\left(R^{\alpha, \bar{\beta}}\right) \leq$ $\bar{F}\left(R^{\alpha, \bar{\beta}}\right)=\bar{\beta}$. The proof of this case is symmetric to Case 2 .

Case 4. For each $\alpha, \beta \in \mathbb{R}$ such that $\alpha \leq \beta$ and its associated $R^{\alpha, \beta} \in \mathcal{R}^{N}$, $\alpha=\underline{F}\left(R^{\alpha, \beta}\right) \leq \bar{F}\left(R^{\alpha, \beta}\right)=\beta$. Define points $a:=-\infty$ and $b:=\infty$. Since for each $\alpha, \beta \in \mathbb{R}$ and its associated $R^{\alpha, \beta} \in \mathcal{R}^{N}, \alpha=\underline{F}\left(R^{\alpha, \beta}\right) \leq \bar{F}\left(R^{\alpha, \beta}\right)=\beta$, by extreme-peaks-onliness, for each $R \in \mathcal{R}^{N}, F(R)=\operatorname{Conv}(p(R))$. Therefore, since 
$a<\underline{p}(R)$ and $b>\bar{p}(R)$, by the definition of $F^{a, b}, F(R)=F^{a, b}(R)$. 


\title{
Chapter 3
}

\section{On strategy-proofness and single- peakedness: median-voting over intervals}

\begin{abstract}
We study correspondences that choose an interval of alternatives when agents have single-peaked preferences. Similar to Klaus and Storcken (2002), we ordinally extend these preferences over intervals. Loosely speaking, we extend the results of Moulin (1980) to our setting and show that the results of Ching (1997) cannot always be similarly extended. Our main results are the following. First, strategy-proofness and peaks-onliness characterize the class of generalized median correspondences. Second, we characterize the anonymous sub-class of generalized median correspondences, the class of median correspondences. Third, although peaks-onliness cannot be replaced by the "weaker" property of continuity in the aforementioned results -as is the case in Ching (1997)this equivalence is achieved when voter-sovereignty is also required, in the characterizations of the classes of efficient generalized median correspondences and efficient median correspondences. Finally, when preferences are symmetric and single-peaked, only the characterizations for the classes of efficient generalized median correspondences and efficient median correspondences can be extended; moreover, in these results the properties of peaks-onliness and continuity are unnecessary.
\end{abstract}

\subsection{Introduction}

We study the problem where an interval of alternatives is chosen on the interval $[0,1]$ based on the preferences of a finite number of agents. This interval can be considered as the political spectrum, while the chosen interval can in turn be considered as the legislative constitution or the governmental coalition (in the sense that some "extreme" views are not accounted for by the constitution or are not represented by any member(s) of the governmental coalition). We assume 
that agents have single-peaked preferences defined over all alternatives on $[0,1]$; that is, an agent's welfare is strictly increasing up to his "peak" (his favorite alternative), and is strictly decreasing thereafter. These agents, when comparing two intervals, only consider their best (most favorite) alternative and their worst (least favorite) alternative(s) on each interval. Moreover, we look into the situation where the voting mechanism choosing the interval of alternatives guarantees that the agents announce their true preferences; in other words, we are interested in voting mechanisms -which we call (choice) correspondences- that are strategy-proof.

Although the classic result of Gibbard and Satterthwaite establishes that on the full domain of preferences -with more than two alternatives available- strategyproofness and non-dictatorship are incompatible (Gibbard, 1973; Satterthwaite, 1975), this is not true for the domain of single-peaked preferences, the domain of interest in this paper.

This compatibility between the two aforementioned properties has been well studied in the context of (choice) functions and for infinite sets of alternatives, where following the announcement of the agents' (single-peaked) preferences one alternative is chosen. Specifically, it has been shown that strategy-proofness and peaks-onliness (the agents only announce their peak) characterize the class of generalized median rules (described in Section 3.3.1) (Moulin, 1980). Moreover, when also requiring the property of either efficiency (in the Pareto sense), or anonymity (the names of the agents don't matter), or both to be satisfied, the sub-classes of either efficient generalized median rules (Section 3.3.1), or median rules (Section 3.3.2), or efficient median rules (Section 3.3.2) are characterized (Moulin, 1980). A similar result also holds for the one-dimensional case, when the range of the function is closed and not connected (Barberà and Jackson, 1994). In addition, on the smaller domain of quadratic and separable preferences ${ }^{1}$ and on dimensions equal or larger to 1 , peaks-onliness can be substituted by unanimity (when a common best alternative exists, it is chosen) (Border and Jordan, 1983); furthermore, it turns out that in these results two of the required properties can be weakened; specifically, peak-onliness and efficiency can be substituted by continuity (a small change in the announced preferences does not change the outcome a lot) and voter-sovereignty (no alternative is a priori excluded from being chosen) respectively (Ching, 1997). ${ }^{2}$ Finally, a measure of manipulability was recently

\footnotetext{
${ }^{1}$ In this domain, an agent's welfare depends on the distance of the alternative chosen from his peak, projected in every dimension. Specifically, the larger the sum of all such projected distances, the smaller the welfare gained.

${ }^{2}$ Although technically continuity is not weaker than peaks-onliness, loosely speaking, it imposes fewer restrictions on the result.
} 
proposed, that can be used to compare two generalized median rules (via some necessary and sufficient conditions) (Arribillaga and Massó, 2016).

For the case where a single alternative is chosen among a finite set (of alternatives), strategy-proofness and voter-sovereignty characterize, on the domain of strict preferences, a class of functions similar to the class of efficient generalized median rules (Barberà et al., 1993). Moreover, the admissible preferences of all agents being top-connected ${ }^{3}$ characterize the maximal domain in which (i) every strategy-proof and unanimous function is a generalized median rule, and (ii) every generalized median rule is strategy-proof (Achuthankutty and Roy, 2017).

When departing from the setting where agents have single-peaked preferences and only one alternative is chosen, a few more results should be mentioned. First, in the case of probabilistic functions, ${ }^{4}$ where the agents' single-peaked preferences are ordinarily extended over probability distributions via first-order stochastic dominance, similar results to Moulin's results (1980) were achieved (Ehlers et al., 2002). Next, if agents have single-dipped preferences, ${ }^{5}$ strategy-proofness and unanimity characterize the class of collections of 0 -decisive sets with a tie-breaker ${ }^{6}$ (Manjunath, 2014). Last but not least, we must mention the two following results where the agents' preferences are single-peaked and two alternatives can be chosen, with the agents comparing different pairs of alternatives using the max-extension. ${ }^{7}$

(i) Strategy-proofness, continuity, anonymity, and users-only ${ }^{8}$ characterize the class of double median functions ${ }^{9}$ (Heo, 2013).

(ii) Efficiency and replacement-dominance ${ }^{10}$ characterize the class of rules

\footnotetext{
${ }^{3}$ For every agent and every pair of "neighboring" alternatives $(a, b)$ there exist admissible preferences such that $a$ is the most favorite alternative and $b$ is the second most favorite alternative.

${ }^{4}$ Given the agents' preferences, a probability distribution over all alternatives is chosen.

"An agent's welfare is strictly decreasing up to his "dip" (his least favorite alternative), and is strictly increasing thereafter.

${ }^{6}$ Each such function chooses either the minimum or the maximum alternative. Loosely speaking, if all agents are indifferent between the two alternatives the choice depends on the preference profile (over all other alternatives). Otherwise, the choice depends on the number of agents preferring the minimum over the maximum alternative, their identities, and their preferences.

${ }^{7}$ When comparing two pairs of alternatives $X=\left\{x_{1}, x_{2}\right\}$ and $Y=\left\{y_{1}, y_{2}\right\}$, an agent first locates in each pair the alternative he ranks higher, say $x^{*}$ and $y^{*}$. If he prefers $x^{*}$ to $y^{*}$, then he also prefers $X$ to $Y$. If he is indifferent between $x^{*}$ and $y^{*}$, then he is also indifferent between $X$ and $Y$.

${ }^{8}$ For each pair of chosen alternatives $(a, b)$, the choice of $a$ depends only on agents preferring $a$ over $b$.

${ }^{9} \mathrm{~A}$ double median function can be decomposed into two median rules, where for each preference profile each one selects one alternative.

${ }^{10}$ If the preferences of an agent change, then the other agents should all be made at least as well off or they should all be made at most as well off. See page 36 for a formal definition.
} 
comprised of the left-peaks function and the right-peaks function ${ }^{11}$ Miyagawa (2001).

The above two results are -loosely speaking- similar to the results of this paper; in Section 3.5.5 we discuss them and compare them with the results of this paper.

In line with most of the related literature, our main results also make use of either the property of peaks-onliness or a version of continuity adapted for our context (i.e., where an interval of alternatives is chosen). In addition, we also study the sub-cases where correspondences are either efficient, or anonymous, or both. Concisely, our results are the following. First, in the domain of single-peaked preferences, strategy-proofness and peaks-onliness characterize the class of generalized median correspondences (Theorem 3.1); and if anonymity is also required, then the sub-class of median correspondences is characterized (Theorem 3.2). Second, neither of these results holds in the domain of symmetric and single-peaked preferences, nor can in these results continuity substitute peaks-onliness (the counterexample on page 84). Third, in the domain of single-peaked preferences, strategyproofness, voter-sovereignty, and either peaks-onliness or continuity characterize the class of efficient generalized median correspondences (Theorem 3.3); and if anonymity is also required, then the sub-class of efficient median correspondences is characterized (Theorem 3.4). Finally, in the domain of symmetric and singlepeaked preferences, the classes of efficient generalized median correspondences and efficient median correspondences can be similarly characterized with one difference; due to peaks-onliness being inherent in the domain, continuity plays no role.

The chapter proceeds as follows. Section 3.2 explains the model and states a preliminary result. Section 3.3 includes the definitions of choice functions and correspondences, as well as the definition of the classes of such functions and correspondence we characterize. Section 3.4 contains the properties we are interested in and some further preliminary results. Finally, Section 3.5 contains all main results and characterizations, as well as a table summarizing our results.

\subsection{The model}

Consider a coalition (of agents) $N \equiv\{1, \ldots, n\}$, such that $n \geq 2$, and a set of alternatives $A \equiv[0,1] .{ }^{12}$ We denote generic agents by $i$ and $j$, and generic

\footnotetext{
${ }^{11}$ The left (right) peaks function chooses the two unique left-most (right-most) peaks.

${ }^{12}$ The set of alternatives is chosen without loss of generality. Essentially, our results hold for any closed interval in $\mathbb{R}$.
} 
alternatives by $x$ and $y$. Each $i$ is equipped with preferences $R_{i}$, defined over $A$, that are complete, transitive, and reflexive. As usual, $x R_{i} y$ is interpreted as " $x$ is at least as desirable as $y$ ", $x P_{i} y$ as " $x$ is preferred to $y$ ", and $x I_{i} y$ as " $x$ is indifferent to $y$ ". Moreover, for preferences $R_{i}$ there exists an alternative $p_{i} \in A$, called the peak of $i$, with the following property: if either $y<x \leq p_{i}$ or $y>x \geq p_{i}$, we have $x P_{i} y$. We call such preferences single-peaked and denote the domain of single-peaked preferences by $\mathcal{R}$. Furthermore, if for preferences $R_{i} \in \mathcal{R}$, $\left|x-p_{i}\right|=\left|y-p_{i}\right|$ implies $x I_{i} y$, then we say these preferences are symmetric and denote the domain of symmetric preferences by $\mathcal{S}$.

In the sequel, all notation and definitions refer to domain $\mathcal{R}$ but also apply to domain $\mathcal{S}$. Moreover, all results presented in this section hold in both domains.

Let $\mathcal{R}^{N}$ be the set of profiles $R \equiv\left(R_{i}\right)_{i \in N}$ such that for each $i \in N, R_{i} \in \mathcal{R}$. Given $R \in \mathcal{R}^{N}$ and $j \in N$, we also use $R$ and $\left(R_{-j}, R_{j}\right)$ interchangeably. For each $R \in \mathcal{R}^{N}$, we denote the vector of peaks of $R$ by $p \equiv\left(p_{i}\right)_{i \in N}$. Let the smallest peak in $R$ be $\underline{p} \equiv \min \left(\left\{p_{i}\right\}_{i \in N}\right)$ and the largest peak in $R$ be $\bar{p} \equiv \max \left(\left\{p_{i}\right\}_{i \in N}\right)$. Finally, let the convex hull of peaks in $R$ be $\operatorname{Conv}(p) \equiv[\underline{p}, \bar{p}]$.

Let the class of closed intervals in $A$ be denoted by $\mathcal{A}$. We denote generic sets in $\mathcal{A}$ by $X$ and $Y$. We denote the minimum of $X$ by $X \equiv \min (X)$ and the maximum of $X$ by $\bar{X} \equiv \max (X)$. For each $R_{i} \in \mathcal{R}$, we denote the best alternative( $s$ ) of $i$ in $X$ by $b_{R_{i}}(X) \equiv\left\{x \in X\right.$ : for each $\left.y \in X, x R_{i} y\right\}$ and the worst alternative(s) of $i$ in $X$ by $w_{R_{i}}(X) \equiv\left\{x \in X\right.$ : for each $\left.y \in X, y R_{i} x\right\}$. Note that single-peakedness of $R_{i}$ and non-emptiness of $X$ imply that the sets $b_{R_{i}}(X)$ and $w_{R_{i}}(X)$ contain one or two elements; specifically, if $b_{R_{i}}(X)$ (respectively, $w_{R_{i}}(X)$ ) contains two elements, agent $i$ is indifferent between them. It is with some abuse of notation that we treat sets $b_{R_{i}}(X)$ and $w_{R_{i}}(X)$ as if they are points and for each $x \in X$, we write $b_{R_{i}}(X) R_{i} x R_{i} w_{R_{i}}(X)$.

We extend all preferences $R_{i} \in \mathcal{R}$, defined over $A$, to preferences defined over $\mathcal{A}$ according to the "best-worst" extension of preferences characterized by Barberà et al. (1984). ${ }^{13}$ Specifically, when comparing two sets, an agent only considers his best and his worst point(s) in each of them. Therefore, an agent prefers $X$ to $Y$ if he prefers his best point(s) in $X$ to his best point(s) in $Y$ and his worst point(s) in $X$ to his worst point(s) in $Y$. The following definition also covers three more cases arising when an agent is indifferent between his best or worst point(s) in the

\footnotetext{
${ }^{13}$ Preferences $R_{i}^{\mathcal{A}}$ defined over $\mathcal{A}$ satisfy weak-dominance $\left(x P_{i}^{\mathcal{A}} y\right.$ implies $\left.\{x\} P_{i}^{\mathcal{A}}\{x, y\} P_{i}^{\mathcal{A}}\{y\}\right)$ and weak-independence (given triple $X, Y, Z \in \mathcal{A}$ such that $[X \cap Z]=[Y \cap Z]=\emptyset, X P_{i}^{\mathcal{A}} Y$ implies $\left.[X \cup Z] R_{i}^{\mathcal{A}}[Y \cup Z]\right)$ if and only if $i$ compares sets in $\mathcal{A}$ according to the "best-worst" extension of preferences. Examples illustrating the reasoning behind requiring these properties (in a slightly different model) are provided in Klaus and Protopapas (2016).
} 
two sets.

With some abuse of notation, we use the same symbols to denote preferences over alternatives and preferences over sets of alternatives.

Best-worst extension of preferences. For each $i \in N$ with preferences $R_{i} \in \mathcal{R}$, and each pair $X, Y \in \mathcal{A}$,

$$
\begin{aligned}
& X R_{i} Y \text { if and only if }\left\{\begin{array}{r}
b_{R_{i}}(X) R_{i} b_{R_{i}}(Y) \\
\text { and } \\
w_{R_{i}}(X) R_{i} w_{R_{i}}(Y) .
\end{array}\right. \\
& \text { and } \\
& X P_{i} Y \text { if and only if } X R_{i} Y \text { and }\left\{\begin{array}{l}
b_{R_{i}}(X) P_{i} b_{R_{i}}(Y) \\
\text { or } \\
w_{R_{i}}(X) P_{i} w_{R_{i}}(Y) .
\end{array}\right.
\end{aligned}
$$

This extension of preferences is transitive: for each triple $X, Y, Z \in \mathcal{A}$, if $X R_{i} Y$ and $Y R_{i} Z$, then $X R_{i} Z$. However, it is not complete: there exist $X, Y \in \mathcal{A}$ such that neither $X R_{i} Y$ nor $Y R_{i} X$. To be precise, we now introduce the following definition.

Comparability. Given preferences $R_{i} \in \mathcal{R}$, sets $X, Y \in \mathcal{A}$ are comparable if and only if $\left[b_{R_{i}}(X) P_{i} b_{R_{i}}(Y)\right.$ implies $\left.w_{R_{i}}(X) R_{i} w_{R_{i}}(Y)\right]$ and $\left[w_{R_{i}}(X) P_{i} w_{R_{i}}(Y)\right.$ implies $\left.b_{R_{i}}(X) R_{i} b_{R_{i}}(Y)\right]$.

Based on the best-worst extension of preferences, we now define (Pareto) efficient sets.

Efficient sets. Given profile $R \in \mathcal{R}^{N}$, set $X \in \mathcal{A}$ is efficient if and only if there is no set $Y \in \mathcal{A}$ such that for each $i \in N, Y R_{i} X$, and for at least one $j \in N$, $Y P_{j} X$; we denote the class containing all efficient sets at $R$ by $\mathrm{E}(R)$.

We now present a characterization of efficient sets in this setting that follows from Klaus and Protopapas (2016). Note that the original result is a little more complicated since it holds for all compact sets.

Proposition 3.1 (Klaus and Protopapas (2016)). At profile $R \in \mathcal{R}^{N}$, a closed interval is efficient if and only if it is a subset of the convex hull of peaks in $R$. 


\subsection{Choice functions and correspondences}

In the sequel, all notation and definitions refer to domain $\mathcal{R}$ but also apply to domain $\mathcal{S}$. Moreover, all results presented in this section hold in both domains.

Each $i \in N$, announces preferences $V_{i} \in \mathcal{R}$ with associated announced peak $v_{i} \in A$. Given (true) profile $R \in \mathcal{R}^{N}$, if $V_{i}=R_{i}$, we say that $i$ is sincere; otherwise, if $V_{i} \neq R_{i}$, we say that $i$ deviates. All terminology, notation, and results of Section 3.2, defined for preferences $R_{i} \in \mathcal{R}$, are carried over to announced preferences $V_{i} \in \mathcal{R}$ by replacing $R$ and $p$ by $V$ and $v$ respectively, and adding the term "announced" as necessary. For example, since in profile $R \in \mathcal{R}^{N}$ the smallest peak is denoted by $\underline{p} \equiv \min \left(\left\{p_{i}\right\}_{i \in N}\right)$, in announced profile $V_{N} \in \mathcal{R}^{N}$ the smallest announced peak is denoted by $\underline{v} \equiv \min \left(\left\{v_{i}\right\}_{i \in N}\right)$.

A (choice) correspondence $F$ assigns to each $V \in \mathcal{R}^{N}$ a set $F(V) \in \mathcal{A}$, i.e., $F: \mathcal{R}^{N} \rightarrow \mathcal{A}$. Given $V \in \mathcal{R}^{N}$, let the minimum of $F(V)$ be $F(V) \equiv \min \{F(V)\}$ and the maximum of $F(V)$ be $\bar{F}(V) \equiv \max \{F(V)\}$. We denote the family of correspondences by $\mathcal{F}$. Moreover, if a correspondence $F \in \mathcal{F}$ assigns to each $V \in \mathcal{R}^{N}$ an interval consisting of a single point we will refer to it as a function and use notation $f \in \mathcal{f}$, i.e., $f: \mathcal{R}^{N} \rightarrow A$.

Before defining in Sections 3.3.1 and 3.3.2 two classes of functions and correspondences that our results revolve around, the following definition is necessary: for each odd and positive integer $k$, and each vector $T \in \mathbb{R}^{k}$, label the coordinates of $T$ such that $t_{1} \leq \cdots \leq t_{k}$; we define the median (coordinate) of $T$ by $\operatorname{med}(T) \equiv t_{\frac{k+1}{2}}$

Finally, we (would like the reader to notice) that the classes of generalized median rules and correspondences, defined in Section 3.3.1, are as the name suggests, a generalization of the classes of median rules and correspondences, defined in Section 3.3.2. Loosely speaking, this generalization boils down to the agents influencing the chosen interval non-symmetrically. This is discussed in Remark 3.3 (Section 3.3.2). The reason behind this sequencing is simple: our results for the classes of generalized median rules and correspondences can be easily shown to hold for the subclasses of median rules and correspondences respectively.

\subsubsection{Generalized median rules and correspondences}

The first class of functions we consider was introduced under the name strategy-proof voting schemes and characterized by strategy-proofness ${ }^{14}$ and peaks-

\footnotetext{
${ }^{14}$ No agent gains by deviating.
} 
onliness $^{15}$ (Moulin, 1980, Proposition 3). It was later shown that peaks-onliness can be substituted with the "weaker" property of continuity ${ }^{16}$ (Ching, 1997, Theorem). In order to provide a useful intuition in understanding this class, we present an example inspired by the one provided in Arribillaga and Massó (2016, p. 564).

Example 3.1. Consider the two agent case, i.e., $N=\{1,2\}$ and choose a 4dimensional vector $\alpha=\left(\alpha_{\emptyset}, \alpha_{\{1\}}, \alpha_{\{2\}}, \alpha_{N}\right)$ such that $\alpha_{N} \leq \alpha_{\{1\}} \leq \alpha_{\{2\}} \leq \alpha_{\emptyset}$. Next, define the function $f^{\alpha} \in \mathcal{f}$ as follows. For each $V \in \mathcal{R}^{N}$, if $v_{1} \leq v_{2}$, choose $\tilde{\alpha}_{v}=\left(\alpha_{\emptyset}, \alpha_{\{1\}}, \alpha_{N}\right)$ and set $f^{\alpha}(V)=\operatorname{med}\left(\tilde{\alpha}_{v}, v\right)$, and if $v_{1}>v_{2}$, choose $\tilde{\alpha}_{v}=\left(\alpha_{\emptyset}, \alpha_{\{2\}}, \alpha_{N}\right)$ and set $f^{\alpha}(V)=\operatorname{med}\left(\tilde{\alpha}_{v}, v\right)$.

Notice that if $\alpha_{\{1\}} \neq \alpha_{\{2\}}$, then the agents have asymmetric power in influencing the chosen alternative. Before discussing further this asymmetry, in an effort to shed more light on the behavior of $f^{\alpha}$, we first provide a second definition of it.

$$
\text { For each } V \in \mathcal{R}^{N}, f^{\alpha}(V)= \begin{cases}\alpha_{N} & \text { if } v_{1}, v_{2} \leq \alpha_{N} \\ v_{2} & \text { if } v_{1} \leq \alpha_{N} \leq v_{2} \leq \alpha_{\{1\}} \\ \alpha_{\{1\}} & \text { if } v_{1} \leq \alpha_{N} \leq \alpha_{\{1\}} \leq v_{2} \\ \operatorname{med}\left(v_{1}, v_{2}, \alpha_{\{1\}}\right) & \text { if } \alpha_{N} \leq v_{1} \leq \alpha_{\{1\}} \\ v_{1} & \text { if } \alpha_{\{1\}} \leq v_{1} \leq \alpha_{\{2\}} \\ \operatorname{med}\left(v_{1}, v_{2}, \alpha_{\{2\}}\right) & \text { if } \alpha_{\{2\}} \leq v_{1} \leq \alpha_{\emptyset} \\ \alpha_{\{2\}} & \text { if } v_{2} \leq \alpha_{\{2\}} \leq \alpha_{\emptyset} \leq v_{1} \\ v_{2} & \text { if } \alpha_{\{2\}} \leq v_{2} \leq \alpha_{\emptyset} \leq v_{1}, \text { and } \\ \alpha_{\emptyset} & \text { if } \alpha_{\emptyset} \leq v_{1}, v_{2} .\end{cases}
$$

It is easy to see from this second definition that the range of $f^{\alpha}$ equals $\left[\alpha_{N}, \alpha_{\emptyset}\right]$. Hence, this function can be interpreted as one assigning to agents 1 and 2 the power to choose an alternative from the interval $\left[\alpha_{N}, \alpha_{\emptyset}\right]$. Furthermore, as already briefly discussed, this power is not symmetric among the agents but depends on the choice of $\alpha_{\{1\}}$ and $\alpha_{\{2\}}$. For instance in this example, since $\alpha_{\{1\}} \leq \alpha_{\{2\}}$, agent 1 has a greater power than agent 2 in influencing the chosen alternative. To see this, first consider agent 1. He can make sure that the chosen alternative is not larger than $\alpha_{\{1\}}$ and not smaller than $v_{1}$ (by announcing $v_{1} \leq \alpha_{\{1\}}$ ), or that it is not larger than $v_{1}$ and not smaller than $\alpha_{\{1\}}$ (by announcing $v_{1} \geq \alpha_{\{1\}}$ ). In

\footnotetext{
${ }^{15}$ The chosen alternative only depends on the vector of announced peaks.

${ }^{16}$ If the announced preferences change a "little", the chosen alternative does not change "a lot".
} 
addition, he is a dictator on the interval $\left[\alpha_{\{1\}}, \alpha_{\{2\}}\right]$.

Next, consider agent 2. He only has the power to influence the chosen alternative if agent 1 "allows" him to do so. That is, if $\alpha_{N} \leq v_{1} \leq \alpha_{\{1\}}$, then agent 2 can pinpoint the chosen alternative on the interval $\left[v_{1}, \alpha_{\{1\}}\right]$, and if $v_{1} \leq \alpha_{N} \leq \alpha_{\{1\}}$, then agent 2 can pinpoint the chosen alternative on the interval $\left[\alpha_{N}, \alpha_{\{1\}}\right]$. Similarly, if $\alpha_{\{2\}} \leq v_{1} \leq \alpha_{\emptyset}$, then agent 2 can pinpoint the chosen alternative on the interval $\left[\alpha_{\{2\}}, v_{1}\right]$, and if $\alpha_{\{2\}} \leq \alpha_{\emptyset} \leq v_{1}$, then agent 2 can pinpoint the chosen alternative on the interval $\left[\alpha_{\{2\}}, \alpha_{\emptyset}\right]$.

The general $n$-agent case works as follows. First, take a vector $\alpha$ in $A^{2^{n}}$, i.e., the dimension of $\alpha$ equals the number of all sub-coalitions in $N$ (including the empty set). Specifically, let $\alpha \equiv\left(\alpha_{M}\right)_{M \subseteq N}$, such that for each $L \subseteq M$, $\alpha_{L} \geq \alpha_{M}$. Next, for an announced profile $V$ with associated vector of announced peaks $v$, label the agents such that $v_{\overline{1}} \leq \cdots \leq v_{\bar{n}}{ }^{17}$ Finally, construct vector $\tilde{\alpha}_{v}$ in $A^{n+1}$ such that $\tilde{\alpha}_{v}=\left(\alpha_{\emptyset}, \alpha_{\{\overline{1}\}}, \alpha_{\{\overline{1}, \overline{2}\}}, \ldots, \alpha_{N}\right)$ and notice that by construction, $\alpha_{N} \leq \cdots \leq \alpha_{\{\overline{1}, \overline{2}\}} \leq \alpha_{\{\overline{1}\}} \leq \alpha_{\emptyset}$. The generalized median rule associated with vector $\alpha$ chooses alternative $\operatorname{med}\left(v, \tilde{\alpha}_{v}\right)$.

Generalized median rules. Let vector $\alpha \in A^{2^{n}}$ be such that $\alpha \equiv\left(\alpha_{M}\right)_{M \subseteq N}$, where for each pair $L, M \subseteq N$ with $L \subsetneq M, \alpha_{L} \geq \alpha_{M}$. Also, for each $V \in \mathcal{R}^{N}$, let bijection $\pi: N \rightarrow N$ be such that $v_{\pi(1)} \leq \cdots \leq v_{\pi(n)}$ and construct vector $\tilde{\alpha}_{v}=$ $\left(\alpha_{\emptyset}, \alpha_{\{\pi(1)\}}, \alpha_{\{\pi(1), \pi(2)\}}, \ldots, \alpha_{N}\right)$. We denote the generalized median rule associated with vector $\alpha$ by $f_{G}^{\alpha}$, where for each $V \in \mathcal{R}^{N}, f_{G}^{\alpha}(V) \equiv \operatorname{med}\left(v, \tilde{\alpha}_{v}\right)$. Finally, we denote the class of generalized median rules by $\mathcal{f}_{G} .{ }^{18}$

Clearly, if all agents announce different peaks, a unique ordering of them by their announced peak exists. Moreover, for the case where some agents announce the same peak and hence such a unique ordering does not exist, the chosen alternative does not depend on the particular ordering chosen; as shown in Ching (1997, Remark 1).

The first class of correspondences we characterize in Section 3.5 extends the spirit of generalized median rules to correspondences. Specifically, take two vectors $\alpha \leq \beta$, each of dimension $2^{n}$, such that $\alpha \equiv\left(\alpha_{M}\right)_{M \subseteq N}$ and $\beta \equiv\left(\beta_{M}\right)_{M \subseteq N}$. Next, for an announced profile $V$ with associated vector of announced peaks $v$, label the

\footnotetext{
${ }^{17}$ Whenever two agents announce the same peak, no unique way to label the agents exists. However, the specific choice of labels does not affect the chosen alternative (Ching, 1997, Remark 1).

${ }^{18}$ It should be noted that in the literature a generalized median rule $f_{G}^{\alpha} \in \mathcal{F}_{G}$ is often described as follows: For each $V \in \mathcal{R}^{N}, f_{G}^{\alpha}(V)=\min _{M \subseteq N} \max _{i \in M}\left\{v_{i}, \alpha_{M}\right\}$.
} 
agents such that $v_{1} \leq \cdots \leq v_{n}$ and construct vectors $\tilde{\alpha}_{v}$ and $\tilde{\beta}_{v}$, each of dimension $n+1$, such that $\tilde{\alpha}_{v}=\left(\alpha_{\emptyset}, \alpha_{\{1\}}, \alpha_{\{1,2\}}, \ldots, \alpha_{N}\right)$ and $\tilde{\beta}_{v}=\left(\beta_{\emptyset}, \beta_{\{1\}}, \beta_{\{1,2\}}, \ldots, \beta_{N}\right)$. The generalized median correspondence associated with vectors $\alpha$ and $\beta$ chooses the interval where the minimum alternative is $\operatorname{med}\left(v, \tilde{\alpha}_{v}\right)$ and the maximum alternative is $\operatorname{med}\left(v, \tilde{\beta}_{v}\right)$.

Generalized median correspondences. Let vectors $\alpha, \beta \in A^{2^{n}}$ be such that $\alpha \equiv\left(\alpha_{M}\right)_{M \subseteq N}$ and $\beta \equiv\left(\beta_{M}\right)_{M \subseteq N}$, with $\alpha \leq \beta$, and for each pair $L, M \subseteq N$, with $L \subsetneq M, \alpha_{L} \geq \alpha_{M}$ and $\beta_{L} \geq \beta_{M}$. Also, for each $V \in \mathcal{R}^{N}$, let bijection $\pi: N \rightarrow N$ such that $v_{\pi(1)} \leq \cdots \leq v_{\pi(n)}$ and construct vectors $\tilde{\alpha}_{v}=$ $\left(\alpha_{\emptyset}, \alpha_{\{\pi(1)\}}, \alpha_{\{\pi(1), \pi(2)\}}, \ldots, \alpha_{N}\right)$ and $\tilde{\beta}_{v}=\left(\beta_{\emptyset}, \beta_{\{\pi(1)\}}, \beta_{\{\pi(1), \pi(2)\}}, \ldots, \beta_{N}\right)$. We denote the generalized median correspondence associated with vectors $\alpha$ and $\beta$ by $F_{G}^{\alpha, \beta}$, where for each $V \in \mathcal{R}^{N}, F_{G}^{\alpha, \beta}(V) \equiv\left[\operatorname{med}\left(v, \tilde{\alpha}_{v}\right), \operatorname{med}\left(v, \tilde{\beta}_{v}\right)\right]$. Finally, we denote the class of generalized median correspondences by $\mathcal{F}_{G}$.

Remark 3.1. By definition of $\mathcal{F}_{G}$ and $f_{G}$, for each profile $V, F_{G}^{\alpha, \beta}(V) \equiv$ $\left[\operatorname{med}\left(v, \tilde{\alpha}_{v}\right), \operatorname{med}\left(v, \tilde{\beta}_{v}\right)\right]=\left[f_{G}^{\alpha}(V), f_{G}^{\beta}(V)\right]$. Therefore, a generalized median correspondence $F_{G}^{\alpha, \beta}$ can be decomposed into two generalized median rules $f_{G}^{\alpha}$ and $f_{G}^{\beta}$

The next result considers single-valued generalized median correspondences.

Lemma 3.1. A generalized median correspondence $F_{G}^{\alpha, \beta}$ is single-valued if and only if $\alpha=\beta$. Moreover, in this case $F_{G}^{\alpha, \beta}$ is essentially a generalized median rule. $^{19}$

Proof. Let $F_{G}^{\alpha, \beta} \in \mathcal{F}_{G}$ and $f_{G}^{\alpha}, f_{G}^{\beta} \in \mathcal{f}_{G}$. Let $V \in \mathcal{R}^{N}$. By Remark 3.1, $F_{G}^{\alpha, \beta}(V)=$ $\left[f_{G}^{\alpha}(V), f_{G}^{\beta}(V)\right]$. If $\alpha=\beta$, then $F_{G}^{\alpha, \beta}(V)=\left\{f_{G}^{\alpha}(V)\right\}$. Hence, $F_{G}^{\alpha, \beta}$ is single-valued.

If $F_{G}^{\alpha, \beta}$ is single-valued, then $F_{G}^{\alpha, \beta}(V)=\left[f_{G}^{\alpha}(V), f_{G}^{\beta}(V)\right]$ implies $f_{G}^{\alpha}(V)=f_{G}^{\beta}(V)$. Assuming $\alpha \neq \beta$ results in a contradiction as follows. Since $\alpha \neq \beta$, there exists a coalition $M \subseteq N$ such that $\alpha_{M} \neq \beta_{M}$. Let $|M|=m$ and specify $V$ such that for each agent $i \in M, v_{i}=0$, and for each agent $j \in N \backslash M, v_{j}=1$. Hence, at profile $V$, the $m$ th coordinate of vectors $\tilde{\alpha}_{v}$ and $\tilde{\beta}_{v}$ will be $\alpha_{M}$ and $\beta_{M}$ respectively. Moreover, by definition of $\mathcal{F}_{G}, \alpha_{N} \leq \alpha_{\{1, \ldots, n-1\}} \leq \cdots \leq \alpha_{\{1\}} \leq \alpha_{\emptyset}$ and $\beta_{N} \leq$ $\beta_{\{1, \ldots, n-1\}} \leq \cdots \leq \beta_{\{1\}} \leq \beta_{\emptyset}$. Thus, there are at least $n+1-m$ coordinates of $\tilde{\alpha}_{v}$ not larger than $\alpha_{M}$ (i.e., coordinates $\alpha_{M}, \ldots, \alpha_{N}$ ) and at least $m+1$ coordinates of $\tilde{\alpha}_{v}$ not smaller than $\alpha_{M}$ (i.e., coordinates $\left.\alpha_{\emptyset}, \ldots, \alpha_{M}\right)$. Similarly, there are at least

\footnotetext{
${ }^{19}$ To be precise, a single-valued generalized median correspondence assigns singleton sets of alternatives while the corresponding generalized median rule assigns the alternatives contained in these sets.
} 
$n+1-m$ coordinates of $\tilde{\beta}_{v}$ not larger than $\beta_{M}$ and at least $m+1$ coordinates of $\tilde{\beta}_{v}$ not smaller than $\beta_{M}$. Hence, $F_{G}^{\alpha, \beta}=\left[\operatorname{med}\left(v, \tilde{\alpha}_{v}\right), \operatorname{med}\left(v, \tilde{\beta}_{v}\right)\right]=\left[\alpha_{M}, \beta_{M}\right]$ contradicting that $F_{G}^{\alpha, \beta}$ is single-valued. Therefore, $F_{G}^{\alpha, \beta}(V)$ being single-valued implies $\alpha=\beta$.

Our results in Section 3.5 will also concern efficient generalized median correspondences. Formally, given $F_{G}^{\alpha, \beta} \in \mathcal{F}_{G}$, if for each $V \in \mathcal{R}^{N}, F_{G}^{\alpha, \beta} \in \mathrm{E}(V)$, we say that $F_{G}^{\alpha, \beta}$ is an efficient generalized median correspondence and denote the class of efficient generalized median correspondences by $\mathcal{F}_{E G}$. The next result concerns this class of correspondences.

Lemma 3.2. A generalized median correspondence $F_{G}^{\alpha, \beta}$ is an efficient generalized median correspondence if and only if vectors $\alpha, \beta$ are such that $\alpha_{N}=\beta_{N}=0$ and $\alpha_{\emptyset}=\beta_{\emptyset}=1$.

Proof. Let $F_{G}^{\alpha, \beta} \in \mathcal{F}_{G}$. Assuming that $F_{G}^{\alpha, \beta} \in \mathcal{F}_{E G}$ such that $\alpha, \beta$ are not as described above, results in a contradiction as follows.

If $\alpha_{N} \neq 0$ or $\beta_{N} \neq 0$, choose $V \in \mathcal{R}^{N}$ such that $v=(0, \ldots, 0)$. By Proposition 3.1, $\mathrm{E}(V)=\{0\}$ and by the definition of $\mathcal{F}_{G}, F_{G}^{\alpha, \beta}(V)=\left[\alpha_{N}, \beta_{N}\right]$. Hence, $F_{G}^{\alpha, \beta}(V) \notin \mathrm{E}(V)$. Similarly, if $\alpha_{\emptyset} \neq 1$ or $\beta_{\emptyset} \neq 1$, choose $V \in \mathcal{R}^{N}$ such that $v=(1, \ldots, 1)$. Again, by Proposition $3.1, \mathrm{E}(V)=\{1\}$ and by the definition of $\mathcal{F}_{G}, F_{G}^{\alpha, \beta}(V)=\left[\alpha_{\emptyset}, \beta_{\emptyset}\right]$. Hence, $F_{G}^{\alpha, \beta}(V) \notin \mathrm{E}(V)$.

Finally, if $\alpha_{N}=\beta_{N}=0$ and $\alpha_{\emptyset}=\beta_{\emptyset}=1$, then for each $V \in \mathcal{R}^{N}, \operatorname{med}\left(v, \tilde{\alpha}_{v}\right) \in$ $\operatorname{Conv}(v)$ and $\operatorname{med}\left(v, \tilde{\beta}_{v}\right) \in \operatorname{Conv}(v)$. Hence, by the definition of $\mathcal{F}_{G}, F_{G}^{\alpha, \beta}(V) \subseteq$ $\operatorname{Conv}(v)$, and thus by Proposition 3.1, $F_{G}^{\alpha, \beta}(V) \in \mathrm{E}(V)$.

\subsubsection{Median rules and correspondences}

The second class of functions we consider was introduced under the name strategyproof and anonymous voting schemes and characterized by strategy-proofness, peaks-onliness, and anonymity ${ }^{20}$ (Moulin, 1980, Proposition 2). As discussed in Remark 3.3, this class of functions is a subclass of generalized median rules since now all agents possess the same power in influencing the chosen alternative. For the 2-agent case, it suffices to set $\alpha_{\{1\}}=\alpha_{\{2\}}$ in Example 3.1 (page 74).

The general $n$-agent case works as follows. Take a vector $a$ in $A^{n+1}$. For an announced profile $V$ with associated vector of announced peaks $v$, the median rule associated with $a$ chooses alternative $\operatorname{med}(v, a)$.

\footnotetext{
${ }^{20}$ The names of the agents do not affect the chosen alternative.
} 
Median rules. Let vector $a \in A^{n+1}$ be such that $a \equiv\left(a_{1}, \ldots, a_{n+1}\right)$, where $a_{1} \leq$ $\cdots \leq a_{n+1}$. We denote the median rule associated with vector a by $f_{M}^{a}$, where for each $V \in \mathcal{R}^{N}, f_{M}^{a}(V) \equiv \operatorname{med}(v, a)$. Finally, we denote the class of median rules by $f_{M}$.

The second class of correspondences we characterize in Section 3.5 extends the spirit of median rules to correspondences. Specifically, take two vectors $a \leq b$, each of dimension $n+1$. For an announced profile $V$ with associated vector of announced peaks $v$, the median correspondence associated with $a$ and $b$ chooses the interval where the minimum alternative is $\operatorname{med}(v, a)$ and the maximum alternative is $\operatorname{med}(v, b)$.

Median correspondences. Let vectors $a, b \in A^{n+1}$ be such that $a \equiv$ $\left(a_{1}, \ldots, a_{n+1}\right)$ and $b \equiv\left(b_{1}, \ldots, b_{n+1}\right)$, with $a \leq b, a_{1} \leq \cdots \leq a_{n+1}$, and $b_{1} \leq \cdots \leq b_{n+1}$. We denote the median correspondence associated with vectors $a$ and $b$ by $f_{M}^{a, b}$, where for each $V \in \mathcal{R}^{N}, F_{M}^{a, b}(V) \equiv[\operatorname{med}(v, a), \operatorname{med}(v, b)]$. Finally, we denote the class of median correspondences by $\mathcal{F}_{M}$.

Remark 3.2. By definition of $\mathcal{F}_{M}$ and $\mathcal{f}_{M}$, for each profile $V, F_{M}^{a, b}(V) \equiv$ $[\operatorname{med}(v, a), \operatorname{med}(v, b)]=\left[f_{M}^{a}(V), f_{M}^{b}(V)\right]$. Therefore, a median correspondence $F_{M}^{a, b}$ can be decomposed into two median rules $f_{M}^{a}$ and $f_{M}^{b}$.

Remark 3.3. The class of median rules (correspondences) is a subclass of the class of generalized median rules (correspondences). That is, given $F_{M}^{a, b} \in \mathcal{F}_{M}$, let $F_{G}^{\alpha, \beta} \in \mathcal{F}_{G}$ by choosing vectors $\alpha, \beta \in A^{2^{n}}$ such that the weight of each coalition only depends on its cardinality. Specifically, for each $M \subseteq N$, choose $\alpha_{M}=$ $a_{n+1-|M|}$ (i.e., choose $\alpha_{\emptyset}=a_{n+1}$, for each $i \in N$, choose $\alpha_{\{i\}}=a_{n}$, for each $i, j \in N$ with $i \neq j$, choose $\alpha_{\{i, j\}}=a_{n-1}$, and so on) and $\beta_{M}=b_{n+1-|M|}$. It follows that for each $V \in \mathcal{R}^{N}, \tilde{\alpha}_{v}=a$ and $\tilde{\beta}_{v}=b$, implying $F_{M}^{a, b}(V)=F_{G}^{\alpha, \beta}(V)$.

The next result considers single-valued median correspondences.

Lemma 3.3. A median correspondence $F_{M}^{a, b}$ is single-valued if and only if $a=b$. Moreover, in this case $F_{M}^{a, b}$ is essentially a median rule. ${ }^{21}$

Lemma 3.3 follows from Lemma 3.1 and Remark 3.3.

Our results in Section 3.5 also concern efficient median correspondences. Formally, given $F_{M}^{a, b} \in \mathcal{F}_{M}$, if for each $V \in \mathcal{R}^{N}, F_{M}^{a, b} \in \mathrm{E}(V)$, we say that $F_{M}^{a, b}$ is an efficient median correspondence and denote the class of efficient median correspondences by $\mathcal{F}_{E M}$. The next result concerns this class of correspondences.

\footnotetext{
${ }^{21}$ To be precise, a single-valued median correspondence assigns singleton sets of alternatives while the corresponding median rule assigns the alternatives contained in these sets.
} 
Lemma 3.4. A median correspondence $F_{M}^{a, b}$ is an efficient median correspondence if and only if vectors $a, b$ are such that $a_{1}=b_{1}=0$ and $a_{n+1}=b_{n+1}=1$.

Lemma 3.4 follows from Lemma 3.2 and Remark 3.3.

Remark 3.4. As discussed in Section 3.5, both classes of generalized median correspondences and median correspondences are strategy-proof ${ }^{22}$, similar to the results on functions by Moulin (1980). However, it should be noted that in contrast to Moulin's results these classes of correspondences are not group strategy-proof. ${ }^{23}$ The following example illustrates this.

Example 3.2. Let $N=\{1,2,3\}$ and $F^{a, b} \in \mathcal{F}_{E M}$ such that $a=\{0,0,0,1\}$ and $b=\{0,0,1,1\}$. In addition, let $V, V^{\prime} \in \mathcal{S}$ be such that $v_{1}=v_{1}^{\prime}=0, v_{2}=0.5$, $v_{2}^{\prime}=0.6$, and $v_{3}=v_{3}^{\prime}=1$. Then, $F(V)=[0,0.5]$ and $F\left(V^{\prime}\right)=[0,0.6]$. Therefore, assuming $V$ are the true preferences of the agents implies that although agent 2 is indifferent to deviating, by announcing $V_{2}^{\prime}$, agent 3 gains by this deviation.

\subsection{Properties of correspondences}

In the sequel, all properties are defined for correspondences in domain $\mathcal{R}$ but also apply to correspondences in domain $\mathcal{S}$. Moreover, all results presented in this section hold in both domains.

The first two properties we consider are related; the first is our efficiency notion for correspondences while the second, being weaker than the first, requires no alternative in $A$ to be a priori excluded from being selected.

Efficiency. For each $V \in \mathcal{R}^{N}, F(V) \in \mathrm{E}(V)$.

Voter-sovereignty. For each $x \in A$, there exists $V \in \mathcal{R}^{N}$ such that $F(V)=\{x\}$.

The next property, which is central in our results, requires no agent to gain by deviating. Moreover, it implies comparability between the chosen sets before and after an agent's deviation.

Strategy-proofness. For each $i \in N$, each $R_{i} \in \mathcal{R}$, and each $V \in \mathcal{R}^{N}$, $F\left(V_{-i}, R_{i}\right) R_{i} F\left(V_{-i}, V_{i}\right)$.

The next property requires the chosen set to depend only on the vector of announced peaks.

\footnotetext{
${ }^{22}$ No agent gains by deviating. See Section 3.5.1 for a formal proof.

${ }^{23}$ No group of agents can deviate such that some members of the group gain and no member of the group loses.
} 
Peaks-onliness. For each pair $V, V^{\prime} \in \mathcal{R}^{N}$ such that $v=v^{\prime}, F(V)=F\left(V^{\prime}\right)$.

Loosely speaking, the next property requires when the announced preferences of an agent change "a little", the minimum and maximum alternatives chosen to not change "a lot". Before describing it formally, we must first define the three following notions. First, the "indifference relation", which -loosely speaking- given preferences $V_{i} \in \mathcal{R}$, maps each alternative $x$ to an alternative $y$, that $i$ finds indifferent to $x$, according to $V_{i}$. Formally, for each $V_{i} \in \mathcal{R}$, the indifference relation $r_{V_{i}}:[0,1] \rightarrow[0,1]$ is defined as follows. For each $x \in\left[0, v_{i}\right], r_{V_{i}}(x)=y$ if $y \in\left[v_{i}, 1\right]$ exists such that $y I_{i} x$, or $r_{V_{i}}(x)=1$ otherwise; while for each $x \in$ $\left[v_{i}, 1\right], r_{V_{i}}(x)=y$ if $y \in\left[0, v_{i}\right]$ exists such that $y I_{i} x$, or $r_{V_{i}}(x)=0$ otherwise. Second, the distance between a pair $V_{i}, V_{i}^{\prime} \in \mathcal{R}$, which is measured using the indifference relation. Formally, it is defined to be $\mathrm{d}\left(V_{i}, V_{i}^{\prime}\right) \equiv \max _{x \in[0,1]} \mid r_{V_{i}}(x)-$ $r_{V_{i}^{\prime}}(x) \mid$. Finally, the notion of convergence. Specifically, for $k \in \mathbb{N}^{+}$, a sequence $\left\{V_{i}^{k}\right\}$ in $\mathcal{R}$ converges to $V_{i}$, if $k \rightarrow \infty$ implies the distance $\mathrm{d}\left(V_{i}, V_{i}^{k}\right) \rightarrow 0$. We denote this convergence by $V_{i}^{k} \rightarrow V_{i}$.

Min/max continuity. For each $V \in \mathcal{R}^{N}$, each $i \in N$, and each $\left\{V_{i}^{k}\right\}$ in $\mathcal{R}$,

$$
\text { if } V_{i}^{k} \rightarrow V_{i}, \text { then }\left\{\begin{array}{l}
\underline{F}\left(V_{-i}, V_{i}^{k}\right) \rightarrow \underline{F}(V), \text { and } \\
\bar{F}\left(V_{-i}, V_{i}^{k}\right) \rightarrow \bar{F}(V) .
\end{array}\right.
$$

Notice that $\min / \max$ continuity for functions is equivalent to the regular continuity property for functions (with respect to the preference profile). Moreover, in Appendix 3.A we show the equivalence of this property with the standard continuity properties for correspondences, upper-hemi continuity and lower-hemi continuity.

The next property requires that the agents' identities do not matter.

Anonymity. For each bijection $\sigma: N \rightarrow N$ and each pair $V, V^{\prime} \in \mathcal{R}^{N}$ such that for each $i \in N, V_{i}=V_{\sigma(i)}^{\prime}, F(V)=F\left(V^{\prime}\right)$.

The last property we consider depends only on the announced peaks of the agents. Loosely speaking, following a change in an agent's announced preferences, if before and after this change both announced peaks lie on the same side of the minimum (maximum) chosen alternative, then the minimum (maximum) chosen alternative does not change.

Uncompromisingness. For each $i \in N$ and each pair $V, V^{\prime} \in \mathcal{R}^{N}$ such that 
$V_{-i}^{\prime}=V_{-i}$

$$
\begin{aligned}
& \text { if }\left\{\begin{array}{l}
v_{i}<\underline{F}(V) \text { and } v_{i}^{\prime} \leq \underline{F}(V) \text { or } \\
v_{i}>\underline{F}(V) \text { and } v_{i}^{\prime} \geq \underline{F}(V),
\end{array} \text { then } \underline{F}(V)=\underline{F}\left(V^{\prime}\right)\right. \text {, and } \\
& \text { if }\left\{\begin{array}{l}
v_{i}<\bar{F}(V) \text { and } v_{i}^{\prime} \leq \bar{F}(V) \text { or } \\
v_{i}>\bar{F}(V) \text { and } v_{i}^{\prime} \geq \bar{F}(V),
\end{array} \text { then } \bar{F}(V)=\bar{F}\left(V^{\prime}\right)\right. \text {. }
\end{aligned}
$$

When a correspondence does not satisfy uncompromisingness, we say that it is compromised.

\subsection{Results}

We begin by presenting in Section 3.5.1 results concerning interrelations between the properties presented in Section 3.4. Said results are then used in our characterization results presented in Sections 3.5.2 and 3.5.3. Loosely speaking, in Section 3.5.2, we extend the characterizations of Moulin (1980, Propositions 2 and 3$)^{24}$ to correspondences in domain $\mathcal{R}$; while in Section 3.5.3, we show which of these characterizations can be extended in domain $\mathcal{S}$. Next, in section 3.5.4 we show that the properties in all our characterization results are independent; and finally, in section 3.5.5 we compare these results to those of Miyagawa (2001) and Heo (2013).

\subsubsection{Interrelations between properties}

Our first result in this section holds in domain $\mathcal{S}$. It shows that if strategyproofness is satisfied, then efficiency and voter-sovereignty are equivalent.

Proposition 3.2. For strategy-proof correspondences efficiency and votersovereignty are equivalent in domain $\mathcal{S}$.

We prove Proposition 3.2 in Appendix 3.B. In addition, a similar equivalence result holds in domain $\mathcal{R}$, albeit slightly weaker since peaks-onliness or $\mathrm{min} / \mathrm{max}$ continuity is also required. By Proposition 3.2, this result trivially holds in domain $\mathcal{S}$ as well

\footnotetext{
${ }^{24}$ Proposition 2: A function satisfies strategy-proofness, peaks-onliness, and anonymity in $\mathcal{R}$ if and only if it is a median rule. Proposition 3: A function satisfies strategy-proofness and peaks-onliness in $\mathcal{R}$ if and only if it is a generalized median rule.
} 
Proposition 3.3. The following two statements for strategy-proof correspondences hold.

(i) If peaks-onliness is satisfied, then efficiency and voter-sovereignty are equivalent.

(ii) If $\min / \max$ continuity is satisfied, then efficiency and voter-sovereignty are equivalent.

We prove Proposition 3.3 in Appendix 3.B. Moreover, the next result holds only in domain $\mathcal{R}{ }^{25}$ It shows that strategy-proofness and peaks-onliness are equivalent with uncompromisingness.

Proposition 3.4. The following two statements for correspondences are equivalent in domain $\mathcal{R}$.

(i) Strategy-proofness and peaks-onliness are satisfied.

(ii) Uncompromisingness is satisfied.

We prove Proposition 3.4 in Appendix 3.C. Moreover, the next result is in the spirit of Proposition 3.4 and holds in domain $\mathcal{S}$.

Proposition 3.5. The following two statements for correspondences are equivalent in domain $\mathcal{S}$.

(i) Strategy-proofness and voter-sovereignty are satisfied.

(ii) Uncompromisingness and voter-sovereignty are satisfied.

We prove Proposition 3.5 in Appendix 3.C. Moreover, our final result also concerns uncompromisingness. It holds in both domains $\mathcal{R}$ and $\mathcal{S}$.

Proposition 3.6. Each correspondence satisfying strategy-proofness, $\min / \max$ continuity, and voter-sovereignty also satisfies uncompromisingness.

We prove Proposition 3.6 in Appendix 3.C.

\subsubsection{Results in the single-peaked domain $\mathcal{R}$}

We now present our characterization results for (generalized) median correspondences, as well as a counter-example justifying the absence of such results in some cases. All results hold in domain $\mathcal{R}$. The extension of these results in domain $\mathcal{S}$ is discussed in Section 3.5.3.

Our first result concerns the class of generalized median correspondences.

\footnotetext{
${ }^{25}$ An example of Proposition 3.4 not holding in domain $\mathcal{S}$ is illustrated by the counter-example on page 84 .
} 
Theorem 3.1. The following three statements for a correspondence $F \in \mathcal{F}$ are equivalent.

(i) F satisfies strategy-proofness and peaks-onliness.

(ii) F satisfies uncompromisingness.

(iii) $F$ is a generalized median correspondence.

The equivalence of statements (i) and (ii) follows from Propostion 3.4. We prove the equivalence of statements (ii) and (iii) in Appendix 3.D; note that this part of the proof also holds in domain $\mathcal{S}$.

Our second result concerns the class of median correspondences.

Theorem 3.2. The following three statements for a correspondence $F \in \mathcal{F}$ are equivalent.

(i) F satisfies strategy-proofness, peaks-onliness, and anonymity.

(ii) F satisfies uncompromisingness and anonymity.

(iii) $F$ is a median correspondence.

Proof. The equivalence of statements (i) and (ii) follows from Propostion 3.4. We proceed by showing the equivalence of statements (ii) and (iii) in two steps; note that this part of the proof also holds in domain $\mathcal{S}$.

Step 1 - (statement (ii) implies statement (iii)): Let $F \in \mathcal{F}$ satisfy uncompromisingness and anonymity, and $V \in \mathcal{R}$. By Theorem 3.1, there exists $F_{G}^{\alpha, \beta} \in \mathcal{F}_{G}$ such that $F(V)=F_{G}^{\alpha, \beta}(V)$. Moreover, anonymity implies for $\alpha, \beta \in$ $A^{2^{n}}$ that for each pair $L, M \subseteq N$, if $|L|=|M|$, then $\alpha_{L}=\alpha_{M}$ and $\beta_{L}=\beta_{M}$. Thus, for each $M \subseteq N$, let $a_{n+1-|M|}=\alpha_{M}$ and $b_{n+1-|M|}=\beta_{M}$, to effectively construct vectors $a, b \in A^{n+1}$. Next, let $F_{M}^{a, b} \in \mathcal{F}_{M}$ and notice that for each $V \in \mathcal{R}^{N}$, $F_{G}^{\alpha, \beta}(V)=\left[\operatorname{med}\left(v, \tilde{\alpha}_{v}\right), \operatorname{med}\left(v, \tilde{\beta}_{v}\right)\right]=[\operatorname{med}(v, a), \operatorname{med}(v, b)]=F_{M}^{a, b}(V)$.

Step 2 - (statement (iii) implies statement (ii)): Let $F_{M}^{a, b} \in \mathcal{F}_{M}$. In addition, let $F_{G}^{\alpha, \beta} \in \mathcal{F}_{G}$ by choosing vectors $\alpha, \beta \in A^{2^{n}}$ such that the weight of each coalition only depends on its cardinality; specifically, for each $M \subseteq N, \alpha_{M}=a_{n+1-|M|}$ and $\beta_{M}=b_{n+1-|M|}$. Hence, for each $V \in \mathcal{R}^{N}$, $F_{G}^{\alpha, \beta}(V)=\left[\operatorname{med}\left(v, \tilde{\alpha}_{v}\right), \operatorname{med}\left(v, \tilde{\beta}_{v}\right)\right]=[\operatorname{med}(v, a), \operatorname{med}(v, b)]=F_{M}^{a, b}(V)$. Therefore, by Theorem 3.1, $F_{M}^{a, b}$ satisfies uncompromisingness and by the definition of $\mathcal{F}_{M}, F_{M}^{a, b}$ satisfies anonymity.

Next, we show that in Theorems 3.1 and 3.2 peaks-onliness cannot be substituted with $\mathrm{min} / \max$ continuity. We illustrate this in the counter-example that follows by exhibiting a correspondence satisfying strategy-proofness, min/max continuity, and anonymity and violating voter-sovereignty -and more importantly- 
uncompromisingness; which, as shown in the aforementioned theorems, is satisfied by both classes of generalized median correspondences and median correspondences. Moreover, as explained in Section 3.5.3, this example also illustrates that the aforementioned theorems cannot be extended in domain $\mathcal{S}$.

Counter-example. Let $|N| \geq 1$ and define $r_{V}^{*} \equiv \max \left\{r_{V_{i}}(0)\right\}_{i \in N}$, that is, at announced profile $V$, among the indifferent announced alternatives to 0 of each agent $i \in N, r_{V}^{*}$ is the largest one. Next, define $F^{*} \in \mathcal{F}$ as follows. For each $V \in \mathcal{R}^{N}, F^{*}(V)=\left[0, r_{V}^{*}\right]$. By definition, it follows that $F^{*}$ satisfies $\min / \max$ continuity and anonymity, and that it violates voter-sovereignty. We proceed in 2 steps

Step 1: We show $F^{*}$ satisfies strategy-proofness. Let $V \in \mathcal{R}^{N}\left(V \in \mathcal{S}^{N}\right)$ be such that $i \in N$ is sincere, i.e., $V_{i}=R_{i}$. Also, let $V_{i}^{\prime} \in \mathcal{R}\left(V_{i}^{\prime} \in \mathcal{S}\right)$ be such that $V_{i}^{\prime} \neq V_{i}$. There are two cases.

Case 1. Let $r_{V_{i}}(0)=r_{V}^{*}$. By single-peakedness, $b_{R_{i}}\left(F^{*}(V)\right)=\left\{p_{i}\right\}$, implying $i$ 's best point does not improve by deviating at $V$, and $0 \in w_{R_{i}}\left(F^{*}(V)\right)$. By the definition of $F^{*}, 0 \in F^{*}\left(V_{-i}, V_{i}^{\prime}\right)$, hence $i$ 's worst point(s) does not improve by deviating at $V$. Therefore, $F^{*}(V) R_{i} F^{*}\left(V_{-i}, V_{i}^{\prime}\right)$.

Case 2. Let $r_{V_{i}}(0)<r_{V}^{*}$. By single-peakedness, $b_{R_{i}}\left(F^{*}(V)\right)=\left\{p_{i}\right\}$, implying $i$ 's best point does not improve by deviating at $V$, and $w_{V_{i}}\left(F^{*}(V)\right)=\left\{r_{V}^{*}\right\}$. By the definition of $F^{*}, r_{V}^{*} \in F^{*}\left(V_{-i}, V_{i}^{\prime}\right)$, hence $i$ 's worst point does not improve by deviating at $V$. Therefore, $F^{*}(V) R_{i} F^{*}\left(V_{-i}, V_{i}^{\prime}\right)$.

Step 2: We show that $F^{*}$ can be compromised. Let $N=\{1,2,3\}$. Let pair $V, V^{\prime} \in \mathcal{S}^{N}$ be defined as follows: $v_{1}=0.2, v_{1}^{\prime}=0.3$, and $v_{2}=v_{2}^{\prime}=v_{3}=v_{3}^{\prime}=0$. Hence, $r_{V}^{*}=r_{V_{1}}(0)=0.4$ and $r_{V^{\prime}}^{*}=r_{V_{1}^{\prime}}(0)=0.6$. Therefore, $F(V)=[0,0.4]$ and $F\left(V^{\prime}\right)=[0,0.6]$. Clearly, $F$ is compromised.

We conclude this section by presenting the "efficient versions" of Theorems 3.1 and 3.2. Notice that now peaks-onliness and $\min / \max$ continuity become substitutable.

Theorem 3.3. The following four statements for a correspondence $F \in \mathcal{F}$ are equivalent.

(i) F satisfies strategy-proofness, peaks-onliness, and voter-sovereignty.

(ii) F satisfies uncompromisingness and voter-sovereignty.

(iii) $F$ satisfies strategy-proofness, $\min / \max$ continuity, and votersovereignty.

(iv) $F$ is an efficient generalized median correspondence. 
Proof. The equivalence of statements (i) and (ii) follows from Proposition 3.4. The equivalence of statement (ii) and (iv) is shown as follows. By Theorem 3.1, statement (ii) implies $F \in \mathcal{F}_{G}$. Hence, by the definition of $\mathcal{F}_{G}, F$ satisfies peaksonliness. Thus, Proposition 3.3 and the definition of $\mathcal{F}_{E G}$ imply $F \in \mathcal{F}_{E G}$, i.e., statement (ii) implies statement (iv). Moreover, by Theorem 3.1 and $\mathcal{F}_{E G} \subsetneq$ $\mathcal{F}_{G}$, statement (iv) implies $F$ satisfies uncompromisingness; in addition, by the definition of $\mathcal{F}_{E G}$, statement (iv) implies $F$ satisfies efficiency and therefore votersovereignty, i.e., statement (iv) implies statement (ii). Finally, notice that this equivalence of statements (ii) and (iv) also holds in domain $\mathcal{S}$.

Next, by Proposition 3.6, statement (iii) implies statement (ii). We complete the proof by showing statement (ii) implies statement (iii). By Step 1 of the proof of Theorem 3.1(statement (ii) implies statement (iii)) on page 106, if $F \in \mathcal{F}$ satisfies uncompromisingness, then for $V \in \mathcal{R}^{N}$ and each $i \in N$ the following holds. If $V_{i}^{0}$ is such that $v_{i}^{0}=0$ and $V_{i}^{1}$ is such that $v_{i}^{1}=1$, then $\underline{F}(V)=\operatorname{med}\left(\underline{F}(V), \underline{F}\left(V_{-i}, V_{i}^{0}\right), \underline{F}\left(V_{-i}, V_{i}^{1}\right)\right)$ and $\bar{F}(V)=$ $\operatorname{med}\left(\bar{F}(V), \bar{F}\left(V_{-i}, V_{i}^{0}\right), \bar{F}\left(V_{-i}, V_{i}^{1}\right)\right)$. Hence clearly statement (ii) implies $F$ satisfies $\min / \max$ continuity. Finally, by the equivalence of statements (i) and (ii), statement (ii) implies $F$ satisfies strategy-proofness.

Theorem 3.4. The following four statements for a correspondence $F \in \mathcal{F}$ are equivalent.

(i) F satisfies strategy-proofness, peaks-onliness, anonymity, and votersovereignty.

(ii) F satisfies uncompromisingness, anonymity, and voter-sovereignty.

(iii) F satisfies strategy-proofness, $\min / \max$ continuity, anonymity, and voter-sovereignty.

(iv) $F$ is an efficient median correspondence.

Proof. The equivalence of statements (i), (ii), and (iii) follows from Theorem 3.3. The equivalence of statements (ii) and (iv) is shown as follows. By Theorem 3.2, statement (ii) implies $F \in \mathcal{F}_{M}$. Hence, Proposition 3.3 and the definition of $\mathcal{F}_{E M}$ imply $F \in \mathcal{F}_{E M}$, i.e., statement (ii) implies statement (iv). Moreover, by Theorem 3.2 and $\mathcal{F}_{E M} \subsetneq \mathcal{F}_{M}$, statement (iv) implies $F$ satisfies uncompromisingness, and anonymity; in addition, by the definition of $\mathcal{F}_{E M}$, statement (iv) implies $F$ satisfies efficiency and therefore voter-sovereignty, i.e., statement (iv) implies statement (ii). Finally, notice that this equivalence of statements (ii) and (iv) also holds in domain $\mathcal{S}$. 


\subsubsection{Results in the single-peaked and symmetric domain $\mathcal{S}$}

We now show the characterizations in domain $\mathcal{S}$ that are -loosely speaking- equivalent to those presented in Section 3.5.2 for domain $\mathcal{R}$. Specifically, the non-efficient characterizations in domain $\mathcal{R}$ (Theorems 3.1 and 3.2) cannot be extended in domain $\mathcal{S}$. This is illustrated by the counter-example presented on page 84 , where the given correspondence satisfies strategy-proofness, anonymity, and $\min / \max$ continuity in domain $\mathcal{S}$ but violates uncompromisingness. This violation is of importance because as shown by the proof of Theorem 3.1 (statement (iii) implies statement (ii)) on page 103 -this part of the proof also holds in domain $\mathcal{S}$ - both classes of generalized median correspondences and median correspondences satisfy uncompromisingness.

Concerning the efficient characterizations in domain $\mathcal{R}$ (Theorems 3.3 and 3.4), these do extend in domain $\mathcal{S}$. Moreover, since peaks-onliness is an inherent property of domain $\mathcal{S}, \min / \max$ continuity is unnecessary in these characterizations.

Theorem 3.5. The following three statements for a correspondence $F \in \mathcal{F}$ are equivalent.

(i) F satisfies strategy-proofness and voter-sovereignty.

(ii) F satisfies uncompromisingness and voter-sovereignty.

(iii) $F$ is an efficient generalized median correspondence.

Proof. The equivalence of statements (i) and (ii) constitutes Proposition 3.5. The equivalence of statements (ii) and (iii) follows from Theorem 3.3 (recall that as noted on page 85, statements (ii) and (iv) of Theorem 3.3 are also equivalent in domain $\mathcal{S}$ ).

Theorem 3.6. The following three statements for a correspondence $F \in \mathcal{F}$ are equivalent.

(i) F satisfies strategy-proofness, voter-sovereignty, and anonymity.

(ii) F satisfies uncompromisingness, voter-sovereignty, and anonymity.

(iii) $F$ is an efficient median correspondence.

Proof. The equivalence of statements (i) and (ii) constitutes Proposition 3.5. The equivalence of statements (ii) and (iii) follows from Theorem 3.4 (recall that as noted on page 85, statements (ii) and (iv) of Theorem 3.4 are also equivalent in domain $\mathcal{S})$. 


\subsubsection{Independence of properties}

Concerning the independence of the properties used in our results, consider the following four correspondences. First, correspondence $F^{*}$ proposed by the counterexample on page 84. Second, let correspondence $F_{1} \in \mathcal{F}$ choose the minimum announced peak when more than two agents prefer it against the maximum announced peak, and choose the maximum announced peak otherwise; in both domains $\mathcal{R}$ and $\mathcal{S}$, it satisfies strategy-proofness, anonymity, and voter-sovereignty but violates peaks-onliness (in domain $\mathcal{R}$ ), $\min / \max$ continuity, and uncompromisingness. Third, for a small and positive value $\varepsilon$ let correspondence $F_{2} \in \mathcal{F}$ choose the minimum of: (a) the minimum announced peak plus $\varepsilon$ and (b) the maximum peak; in both domains $\mathcal{R}$ and $\mathcal{S}$, it satisfies all the properties we are interested in except for strategy-proofness and uncompromisingness. Finally, let $F_{3} \in \mathcal{F}$ be the "constant" correspondence always choosing 0 ; in both domains $\mathcal{R}$ and $\mathcal{S}$, it satisfies all the properties we are interested in except for voter-sovereignty.

Table 3.1 summarizes our results and also depicts that correspondences $F^{*}$, $F^{1}, F^{2}$, and $F^{3}$ suffice to show the independence of the properties used in our results.

\begin{tabular}{|c|c|c|c|c|c|c|c|c|}
\hline & $\mathcal{F}_{G}$ & $\mathcal{F}_{E G}$ & $\mathcal{F}_{M}$ & $\mathcal{F}_{E M}$ & $F^{*}$ & $F_{1}$ & $F_{2}$ & $F_{3}$ \\
\hline Domain $\mathcal{R} \bigcirc$ & (1) (2) & (1) (2) (3) & (1) (2) & (1) (2) (3) & & & & \\
\hline Domain $\mathcal{S} \bullet$ & & 45 & & 45 & & & & \\
\hline Strategy-proofness & (1) & (1) (2) & (1) & (1) (2) & $\checkmark$ & $\checkmark$ & $x$ & $\checkmark$ \\
\hline Peaks-onliness (in $\mathcal{R}$ ) & (1) & & (1) & (1) & $x$ & $x$ & $\checkmark$ & $\checkmark$ \\
\hline Min/max continuity & & (2) & & (2) & $\checkmark$ & $x$ & $\checkmark$ & $\checkmark$ \\
\hline Voter-sovereignty & & (1) (2) (3) 45 & & (1) (2) (3) 45 & $x$ & $\checkmark$ & $\checkmark$ & $x$ \\
\hline Anonymity & & & (1) (2) & (1) (2) (3) 45 & $\checkmark$ & $\checkmark$ & $\checkmark$ & $\checkmark$ \\
\hline Uncompromisingness & (2) & (3) & (2) & (5) & $x$ & $x$ & $x$ & $\checkmark$ \\
\hline
\end{tabular}

The table above has a double purpose. First, columns $\mathcal{F}_{G}, \mathcal{F}_{E G}, \mathcal{F}_{M}$, and $\mathcal{F}_{E M}$, denoting the characterized classes of correspondences, defined in Sections 3.3.1 and 3.3.2, summarize our characterization results, with all results in domain $\mathcal{R}$ shown by circled black numbers in a white background and all results in domain $\mathcal{S}$ shown by circled white numbers in a black background. For example, in the column referring to $\mathcal{F}_{E G}$, the white circles containing number 4 show that in domain $\mathcal{S}$, strategyproofness and voter-sovereignty characterize this class of correspondences. Second, the table shows the independence of the properties used in each characterization result. Specifically, all combinations of properties that must be checked are satisfied by at least one of the four non-median correspondences $F^{*}, F_{1}, F_{2}$, and $F_{3}$, defined in Section 3.5.4 (with the exception of the combinations showing the independence of anonymity in the characterizations of $\mathcal{F}_{M}$ and $\mathcal{F}_{E M}$; these combinations are satisfied directly by the characterizations of $\mathcal{F}_{G}$ and $\mathcal{F}_{E G}$ respectively).

Table 3.1: Summary of the main results and independence of properties 


\subsubsection{Discussion}

The classes of generalized median correspondences and median correspondences characterized in this chapter seem very similar to the class of double median functions characterized in Heo (2013). Moreover, the reader might have noticed that the left-peaks (right-peaks) function, characterized in Miyagawa (2001), resembles a special case of a median correspondence. However, there are some notable differences between these results and the results of this paper, which are summarized in the following three points.

(i) By using the max-extension of preferences in our setting, as is the case in the two aforementioned papers, it is straightforward to show that the only efficient correspondence would be the one always choosing the interval of the peaks, that is, for all $V \in \mathcal{R}, F(V)=[\underline{v}, \bar{v}]$. This follows from the agents only caring about their best alternative in a set, and from our setting not limiting the number of alternatives that may be chosen at a given profile.

(ii) Concerning the results of Heo (2013), the class of double median functions seems to be equivalent to the class of median correspondences. Specifically, the pair of alternatives $\left(x_{1}, x_{2}\right)$ chosen by a double median function are essentially the minimum and maximum alternatives of the interval chosen by a median correspondence. However, apart from the distinction made in point (i), we should note the following: Heo's characterization result makes use of users-only, a property that partitions each coalition of agents into two sub-coalitions; one preferring $x_{1}$ over $x_{2}$, and everyone else, with only the first partition (second partition) influencing the choice of alternative $x_{1}\left(x_{2}\right)$. In our setting, for each $F^{a, b} \in \mathcal{F}_{M}$, the choice of both vectors $a$ and $b$ depends on the preferences of all the agents.

(iii) Concerning the results of Miyagawa (2001) and apart of the distinction made in point (i), although the left-peaks function seems to be a special case of a median correspondence, this is not the case; the left-peaks function always chooses the two distinct left-most peaks, and moreover, the setting of Miyagawa requires that at least two distinct peaks exist in each profile. In our setting, the median correspondence that looks "similar" to the left-peaks function is $F^{a, b} \in \mathcal{F}$ where $a=(0, \ldots, 0)$ and $b=(0, \ldots, 0,1)$. Although this correspondence seems to choose the two left-most peaks, when two or more agents share the minimum peak, it only chooses the minimum peak. Furthermore, it should be noted that in Chapter 2 the same properties with Miyagawa (namely, efficiency and replacement-dominance) are considered, under (almost) the same setting as in this chapter. There, it is shown that each correspondence satisfying said properties is essentially a function, 
reconfirming the characterization of Vohra (1999) for (fixed population) targetpoint functions. ${ }^{26}$ In addition, after weakening replacement-dominance to onesided replacement-dominance ${ }^{27}$, (fixed population) target set correspondences ${ }^{28}$ are characterized, a sub-class of efficient median correspondences which -as discussed above- does not include either the left-peaks rule or the right-peaks rule.

\section{A On $\min / \max$ continuity}

In the sequel, all properties are defined for correspondences in domain $\mathcal{R}$ but also apply to correspondences in domain $\mathcal{S}$. Moreover, all results presented in this section, hold in both domains.

Upper-hemi continuity. For each $V \in \mathcal{R}^{N}$, each $i \in N$, each $\left\{V_{i}^{k}\right\}$ in $\mathcal{R}$ such that $V_{i}^{k} \rightarrow V_{i}$, and each $\left\{x^{k}\right\}$ in $A$ such that $x^{k} \rightarrow x$, the following holds. If for each $k \in \mathbb{N}^{+}, x^{k} \in F\left(V_{-i}, V_{i}^{k}\right)$, then $x \in F(V)$.

Lower-hemi continuity. For each $V \in \mathcal{R}^{N}$, each $i \in N$, and each $\left\{V_{i}^{k}\right\}$ in $\mathcal{R}$ such that $V_{i}^{k} \rightarrow V_{i}$, the following holds. If $x \in F(V)$, then there exists $\left\{x^{k}\right\}$ in $A$ such that $x^{k} \rightarrow x$ and for each $k \in \mathbb{N}^{+}, x^{k} \in F\left(V_{-i}, V_{i}^{k}\right)$.

Lemma 3.5. The following two statements for correspondences are equivalent.

(i) Min/max continuity is satisfied.

(ii) Upper-hemi continuity and lower-hemi continuity are satisfied.

Proof of Lemma 3.5. Let $F \in \mathcal{F}$. If $F$ satisfies upper-hemi continuity and lower-hemi continuity then trivially, it also satisfies $\min / \max$ continuity. Next, let $F$ satisfy $\min / \max$ continuity, $V \in \mathcal{R}^{N}$, and $\left\{V_{i}^{k}\right\}$ be in $\mathcal{R}$. We show that $F$ satisfies upper-hemi continuity and lower-hemi continuity in two steps.

Step 1. We show $F$ satisfies upper-hemi continuity. Let $\left\{x^{k}\right\}$ in $A$ such that $x^{k} \rightarrow x$ and for each $k \in \mathbb{N}^{+}, x_{k} \in F\left(V_{-i}, V_{i}^{k}\right)$. Hence, for each $k \in \mathbb{N}^{+}$, $\underline{F}\left(V_{-i}, V_{i}^{k}\right) \leq x^{k} \leq \bar{F}\left(V_{-i}, V_{i}^{k}\right)$. Moreover, by min/max continuity, $\underline{F}\left(V_{-i}, V_{i}^{k}\right) \rightarrow$ $\underline{F}(V)$ and $\bar{F}\left(V_{-i}, V_{i}^{k}\right) \rightarrow \bar{F}(V)$, which implies $\underline{F}(V) \leq x \leq \bar{F}(V)$ since otherwise, $\min / \max$ continuity would imply that there exists $k^{*} \in \mathbb{N}^{+}$such that $x_{k^{*}} \notin$ $F\left(V_{-i}, V_{i}^{k^{*}}\right)$. Therefore, $x \in F(V)$.

Step 2. We show $F$ satisfies lower-hemi continuity. Let $x \in F(V)$ and $V_{i}^{k} \rightarrow$ $V_{i}$. Hence, for all $k \in \mathbb{N}^{+}, \underline{F}\left(V_{-i}, V_{i}^{k}\right) \leq \bar{F}\left(V_{-i}, V_{i}^{k}\right)$ and by $\min / \max$ continuity,

\footnotetext{
${ }^{26}$ Each target point function is determined by its target point: if the target point is efficient, it is chosen; if it is not efficient, the closest efficient point is chosen.

${ }^{27}$ See page 38 for a formal definition.

${ }^{28}$ See page 33 for a formal definition.
} 
$\left[\underline{F}\left(V_{-i}, V_{i}^{k}\right) \rightarrow \underline{F}(V)\right.$ and $\left.\bar{F}\left(V_{-i}, V_{i}^{k}\right) \rightarrow \bar{F}(V)\right]$. It follows that there exists $\left\{x^{k}\right\}$ in $A$ such that $x^{k} \rightarrow x$ and for each $k \in \mathbb{N}^{+}, \underline{F}\left(V_{-i}, V_{i}^{k}\right) \leq x^{k} \leq \bar{F}\left(V_{-i}, V_{i}^{k}\right)$.

\section{B Proofs of Propositions 3.2 and 3.3}

Before proceeding to the proofs of Propositions 3.2 and 3.3, we show the following. When strategy-proofness is satisfied, voter sovereignty is equivalent with unanimity; a property stronger than voter-sovereignty but weaker than efficiency, that requires when all agents announce the same peak, only this peak to be chosen. ${ }^{29}$ This result holds in both domains.

Lemma 3.6. For strategy-proof correspondences, voter-sovereignty and unanimity are equivalent.

Proof. Let $F \in \mathcal{F}$. Trivially, unanimity implies voter sovereignty. Hence, let $F$ satisfy strategy-proofness and voter-sovereignty. We show that $F$ satisfies unanimity.

Let $a \in A$ and $R \in \mathcal{R}^{N}$ be such that $p=(a, \ldots, a)$. By voter-sovereignty, there exists $V \in \mathcal{R}^{N}$ such that $F(V)=a$. Let $M \subseteq N$ contain all the agents in $N$ whose announced peak at $V$ is not $a$, i.e., for each $i \in M, v_{i} \neq a$, and for each $j \in N \backslash M, v_{j}=p_{j}=a$. Without loss of generality, index the agents in $N$ such that $M=\{1, \ldots, m\}$. Next, consider profile $V^{1}=\left(V_{-1}, R_{1}\right)$. By strategyproofness, $F\left(V^{1}\right) R_{1} F(V)$. Hence, single-peakedness and $F(V)=a=p_{1}$ imply $F\left(V^{1}\right)=a$. Finally, for each $k=\{2, \ldots, m\}$ in increasing indexing order, consider profile $V^{k}=\left(V_{-k}^{k-1}, R_{k}\right)$. By the arguments presented for $V^{1}, F\left(V^{k}\right)=F(V)=a$. Therefore, since $V^{m}=R, F\left(V^{m}\right)=F(R)=a$.

We proceed with the proof of Proposition 3.2, which makes use of Lemma 3.6. This proof holds only in domain $\mathcal{S}$ because it makes use of the inherent peaksonliness of this domain.

Proof of Proposition 3.2. Let $F \in \mathcal{F}$ satisfy strategy-proofness. The equivalence of unanimity and voter-sovereignty follows by Lemma 3.6. In addition, it is trivial to show that efficiency implies unanimity. Therefore, it remains to show that unanimity implies efficiency. We do so by contradiction; specifically, we show that if $F(V) \notin \mathrm{E}(V)$, then unanimity is violated.

\footnotetext{
${ }^{29}$ Formally, given $F \in \mathcal{F}$, for each $V \in \mathcal{R}^{N}$ such that $v=(x, \ldots, x), F(V)=\{x\}$.
} 
Let $V \in \mathcal{S}^{N}$ and without loss of generality, assume $v_{1} \leq \cdots \leq v_{n}$. By Proposition 3.1, there are two cases.

Case 1. Let $\bar{v}<\bar{F}(V)$. For all agents $i \in N$, define $V_{i}^{\prime} \in \mathcal{S}$ to be such that $v_{i}^{\prime}=v_{n}$.

First, consider agent 1 , where $\underline{v}=v_{1} \leq \bar{v}<\bar{F}(V)$. By single-peakedness, either $\left[\bar{F}(V) \in w_{V_{1}}(F(V))\right.$ and thus $\left.w_{V_{1}}(F(V)) \notin \mathrm{E}(V)\right]$ or $\left[\underline{F}(V)=w_{V_{1}}(F(V))\right.$ and thus $v_{1}=\underline{v}<\bar{F}(V)$ and single-peakedness imply $\underline{F}(V)<\underline{v}$, and therefore, $\left.w_{V_{1}}(F(V)) \notin \mathrm{E}(V)\right]$. Next, recall the indifference relation $r_{V_{i}}$ and let $x_{1}=$ $r_{V_{1}}\left(w_{V_{1}}(F(V))\right) .{ }^{30}$ If $\bar{F}(V) \in w_{V_{1}}(F(V))$, then $v_{1}<\bar{F}(V)$ and single-peakedness imply $x_{1}<v_{1}=\underline{v}$ and hence, $x_{1} \notin \mathrm{E}(V)$. Similarly, if $\underline{F}(V)=w_{V_{1}}(F(V))$, then $\underline{F}(V)<\underline{v} \leq \bar{v}<\bar{F}(V)$ and single-peakedness imply $x_{1}>\bar{v}$ and hence, $x_{1} \notin \mathrm{E}(V)$. Finally, assume $R_{1}=V_{1}$ and consider $V^{1}=\left(V_{-1}, V_{1}^{\prime}\right)$. By strategyproofness, $w_{R_{1}}(F(V)) R_{1} w_{R_{1}}\left(F\left(V^{1}\right)\right)$; hence, $w_{V_{1}}(F(V)) \notin \mathrm{E}(V), x_{1} \notin \mathrm{E}(V)$, and single-peakedness implies $w_{R_{1}}\left(F\left(V^{1}\right)\right) \notin \mathrm{E}(V)$. Then, since $\mathrm{E}\left(V^{1}\right) \subseteq \mathrm{E}(V)$ implies $w_{R_{1}}\left(F\left(V^{1}\right)\right) \notin \mathrm{E}\left(V^{1}\right)$; and thus, $F\left(V^{1}\right) \notin \mathrm{E}\left(V^{1}\right)$.

Next, consider agent 2 at profile $V^{1}$ and recall that $w_{R_{1}}\left(F\left(V^{1}\right)\right) \notin \mathrm{E}\left(V^{1}\right)$ and $x_{1} \notin \mathrm{E}(V)$. Let $x^{2}=r_{V_{2}}\left(w_{V_{2}}\left(F\left(V^{1}\right)\right)\right)$. If $\underline{F}\left(V^{1}\right) \subseteq w_{R_{1}}\left(F\left(V^{1}\right)\right)$, then $\underline{F}\left(V^{1}\right)<$ $v_{1}$; hence, $v_{1} \leq v_{2}$, single-peakedness, and $V_{2} \in \mathcal{S}$ imply $\underline{F}\left(V^{1}\right) \subseteq w_{V_{2}}\left(F\left(V^{1}\right)\right)$ and $x^{2} \geq \bar{F}(V) .{ }^{31}$ Thus, $w_{V_{2}}\left(F\left(V^{1}\right)\right), x_{2} \notin \mathrm{E}\left(V^{1}\right)$. If $\bar{F}\left(V^{1}\right)=w_{R_{1}}\left(F\left(V^{1}\right)\right)$, then $\bar{v}^{1}<\bar{F}\left(V^{1}\right)$; hence, $v_{2} \leq \bar{v}^{1}$ and single-peakedness imply either $\left[\bar{F}\left(V^{1}\right) \subseteq\right.$ $w_{V_{2}}\left(F\left(V^{1}\right)\right)$ and $\left.x_{2}<\underline{v}_{1}\right]$ or $\left[\underline{F}\left(V^{1}\right)=w_{V_{2}}\left(F\left(V^{1}\right)\right)\right.$ and $\left.\bar{v}^{1}<\bar{F}\left(V^{1}\right)<x_{2}\right]$. Thus, $w_{V_{2}}\left(F\left(V^{1}\right)\right), x_{2} \notin \mathrm{E}\left(V^{1}\right)$. Therefore, by the arguments presented for $V^{1}, F\left(V^{2}\right) \notin$ $\mathrm{E}\left(V^{2}\right)$.

Finally, for each $k \in\{3, \ldots, n-1\}$, in increasing order, consider profile $V^{k}=$ $\left(V_{-k}^{k-1}, V_{k}^{\prime}\right)$. By the arguments presented for agents 1 and 2 above, $F\left(V^{k}\right) \notin \mathrm{E}\left(V^{k}\right)$. Therefore, at profile $V^{n-1}$ where $v^{n-1}=\left(v_{n}, \ldots, v_{n}\right), F\left(V^{n-1}\right) \notin \mathrm{E}\left(V^{n-1}\right)$ implying $F\left(V^{n-1}\right) \neq\left\{v_{n}\right\}$ which contradicts unanimity.

Case 2. Let $\underline{v}>\underline{F}(V)$. The proof is symmetric to Case 1 .

Notice that although for didactic reasons Proposition 3.3 proceeds Proposition 3.4 in the main text, the proof of Proposition 3.3 makes use of Proposition 3.4 (proof in Appendix 3.C). Recall that this result holds in both domains.

\footnotetext{
${ }^{30}$ To be precise, if agent 1 has two worst points on $F(V)$, then with some abuse of notation, assume $w_{V_{1}}(F(V))$ is the smallest of the two worst points, which implies that $x_{1}$ is then the largest of the two worst points.

${ }^{31}$ To be precise, if agent 2 has two worst points on $F\left(V^{1}\right)$, then with some abuse of notation, assume $w_{V_{2}}\left(F\left(V^{1}\right)\right)$ is the smallest of the two worst points, which implies that $x_{2}$ is then the largest of the two worst points.
} 
Proof of Proposition 3.3. Let $F \in \mathcal{F}$ satisfy strategy-proofness. By Lemma 3.6, unanimity and voter-sovereignty are equivalent. In addition, it is easy to show that efficiency implies unanimity.

The proof proceeds in three steps. In Step 1 we show that if in addition to strategy-proofness, $F$ satisfies peaks-onliness and unanimity, the following holds. Given an announced profile where an efficient set is chosen, if an agent with the minimum -but not unique- announced peak changes his announcement by moving his announced peak to the right, an efficient set is chosen again. Step 2 shows the same result but for the case where in addition to strategy-proofness, $F$ satisfies $\min / \max$ continuity and unanimity. Finally in Step 3, by unanimity and the intermediate results of Steps 1 and 2, we show that $F$ satisfies efficiency.

Step 1. In addition to strategy-proofness, let $F$ satisfy peaks-onliness and unanimity. By Proposition 3.4, $F$ also satisfies uncompromisingness. Let $V \in \mathcal{R}^{N}$ and $i \in N$ be such that $F(V) \in \mathrm{E}(V)$ and $v_{i}=\underline{v}$ but where $i$ does not have the unique minimum peak. Hence, by Proposition 3.1, $F(V) \subseteq \operatorname{Conv}(v)$. In addition, let $V_{i}^{\prime} \in \mathcal{R}$ be such that $v_{i}^{\prime} \geq \bar{v}$. Assuming $\underline{F}\left(V_{-i}, V_{i}^{\prime}\right)<\underline{v}$ implies $\underline{F}\left(V_{-i}, V_{i}^{\prime}\right)<v_{i} \leq v_{i}^{\prime}$. Hence, by uncompromisingness, $\underline{F}\left(V_{-i}, V_{i}^{\prime}\right)=\underline{F}(V)<\underline{v}$, which contradicts $F(V) \in \mathrm{E}(V)$. Similarly, assuming $\bar{F}\left(V_{-i}, V_{i}^{\prime}\right)>v_{i}^{\prime}$ implies $\bar{F}\left(V_{-i}, V_{i}^{\prime}\right)>v_{i}^{\prime} \geq v_{i}$. Hence, by uncompromisingness, $\bar{F}\left(V_{-i}, V_{i}^{\prime}\right)=\bar{F}(V)>\bar{v}$, which contradicts $F(V) \in \mathrm{E}(V)$. Therefore, $F\left(V_{-i}, V_{i}^{\prime}\right) \in \mathrm{E}(V)$.

Step 2. In addition to strategy-proofness, let $F$ satisfy $\min / \max$ continuity and unanimity. Define $V \in \mathcal{R}^{N}$ and $V_{i}^{\prime} \in \mathcal{R}$ as in Step 1. By single-peakedness, $w_{V_{i}^{\prime}}(F(V))=\underline{F}(V)$. We show that $F\left(V_{-i}, V_{i}^{\prime}\right) \in \mathrm{E}\left(V_{-i}, V_{i}^{\prime}\right)$ by discrediting all three cases where $F\left(V_{-i}, V_{i}^{\prime}\right) \notin \mathrm{E}\left(V_{-i}, V_{i}^{\prime}\right)$.

Case 1. Let $\underline{F}\left(V_{-i}, V_{i}^{\prime}\right)<\underline{v}$. In addition, let $R_{i}=V_{i}^{\prime}$. By single-peakedness, $\underline{F}(V) P_{i} \underline{F}\left(V_{-i}, V_{i}^{\prime}\right)$, hence $w_{R_{i}}(F(V))=\underline{F}(V)$ implies $w_{R_{i}}(F(V)) P_{i} w_{R_{i}}\left(F\left(V_{-i}, V_{i}^{\prime}\right)\right)$. Therefore, if at profile $\left(V_{-i}, V_{i}^{\prime}\right)$ agent $i$ deviates by announcing $V_{i}$, his worst point improves. This contradicts strategy-proofness.

Case 2. Let $\underline{F}\left(V_{-i}, V_{i}^{\prime}\right)>v_{i}^{\prime}$. Since $\underline{F}(V) \leq \bar{F}(V) \leq v_{i}^{\prime}$, by min/max continuity, there exists some profile $V_{i}^{\prime \prime} \in \mathcal{R}$ such that $V_{i}^{\prime \prime} \neq V_{i}^{\prime}$ and $v_{i}^{\prime} \in F\left(V_{-i}, V_{i}^{\prime \prime}\right)$. Let $R_{i}=V_{i}^{\prime}$; hence, $b_{R_{i}}\left(F\left(V_{-i}, V_{i}^{\prime \prime}\right)\right)=v_{i}^{\prime}$ implying $b_{R_{i}}\left(F\left(V_{-i}, V_{i}^{\prime \prime}\right)\right) P_{i} b_{R_{i}}\left(F\left(V_{-i}, V_{i}^{\prime}\right)\right)$. Therefore, if at profile $\left(V_{-i}, V_{i}^{\prime}\right)$ agent $i$ deviates by announcing $V_{i}^{\prime \prime}$, his best point improves. This contradicts strategy-proofness.

Case 3. Let $\underline{v} \leq \underline{F}\left(V_{-i}, V_{i}^{\prime}\right) \leq v_{i}^{\prime}$ and $\bar{F}\left(V_{-i}, V_{i}^{\prime}\right)>v_{i}^{\prime}$. In the following, we describe a series of actions that when performed in sequence construct -after a finite number of "moves" - profile $V^{\prime}$, such that $v^{\prime}=\left(v_{i}^{\prime}, \ldots, v_{i}^{\prime}\right)$ and $F\left(V^{\prime}\right) \neq v_{i}^{\prime}$, i.e., a profile at which unanimity is violated. 
Action 1. Let profile $V^{0}=\left(V_{-i}, V_{i}^{\prime}\right)$. Let $N_{1} \subsetneq N$ be such that $j \in N_{1}$ if and only if $v_{j}^{0} \neq v_{i}^{\prime}$ and $v_{j}^{0}<\underline{F}\left(V^{0}\right)$. If $N_{1}=\emptyset$, then proceed to Action 2. Otherewise, let $j \in N_{1}$. By $v_{j}^{0}<\underline{F}\left(V^{0}\right) \leq \bar{F}\left(V^{0}\right)$ and single-peakedness, $b_{V_{j}^{0}}\left(F\left(V^{0}\right)\right)=\underline{F}\left(V^{0}\right)$ and $w_{V_{j}^{0}}\left(F\left(V^{0}\right)\right)=\bar{F}\left(V^{0}\right)$. Let $V_{j}^{1}=V_{i}^{\prime}$ and profile $V^{1}=$ $\left(V_{-j}^{0}, V_{j}^{1}\right)$. Assume $R_{j}=V_{j}^{0}$. By strategy-proofness, $b_{R_{j}}\left(F\left(V^{0}\right)\right) R_{j} b_{R_{j}}\left(F\left(V^{1}\right)\right)$ and $w_{R_{j}}\left(F\left(V^{0}\right)\right) R_{j} w_{R_{j}}\left(F\left(V^{1}\right)\right)$; hence, by single-peakedness, either $\left[\underline{F}\left(V^{0}\right) \leq \underline{F}\left(V^{1}\right)\right.$ and $\left.\bar{F}\left(V^{0}\right) \leq \bar{F}\left(V^{1}\right)\right]$ or $\left[\underline{F}\left(V^{1}\right) \leq \bar{F}\left(V^{1}\right)<v_{j}^{0}\right]$. However, if $\underline{F}\left(V^{1}\right) \leq \bar{F}\left(V^{1}\right)<v_{j}^{0}$, then by $\min / \max$ continuity there exist preference $V_{j}^{*} \in \mathcal{R}$ such that $v_{j}^{0} \in$ $F\left(V_{-j}^{0}, V_{j}^{*}\right)$. This violates strategy-proofness since if at profile $V^{0}$ agent $j$ deviates by announcing $V^{*}$, his best point improves. Hence, $\underline{F}\left(V^{0}\right) \leq \underline{F}\left(V^{1}\right)$ and $\bar{F}\left(V^{0}\right) \leq \bar{F}\left(V^{1}\right)$. Therefore, $v_{i}^{\prime}<\bar{F}\left(V^{1}\right)$.

Next, let $N_{2} \subsetneq N$ be such that $k \in N_{2}$ if and only if $v_{k}^{1} \neq v_{i}^{\prime}$ and $v_{k}^{1}<\underline{F}\left(V^{1}\right)$. If $N_{2}=\emptyset$, then proceed to Action 2. Otherwise, let $k \in N_{2}$. In addition, let $V_{k}^{2}=V_{i}^{\prime}$ and profile $V^{2}=\left(V_{-k}^{1}, V_{k}^{2}\right)$. By the process described in the previous paragraph for agent $j, v_{i}^{\prime}<\bar{F}\left(V^{2}\right)$.

Finally, repeat this process $\mu$ times (where $\mu$ is smaller than the number of agents, $\mu \leq n-1)$ until the following holds. Set $N_{\mu} \subsetneq N$, constructed similarly to $N_{1}$ and $N_{2}$, is empty. When this occurs, proceed to Action 2.

Action 2. Let profile $\bar{V}^{0}=V^{\mu-1}$. Let $\bar{N}_{1} \subsetneq N$ be such that $j \in \bar{N}_{1}$ if and only if $\bar{v}_{j}^{0} \neq v_{i}^{\prime}$ and $\bar{F}\left(\bar{V}^{0}\right) \subseteq w_{\bar{V}_{j}^{0}}\left(F\left(\bar{V}^{0}\right)\right)$. Recall that $\bar{F}\left(\bar{V}^{0}\right)>v_{i}^{\prime}$. If $\bar{N}_{1}=\emptyset$, then proceed to Action 3. Otherwise, let $j \in \bar{N}_{1}$ and notice that by $N_{\mu}=\emptyset$ (as defined in Action 1), the choice of $\bar{N}_{1}$ implies $\underline{F}\left(\bar{V}^{0}\right) \leq \bar{v}_{j}^{0}<\bar{F}\left(\bar{V}^{0}\right)$. Define $\bar{V}_{j}^{1} \in \mathcal{R}$ such that $\bar{v}_{j}^{1}=v_{i}^{\prime}$ and $w_{\bar{V}_{j}^{1}}\left(F\left(\bar{V}^{0}\right)\right)=\underline{F}\left(\bar{V}^{0}\right)$, and let profile $\bar{V}^{1}=\left(\bar{V}_{-j}^{0}, \bar{V}_{j}^{1}\right)$. Assume that $R_{j}=\bar{V}_{j}^{0}$. By strategy-proofness, $w_{R_{j}}\left(F\left(\bar{V}^{0}\right)\right) R_{j} w_{R_{j}}\left(F\left(\bar{V}^{1}\right)\right)$; hence, by single-peakedness, $\bar{F}\left(\bar{V}^{0}\right) \leq \bar{F}\left(\bar{V}^{1}\right)$ and perhaps, $\underline{F}\left(\bar{V}^{0}\right)>\underline{F}\left(\bar{V}^{1}\right)$. Assume that $R_{j}=\bar{V}_{j}^{1}$. If $\underline{F}\left(\bar{V}^{1}\right)<\underline{F}\left(\bar{V}^{0}\right)<\bar{v}_{j}^{1}$, then single-peakedness implies $w_{R_{j}}\left(F\left(\bar{V}^{0}\right)\right) P_{j}$ $w_{R_{j}}\left(F\left(\bar{V}^{1}\right)\right)$. This violates strategy-proofness since if at profile $\bar{V}^{1}$ agent $j$ deviates by announcing $\bar{V}_{j}^{0}$, his worst point improves. Therefore, $\bar{F}\left(\bar{V}^{0}\right) \leq \bar{F}\left(\bar{V}^{1}\right)$ and $\underline{F}\left(\bar{V}^{0}\right) \leq \underline{F}\left(\bar{V}^{1}\right)$. Hence, $v_{i}^{\prime}<\bar{F}\left(\bar{V}^{1}\right)$.

Next, if $\underline{F}\left(\bar{V}^{0}\right)<\underline{F}\left(\bar{V}^{1}\right)$, perhaps there exist some agents $\bar{j} \in N$ such that $v_{\bar{j}}<\underline{F}\left(\bar{V}^{1}\right) \leq \bar{F}\left(\bar{V}^{1}\right)$. If this is the case, then repeat the process described in Action 1 and denote the resulting profile (again) by $\bar{V}^{1}$. If no such agents exist, then $\bar{V}^{1}$ is the profile constructed in the end of the previous paragraph.

Following this, let $\bar{N}_{2} \subsetneq N$ be such that $k \in \bar{N}_{2}$ if and only if $\bar{v}_{k}^{1} \neq v_{i}^{\prime}$ and $\bar{F}\left(\bar{V}^{1}\right) \subseteq w_{\bar{V}_{k}^{1}}\left(F\left(\bar{V}^{1}\right)\right)$, where $\bar{F}\left(\bar{V}^{1}\right)>v_{i}^{\prime}$. If $\bar{N}_{2}=\emptyset$, then proceed to Action 3 . Otherwise, let $k \in \bar{N}_{2}$ and notice that either by $N_{\mu}=\emptyset$ (as defined in Action 1), or by Action 1 being repeated in the previous paragraph, the choice of $\bar{N}_{2}$ implies 
$\underline{F}\left(\bar{V}^{1}\right) \leq \bar{v}_{k}^{1}<\bar{F}\left(\bar{V}^{1}\right)$. Define $\bar{V}_{k}^{2} \in \mathcal{R}$ such that $\bar{v}_{k}^{2}=v_{i}^{\prime}$ and $w_{\bar{V}_{k}^{2}}\left(F\left(\bar{V}^{1}\right)\right)=\underline{F}\left(\bar{V}^{1}\right)$, and let profile $\bar{V}^{2}=\left(\bar{V}_{-k}^{1}, \bar{V}_{k}^{2}\right)$. By the process described above for agent $j$, $\bar{F}\left(\bar{V}^{1}\right) \leq \bar{F}\left(\bar{V}^{2}\right)$ and $\underline{F}\left(\bar{V}^{1}\right) \leq \underline{F}\left(\bar{V}^{2}\right)$. Moreover, if $\underline{F}\left(\bar{V}^{1}\right)<\underline{F}\left(\bar{V}^{2}\right)$, perhaps Action 1 needs to be repeated as explained in the previous paragraph. In this case, $\bar{V}^{2}$ is the resulting profile after repeating Action 1, otherwise, $\bar{V}^{2}$ remains unchanged. In both cases, $v_{i}^{\prime}<\bar{F}\left(\bar{V}^{2}\right)$.

Finally, repeat this process for a finite integer $\mu$ (where $\mu$ is smaller than the number of agents, $\mu \leq n-1)$ until the following holds. Set $\bar{N}_{\mu} \subsetneq N$, constructed similarly to $\bar{N}_{1}$ and $\bar{N}_{2}$, is empty. Notice that $v_{i}^{\prime}<\bar{F}\left(\bar{V}^{\mu-1}\right)$ and proceed to Action 3 .

Action 3. Let profile $\hat{V}^{0}=\bar{V}^{\mu-1}$ and recall that $v_{i}^{\prime}<\bar{F}\left(\hat{V}^{0}\right)$. Let $\hat{N} \subsetneq$ $N$ be such that $j \in \hat{N}$ if and only if $\hat{v}_{j}^{0} \neq v_{i}^{\prime}$ and $w_{\hat{V}_{j}^{0}}\left(F\left(\bar{V}^{0}\right)\right)=\underline{F}\left(\hat{V}^{0}\right)$. Let $j \in \hat{N}$ and notice that by $\hat{v}_{j}^{0}<v_{i}^{\prime}<\bar{F}\left(\hat{V}^{0}\right)$ and single-peakedness, $\underline{F}\left(\hat{V}^{0}\right)<$ $\hat{v}_{j}^{0}<\bar{F}\left(\hat{V}^{0}\right)$. Define $\hat{V}_{j}^{1} \in \mathcal{R}$ such that $\hat{v}_{j}^{1}=v_{i}^{\prime}$ and $w_{\hat{V}_{j}^{1}}\left(F\left(\hat{V}^{0}\right)\right)=\bar{F}\left(\hat{V}^{0}\right)$, and let profile $\hat{V}^{1}=\left(\hat{V}_{-j}^{0}, \hat{V}_{j}^{1}\right)$. Assume that $R_{j}=\hat{V}_{j}^{0}$. By strategy-proofness, $w_{R_{j}}\left(F\left(\hat{V}^{0}\right)\right) R_{j} w_{R_{j}}\left(F\left(\hat{V}^{1}\right)\right)$; hence, by single-peakedness, either $\left[\underline{F}\left(\hat{V}^{0}\right) \geq \underline{F}\left(\hat{V}^{1}\right)\right]$ or $\left[\underline{F}\left(\hat{V}^{0}\right)<\underline{F}\left(\hat{V}^{1}\right)\right.$ and $\left.\bar{F}\left(\hat{V}^{0}\right)<\bar{F}\left(\hat{V}^{1}\right)\right]$.

Next, assume that $R_{j}=\hat{V}_{j}^{1}$. If $\bar{F}\left(\hat{V}^{1}\right)>\bar{F}\left(\hat{V}^{0}\right)>\hat{v}_{j}^{1}$, then singlepeakedness implies $w_{R_{j}}\left(F\left(\hat{V}^{0}\right)\right) P_{j} w_{R_{j}}\left(F\left(\hat{V}^{1}\right)\right)$. This violates strategy-proofness since if at profile $\hat{V}^{1}$ agent $j$ deviates by announcing $\hat{V}_{j}^{0}$, his worst point improves. In addition, if $\bar{F}\left(\hat{V}^{1}\right)<\hat{v}_{j}^{1}=b_{R_{j}}\left(F\left(\hat{V}^{0}\right)\right)$, then single-peakedness implies $b_{R_{j}}\left(F\left(\hat{V}^{0}\right)\right) P_{j} b_{R_{j}}\left(F\left(\hat{V}^{1}\right)\right)$. This violates strategy-proofness since if at profile $\hat{V}^{1}$ agent $j$ deviates by announcing $\hat{V}_{j}^{0}$, his best point improves. Therefore, $\underline{F}\left(\hat{V}^{0}\right) \geq \underline{F}\left(\hat{V}^{1}\right)$ and in addition, $v_{i}^{\prime} \leq \bar{F}\left(\hat{V}^{1}\right) \leq \bar{F}\left(\hat{V}^{0}\right)$. Hence, $v_{i}^{\prime}>\underline{F}\left(\hat{V}^{1}\right)$.

Finally, notice that by single-peakedness, $\underline{F}\left(\hat{V}^{0}\right) \geq \underline{F}\left(\hat{V}^{1}\right)$ and $v_{i}^{\prime} \leq \bar{F}\left(\hat{V}^{1}\right) \leq$ $\bar{F}\left(\hat{V}^{0}\right)$ the following holds; for each agent $k \in \hat{N}, w_{\hat{V}_{k}^{0}}\left(F\left(\bar{V}^{0}\right)\right)=\underline{F}\left(\hat{V}^{0}\right)$ implies $w_{\hat{V}_{k}^{1}}\left(F\left(\bar{V}^{1}\right)\right)=\underline{F}\left(\hat{V}^{1}\right)$. Hence, by the process described above for agent $j$, the announced peaks of all agents $k \in \hat{N}$, such that $k \neq j$, can be sequentially changed to $v_{i}^{\prime}$ and profile $\hat{V}^{|\hat{N}|}$ can be constructed. Therefore, since $\hat{v}^{|\hat{N}|}=\left(v_{i}^{\prime}, \ldots, v_{i}^{\prime}\right)$, $\hat{V}^{|\hat{N}|}=V^{\prime}$, and hence, $v_{i}^{\prime}>\underline{F}\left(V^{\prime}\right)$ implies unanimity is violated.

Step 3. Let $V \in \mathcal{R}^{N}$. Without loss of generality, index the agents in $N$ such that $v_{1} \leq \cdots \leq v_{n}$. Let $V^{\prime} \in \mathcal{R}^{N}$ be such that $V^{\prime}=\left(V_{1}, \ldots, V_{1}\right)$. By unanimity, $F\left(V^{\prime}\right)=v_{1}$, hence by Proposition 3.1, $F\left(V^{\prime}\right) \in \mathrm{E}\left(V^{\prime}\right)$. Next, consider profile $V^{2}=\left(V_{-2}^{\prime}, V_{2}\right)$ where $v_{2} \geq v_{2}^{\prime}=v_{1}, \underline{v}^{2}=\underline{v}^{\prime}$, and $\bar{v}^{2} \geq \bar{v}^{\prime}$. Step 1 or Step 2, and $F\left(V^{\prime}\right) \in \mathrm{E}\left(V^{\prime}\right)$ imply $F\left(V^{2}\right) \in \mathrm{E}\left(V^{2}\right)$. Finally, for each $k=\{3, \ldots, n\}$, in increasing order, consider profile $V^{k}=\left(V_{-k}^{k-1}, V_{k}\right)$. By the arguments presented for $V^{2}, F\left(V^{k}\right) \in \mathrm{E}\left(V^{k}\right)$. Therefore, since $V^{n}=V, F(V) \in \mathrm{E}(V)$. 


\section{C Proofs of Propositions 3.4, 3.5, and 3.6}

Before proceeding to the proof of Proposition 3.4 we present a lemma that holds only in domain $\mathcal{R}^{32}$ and concerns strategy-proof correspondences satisfying peaksonliness. Loosely speaking, following an agent's announcement change, there are restrictions on the chosen set.

Lemma 3.7. For each $F \in \mathcal{F}$ satisfying strategy-proofness and peaks-onliness, each $i \in N$, and each pair $V, V^{\prime} \in \mathcal{R}^{N}$ such that $V_{-i}=V_{-i}^{\prime}$, the following hold.

(i) If $v_{i}<\bar{F}(V)$, then $\bar{F}(V) \leq \bar{F}\left(V^{\prime}\right)$, and if in addition $v_{i}<\underline{F}(V)$, then $\underline{F}(V) \leq \underline{F}\left(V^{\prime}\right)$.

(ii) If $v_{i}>\underline{F}(V)$, then $\underline{F}(V) \geq \underline{F}\left(V^{\prime}\right)$, and if in addition $v_{i}>\bar{F}(V)$, then $\bar{F}(V) \geq \bar{F}\left(V^{\prime}\right)$.

Proof. We prove statement (i), the proof of statement (ii) is symmetric. Let $F \in \mathcal{F}$ satisfy strategy-proofness and peaks-onliness. Let pair $V, V^{\prime} \in \mathcal{R}^{N}$ and $i \in N$ be such that $V_{-i}=V_{-i}^{\prime}$ and $v_{i}<\bar{F}(V)$.

Suppose $R_{i}$ is such that $p_{i}=v_{i}$ and $0 P_{i} \bar{F}(V)$. By peaks-onliness, $F\left(V_{-i}, R_{i}\right)=$ $F(V)$. Hence, by single-peakedness and the choice of $R_{i}, w_{R_{i}}\left(F\left(V_{-i}, R_{i}\right)\right)=\bar{F}(V)$. Thus, since $V^{\prime}=\left(V_{-i}, V_{i}^{\prime}\right)$, strategy-proofness implies $\bar{F}(V) R_{i} w_{R_{i}}\left(F\left(V^{\prime}\right)\right)$. Therefore, single-peakedness, the choice of $R_{i}$, and $0 \leq v_{i}<\bar{F}(V)$ imply $w_{R_{i}}\left(F\left(V^{\prime}\right)\right) \geq$ $\bar{F}(V)$, and hence, $\bar{F}\left(V^{\prime}\right) \geq \bar{F}(V)$.

If in addition $v_{i}<\underline{F}(V)$, then by single-peakedness, $b_{R_{i}}\left(F\left(V_{-i}, R_{i}\right)\right)=\underline{F}(V)$. Thus, since $V^{\prime}=\left(V_{-i}, V_{i}^{\prime}\right)$, strategy-proofness implies $\underline{F}(V) R_{i} b_{R_{i}}\left(F\left(V^{\prime}\right)\right)$. Therefore, single-peakedness, $\bar{F}\left(V^{\prime}\right) \geq \bar{F}(V)$, and $v_{i}<\underline{F}(V)$ imply $\underline{F}\left(V^{\prime}\right) \geq \underline{F}(V)$.

The proof of Proposition 3.4 follows and holds only in domain $\mathcal{R}$ because it makes use of Lemma 3.7.

Proof of Proposition 3.4. The proof is split in two parts.

Part 1: We show that strategy-proofness and peaks-onliness imply uncompromisingness.

Let $F \in \mathcal{F}$ satisfy strategy-proofness and peaks-onliness. Let pair $V, V^{\prime} \in \mathcal{R}^{N}$ and $i \in N$ be such that $V_{-i}=V_{-i}^{\prime}$. If $v_{i}=v_{i}^{\prime}$, then peaks-onliness implies uncompromisingness. Hence, let $v_{i} \neq v_{i}^{\prime}$, and by symmetry of arguments, let $v_{i}<v_{i}^{\prime}$. There are four cases. Notice that Case 1.1 overlaps with Case 2.1, while Case 1.2 overlaps with Cases 2.1 and 2.2.

\footnotetext{
${ }^{32}$ It does not hold in domain $\mathcal{S}$ because the proof makes use of non-symmetrical single-peaked preferences.
} 
Case 1.1. Let $v_{i}<v_{i}^{\prime} \leq \underline{F}(V)$. By Lemma 3.7(i), $\underline{F}(V) \leq \underline{F}\left(V^{\prime}\right)$. Moreover, assuming $\underline{F}(V)<\underline{F}\left(V^{\prime}\right)$ results in a contradiction as follows. Since $v_{i}^{\prime}<\underline{F}\left(V^{\prime}\right)$, Lemma 3.7(i) implies $\underline{F}\left(V^{\prime}\right) \leq \underline{F}(V)$. Therefore, $\underline{F}(V)=\underline{F}\left(V^{\prime}\right)$.

Case 1.2. Let $\underline{F}(V)<v_{i}<v_{i}^{\prime}$. By Lemma 3.7(ii), $\underline{F}\left(V^{\prime}\right) \leq \underline{F}(V)$. Hence, $\underline{F}\left(V^{\prime}\right)<v_{i}^{\prime}$, and by Lemma 3.7(ii), $\underline{F}(V) \leq \underline{F}\left(V^{\prime}\right)$. Therefore, $\underline{F}(V)=\underline{F}\left(V^{\prime}\right)$.

Case 2.1. Let $v_{i}<v_{i}^{\prime} \leq \bar{F}(V)$. By Lemma 3.7(i), $\bar{F}(V) \leq \bar{F}\left(V^{\prime}\right)$. Moreover, assuming $\bar{F}(V)<\bar{F}\left(V^{\prime}\right)$ results in a contradiction as follows. Since $v_{i}^{\prime}<\bar{F}\left(V^{\prime}\right)$, Lemma 3.7(i) implies $\bar{F}\left(V^{\prime}\right) \leq \bar{F}(V)<\bar{F}\left(V^{\prime}\right)$. Therefore, $\bar{F}(V)=\bar{F}\left(V^{\prime}\right)$.

Case 2.2. Let $\bar{F}(V)<v_{i}<v_{i}^{\prime}$. By Lemma 3.7(ii), $\bar{F}\left(V^{\prime}\right) \leq \bar{F}(V)$. Hence, $\bar{F}\left(V^{\prime}\right)<v_{i}^{\prime}$, and by Lemma 3.7(ii), $\bar{F}(V) \leq \bar{F}\left(V^{\prime}\right)$. Therefore, $\bar{F}(V)=\bar{F}\left(V^{\prime}\right)$.

Part 2: We show that uncompromisingness implies strategy-proofness and peaks-onliness. Notice that this part of the proof also hold in domain $\mathcal{S}$.

Let $F \in \mathcal{F}$ satisfy uncompromisingness. Let $i \in N$ and pair $V, V^{\prime} \in \mathcal{R}^{N}$ be such that $V_{-i}=V_{-i}^{\prime}$ and $V_{i} \neq V_{i}^{\prime}$. We proceed in two steps.

Step 1. We show that $F$ satisfies peaks-onliness.

Let $v_{i}=v_{i}^{\prime}$. If $v_{i}=\underline{F}(V)$, then assuming $\underline{F}\left(V^{\prime}\right) \neq \underline{F}(V)$ results in a contradiction, since $v_{i}^{\prime}=v_{i} \neq \underline{F}\left(V^{\prime}\right)$ and uncompromisingness imply $\underline{F}(V)=\underline{F}\left(V^{\prime}\right)$. Similarly, if $v_{i}=\bar{F}(V)$, then assuming $\bar{F}\left(V^{\prime}\right) \neq \bar{F}\left(V^{\prime}\right)$ results in a contradiction, since $v_{i}^{\prime}=v_{i} \neq \bar{F}\left(V^{\prime}\right)$ and uncompromisingness imply $\bar{F}(V)=\bar{F}\left(V^{\prime}\right)$. Finally, if $v_{i} \neq \underline{F}(V)$ and $v_{i} \neq \bar{F}(V)$, then by uncompromisingness, $F(V)=F\left(V^{\prime}\right)$. Therefore, $\mathrm{F}$ satisfies peaks-onliness.

Step 2. We show that $F$ satisfies strategy-proofness. Recall that $V_{i} \neq V_{i}^{\prime}$ and by symmetry of arguments, let $v_{i} \leq v_{i}^{\prime}$. By Step $1, F$ satisfies peaks-onliness, hence, if $v_{i}=v_{i}^{\prime}$, then strategy-proofness is satisfied. By symmetry of arguments, let $v_{i}<v_{i}^{\prime}$. We proceed in two stages.

Stage 1. We show that $\underline{F}(V) \leq \underline{F}\left(V^{\prime}\right)$ and $\bar{F}(V) \leq \bar{F}\left(V^{\prime}\right)$. There are 3 cases.

Case 1. Let $v_{i}<\underline{F}(V)$. If $v_{i}<v_{i}^{\prime} \leq \underline{F}(V)$, then by uncompromisingness, $\underline{F}(V)=\underline{F}\left(V^{\prime}\right)$. Otherwise, if $\underline{F}(V)<v_{i}^{\prime}$, then consider the following. Assuming $\underline{F}\left(V^{\prime}\right)<\underline{F}(V)$ results in a contradiction as follows. Let $V_{i}^{1}$ be such that $v_{i}^{1}=$ $\underline{F}\left(V^{\prime}\right)$. Since $v_{i}<\underline{F}(V)$ and $v_{i}^{1}<\underline{F}(V)$, by uncompromisingness, $\underline{F}\left(V_{-i}, V_{i}^{1}\right)=$ $\underline{F}(V)$. However, since $\underline{F}\left(V^{\prime}\right)<v_{i}^{\prime}$ and $v_{i}^{1}=\underline{F}\left(V^{\prime}\right)$, by uncompromisingness, $\underline{F}\left(V_{-i}^{\prime}, V_{i}^{1}\right)=\underline{F}\left(V^{\prime}\right)$. Hence, $\underline{F}(V) \leq \underline{F}\left(V^{\prime}\right)$.

Case 2. Let $v_{i}=\underline{F}(V)<v_{i}^{\prime}$. Assuming $\underline{F}\left(V^{\prime}\right)<\underline{F}(V)$ results in a contradiction as follows. Since $\underline{F}\left(V^{\prime}\right)<v_{i}<v_{i}^{\prime}$, by uncompromisingness, $\underline{F}\left(V^{\prime}\right)=\underline{F}(V)$. Hence, $\underline{F}(V) \leq \underline{F}\left(V^{\prime}\right)$.

Case 3. Let $v_{i}>\underline{F}(V)$. Since $v_{i}<v_{i}^{\prime}$, by uncompromisingness, $\underline{F}(V)=\underline{F}\left(V^{\prime}\right)$. Stage 2. By Stage $1, \underline{F}(V) \leq \underline{F}\left(V^{\prime}\right)$ and $\bar{F}(V) \leq \bar{F}\left(V^{\prime}\right)$. We show that $F$ 
satisfies strategy-proofness. Let $R_{i}=V_{i}$. There are five cases.

Case 1. Let $p_{i}<\underline{F}(V)$. By peaks-onliness, $b_{R_{i}}(F(V))=\underline{F}(V), w_{R_{i}}(F(V))=$ $\bar{F}(V), b_{R_{i}}\left(F\left(V^{\prime}\right)\right)=\underline{F}\left(V^{\prime}\right)$, and $w_{R_{i}}\left(F\left(V^{\prime}\right)\right)=\bar{F}\left(V^{\prime}\right)$. Hence, by singlepeakedness, $i$ 's best and worst points do not improve by deviating at $V$. Therefore, $F(V) R_{i} F\left(V^{\prime}\right)$.

Case 2. Let $p_{i}=\underline{F}(V)$. By peaks-onliness, $b_{R_{i}}(F(V))=p_{i}$ and $w_{R_{i}}(F(V))=$ $\bar{F}(V)$, implying $i$ can't improve on his best point. Regarding his worst point, since $\bar{F}(V) \leq \bar{F}\left(V^{\prime}\right)$, by peaks-onliness, $w_{R_{i}}(F(V)) R_{i} \bar{F}\left(V^{\prime}\right)$. Therefore, $F(V) R_{i} F\left(V^{\prime}\right)$.

Case 3. Let $\underline{F}(V)<p_{i}<\bar{F}(V)$. By peaks-onliness, $b_{R_{i}}(F(V))=p_{i}$ and $w_{R_{i}}(F(V)) \subseteq\{\underline{F}(V), \bar{F}(V)\}$, implying agent $i$ can't improve on his best point. Regarding his worst point(s), since $\underline{F}(V)<p_{i} \leq v_{i}^{\prime}$, by uncompromisingness, $\underline{F}(V)=\underline{F}\left(V^{\prime}\right)$. Since also $\bar{F}(V) \leq \bar{F}\left(V^{\prime}\right)$, by peaks-onliness, $w_{R_{i}}(F(V)) R_{i} w_{R_{i}}\left(F\left(V^{\prime}\right)\right)$. Therefore, $F(V) R_{i} F\left(V^{\prime}\right)$.

Case 4. Let $p_{i}=\bar{F}(V)$. By peaks-onliness, $b_{R_{i}}(F(V))=p_{i}$ and $w_{R_{i}}(F(V))=$ $\underline{F}(V)$, implying $i$ can't improve on his best point. Regarding his worst point, if $\underline{F}(V)<\bar{F}(V)$, then since $\underline{F}(V)<p_{i} \leq v_{i}^{\prime}$, and by uncompromisingness, $\underline{F}(V)=$ $\underline{F}\left(V^{\prime}\right)$. Hence, $\bar{F}(V) \leq \bar{F}\left(V^{\prime}\right)$ implies $w_{R_{i}}(F(V)) R_{i} w_{R_{i}}\left(F\left(V^{\prime}\right)\right)$. Otherwise, if $\underline{F}(V)=\bar{F}(V)$, then $w_{R_{i}}(F(V))=p_{i}$, implying $i$ can't improve on his worst point. Therefore, in both cases $F(V) R_{i} F\left(V^{\prime}\right)$.

Case 5. Let $p_{i}>\bar{F}(V)$. Since $\bar{F}(V)<p_{i} \leq v_{i}^{\prime}$, by uncompromisingness, $F(V)=F\left(V^{\prime}\right)$. Therefore, $F(V) I_{i} F\left(V^{\prime}\right)$.

Before proceeding to the proof of Proposition 3.5 we present two lemmata that hold only in domain $\mathcal{S}$ (because they make use of Proposition 3.2) and concern strategy-proof correspondences satisfying voter-sovereignty. Loosely speaking, both show cases where following a change in the preferences of some agents, there are restrictions in the minimum and maximum chosen alternatives.

Lemma 3.8. For each $F \in \mathcal{F}$ satisfying strategy-proofness and voter-sovereignty, each $V \in \mathcal{S}^{N}$, and each $x \in A$ the following hold.

(i) Let $V^{\prime} \in \mathcal{S}^{N}$ be as follows. For each each $i \in N$, if $v_{i} \leq x$, then $v_{i}^{\prime}=x$, otherwise $v_{i}^{\prime}=v_{i}$. Then, $x \leq \bar{F}(V)$ implies $\bar{F}(V) \leq \bar{F}\left(V^{\prime}\right)$; in addition, $x \leq \underline{F}(V)$ implies $\underline{F}(V) \leq \underline{F}\left(V^{\prime}\right)$.

(ii) Let $V^{\prime} \in \mathcal{S}^{N}$ be as follows. For each $i \in N$, if $v_{i} \geq x$, then $v_{i}^{\prime}=x$, otherwise $v_{i}^{\prime}=v_{i}$. Then, $x \geq \underline{F}(V)$ implies $\underline{F}(V) \geq \underline{F}\left(V^{\prime}\right)$; in addition, $x \geq \bar{F}(V)$ implies $\bar{F}(V) \geq \bar{F}\left(V^{\prime}\right)$.

Proof. We prove statement (i), the proof of statement (ii) is symmetric. Let $F \in \mathcal{F}$ satisfy strategy-proofness and voter-sovereignty. By Proposition 3.2, $F$ also 
satisfies efficiency. Let $V \in \mathcal{S}^{N}$ and $x \in A$ be such that $x \leq \bar{F}(V)$; and without loss of generality, let $v_{1} \leq \cdots \leq v_{n}$. Moreover, let $V^{\prime} \in \mathcal{S}^{N}$ be defined as follows. For each $i \in N$, if $v_{i} \leq x$, then $v_{i}^{\prime}=x$, otherwise $v_{i}^{\prime}=v_{i}$. Let $M=\{1, \ldots, m\} \subseteq N$ be such that $i \in M$ implies $v_{i}^{\prime}=x$. Hence, $V^{\prime}=\left(V_{x}, \ldots, V_{x}, V_{m+1}, \ldots, V_{n}\right)$, where $V_{x} \in \mathcal{S}$ and $v_{x}=x$.

Begin from profile $V$. By efficiency and Proposition 3.1, $F(V) \subseteq \operatorname{Conv}(V)$. Let $R_{1}=V_{1}$, hence $v_{1} \leq x \leq \bar{F}(V)$ implies $w_{R_{1}}(F(V))=\bar{F}(V)$. Next, let $V_{1}^{1}=V_{x}$ and consider profile $V^{1}=\left(V_{-1}, V_{1}^{1}\right)$. By efficiency and Proposition 3.1, $F\left(V^{1}\right) \subseteq$ $\operatorname{Conv}\left(V^{1}\right)$, and by strategy-proofness, $w_{R_{1}}(F(V)) R_{1} w_{R_{1}}\left(F\left(V^{1}\right)\right)$. Therefore, by single-peakedness, $v_{1} \leq \bar{F}(V) \leq \bar{F}\left(V^{1}\right)$. If $V^{1}=V^{\prime}$, then we are done. Otherwise, for each $k \in\{2, \ldots, m\}$, in increasing order, consider profile $V^{k}=\left(V_{-k}^{k-1}, V_{k}^{k}\right)$. By the arguments presented for $V^{1}, \bar{F}\left(V^{k-1}\right) \leq \bar{F}\left(V^{k}\right)$. Therefore, $V^{m}=V^{\prime}$ implies $\bar{F}(V) \leq \bar{F}\left(V^{\prime}\right)$.

If in addition $x \leq \underline{F}(V)$, then $b_{R_{1}}(F(V))=\underline{F}(V)$. In this case, begin from profile $V$ and construct profile $V^{\prime}$ as shown above. By the same arguments to the ones presented above, but expressed for the best alternative instead of the worst, it follows that $\underline{F}(V) \leq \underline{F}\left(V^{\prime}\right)$.

Lemma 3.9. For each $F \in \mathcal{F}$ satisfying strategy-proofness and voter-sovereignty, and each $V \in \mathcal{S}^{N}$ the following hold.

(i) Let $M \subseteq N$ be such that $i \in M$ implies $v_{i}=\underline{v}$. Let $V^{\prime} \in \mathcal{S}^{N}$ be as follows. For each $i \in N$, if $i \in M$, then $v_{i}^{\prime} \leq v_{i}$, otherwise $v_{i}^{\prime}=v_{i}$. Then, $\underline{v}<\bar{F}(V)$ implies $\bar{F}(V) \leq \bar{F}\left(V^{\prime}\right)$; in addition, $\underline{v}<\underline{F}(V)$ implies $\underline{F}(V) \leq \underline{F}\left(V^{\prime}\right)$.

(ii) Let $M \subseteq N$ be such that $i \in M$ implies $v_{i}=\bar{v}$. Let $V^{\prime} \in \mathcal{S}^{N}$ be as follows. For each $i \in N$, if $i \in M$, then $v_{i}^{\prime} \geq v_{i}$, otherwise $v_{i}^{\prime}=v_{i}$. Then, $\bar{v}>\underline{F}(V)$ implies $\underline{F}(V) \geq \underline{F}\left(V^{\prime}\right)$; in addition, $\bar{v}>\bar{F}(V)$ implies $\bar{F}(V) \geq \bar{F}\left(V^{\prime}\right)$.

Proof. We prove statement (i), the proof of statement (ii) is symmetric. Let $F \in \mathcal{F}$ satisfy strategy-proofness and voter-sovereignty, and $V \in \mathcal{S}^{N}$ be such that $\underline{v}<\bar{F}(V)$. By Proposition 3.2, $F$ also satisfies efficiency; hence, Proposition 3.1 implies $\underline{v}<\bar{F}(V) \leq \bar{v}$. In addition, let $M \subseteq N$ be such that $i \in M$ implies $v_{i}=\underline{v}$, and without loss of generality, let $M=(1, \ldots, m)$; hence, $\underline{v}<\bar{v}$ implies $M \subsetneq N$. Moreover, let $V^{\prime} \in \mathcal{S}^{N}$ be as follows. For each $i \in N$, if $i \in M$, then $v_{i}^{\prime} \leq v_{i}$, otherwise $v_{i}^{\prime}=v_{i}$. Finally, without loss of generality, let $v_{1}^{\prime} \leq \cdots \leq v_{m}^{\prime}<v_{m+1}^{\prime} \leq$ $\cdots \leq v_{n}^{\prime}$.

Begin from profile $V$ and let $\delta=|\underline{v}-\bar{F}(V)|>0$. Assume $R_{1}=V_{1}$. By single-peakedness, $w_{R_{1}}(F(V))=\bar{F}(V)$. Change the announced preferences of agent 1 to $V_{1}^{1} \in \mathcal{S}$ as follows. If $\left|v_{m}^{\prime}-\underline{v}\right|<\delta$, then set $v_{1}^{1}=v_{m}^{\prime}$, otherwise, set 
$v_{1}^{1}=\underline{v}-\frac{\delta}{2}$. By efficiency and Proposition 3.1, $F\left(V_{-1}, V_{1}^{1}\right) \subseteq \operatorname{Conv}\left(V_{-1}, V_{1}^{1}\right)$. By strategy-proofness, $w_{R_{1}}(F(V)) R_{1} w_{R_{1}}\left(F\left(V_{-1}, V_{1}^{1}\right)\right)$. Therefore, $\left|v_{1}^{1}-\underline{v}\right|<\delta$ implies (in domain $\mathcal{S}$ ) that $\bar{F}(V) \leq \bar{F}\left(V_{-1}, V_{1}^{1}\right)$. Following this, sequentially repeat this process for all agents $i \in\{2, \ldots, m\}$ (if such agents exist) and construct profile $V^{1}=\left(V_{1}^{1}, \ldots, V_{m}^{1}, V_{m+1}, \ldots, V_{n}\right)$, where $\bar{F}(V) \leq \bar{F}\left(V^{1}\right)$. If $v_{1}^{1}=v_{m}^{\prime}$, proceed to the next paragraph. Otherwise, let $\delta^{1}=\left|\underline{v}^{1}-\bar{F}\left(V^{1}\right)\right|>0$, assume $R_{1}=V_{1}^{1}$, and repeat the process to construct profile $V^{2}$, where $\bar{F}(V) \leq \bar{F}\left(V^{2}\right)$. If $v_{1}^{2}=v_{m}^{\prime}$, proceed to the next paragraph. Otherwise, keep repeating this process until the profile $\bar{V}^{m}=\left(V_{m}^{\prime}, \ldots, V_{m}^{\prime}, V_{m+1}, \ldots, V_{n}\right)$ has been constructed, where $\bar{F}(V) \leq \bar{F}\left(\bar{V}^{m}\right)$.

Next, repeat the process described above for all agents $i \in$ $\{1, \ldots, m-1\}$ (if such agents exist) and construct profile $\bar{V}^{m-1}=$ $\left(V_{m-1}^{\prime}, \ldots, V_{m-1}^{\prime}, V_{m}^{\prime}, V_{m+1}, \ldots, V_{n}\right)$, where $\bar{F}(V) \leq \bar{F}\left(\bar{V}^{m-1}\right)$.

Finally, continue repeating this whole process until the profile $\bar{V}^{1}=V^{\prime}=$ $\left(V_{1}^{\prime}, \ldots, V_{m}^{\prime}, V_{m+1}, \ldots, V_{n}\right)$ has been constructed, where $\bar{F}(V) \leq \bar{F}\left(V^{\prime}\right)$.

If in addition, $\underline{v}<\underline{F}(V)$, begin from profile $V$, let $\delta=|\underline{v}-\underline{F}(V)|>0$, and construct profile $V^{\prime}$ as shown above. By the same arguments to the ones presented above, but expressed for the best alternative instead of the worst, it follows that $\underline{F}(V) \leq \underline{F}\left(V^{\prime}\right)$.

We now proceed with the proof of Proposition 3.5 that holds only in domain $\mathcal{S}$ because it makes indirect use of Proposition 3.2 through Lemmas 3.8 and 3.9.

Proof of Proposition 3.5. Let $F \in \mathcal{F}$. Part 2 of Proposition 3.4 on page 96 (which also holds in $\mathcal{S}$ ) shows that if $F$ satisfies uncompromisingness then it also satisfies strategy-proofness. Hence, it follows that statement (ii) implies statement (i). Next, we show that statement (i) implies statement (ii).

Let $F$ satisfy strategy-proofness and voter-sovereignty. Let $i \in N$ and pair $V, V^{\prime} \in \mathcal{S}^{N}$ be such that $V_{-i}=V_{-i}^{\prime}$. Without loss of generality, assume $v_{1} \leq \cdots \leq$ $v_{n}$. Since $v_{i}=v_{i}^{\prime}$ trivially satisfies uncompromisingness in domain $\mathcal{S}$, let $v_{i} \neq v_{i}^{\prime}$. There are six cases.

Case 1.1. Let $v_{i}^{\prime}<v_{i} \leq \underline{F}(V)$. Since $v_{i}=\underline{F}(V)=\bar{F}(V)$ trivially satisfies uncompromisingness, let $v_{i}<\bar{F}(V)$. In addition, let $M \subsetneq N$ be such that $j \in M$ if and only if $v_{j} \leq v_{i}$. Begin from profile $V$ and consider profile $V^{1}$ to be such that $V_{-M}=V_{-M}^{1}$ and where each agent $j \in M$ announces preferences $V_{j}^{1}=V_{i}$. By construction of $V^{1}$ and Lemma 3.8(i), $\underline{F}\left(V^{1}\right) \geq \underline{F}(V)$ and $\bar{F}\left(V^{1}\right) \geq \bar{F}(V)$. Moreover, begin from profile $V^{1}$ and consider profile $V$. Since for each $j \in M, v_{j} \leq$ $v_{j}^{1}=\underline{v}^{1}$, and for each $k \in N \backslash M, v_{k}=v_{k}^{1}>\underline{v}^{1}$, by Lemma 3.9(i), $\bar{F}(V) \geq \bar{F}\left(V^{1}\right)$ 
and in addition, if $v_{i}<\underline{F}(V)$, then $\underline{F}(V) \geq \underline{F}\left(V^{1}\right)$. Therefore, $\underline{F}\left(V^{1}\right)=\underline{F}(V)$ and $\bar{F}\left(V^{1}\right)=\bar{F}(V)$.

Next, begin from profile $V^{1}$ and consider profile $V^{\prime}$. Since for each $j \in M, v_{j}^{\prime} \leq$ $v_{j}^{1}=\underline{v}^{1}$, and for each $k \in N \backslash M, v_{k}^{\prime}=v_{k}^{1}>\underline{v}^{1}$, by Lemma 3.9(i), $\bar{F}\left(V^{\prime}\right) \geq \bar{F}\left(V^{1}\right)$, and in addition, if $v_{i}<\underline{F}\left(V^{1}\right)=\underline{F}(V)$, then $\underline{F}\left(V^{\prime}\right) \geq \underline{F}\left(V^{1}\right)$. Finally, begin from profile $V^{\prime}$ and consider profile $V^{1}$. Since for each $j \in M, v_{j}^{\prime} \leq v_{j}^{1}=v_{i}^{\prime}$, and for each $k \in N \backslash M, v_{k}^{\prime}=v_{k}^{1}>v_{i}^{\prime}$, by Lemma 3.8(i), $\bar{F}\left(V^{1}\right) \geq \bar{F}\left(V^{\prime}\right)$ and $\underline{F}\left(V^{1}\right) \geq \underline{F}\left(V^{\prime}\right)$. Therefore, $\bar{F}\left(V^{1}\right)=\bar{F}(V)=\bar{F}\left(V^{\prime}\right)$ and $\underline{F}\left(V^{1}\right)=\underline{F}(V)=\underline{F}\left(V^{\prime}\right)$.

Case 1.2. Let $v_{i}^{\prime}>v_{i} \geq \bar{F}(V)$. The proof is symmetric to Case 1.1.

Case 2.1. Let $v_{i} \leq \underline{F}(V)$ and $v_{i}<v_{i}^{\prime}$. If $v_{i}^{\prime}>\bar{F}(V)$, then uncompromisingness is trivially satisfied; hence, let $v_{i}^{\prime} \leq \bar{F}(V)$. In addition, let $M \subsetneq N$ be such that $j \in M$ if and only if $v_{j} \leq v_{i}^{\prime}$. Begin from profile $V$ and consider profile $V^{1}$ to be such that $V_{-M}=V_{-M}^{1}$ and where each agent $j \in M$ announces preferences $V_{j}^{1}=V_{i}^{\prime}$. By construction of $V^{1}$ and Lemma 3.8(i), $\bar{F}\left(V^{1}\right) \geq \bar{F}(V)$ and in addition, if $v_{i}^{\prime} \leq \underline{F}(V)$, then $\underline{F}\left(V^{1}\right) \geq \underline{F}(V)$. Moreover, begin from profile $V^{1}$ and consider profile $V$. Since for each $j \in M, v_{j} \leq v_{j}^{1}=\underline{v}^{1}$, and for each $k \in N \backslash M$, $v_{k}=v_{k}^{1}>\underline{v}^{1}$, by Lemma 3.9(i), if $v_{i}<\bar{F}\left(V^{1}\right)$, then $\bar{F}(V) \geq \bar{F}\left(V^{1}\right)$ and in addition, if $v_{i}<\underline{F}\left(V^{1}\right)$, then $\underline{F}(V) \geq \underline{F}\left(V^{1}\right)$. Therefore, $\bar{F}\left(V^{1}\right)=\bar{F}(V)$ and in addition, if $v_{i}^{\prime} \leq \underline{F}(V)$, then $\underline{F}\left(V^{1}\right)=\underline{F}(V)$. There are three sub-cases.

(i) Let $v_{i}^{\prime}=\bar{F}(V)$. Assume $R_{i}=V_{i}^{\prime}$. Hence, $b_{R_{i}}(F(V))=\bar{F}(V)$. Since $V_{-i}=V_{-i}^{\prime}$, by strategy-proofness, $\bar{F}(V)=v_{i}^{\prime} \in F\left(V^{\prime}\right)$. Thus, $\bar{F}\left(V^{\prime}\right) \geq \bar{F}(V)$. Moreover, begin from profile $V^{\prime}$ and consider profile $V^{1}$. Since for each $j \in M$, $v_{j}^{\prime} \leq v_{j}^{1}=v_{i}^{\prime}$, and for each $k \in N \backslash M, v_{k}^{\prime}=v_{k}^{1}>v_{i}^{\prime}$, by Lemma 3.8(i), $\bar{F}\left(V^{1}\right) \geq$ $\bar{F}\left(V^{\prime}\right)$. Therefore, $\bar{F}\left(V^{1}\right)=\bar{F}(V)=\bar{F}\left(V^{\prime}\right)$.

(ii) Let $v_{i}^{\prime}<\bar{F}(V)$. Begin from profile $V^{1}$ and consider profile $V^{\prime}$. Since for each $j \in M, v_{j}^{\prime} \leq v_{j}^{1}=\underline{v}^{1}$, and for each $k \in N \backslash M, v_{k}^{\prime}=v_{k}^{1}>\underline{v}^{1}$, by $v_{i}^{\prime}<\bar{F}(V)$ and Lemma 3.9(i), $\bar{F}\left(V^{\prime}\right) \geq \bar{F}\left(V^{1}\right)$, and in addition, if $v_{i}^{\prime}<\underline{F}(V)$, then $\underline{F}\left(V^{\prime}\right) \geq \underline{F}\left(V^{1}\right)$. Moreover, begin from profile $V^{\prime}$ and consider profile $V^{1}$. Since for each $j \in M, v_{j}^{\prime} \leq v_{j}^{1}=v_{i}^{\prime}$, and for each $k \in N \backslash M, v_{k}^{\prime}=v_{k}^{1}>v_{i}^{\prime}$, by $v_{i}^{\prime}<\bar{F}(V)$ and Lemma 3.8(i), $\bar{F}\left(V^{1}\right) \geq \bar{F}\left(V^{\prime}\right)$, and in addition, if $v_{i}^{\prime}<\underline{F}\left(V^{\prime}\right)$, then $\underline{F}\left(V^{1}\right) \geq \underline{F}\left(V^{\prime}\right)$. Therefore, $\bar{F}\left(V^{1}\right)=\bar{F}\left(V^{\prime}\right)=\bar{F}(V)$ and in addition, if $v_{i}^{\prime}<\underline{F}(V)$, then $\underline{F}\left(V^{1}\right)=\underline{F}\left(V^{\prime}\right)=\underline{F}(V)$.

(iii) Let $v_{i}^{\prime}=\underline{F}(V)$. If $v_{i}^{\prime}=\underline{F}(V)=\bar{F}(V)$, then uncompromisingness is trivially satisfied; hence, let $v_{i}^{\prime}=\underline{F}(V)<\bar{F}(V)$. As shown in the previous subcase, $\bar{F}\left(V^{\prime}\right)=\bar{F}(V)$. Assume $R_{i}=V_{i}$. Since $v_{i}<v_{i}^{\prime}$, by single-peakedness, $b_{R_{i}}(F(V))=\underline{F}(V)$ and $w_{R_{i}}(F(V))=\bar{F}(V)$. Hence, $V_{-i}^{\prime}=V_{-i}$ and strategyproofness imply $b_{R_{i}}(F(V)) R_{i} b_{R_{i}}\left(F\left(V^{\prime}\right)\right)$. Thus, by $\bar{F}\left(V^{\prime}\right)=\bar{F}(V)$ and single- 
peakedness, $b_{R_{i}}(F(V))=\underline{F}(V) \leq \underline{F}\left(V^{\prime}\right)$. Finally, assume $R_{i}=V_{i}^{\prime}$. Since $v_{i}^{\prime} \leq$ $\underline{F}\left(V^{\prime}\right) \leq \bar{F}\left(V^{\prime}\right)$, by single-peakedness, $b_{R_{i}}\left(F\left(V^{\prime}\right)\right)=\underline{F}\left(V^{\prime}\right)$ and $w_{R_{i}}\left(F\left(V^{\prime}\right)\right)=$ $\bar{F}\left(V^{\prime}\right)$. Hence, $V_{-i}^{\prime}=V_{-i}$ and strategy-proofness imply $b_{R_{i}}\left(F\left(V^{\prime}\right)\right) R_{i} b_{R_{i}}(F(V))$. Thus, by $\bar{F}\left(V^{\prime}\right)=\bar{F}(V)$ and single-peakedness, $b_{R_{i}}\left(F\left(V^{\prime}\right)\right)=\underline{F}\left(V^{\prime}\right) \leq \underline{F}(V)$. Therefore, $\underline{F}(V)=\underline{F}\left(V^{\prime}\right)$.

Case 2.2. Let $v_{i} \geq \bar{F}(V)$ and $v_{i}>v_{i}^{\prime}$. The proof is symmetric to Case 2.1.

Case 3.1. Let $\underline{F}(V)<v_{i}<\bar{F}(V)$ and $v_{i}^{\prime}>v_{i}$. In addition, let $M \subsetneq N$ be such that $j \in M$ if and only if $v_{j} \geq v_{i}^{\prime}$. Begin from profile $V$ and consider profile $V^{1}$ to be such that $V_{-M}=V_{-M}^{1}$ and where each agent $j \in M$ announces preferences $V_{j}^{1}=V_{i}^{\prime}$. By construction of $V^{1}$ and Lemma 3.8(ii), $\underline{F}(V) \geq \underline{F}\left(V^{1}\right)$. Moreover, begin from profile $V^{1}$ and consider profile $V$. Since for each $j \in M, v_{j} \geq v_{j}^{1}=\bar{v}^{1}$, and for each $k \in N \backslash M, v_{k}=v_{k}^{1}<\bar{v}^{1}$, by $v_{i}^{\prime}>\underline{F}\left(V^{1}\right)$ and Lemma 3.9(ii), $\underline{F}\left(V^{1}\right) \geq \underline{F}\left(V^{\prime}\right)$. Therefore, $\underline{F}(V)=\underline{F}\left(V^{1}\right)$.

Next, begin from profile $V^{1}$ and consider profile $V^{\prime}$. Since for each $j \in M$, $v_{j}^{\prime} \geq v_{j}^{1}=\bar{v}^{1}$, and for each $k \in N \backslash M, v_{k}^{\prime}=v_{k}^{1}<\bar{v}^{1}$, by $\bar{v}^{1}>\underline{F}\left(V^{1}\right)$ and Lemma 3.9(ii), $\underline{F}\left(V^{\prime}\right) \leq \underline{F}\left(V^{1}\right)$. Finally, begin from profile $V^{\prime}$ and consider profile $V^{1}$. Since for each $j \in M, v_{j}^{\prime} \geq v_{j}^{1}=v_{i}^{\prime}$, and for each $k \in N \backslash M, v_{k}^{\prime}=v_{k}^{1}<v_{i}^{\prime}$, by Lemma 3.8(ii), $\underline{F}\left(V^{1}\right) \leq \underline{F}\left(V^{\prime}\right)$. Therefore, $\underline{F}\left(V^{1}\right)=\underline{F}(V)=\underline{F}\left(V^{\prime}\right)$.

If $v_{i}^{\prime}>\bar{F}(V)$, then we are done. If $v_{i}^{\prime} \leq \bar{F}(V)$, then let $L \subsetneq N$ be such that $j \in L$ if and only if $v_{j} \leq v_{i}^{\prime}$. Begin from profile $V$ and consider profile $V^{2}$ to be such that $V_{-L}=V_{-L}^{2}$ and where each agent $j \in L$ announces preferences $V_{j}^{2}=V_{i}^{\prime}$. By construction of $V^{2}$ and Lemma 3.8(i), $\bar{F}(V) \leq \bar{F}\left(V^{2}\right)$. There are two sub-cases.

(i) Let $v_{i}^{\prime}<\bar{F}(V)$. Begin from profile $V^{2}$ and consider profile $V$. Since for each $j \in L, v_{j}^{\prime} \leq v_{j}^{2}=\underline{v}^{2}$, and for each $k \in N \backslash L, v_{k}^{\prime}=v_{k}^{2}>\underline{v}^{2}$, by $\underline{v}^{2}<\bar{F}\left(V^{2}\right)$ and Lemma 3.9(i), $\bar{F}\left(V^{2}\right) \leq \bar{F}(V)$. Therefore, $\bar{F}(V)=\bar{F}\left(V^{2}\right)$.

Next, begin from profile $V^{\prime}$ and consider profile $V^{2}$. Since for each $j \in L$, $v_{j}^{\prime} \leq v_{j}^{2}=v_{i}^{\prime}$, and for each $k \in N \backslash L, v_{k}^{\prime}=v_{k}^{2}<v_{i}^{\prime}$, by Lemma 3.8(i), $\bar{F}\left(V^{2}\right) \geq$ $\bar{F}\left(V^{\prime}\right)$. Finally, begin from profile $V^{2}$ and consider profile $V^{\prime}$. Since for each $j \in L, v_{j}^{\prime} \leq v_{j}^{2}=\underline{v}^{2}$, and for each $k \in N \backslash L, v_{k}^{\prime}=v_{k}^{2}>\underline{v}^{2}$, by $\underline{v}^{2}<\bar{F}\left(V^{2}\right)$ and Lemma 3.9(i), $\bar{F}\left(V^{\prime}\right) \geq \bar{F}\left(V^{2}\right)$. Therefore, $\bar{F}\left(V^{2}\right)=\bar{F}(V)=\bar{F}\left(V^{\prime}\right)$.

(ii) Let $v_{i}^{\prime}=\bar{F}(V)$. Assume $R_{i}=V_{i}^{\prime}$. By single-peakedness, $b_{R_{i}}(F(V))=v_{i}^{\prime}$. Hence, $V_{-i}=V_{-i}^{\prime}$ and strategy-proofness imply $v_{i}^{\prime} \in F\left(V^{\prime}\right)$. Thus, $\bar{F}\left(V^{\prime}\right) \geq \bar{F}(V)$.

Assuming $\bar{F}\left(V^{\prime}\right)>\bar{F}(V)$ results in a contradiction as follows. Begin from profile $V$ and consider profile $V^{2}$. Since for each $j \in L, v_{j} \leq v_{j}^{2}=v_{i}^{\prime}$, and for each $k \in N \backslash L, v_{k}=v_{k}^{2}>v_{i}^{\prime}$, by Lemma 3.8(i), $\bar{F}(V) \leq \bar{F}\left(V^{2}\right)$. Moreover, begin from profile $V^{2}$ and consider profile $V$. Since for each $j \in L, v_{j} \leq v_{j}^{2}=\underline{v}^{2}$, and for each $k \in N \backslash L, v_{k}=v_{k}^{2}>\underline{v}^{2}$, by $\underline{v}^{2}<\bar{F}\left(V^{2}\right)$ and Lemma 3.9(i), $\bar{F}(V) \geq \bar{F}\left(V^{2}\right)$. 
Therefore, $\bar{F}(V)=\bar{F}\left(V^{2}\right)$.

Next, begin from profile $V^{\prime}$ and consider profile $V^{2}$ as described in the previous sub-case. Since for each $j \in L, v_{j}^{\prime} \leq v_{j}^{2}=v_{i}^{\prime}$, and for each $k \in N \backslash L, v_{k}^{\prime}=v_{k}^{2}>v_{i}^{\prime}$, by Lemma 3.8(i), $\bar{F}\left(V^{\prime}\right) \leq \bar{F}\left(V^{2}\right)$. Finally, begin from profile $V^{2}$ and consider profile $V^{\prime}$. Since for each $j \in L, v_{j}^{\prime} \leq v_{j}^{2}=\underline{v}^{2}$, and for each $k \in N \backslash L, v_{k}^{\prime}=v_{k}^{2}>\underline{v}^{2}$, by $\underline{v}^{2}<\bar{F}\left(V^{2}\right)$ and Lemma 3.9(i), $\bar{F}\left(V^{\prime}\right) \geq \bar{F}\left(V^{2}\right)$. Therefore, $\bar{F}\left(V^{2}\right)=\bar{F}\left(V^{\prime}\right)$. Therefore, $\bar{F}\left(V^{2}\right)=\bar{F}(V)=\bar{F}\left(V^{\prime}\right)$.

Case 3.2. Let $\underline{F}(V)<v_{i}<\bar{F}(V)$ and $v_{i}^{\prime}<v_{i}$. The proof is symmetric to Case 3.1 .

Finally, we present the proof of Proposition 3.6.

Proof of Proposition 3.6. Let $F \in \mathcal{F}$ satisfy strategy-proofness, min/max continuity, and voter-sovereignty. By Proposition 3.3, $F$ also satisfies efficiency. Let pair $V, V^{\prime} \in \mathcal{R}^{N}$ and $i \in N$ be such that $V_{-i}=V_{-i}^{\prime}$. There are five cases.

Case 1.1. Let $v_{i}<\underline{F}(V)$ and $v_{i}^{\prime} \leq \bar{F}(V)$. Notice that if $R_{i}=V_{i}$, by singlepeakedness, $b_{R_{i}}(F(V))=\underline{F}(V)$ and $w_{R_{i}}(F(V))=\bar{F}(V)$.

Assuming $v_{i} \geq \underline{F}\left(V^{\prime}\right)$ leads to a contradiction as follows. By $\min / \max$ continuity, there exists $V_{i}^{*} \in \mathcal{R}$ such that $\underline{F}\left(V_{-i}, V_{i}^{*}\right)=v_{i}$. Assume $R_{i}=V_{i}$. By single-peakedness, $b_{R_{i}}\left(F\left(V_{-i}, V_{i}^{*}\right)\right)=v_{i} \notin F(V)$. Hence, if at profile $V$ agent $i$ deviates by announcing $V_{i}^{*}$, his best point improves. This contradicts strategyproofness. Therefore, $v_{i}<\underline{F}\left(V^{\prime}\right) \leq \bar{F}\left(V^{\prime}\right)$.

Next, assuming $\underline{F}\left(V^{\prime}\right)<\underline{F}(V)$ or $\bar{F}\left(V^{\prime}\right)<\bar{F}(V)$ leads to a contradiction as follows. Assume $R_{i}=V_{i}$. By $v_{i}<\underline{F}\left(V^{\prime}\right) \leq \bar{F}\left(V^{\prime}\right)$ and single-peakedness, $b_{R_{i}}\left(F\left(V^{\prime}\right)\right) P_{i} b_{R_{i}}(F(V))$ or $w_{R_{i}}\left(F\left(V^{\prime}\right)\right) P_{i} b_{R_{i}}(F(V))$. Hence, if at profile $V$ agent $i$ deviates by announcing $V_{i}^{\prime}$, his best point or his worst point improves. This contradicts strategy-proofness. Therefore, $\underline{F}\left(V^{\prime}\right) \geq \underline{F}(V)$ and $\bar{F}\left(V^{\prime}\right) \geq \bar{F}(V)$.

Finally, assuming $\underline{F}(V)<\underline{F}\left(V^{\prime}\right)$ or $\bar{F}(V)<\bar{F}\left(V^{\prime}\right)$ leads to a contradiction as follows. Assume $R_{i}=V_{i}^{\prime}$. By $v_{i}^{\prime} \leq \underline{F}(V) \leq \bar{F}(V)$ and single-peakedness, $b_{R_{i}}(F(V)) P_{i} b_{R_{i}}\left(F\left(V^{\prime}\right)\right)$ or $w_{R_{i}}(F(V)) P_{i} b_{R_{i}}\left(F\left(V^{\prime}\right)\right)$. Hence, if at profile $V^{\prime}$ agent $i$ deviates by announcing $V_{i}$, his best point or his worst point improves. This contradicts strategy-proofness.

Therefore, $\bar{F}\left(V^{\prime}\right)=\bar{F}(V)$ and in addition, if $v_{i}^{\prime} \leq \underline{F}(V)$, then $\underline{F}\left(V^{\prime}\right)=\underline{F}(V)$.

Case 1.2. Let $v_{i}=\underline{F}(V)$ and $v_{i}^{\prime} \leq \bar{F}(V)$. By the same arguments to the ones presented in Case 1.1 for the maximum point chosen, it follows that $\bar{F}\left(V^{\prime}\right)=\bar{F}(V)$.

Case 2.1. Let $v_{i}>\bar{F}(V)$ and $v_{i}^{\prime} \geq \underline{F}(V)$. By symmetric arguments to those presented in Case 1.1, it follows that $\underline{F}\left(V^{\prime}\right)=\underline{F}(V)$ and in addition, if $v_{i}^{\prime} \geq \bar{F}(V)$, then $\bar{F}\left(V^{\prime}\right)=\bar{F}(V)$. 
Case 2.2. Let $v_{i}=\bar{F}(V)$ and $v_{i}^{\prime} \geq \underline{F}(V)$. By symmetric arguments to those presented in Case 1.2, it follows that $\underline{F}\left(V^{\prime}\right)=\underline{F}(V)$.

Case 3. Let $\underline{F}(V)<v_{i}<\bar{F}(V)$. By symmetry of arguments, let $v_{i} \geq v_{i}^{\prime}$. Without loss of generality, let $v_{1} \leq \cdots \leq v_{n}$ and notice that by efficiency and Proposition 3.1, $F(V) \subseteq \operatorname{Conv}(V)$; hence, agent $i \notin\{1, n\}$. In addition, for each agent $j \in N \backslash\{i\}$, define preferences $\bar{V}_{j} \in \mathcal{R}$ be such that $\bar{V}_{j}=V_{i}^{\prime}$.

Begin from profile $V$ and consider profile $V^{1}=\left(V_{-1}, \bar{V}_{1}\right)$. By efficiency and Proposition 3.1, $F(V) \subseteq \operatorname{Conv}(V)$. Hence, since $v_{1}=\underline{v}$, by either Case 1.1 (if $v_{1}<\underline{F}(V)$ ) or Case 1.2 (if $v_{1}=\underline{F}(V)$ ), $\bar{F}\left(V^{1}\right)=\bar{F}(V)$. Moreover, by efficiency and Proposition 3.1, $F\left(V^{1}\right) \subseteq \operatorname{Conv}\left(V^{1}\right)$; hence, $v_{2}=\underline{v}^{1}$. Next, for agents $k \in\{2, \ldots, i\}$, in increasing order, consider profile $V^{k}=\left(V_{-k}^{k-1}, \bar{V}_{k}\right)$. By the arguments presented for $V^{1}, \bar{F}\left(V^{k}\right)=\bar{F}(V)$. Therefore, at profile $V^{i}=\left(\bar{V}_{1}, \ldots, \bar{V}_{i}, V_{i+1}, \ldots, V_{n}\right), \bar{F}\left(V^{i}\right)=\bar{F}(V)$. Finally, begin from profile $V^{\prime}$. By the same technique as the one described for profile $V$, change the preferences of agents $k \in\{1, \ldots, i-i\}$, in increasing order, to again construct profile $V^{i}=\left(\bar{V}_{1}, \ldots, \bar{V}_{i}, V_{i+1}, \ldots, V_{n}\right)$. Therefore, $\bar{F}\left(V^{i}\right)=\bar{F}\left(V^{\prime}\right)=\bar{F}(V)$.

Similarly, if $v_{i}^{\prime} \leq \underline{F}(V)$, then once can show that $\underline{F}\left(V^{\prime}\right)=\underline{F}(V)$, by using symmetrical arguments to the ones presented above. Specifically, begin from profile $V$ and change the preferences of agents $k \in\{i, \ldots, n\}$, in decreasing order, and show that $\underline{F}\left(V_{1}, \ldots, V_{i-1}, \bar{V}_{i}, \ldots, \bar{V}_{n}\right)=\underline{F}(V)$. Finally, begin from profile $V^{\prime}$ and change the preferences of agents $k \in\{i+1, \ldots, n\}$, in decreasing order, and show that $\underline{F}\left(V_{1}, \ldots, V_{i-1}, \bar{V}_{i}, \ldots, \bar{V}_{n}\right)=\underline{F}\left(V^{\prime}\right)=\underline{F}(V)$.

\section{D Proof of Theorem 3.1 (equivalence of statements (ii) and (iii))}

We first show for Theorem 3.1 that statement (iii) implies statement (ii) in domain $\mathcal{R}$. Moreover, as discussed in Section 3.5.3, this result also holds in domain $\mathcal{S}$.

Proof of Theorem 3.1 (statement (iii) implies statement (ii)). Let $F_{G}^{\alpha, \beta} \in$ $\mathcal{F}_{G}$. By the definition of $\mathcal{F}_{G}$, to show that $F_{G}^{\alpha, \beta}$ satisfies uncompromisingness, it suffices to show that the minimum and maximum chosen alternatives by $F_{G}^{\alpha, \beta}$ are not compromised. Moreover, by symmetry of arguments, we only need to show that $\underline{F}_{G}^{\alpha, \beta}(V)$ is not compromised.

Let $V \in \mathcal{R}^{N}$ and without loss of generality, let $v_{1} \leq \cdots \leq v_{n}$. Let $i \in N$ and $V^{\prime} \in \mathcal{R}^{N}$ be such that $V_{-i}=V_{-i}^{\prime}$. Moreover, let $v_{i} \neq \underline{F}_{G}^{\alpha, \beta}(V)$. Hence, $\underline{F}_{G}^{\alpha, \beta}(V)=\operatorname{med}\left(v, \tilde{\alpha}_{v}\right)$ and $\underline{F}_{G}^{\alpha, \beta}\left(V^{\prime}\right)=\operatorname{med}\left(v^{\prime}, \tilde{\alpha}_{v^{\prime}}\right)$. There are two cases. 
Case 1. Let $j \in N$ and $\underline{F}_{G}^{\alpha, \beta}(V)=\operatorname{med}\left(v, \tilde{\alpha}_{v}\right)=v_{j}$. Hence, $v_{i} \neq \underline{F}_{G}^{\alpha, \beta}(V)$ implies $i \neq j$. Since at $V, v_{1} \leq \cdots \leq v_{n}$, at least $j$ agents announce peaks smaller than or equal to $v_{j}$ and at least $n-j+1$ agents announce peaks larger than or equal to $v_{j}$. Thus, since there are $n$ agents in total and $\tilde{\alpha}_{v} \in A^{n+1}$, by the median operator, vector $\tilde{\alpha}_{v}$ contains at least $n-j+1$ coordinates smaller than or equal to $v_{j}$ and at least $j$ coordinates larger than or equal to $v_{j}$. Therefore, since $\alpha_{N} \leq \cdots \leq \alpha_{\emptyset}$, if $j=1, \alpha_{\{1\}} \leq v_{j} \leq \alpha_{\emptyset}$, and otherwise, $\alpha_{\{1, \ldots, j\}} \leq v_{j} \leq \alpha_{\{1, \ldots, j-1\}}$. There are two sub-cases.

(i) Let $v_{i}<\underline{F}_{G}^{\alpha, \beta}(V)=v_{j}$, that is, $i \in\{1, \ldots, j-1\}$. This implies $j \in$ $\{2, \ldots, n\}$ and $\alpha_{\{1, \ldots, j\}} \leq v_{j} \leq \alpha_{\{1, \ldots, j-1\}}$. In addition, let $v_{i}^{\prime} \leq v_{j}$. Thus, at profile $V^{\prime}$, at least $j$ agents announce peaks smaller than or equal to $v_{j}$ (i.e., agents $1, \ldots, j)$ and at least $n-j+1$ agents announce peaks larger than or equal to $v_{j}$ (i.e., agents $j, \ldots, n$ ). Moreover, $V_{-i}=V_{-i}^{\prime}$ and $v_{i}^{\prime} \leq v_{j}$ imply that $v_{i}^{\prime} \leq v_{j} \leq v_{j+1} \leq \cdots \leq v_{n}$, that is, the agents announcing the $j-1$ smallest peaks at $V$ (i.e., agents $1, \ldots, j-1$ ) also announce the $j-1$ smallest peaks at $V^{\prime}$. Similarly, the agents announcing the $j$ smallest peaks at $V$ (i.e., agents $1, \ldots, j$ ) also announce the $j$ smallest peaks at $V^{\prime}$. Hence, coordinates $\alpha_{\{1, \ldots, j\}}$ and $\alpha_{\{1, \ldots, j-1\}}$ are included in vector $\tilde{\alpha}_{v^{\prime}}$. Thus, $\alpha_{\{1, \ldots, j\}} \leq v_{j} \leq \alpha_{\{1, \ldots, j-1\}}$ and the definition of $\mathcal{F}_{G}$ implies that vector $\tilde{\alpha}_{v^{\prime}}$ contains at least $n-j+1$ coordinates smaller than or equal to $v_{j}$ and at least $j$ coordinates larger than or equal to $v_{j}$. Therefore, $\underline{F}_{G}^{\alpha, \beta}\left(V^{\prime}\right)=\operatorname{med}\left(v^{\prime}, \tilde{\alpha}_{v^{\prime}}\right)=v_{j}=\underline{F}_{G}^{\alpha, \beta}(V)$.

(ii) Let $v_{i}>\underline{F}_{G}^{\alpha, \beta}(V)=v_{j}$, that is, $i \in\{j+1, \ldots, n\}$. The proof is symmetric to (i).

Case 2. Let $M \subseteq N$ such that $|M|=m$. Let $\underline{F}_{G}^{\alpha, \beta}(V)=\operatorname{med}\left(v, \tilde{\alpha}_{v}\right)=\alpha_{M}$, such that for each $i \in N, v_{i} \neq \alpha_{M}$. Hence, if $|M|=0, \alpha_{M}=\alpha_{\emptyset}$, and otherwise, $\alpha_{M}=$ $\alpha_{\{1, \ldots, m\}}$. Since $\alpha_{N} \leq \cdots \leq \alpha_{\emptyset}$, vector $\tilde{\alpha}_{v}$ contains at least $n-m+1$ coordinates smaller than or equal to $\alpha_{M}$ (i.e., coordinates $\left.\alpha_{\{1, \ldots, m\}}, \ldots, \alpha_{N}\right)$ and at least $m+1$ coordinates larger than or equal to $\alpha_{M}$ (i.e., coordinates $\alpha_{\emptyset}, \ldots, \alpha_{\{1, \ldots, m\}}$ ). Thus, since there are $n$ agents in total and none of their announced peaks equals $\alpha_{M}$, by the median operator, at $V, m$ agents announce peaks smaller than $\alpha_{M}$ (i.e., agents $1, \ldots, m)$ and $n-m$ agents announce peaks larger than $\alpha_{M}$ (i.e., agents $m+1, \ldots, n)$. Therefore, since $v_{1} \leq \cdots \leq v_{n}$, if $m=0, \alpha_{M}=\alpha_{\emptyset}<v_{1}$, if $m=n$, $\alpha_{M}=\alpha_{N}>v_{n}$, and otherwise, $v_{m}<\alpha_{M}=\alpha_{\{1, \ldots, m\}}<v_{m+1}$. There are four sub-cases.

(i) Let $m=0$. Hence, $\alpha_{M}=\alpha_{\emptyset}<v_{1} \leq v_{i}$. In addition, let $\alpha_{M}=\underline{F}_{G}^{\alpha, \beta} \leq v_{i}^{\prime}$. Thus, at $V^{\prime}$, all $n$ agents announce peaks larger than $\alpha_{M}$. In addition, since 
$\alpha_{N} \leq \cdots \leq \alpha_{\emptyset}=\alpha_{M}$, vector $\tilde{\alpha}_{v^{\prime}}$ contains at least $n+1$ coordinates smaller than or equal to $\alpha_{M}$ (i.e., coordinates $\left.\alpha_{\emptyset}, \ldots, \alpha_{N}\right)$ and at least 1 coordinate larger than or equal to $\alpha_{M}$ (i.e., coordinate $\left.\alpha_{\emptyset}\right)$. Therefore, $\underline{F}_{G}^{\alpha, \beta}\left(V^{\prime}\right)=\operatorname{med}\left(v^{\prime}, \tilde{\alpha}_{v^{\prime}}\right)=\alpha_{M}=$ $\underline{F}_{G}^{\alpha, \beta}(V)$.

(ii) Let $m=n$. The proof is symmetric to (i).

(iii) Let $m \in\{1, \ldots, n-1\}$ and $v_{i}<\alpha_{\{1, \ldots, m\}}=\alpha_{M}$. Hence, $v_{i} \leq v_{m}<\alpha_{M}<$ $v_{m+1}$. In addition, let $v_{i}^{\prime} \leq \alpha_{M}$. Thus, at $V^{\prime}$, at least $m$ agents announce peaks smaller than or equal to $\alpha_{M}$ (i.e., agents $\left.1, \ldots, m\right)$ and $n-m$ agents announce peaks larger than $\alpha_{M}$ (i.e., agents $m+1, \ldots, n$ ). Moreover, $V_{-i}=V_{-i}^{\prime}$ and $v_{i}^{\prime} \leq \alpha_{M}$ imply that $v_{i}^{\prime} \leq \alpha_{M}<v_{m+1} \leq \cdots \leq v_{n}$, that is, the agents announcing the $m$ smallest peaks at $V$ (i.e., agents $1, \ldots, m$ ) also announce the $m$ smallest peaks at $V^{\prime}$. Hence, coordinate $\alpha_{\{1, \ldots, m\}}$ is included in vector $\tilde{\alpha}_{v^{\prime}}$. Thus, the definition of $\mathcal{F}_{G}$ implies that vector $\tilde{\alpha}_{v^{\prime}}$ contains at least $n-m+1$ coordinates smaller than or equal to $\alpha_{M}$ and at least $m+1$ coordinates larger than or equal to $\alpha_{M}$. Therefore, $\underline{F}_{G}^{\alpha, \beta}\left(V^{\prime}\right)=\operatorname{med}\left(v^{\prime}, \tilde{\alpha}_{v^{\prime}}\right)=\alpha_{M}=\underline{F}_{G}^{\alpha, \beta}(V)$.

(iv) Let $m \in\{1, \ldots, n-1\}$ and $v_{i}>\alpha_{\{1, \ldots, m\}}=\alpha_{M}$. The proof is symmetric to (iii).

Before showing for Theorem 3.1 that statement (ii) implies statement (iii), we first prove the following intermediate result that holds in both domains $\mathcal{R}$ and $\mathcal{S}$.

Lemma 3.10. Let $F \in \mathcal{F}$ satisfy strategy-proofness, peaks-onliness, and uncompromisingness. Then, for each $i \in N$ and each pair $V, V^{\prime} \in \mathcal{R}^{N}$ such that $V_{-i}^{\prime}=V_{-i}$, if $v_{i} \leq v_{i}^{\prime}$, then $\underline{F}(V) \leq \underline{F}\left(V^{\prime}\right)$ and $\bar{F}(V) \leq \bar{F}\left(V^{\prime}\right){ }^{33}$

Proof. Let $F \in \mathcal{F}$ satisfy strategy-proofness, peaks-onliness, and uncompromisingness. Let $i \in N$ and pair $V, V^{\prime} \in \mathcal{R}^{N}$ be such that $V_{-i}^{\prime}=V_{-i}$. Since by peaks-onliness, $v_{i}=v_{i}^{\prime}$ implies $F(V)=F\left(V^{\prime}\right)$, let $v_{i}<v_{i}^{\prime}$. There are three cases.

Case 1. Let $v_{i}<\underline{F}(V)$ and $v_{i}<v_{i}^{\prime}$. Concerning the maximum alternative chosen, if $v_{i}^{\prime} \leq \bar{F}(V)$, then by uncompromisingness, $\bar{F}(V)=\bar{F}\left(V^{\prime}\right)$. Let $V_{i}^{1} \in \mathcal{R}$ be such that $v_{i}^{1}=\bar{F}(V)$. Hence, by uncompromisingness, $\bar{F}\left(V_{-i}, V_{i}^{1}\right)=\bar{F}(V)$. If $v_{i}^{\prime}>\bar{F}(V)$, then assuming $\bar{F}\left(V^{\prime}\right)<\bar{F}(V)$ leads to a contradiction as follows. Begin from $V^{\prime}$ and let agent $i$ change his announcement to $V_{i}^{1}$. Since $\bar{F}\left(V^{\prime}\right)<v_{i}^{1}<v_{i}^{\prime}$, by uncompromisingness, $\bar{F}\left(V_{-i}^{\prime}, V_{i}^{1}\right)=\bar{F}\left(V^{\prime}\right)$. Thus, $\bar{F}\left(V_{-i}^{\prime}, V_{i}^{1}\right)=\bar{F}\left(V_{-i}, V_{i}^{1}\right)$ contradicts $\bar{F}\left(V^{\prime}\right)<\bar{F}(V)$. Therefore, in both cases, $\bar{F}\left(V^{\prime}\right) \geq \bar{F}(V)$.

\footnotetext{
${ }^{33}$ Notice that this result simply shows that strategy-proofness and uncompromisingness imply peak-monotonicity, a property that we refrain from introducing formally since it is only used in the "only if" part of Theorem 3.1. Loosely speaking, this property requires the following: if an agent's announced peak moves to the right (left), then the chosen set also moves to the right (left).
} 
Concerning the minimum alternative chosen, assume $R_{i}=V_{i}$. Since $v_{i}<\underline{F}(V)$ and $\bar{F}(V) \leq \bar{F}\left(V^{\prime}\right)$, if $\left.\underline{F}\left(V^{\prime}\right)\right)<\underline{F}(V)$, then single-peakedness implies $b_{R_{i}}\left(F\left(V^{\prime}\right) R_{i}\right.$ $b_{R_{i}}(F(V))$. Hence, if at profile $V$ agent $i$ deviates by announcing $V_{i}^{\prime}$, his best point improves. This contradicts strategy-proofness. Therefore, $\underline{F}\left(V^{\prime}\right) \geq \underline{F}(V)$

Case 2. Let $\underline{F}(V) \leq v_{i}<\bar{F}(V)$ and $v_{i}<v_{i}^{\prime}$. Concerning the maximum alternative chosen, by the arguments presented in Case $1, \bar{F}\left(V^{\prime}\right) \geq \bar{F}(V)$. Concerning the minimum alternative chosen, if $\underline{F}\left(V^{\prime}\right)<\underline{F}(V)$, then $\underline{F}\left(V^{\prime}\right)<v_{i}<v_{i}^{\prime}$ and uncompromisingness imply $\underline{F}\left(V^{\prime}\right)=\underline{F}\left(V_{-i}^{\prime}, V_{i}\right)=\underline{F}(V)$. Therefore, $\underline{F}\left(V^{\prime}\right) \geq \underline{F}(V)$.

Case 3. Let $\bar{F}(V) \leq v_{i}$ and $v_{i}<v_{i}^{\prime}$. Concerning the maximum alternative chosen, if $\bar{F}\left(V^{\prime}\right)<\bar{F}(V)$, then $\bar{F}(V) \leq v_{i}<v_{i}^{\prime}$ and uncompromisingness imply $\bar{F}\left(V^{\prime}\right)=\bar{F}\left(V_{-i}^{\prime}, V_{i}\right)=\bar{F}(V)$. Similarly, concerning the minimum alternative chosen, if $\underline{F}\left(V^{\prime}\right)<\underline{F}(V)$, then $\underline{F}(V) \leq v_{i}<v_{i}^{\prime}$ and uncompromisingness imply $\underline{F}\left(V^{\prime}\right)=\underline{F}\left(V_{-i}^{\prime}, V_{i}\right)=\underline{F}(V)$.

The last part of the proof of Theorem 3.1 follows. Notice that this part holds in both domains $\mathcal{R}$ and $\mathcal{S}$.

Proof of Theorem 3.1 (statement (ii) implies statement (iii)). Let $F \in \mathcal{F}$ satisfy uncompromisingness. By Proposition 3.4, F satisfies strategy-proofness and peaks-onliness. For each $i \in N$, let pair $V_{i}^{\min }, V_{i}^{\max } \in \mathcal{R}$ be such that $v_{i}^{\min }=0$ and $v_{i}^{\max }=1$. We proceed in three steps.

Step 1. We show that at each announced profile $V \in \mathcal{R}^{N}$ and for each $i \in N$, the minimum chosen alternative is the median of: (i) the announced peak of $i$ at profile $V$ (i.e., $v_{i}$ ), (ii) the minimum chosen alternative if $i$ changes his announcement to $V_{i}^{\min }$ (i.e., $\underline{F}\left(V_{-i}, V_{i}^{\min }\right)$ ), and (iii) the minimum chosen alternative if $i$ changes his announcement to $V_{i}^{\max }$ (i.e., $\underline{F}\left(V_{-i}, V_{i}^{\max }\right)$ ). By symmetry of arguments, we do not show the equivalent result for the maximum chosen alternative.

Let $i \in N$ and $V \in \mathcal{R}^{N}$. Consider profiles $V^{\min }=\left(V_{-i}, V_{i}^{\min }\right)$ and $V^{\max }=$ $\left(V_{-i}, V_{i}^{\max }\right)$. Since $V_{-i}=V_{-i}^{\min }=V_{-i}^{\max }$ and $v_{i}^{\min } \leq v_{i} \leq v_{i}^{\max }$, by Lemma 3.10, $\underline{F}\left(V^{\min }\right) \leq \underline{F}(V) \leq \underline{F}\left(V^{\max }\right)$. There are three cases.

Case 1. Let $v_{i}<\underline{F}\left(V^{\mathrm{min}}\right) \leq \underline{F}(V)$. Since $0=v_{i}^{\min } \leq v_{i}<\underline{F}(V)$, uncompromisingness implies $\underline{F}\left(V^{\mathrm{min}}\right)=\underline{F}(V)$. Therefore, $\underline{F}\left(V^{\mathrm{min}}\right)=\underline{F}(V)=$ $\operatorname{med}\left(\underline{F}\left(V^{\min }\right), v_{i}, \underline{F}\left(V^{\max }\right)\right)$.

Case 2. Let $v_{i}>\underline{F}\left(V^{\max }\right) \geq \underline{F}(V)$. Symmetric proof to Case 1 .

Case 3. Let $\underline{F}\left(V^{\min }\right) \leq v_{i} \leq \underline{F}\left(V^{\max }\right)$. Assuming $v_{i}<\underline{F}(V)$ and thus $\underline{F}\left(V^{\min }\right)<\underline{F}(V)$ results in a contradiction as follows. Since $0=v_{i}^{\min } \leq$ $v_{i}<\underline{F}(V)$, uncompromisingness implies $\underline{F}\left(V^{\mathrm{min}}\right)=\underline{F}(V)$. Similarly, assuming $\underline{F}(V)<v_{i}$ and thus $\underline{F}(V)<\underline{F}\left(V^{\max }\right)$ results in a contradiction as follows. Since 
$\underline{F}(V)<v_{i} \leq v_{i}^{\max }$, uncompromisingness implies $\underline{F}(V)=\underline{F}\left(V^{\max }\right)$. Therefore, $\underline{F}(V)=v_{i}=\operatorname{med}\left(\underline{F}\left(V^{\min }\right), v_{i}, \underline{F}\left(V^{\max }\right)\right)$.

Step 2. We construct two vectors $\alpha$ and $\beta$. In this step of the proof and in contrast to the rest of the paper, we will use a different letter to label announced profiles $(U$ instead of $V$ ). This is done in an attempt to facilitate the notation used in Step 3 of the proof that follows.

For each $M \subseteq N$, let $U^{M} \in \mathcal{R}^{N}$ be such that all agents in $M$ announce 0 as their peak and all other agents announce 1 as their peak, i.e., $u^{M}=(\underbrace{0, \ldots, 0}_{i \in M}, \underbrace{1, \ldots, 1}_{i \in N \backslash M})$. Next, let vectors $\alpha=\left(\alpha_{M}\right)_{M \subseteq N}$ and $\beta=\left(\beta_{M}\right)_{M \subseteq N}$ be such that $\alpha_{M}=\underline{F}\left(U^{M}\right)$ and $\beta_{M}=\bar{F}\left(U^{M}\right)$, hence, $\alpha_{M} \leq \beta_{M}$. Moreover, for each $L, M \subseteq N$ such that $L \subsetneq M$ notice the following. For each $i \in M \backslash L, u_{i}^{L}=1>0=u_{i}^{M}$, and for each $j \notin M \backslash L, U_{j}^{L}=U_{j}^{M}$. Begin from profile $U^{L}$ and consider that all agents $i$ (sequentially) change their announcements to $U_{i}^{M}$. Since $u_{i}^{L}>u_{i}^{M}$, by (sequentially) applying Lemma 3.10,both $\alpha_{L} \geq \alpha_{M}$ and $\beta_{L} \geq \beta_{M}$.

Step 3. We show that $F$ is a generalized median correspondence associated with vectors $\alpha$ and $\beta$ constructed in Step 2.

Let $V \in \mathcal{R}^{N}$. Without loss of generality, index the agents in $N$ such that $v_{1} \leq \cdots \leq v_{n}$. Recall vectors $\alpha, \beta$ and profiles $U^{M}$, for $M \subseteq N$, defined in Step 2. Let vectors $\tilde{\alpha}_{v}, \tilde{\beta}_{v} \in A^{n+1}$ be such that $\tilde{\alpha}_{v}=\left(\alpha_{\emptyset}, \alpha_{\{1\}}, \alpha_{\{1,2\}}, \ldots, \alpha_{N}\right)$ and $\tilde{\beta}_{v}=\left(\beta_{\emptyset}, \beta_{\{1\}}, \beta_{\{1,2\}}, \ldots, \beta_{N}\right)$.

Since the coordinates of $\tilde{\alpha}_{v}$ are such that $0 \leq \alpha_{N} \leq \cdots \leq \alpha_{\emptyset} \leq 1$ and $u^{\emptyset}=$ $(1, \ldots, 1), \underline{F}\left(U^{\emptyset}\right)=\operatorname{med}\left(u^{\emptyset}, \tilde{\alpha}_{v}\right)=\alpha_{\emptyset}$. Moreover, for each $i \in\{1, \ldots, n\}, u^{\{1, \ldots, i\}}=$ $(\underbrace{0, \ldots, 0}_{j \in\{1, \ldots, i\}}, \underbrace{1, \ldots, 1}_{j \in\{i+1, \ldots, n\}})$ implies $\underline{F}\left(U^{\{1, \ldots, i\}}\right)=\operatorname{med}\left(u^{\{1, \ldots, i\}}, \tilde{\alpha}_{v}\right)=\alpha_{\{1, \ldots, i\}}$. Similarly for $\tilde{\beta}_{v}, \bar{F}\left(U^{\emptyset}\right)=\beta_{\emptyset}$ and for each $i \in\{1, \ldots, n\}, \bar{F}\left(U^{\{1, \ldots, i\}}\right)=\beta_{\{1, \ldots, i\}}$.

Next, for each $i \in\{1, \ldots, n\}$, let $V^{i} \in \mathcal{R}^{N}$ be such that $V^{i}=$ $\left(V_{1}, \ldots, V_{i}, V_{i+1}^{\max }, \ldots, V_{n}^{\max }\right)$ and notice that $V^{n}=V$. We show that $F(V)=$ $F_{G}^{\alpha, \beta}(V)$ by induction, in two stages.

Stage 1. We show that $F\left(V^{1}\right)=F_{G}^{\alpha, \beta}\left(V^{1}\right)$.

Consider profile $V^{1}=\left(V_{1}, V_{2}^{\max }, \ldots, V_{n}^{\max }\right)$. Recall profiles $U^{\{1\}}=$ $\left(V_{1}^{\min }, V_{2}^{\max }, \ldots, V_{n}^{\max }\right)$ and $U^{\emptyset}=\left(V_{1}^{\max }, \ldots, V_{n}^{\max }\right)$. Hence, $U^{\{1\}}=\left(V_{-1}^{1}, V_{1}^{\min }\right)$ and $U^{\emptyset}=\left(V_{-1}^{1}, V_{1}^{\max }\right)$. By Step $1, \underline{F}\left(V^{1}\right)=\operatorname{med}\left(\underline{F}\left(U^{\{1\}}\right), v_{1}, \underline{F}\left(U^{\emptyset}\right)\right)$ and $\bar{F}\left(V^{1}\right)=\operatorname{med}\left(\bar{F}\left(U^{\{1\}}\right), v_{1}, \bar{F}\left(U^{\emptyset}\right)\right)$. Hence, $\underline{F}\left(V^{1}\right)=\operatorname{med}\left(\alpha_{\{1\}}, v_{1}, \alpha_{\{\emptyset\}}\right)$ and $\bar{F}\left(V^{1}\right)=\operatorname{med}\left(\beta_{\{1\}}, v_{1}, \beta_{\{\emptyset\}}\right)$. Moreover, since $\alpha_{N} \leq \cdots \leq \alpha_{\emptyset} \leq v_{2}=\cdots=v_{n}$ and $\beta_{N} \leq \cdots \leq \beta_{\emptyset} \leq v_{2}=\cdots=v_{n}, \underline{F}\left(V^{1}\right)=\operatorname{med}\left(v, \tilde{\alpha}_{v^{1}}\right)$ and $\bar{F}\left(V^{1}\right)=\operatorname{med}\left(v, \tilde{\beta}_{v^{1}}\right)$. Therefore, $F\left(V^{1}\right)=F_{G}^{\alpha, \beta}\left(V^{1}\right)$. 
Stage 2. Let $i \in\{2, \ldots, n\}$ be such that $F\left(V^{i-1}\right)=F_{G}^{\alpha, \beta}\left(V^{i-1}\right)$. We show that $F\left(V^{i}\right)=F_{G}^{\alpha, \beta}\left(V^{i}\right)$. Notice that we only show $\underline{F}\left(V^{i}\right)=\underline{F}_{G}^{\alpha, \beta}\left(V^{i}\right)$. The proof showing $\bar{F}\left(V^{i}\right)=\bar{F}_{G}^{\alpha, \beta}\left(V^{i}\right)$ is symmetric, that is, it can be obtained using the same arguments but after replacing all references to the minimum chosen alternative and $\tilde{\alpha}_{v^{i}}$ with the equivalent references to the maximum chosen alternative and $\tilde{\beta}_{v^{i}}$ respectively.

Recall that $V^{i-1}=\left(V_{1}, \ldots, V_{i-1}, V_{i}^{\max }, \ldots, V_{n}^{\max }\right)$ and $V^{i}=\left(V_{-i}^{i-1}, V_{i}\right)$. There are three cases.

Case 1. Let $v_{i}>\underline{F}\left(V^{i}\right)$. Since $V_{-i}^{i-1}=V_{-i}^{i}$ and $\underline{F}\left(V^{i}\right)<v_{i} \leq v_{i}^{\max }$, by uncompromisingness, $\underline{F}\left(V^{i}\right)=\underline{F}\left(V^{i-1}\right)=\operatorname{med}\left(v^{i-1}, \tilde{\alpha}_{v^{i}}\right)$. Thus, $V_{-i}^{i-1}=V_{-i}^{i}$ and $\operatorname{med}\left(v^{i-1}, \tilde{\alpha}_{v^{i}}\right)<v_{i} \leq v_{i}^{\max } \operatorname{implies} \underline{F}\left(V^{i}\right)=\operatorname{med}\left(v^{i}, \tilde{\alpha}_{v^{i}}\right)=\underline{F}_{G}^{\alpha, \beta}\left(V^{i}\right)$.

Case 2. Let $v_{i}<\underline{F}\left(V^{i}\right)$ and recall that $U^{\{1, \ldots, i\}}=$ $\left(V_{1}^{\min }, \ldots, V_{i}^{\min }, V_{i+1}^{\max }, \ldots, V_{n}^{\max }\right)$. Since $v_{1} \leq \cdots \leq v_{n}$ and $v_{i}<\underline{F}\left(V^{i}\right)$, for each $j \in\{1, \ldots, i\}, v_{j}^{\min } \leq v_{j}<\underline{F}\left(V^{i}\right)$; hence, by uncompromisingness, $\underline{F}\left(V_{-j}^{i}, V_{j}^{\min }\right)=\underline{F}\left(V^{i}\right)$. Therefore, beginning from profile $V^{i}$ and considering that all agents $j \in\{1, \ldots, i\}$ (sequentially) change their announcements to $V_{j}^{\text {min }}$, implies by (sequentially applying) uncompromisingness, that $\underline{F}\left(V^{i}\right)=\underline{F}\left(U^{\{1, \ldots, i\}}\right)$ where as shown above $\underline{F}\left(U^{\{1, \ldots, i\}}\right)=\alpha_{\{1, \ldots, i\}}$. Therefore, since at profile $V^{i}$, for each $j \in\{1, \ldots, i\}, v_{j}^{i}<\alpha_{\{1, \ldots, i\}}$, and for each $k \in\{i+1, \ldots, n\}, v_{k}^{i}=v_{k}^{\max }=1 \geq \alpha_{\{1, \ldots, i\}}$, by the median operator, $\underline{F}\left(V^{i}\right)=\operatorname{med}\left(v^{i}, \tilde{\alpha}_{v^{i}}\right)=\underline{F}_{G}^{\alpha, \beta}\left(V^{i}\right)$.

Case 3. Let $v_{i}=\underline{F}\left(V^{i}\right)$. Since $V_{-i}^{i-1}=V_{-i}^{i}$ and $v_{i} \leq v_{i}^{\max }$, by Lemma 3.10, $\underline{F}\left(V^{i}\right) \leq \underline{F}\left(V^{i-1}\right)$. Thus, $v_{1} \leq \cdots \leq v_{n}$ and $v_{i}=\underline{F}\left(V^{i}\right)$, imply $v_{i-1}^{i}=v_{i-1}^{i-1} \leq$ $\underline{F}\left(V^{i-1}\right)$. There are two sub-cases.

(i) Let $v_{i-1}^{i}=\underline{F}\left(V^{i-1}\right)$. Thus, $v_{i-1}^{i}=v_{i}=\underline{F}\left(V^{i}\right)=\underline{F}\left(V^{i-1}\right)$. Hence, $\underline{F}\left(V^{i-1}\right)=\underline{F}_{G}^{\alpha, \beta}\left(V^{i-1}\right)$ implies $\operatorname{med}\left(v^{i-1}, \tilde{\alpha}_{v^{i-1}}\right)=v_{i} \leq v_{i}^{\max }$. Therefore, by the median operator, $\underline{F}\left(V^{i}\right)=v_{i}=\operatorname{med}\left(v^{i}, \tilde{\alpha}_{v^{i}}\right)=\underline{F}_{G}^{\alpha, \beta}\left(V^{i}\right)$.

(ii) Let $v_{i-1}^{i}<\underline{F}\left(V^{i-1}\right)$. Recall that at profiles $U^{\{1, \ldots, i-1\}}=$ $\left(V_{1}^{\min }, \ldots, V_{i-1}^{\min }, V_{i}^{\max }, \ldots, V_{n}^{\max }\right)$ and $U^{\{1 \ldots, i\}}=\left(V_{-i}^{\{1, \ldots, i-1\}}, V_{i}^{\min }\right), \underline{F}\left(U^{\{1, \ldots, i-1\}}\right)=$ $\alpha_{\{1, \ldots, i-1\}}$ and $\underline{F}\left(U^{\{1, \ldots, i\}}\right)=\alpha_{\{1, \ldots, i\}}$. Since $v_{i}=\underline{F}\left(V^{i}\right) \leq \underline{F}\left(V^{i-1}\right)$, it follows that $v_{i} \leq \alpha_{\{1, \ldots, i-1\}}$.

Next, begin from profile $U^{\{1, \ldots, i\}}$ and consider that all agents $j \in\{1, \ldots, i\}$ (sequentially) change their announcements to $V_{j}$, i.e., the final new profile is $V^{i}=\left(V_{1}, \ldots, V_{i}, V_{i+1}^{\max }, \ldots, V_{n}^{\max }\right)$. Since $v_{j} \geq v_{j}^{\min }$, by (sequentially) applying Lemma 3.10, $\underline{F}\left(V^{i}\right) \geq \underline{F}\left(U^{\{1, \ldots, i\}}\right)=\alpha_{\{1, \ldots, i\}}$. Hence, $v_{i} \geq \alpha_{\{\overline{1}, \ldots, i\}}$ and it follows, that $\alpha_{\{1, \ldots, i\}} \leq v_{i} \leq \alpha_{\{1, \ldots, i-1\}}$. Thus, since $\alpha_{N} \leq \cdots \leq \alpha_{\emptyset}$, vector 
$\tilde{\alpha}_{v^{i}}$ contains at least $n+1-i$ coordinates not larger than $v_{i}$ (i.e., coordinates $\left.\alpha_{\{1, \ldots, i\}}, \ldots, \alpha_{N}\right)$ and at least $i$ coordinates not smaller than $v_{i}$ (i.e., coordinates $\left.\alpha_{\emptyset}, \ldots, \alpha_{\{1, \ldots, i-1\}}\right)$. In addition, since $v_{1} \leq \cdots \leq v_{n}$, at least $i$ agents announce peaks not larger than $v_{i}$ (i.e., agents $\left.1, \ldots, i\right)$ and $n-i+1$ agents announce peaks not smaller than $v_{i}$ (i.e., agents $\left.i, \ldots, n\right)$. Therefore, by the median operator, $\underline{F}\left(V^{i}\right)=\operatorname{med}\left(v^{i}, \tilde{\alpha}_{v^{i}}\right)=v_{i}=\underline{F}_{G}^{\alpha, \beta}\left(V^{i}\right)$. 


\section{Bibliography}

Achuthankutty, G. and Roy, S. On single-peaked domains and min-max rules. MPRA Paper No. 81375, 2017.

Aizerman, M. A. and Malishevski, A. V. General theory of best variants choice. IEEE Transactions on Automatic Control, 26:1030-1040, 1981.

Arribillaga, R. P. and Massó, J. Comparing generalized median voter schemes according to their manipulability. Theoretical Economics, 11:547-586, 2016.

Arrow, K. J. A difficulty in the concept of social welfare. The Journal of Political Economy, 58:328-346, 1950.

Arrow, K. J. Rational choice functions and orderings. Economica, 26:121-127, 1959.

Barberà, S. and Jackson, M. A characterization of strategy-proof social choice functions for economies with pure public goods. Social Choice and Welfare, 11: 241-252, 1994.

Barberà, S., Barrett, C., and Pattanaik, P. On some axioms for ranking sets of alternatives. Journal of Economic Theory, 33:301-308, 1984.

Barberà, S., Gül, F., and Stacchetti, E. Generalized median voter schemes and committees. Journal of Economic Theory, 61:262-289, 1993.

Barberà, S., Bossert, W., and Pattanaik, P. K. Handbook of Utility Theory, chapter 17 Ranking Sets of Objects. Springer, Boston, MA, 2004.

Border, K. and Jordan, J. Straightforward elections, unanimity and phantom voters. Review of Economic Studies, 50:153-170, 1983.

Bossert, W. and Peters, H. Single-peaked choice. Economic Theory, 41:213-230, 2009. 
Bossert, W., Pattanaik, P., and Xu, Y. Choice under complete uncertainty: axiomatic chacterizations of some decision rules. Economic Theory, 16:295-312, 2000.

Bossert, W. and Sprumont, Y. Strategy-proof preference aggregation: Possibilities and characterizations. Games and Economic Behavior, 85:109-126, 2014.

Brand, F. and Harrenstein, P. Set-rationalizable choice and self-stability. Journal of Economic Theory, 146:1721-1731, 2011.

Chernoff, H. Rational selection of decision functions. Econometrica, 22:422-443, 1954.

Ching, S. and Thomson, W. Population-monotonic solutions in public good economies with single-peaked preferences. Social Choice and Welfare, 1996. forthcoming.

Ching, S. An alternative characterization of the uniform rule. Social Choice and Welfare, 11:131-136, 1994.

Ching, S. Strategy-proofness and "median-voters". Social Choice and Welfare, 26:473-490, 1997.

Ehlers, L. Multiple public goods and lexicographic preferences: Replacement principle. Journal of Mathematical Economics, 37:1-15, 2002.

Ehlers, L. Multiple public goods, lexicographic preferences, and single-plateaued preference rules. Games and Economic Behavior, 43:1-27, 2003.

Ehlers, L. and Klaus, B. Solidarity and probabilistic target rules. Journal of Public Economic Theory, 3:167-184, 2001.

Ehlers, L., Peters, H., and Storcken, T. Strategy-proof probabilistic decision schemes for one-dimensional single-peaked preferences. Journal of Economic Theory, 105:408-434, 2002.

Fishburn, P. C. The theory of social choice. Princeton University Press, Princeton, 1973.

Gale, D. A note on revealed preference. Economica, 27:348-354, 1960.

Gibbard, A. Manipulation of voting schemes: a general result. Econometrica, 41: 587-601, 1973. 
Gordon, S. Public decisions: Solidarity and the status quo. Games and Economic Behavior, 61:225-241, 2007a.

Gordon, S. Solidarity in choosing a location on a cycle. Social Choice and Welfare, 29:125-147, 2007b.

Gordon, S. Unanimity in attribute-based preference domains. Social Choice and Welfare, 44:13-29, 2015.

Harless, P. Solidarity with respect to small changes in preferences in public good economies with single-peaked preferences. Mathematical Social Sciences, 75: 81-86, 2015a.

Harless, P. Reaching consensus: Solidarity and strategic properties in binary social choice. Social Choice and Welfare, 45:97-121, 2015b.

Harless, P. Solidarity in preference aggregation: Improving on a status quo. Games and Economic Behavior, 95:73-87, 2016.

Heo, E. J. Strategy-proof rules for two public goods: double median rules. Social Choice and Welfare, 41:895-922, 2013.

Houthakker, H. S. Revealed preference and the utility function. Economica, 17: 159-174, 1950.

Klaus, B. Target rules for public choice economies on tree networks and in euclidean spaces. Theory and Decision, 51:13-29, 2001.

Klaus, B. and Protopapas, P. Solidarity properties of choice correspondences. Cahier de Recherches Économiques du DEEP No. 16.14, 2016.

Klaus, B. and Storcken, T. Choice correspondences for public goods. Social Choice and Welfare, 19:127-154, 2002.

Manjunath, V. Efficient and strategy-proof social choice when preferences are single-dipped. International Journal of Game Theory, 43:579-597, 2014.

March, J. G. Rationality, ambiguity, and the engineering of choice. In Bell, D., Raiffa, H., and Tversky, A., editors, Decision making: descriptive, normative and prescriptive interactions, pages 33-57. Cambridge University Press, Cambridge, 1988.

March, J. G. and Simon, H. A. Organizations. Wiley, New York, 1958. 
Miyagawa, E. Locating libriaries on a street. Social Choice and Welfare, 18: 527-541, 2001.

Moulin, H. Choice functions over a finite set: a summary. Social Choice and Welfare, 2:147-160, 1985.

Moulin, H. Axioms of cooperative decision making. Cambridge University Press, Cambridge UK, 1988.

Moulin, H. On strategy-proofness and single peakedness. Public Choice, 35:437455, 1980.

Moulin, H. The pure compensation problem: Egalitarianism versus laissez-fairism. Quarterly Journal of Economics, 102:769-783, 1987.

Nash, J. F. The bargaining problem. Econometrica, 18:155-162, 1950.

Peters, H. and Wakker, P. Warp does not apply sarp for more than two commodities. Journal of Economic Theory, 62:152-160, 1994.

Peterson, M. An introduction to decision theory. Cambridge University Press, Cambridge, 2009.

Richter, M. K. Revealed preference theory. Econometrica, 41:1075-1091, 1966.

Rose, H. Consistency of preference: the two-commodity case. Review of Economic Studies, 35:124-125, 1958.

Samuelson, P. A. A note on the pure theory of consumers' behavior. Economica, 5:61-71, 1938 .

Satterthwaite, M. Strategy-proofness and arrow's condition: existence and correspondence theorems for voting procedures and social welfare functions. Journal of Economic Theory, 10:187-217, 1975.

Schwartz, T. Choice functions, "rationality" conditions, and variations on the weak axiom of revealed preferences. Journal of Economic Theory, 13:414-427, 1976.

Sen, A. K. Choice functions and revealed preference. Review of Economic Studies, 38:307-317, 1971.

Shubik, M. Game theory in the social sciences: concepts and solutions. The MIT Press, Cambridge Massachusetts, 1982. 
Thomson, W. Problems of fair division and the egalitarian solution. Journal of Economic Theory, 31:211-226, 1983a.

Thomson, W. The fair division of a fixed supply among a growing population. Mathematics of Operations Research, 8:319-326, 1983b.

Thomson, W. The replacement principle in public good economies with singlepeaked preferences. Economics Letters, 42:31-36, 1993.

Umezawa, M. The replacement principle for the provision of multiple public goods on tree networks. Social Choice and Welfare, 38:211-235, 2012.

Vohra, R. The replacement principle and tree structured preferences. Economics Letters, 63:175-180, 1999. 


\section{Valorisation}

This thesis is comprised of one essay in Choice Theory (Chapter 1) and two essays in Social Choice Theory (Chapters 1 and 2).

Choice theory studies the "thought processes" employed by individuals when making choices and searches for "patterns" in these choices. A well-studied thought process is that of rationality, considered in Chapter 1 and occurring when an individual's choices are always perfectly logical in the following sense: if solution $a$ is preferred to solution $b$, then solution $b$ might only be chosen under circumstances where solution $a$ is not feasible/available.

Although it seems that this thought process is always followed by everyone, this is not the case; consider for example an individual ordering wine, although he prefers beer, simply because he finds him/herself at a fancy French restaurant and does not wish to stand out. Therefore, rationality-related research in choice theory not only attempts to answer the question of "how does an individual's rationality affect the observed choice patterns?" but also that of "what kind of observed choice patterns indicate that the individual is rational or not?".

To this effect, Chapter 1 provides some answers to the above questions, under the scenario that the available choices are not mutually exclusive but can be combined. For instance, the individual in the previous example might prefer beer over wine, but might also prefer French cuisine and wine over French cuisine and beer. Therefore, under this new light, his/her choices seem perfectly rational.

Our results, although preliminary when considering their "real-life" applicability, pave the way for more research in the area of "multi-variate" choice, not only in theoretical disciplines but also in applied ones (behavioural economics, psychology, marketing to name but a few) that study individuals' choices. Perhaps, this could lead to models and/or theories that could better explain the rationality (or its absence) in cases where "multi-variate" choices must be made and, at a more practical level, provide tools for industries offering consumers multiple products in a bundle (for example aeroplane tickets and hotel room combinations in travel-related websites). 
Social choice theory studies the collective choices made according to individual "preferences", over decisions that must be taken collectively, when two or more individuals are concerned.

Examples are numerous and range from everyday situations, like a group of friends having to choose which film to watch at the cinema, to events that can change the course of history, like Hitler being voted Chancellor of the Weimar Republic.

Social choice theory is neither interested in the thought process of the individuals (at least in the majority of the literature), nor on how "good" or "bad" a collective choice is. Instead it attempts to answer the question of "how is the procedure followed to aggregate individual preferences, and reach a collective decision, affected when certain requirements are imposed on this very procedure?".

For example, although in parliamentary elections in most of Europe the ruling party (or coalition of parties) is always voted for by the relative majority of voters, this is not the case in the USA, where the presidents elected in 2000 and 2016 were not voted for by the relative majority of the voters. Therefore, if we were to require that "the candidate who wins the election must get the relative majority of votes", the US election system would be ruled out as a method to aggregate individual preferences.

In this spirit, Chapters 2 and 3 propose some requirements on how an aggregation method should treat individual preferences.

Chapter 2 studies the effect of imposing some solidarity requirements on the aggregation method which "guarantee" that following a change in "circumstances", all individuals will be affected in the same way; they will either all like the effect said change in circumstances will have on the collective choice or they will all dislike it. In other words, as long as one individual dislikes this effect, all others follow suit in solidarity.

To be more specific, the solidarity requirements considered are two. The first, population-monotonicity, considers changes in the population and the effect an addition (or removal) of individuals might have on the collective choice; for example, a couple that takes their kid to the cinema has to also consider his/her preferences as well. The second, replacement-dominance, considers changes in the preferences of an individual, and how these changes might affect the collective choice; for example, the same couple taking their kid to the cinema before or after he/she becomes a teenager (and hence allowed to watch many more movies).

Chapter 3 studies the effect of imposing the following two requirements on the aggregation method. 
First, ensuring that all individuals have an equal power in affecting the collective choice; in other words, everyone getting an "equal" vote on the outcome. This requirement is usually satisfied in parliamentary elections (everyone gets one vote) but not in other instances, like a shareholders' meeting (each share gets one vote, not each shareholder).

Second, that all individuals do not lie, but truthfully "announce" their preference. This requirement is never satisfied in parliamentary elections with more than two parties; if I dislike party $a$ the most, I might vote for party $b$ that has a fighting chance of winning the election, even though party $c$ is my top choice (but does not have a chance of winning).

When considering our results' applicability outside of theoretical microeconomics, there are two "take-home" messages deriving from them.

First, imposing any of the available combinations of the above described requirements, greatly limit the available aggregating methods which can be used to reach a collective choice. Moreover, since in the theoretical models considered, we assume some strong simplifications, it can be argued that said requirements, are too "demanding"; they would rule out all available aggregating methods in real-life situations -where simplifications cannot be assumed. Simply put, said requirements would be impossible to impose.

Second, an implication that is evident not just from chapters 2 and 3, but from the social choice literature in general, is the need to reconsider the way aggregating methods are chosen in all areas where a collective choice must be reached, either if this concerns how kids are assigned to schools in their district, or if this concerns how governments are elected. That is, the choice of an aggregating method to deal with a voting situation should not depend on whether the method looks attractive and is widely used, but on whether it satisfies certain requirements that are considered positive while avoiding others that are considered negative. 
